Supporting Information for:

\title{
Nickel-Catalyzed Decarbonylative Synthesis of Fluoroalkyl Thioethers
}

\author{
Conor E. Brigham, Christian A. Malapit, Naish Lalloo, and Melanie S. Sanford* \\ Department of Chemistry, University of Michigan, 930 North University Avenue, Ann Arbor, Michigan \\ 48109, United States
}

Corresponding author: *mssanfor@umich.edu

\section{Table of Contents}

$\begin{array}{ll}\text { I. General information } & \mathbf{S 2}\end{array}$

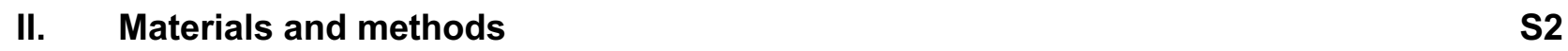

III. Procedure and spectral data for in situ generation of II-P $\mathrm{P}^{n} \mathrm{Bu}_{3} \quad \mathrm{S3}$

IV. Stoichiometric reaction of $\mathrm{Ni}(\mathrm{cod})_{2} / \mathrm{dppf}$ with $1 \mathrm{a} \quad \mathrm{S5}$

V. Ligand optimization $\quad S 6$

$\begin{array}{lll}\text { VI. } & \text { Synthesis of difluoromethyl thioesters } & \text { S8 }\end{array}$

VII. Synthesis of 1v $\quad$ S16

VIII. Synthesis of fluoroalkyl thioesters $\quad S 17$

IX. Synthesis of fluoroalkyl thioethers $\quad \mathbf{S 2 0}$

X. Catalyst screening for thiol trifluoromethylation $\quad S 31$

XI. Stoichiometric decarbonylation of trifluoromethylthioesters $\quad S 32$

XII. Heteroaromatic thiols and other failed substrates $\$$

XIII. References $\quad S 33$

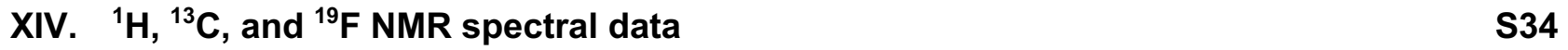




\section{General Information}

All manipulations were performed inside an $\mathrm{N}_{2}$-filled glovebox unless otherwise noted. NMR spectra were obtained on a Varian VNMR $700\left(699.76 \mathrm{MHz}\right.$ for $1 \mathrm{H} ; 175.95 \mathrm{MHz}$ for $\left.{ }^{13} \mathrm{C}\right)$, Varian VNMR $500\left(500.09 \mathrm{MHz}\right.$ for ${ }^{1} \mathrm{H}$; $470.56 \mathrm{MHz}$ for ${ }^{19} \mathrm{~F} ; 125.75 \mathrm{MHz}$ for $\left.{ }^{13} \mathrm{C}\right)$, or Varian VNMR 400 (401 MHz for ${ }^{1} \mathrm{H}$; $376 \mathrm{MHz}$ for ${ }^{19} \mathrm{~F} ; 123 \mathrm{MHz}$ for ${ }^{13} \mathrm{C}$ ) spectrometer. ${ }^{1} \mathrm{H}$ and ${ }^{13} \mathrm{C}$ NMR chemical shifts are reported in parts per million (ppm) relative to TMS, with the residual solvent peak used as an internal reference. ${ }^{19} \mathrm{~F}$ NMR chemical shifts are reported in ppm and are referenced to 4fluorotoluene $(-118.00 \mathrm{ppm})$. Abbreviations used in the NMR data are as follows: s, singlet; $d$, doublet; t, triplet; q, quartet; m, multiplet; br, broad signal. Yields of reactions that generated fluorinated products were determined by ${ }^{19} \mathrm{~F}$ NMR spectroscopic analysis using a relaxation delay of $25 \mathrm{~s}$ with a $90^{\circ}$ pulse angle. Mass spectral data were obtained on a Micromass Magnetic Sector Mass Spectrometer in electrospray ionization mode. Flash chromatography was performed using a Biotage Isolera One system with cartridges containing high performance silica gel.

\section{Materials and Methods}

All commercially available reagents were used as received unless otherwise stated. $\mathrm{Ni}(\mathrm{cod})_{2}$ (Strem) and phosphine ligands (Alfa Aesar, Acros Organics, Oakwood Scientific) were stored in a glovebox. Difluoroacetic anhydride (Oakwood Chemicals) and pyridine (Sigma) were purchased and used as received. Thiol reagents and carboxylic acids were purchased from commercial sources (Sigma, Alfa Aesar, Matrix Scientific, Frontier Scientific, Synquest, TCI America) and used as received. Deuterated solvents were purchased from Cambridge Isotope Laboratories, Inc. 


\section{Procedure and spectral data for in situ generation of II-P ${ }^{n} \mathrm{Bu}_{3}$}

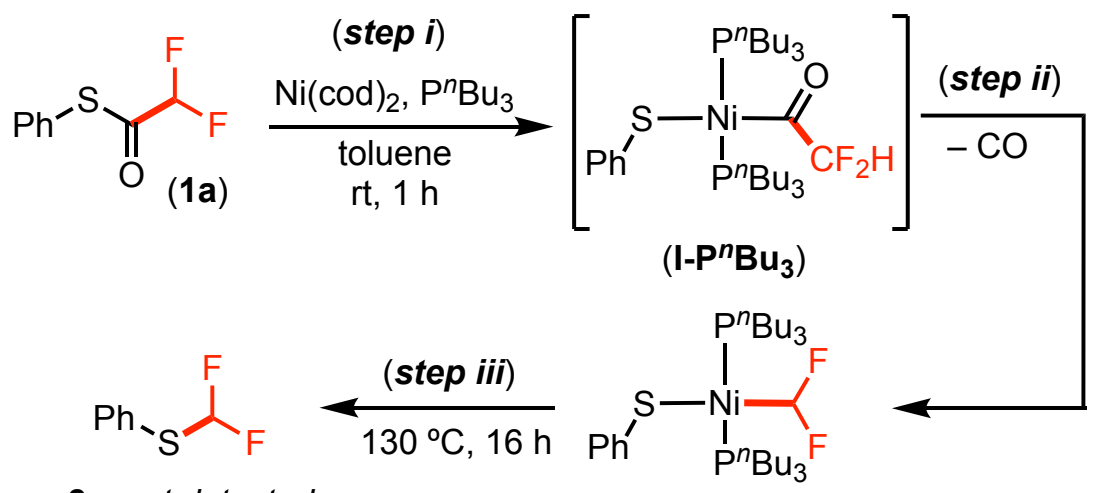

2a, not detected

$$
\text { (II-Pn } \mathrm{Bu}_{3} \text { ), 81\% }
$$

Stoichiometric reaction of $\mathrm{Ni}(\operatorname{cod})_{2} / \mathrm{P}^{n} \mathrm{Bu}_{3}$ with $1 \mathrm{a}$. $\mathrm{Ni}(\operatorname{cod})_{2}(20.6 \mathrm{mg}, 0.075 \mathrm{mmol}, 1.5$ equiv) and $\mathrm{P}^{n} \mathrm{Bu}_{3}$ (30.3 mg, $0.15 \mathrm{mmol}, 3.0$ equiv) were dissolved in $0.5 \mathrm{~mL}$ of toluene- $d_{8}$. The solution was stirred at room temperature for $15 \mathrm{~min}$, at which point it was transferred to a pre-weighed mixture of $1 \mathrm{a}$ ( $9.4 \mathrm{mg}, 0.05 \mathrm{mmol}, 1.0$ equiv) and the internal standard 4-fluorotoluene (5.5 mg, $0.05 \mathrm{mmol}, 1.0$ equiv). The reaction was allowed to stir at ambient temperature for $1 \mathrm{~h}$, at which point the solution was transferred to a screw cap NMR tube and sealed with a Teflon-lined cap. ${ }^{19} \mathrm{~F} \mathrm{NMR}$ and ${ }^{31} \mathrm{P}$ NMR spectra were then recorded (Figures $\mathrm{S} 1$ and $\mathrm{S} 2$, respectively). ${ }^{19} \mathrm{~F}$ and ${ }^{31} \mathrm{P}$ NMR spectroscopic analysis showed high conversion of the thioester starting material to intermediate II-P ${ }^{n} \mathrm{Bu}_{3}\left[{ }^{19} \mathrm{~F}\right.$ NMR: $\delta-91.49$ (dt, $\left.J=51.6,26.0 \mathrm{~Hz}\right) ;{ }^{31} \mathrm{P}$ NMR: $\delta 11.85$ (t, $J=27.0$ $\mathrm{Hz})]$. The observed three-bond $\mathrm{P}-\mathrm{F}$ coupling, is consistent with a trans ligand configuration. The temperature of the reaction was then elevated to $130{ }^{\circ} \mathrm{C}$ for $16 \mathrm{~h} .{ }^{19} \mathrm{~F}$ and ${ }^{31} \mathrm{P}$ NMR spectroscopic analysis revealed complete decomposition of II-P ${ }^{n} \mathrm{Bu}_{3}$, but the formation of $2 \mathrm{a}$ was not observed. 


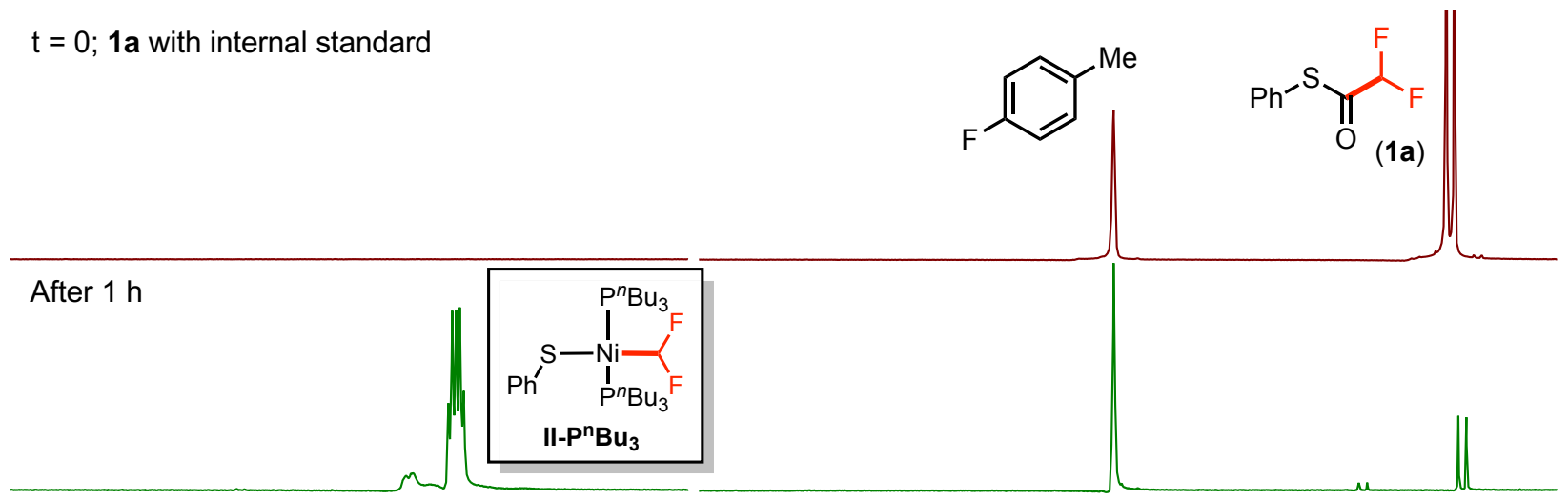

After $16 \mathrm{~h} @ 130{ }^{\circ} \mathrm{C}$

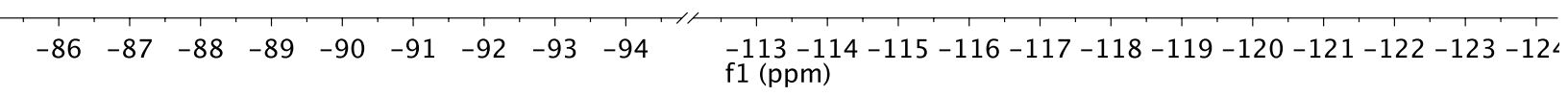

Figure S1. ${ }^{19} \mathrm{~F}$ NMR spectral data for in situ generated II-P ${ }^{n} \mathrm{Bu}_{3}$.

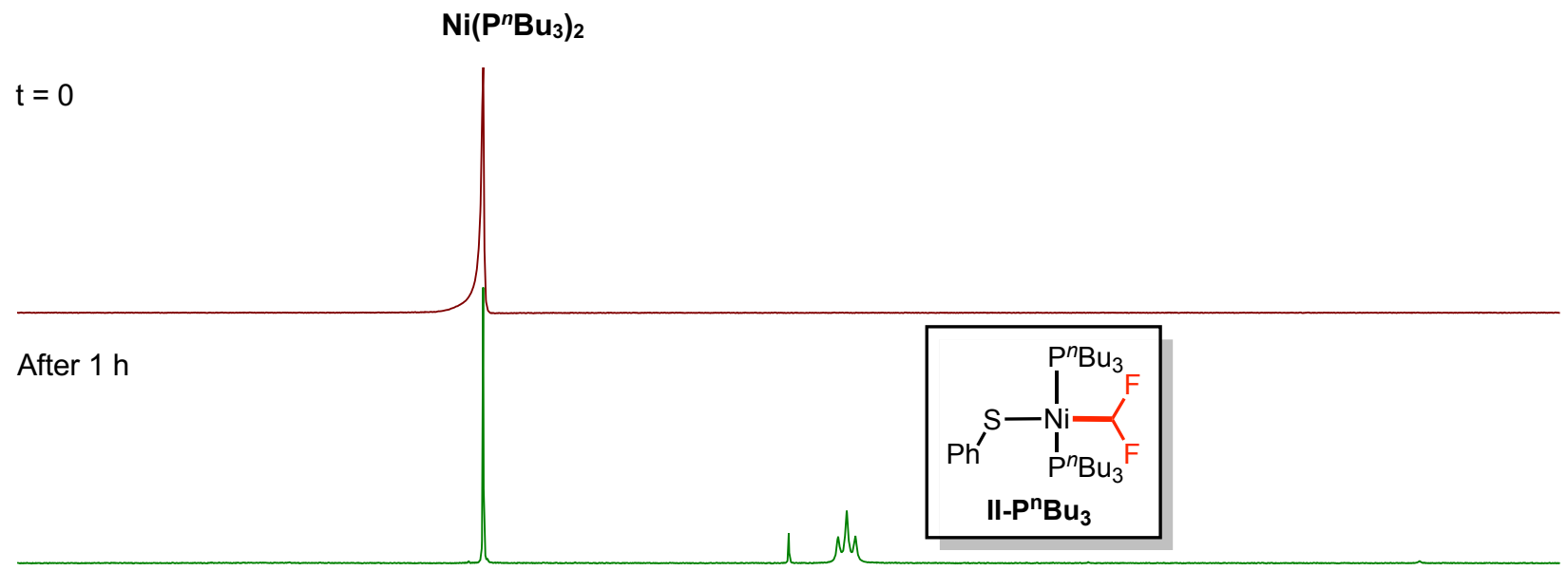

After $16 \mathrm{~h} @ 130^{\circ} \mathrm{C}$

$\begin{array}{llllllllllllllllllllllll}24 & 23 & 22 & 21 & 20 & 19 & 18 & 17 & 16 & 15 & 14 & \begin{array}{c}13 \\ \mathrm{f} 1(\mathrm{ppm})\end{array} & 11 & 10 & 9 & 8 & 7 & 6 & 5 & 4 & 3 & 2 & 1\end{array}$

Figure S2. ${ }^{31} \mathrm{P}$ NMR spectral data of in situ generated II-P ${ }^{n} \mathrm{Bu}_{3}$. 


\section{Stoichiometric reaction of $\mathrm{Ni}(\operatorname{cod})_{2} / \mathrm{dppf}$ with $1 \mathrm{a}$}

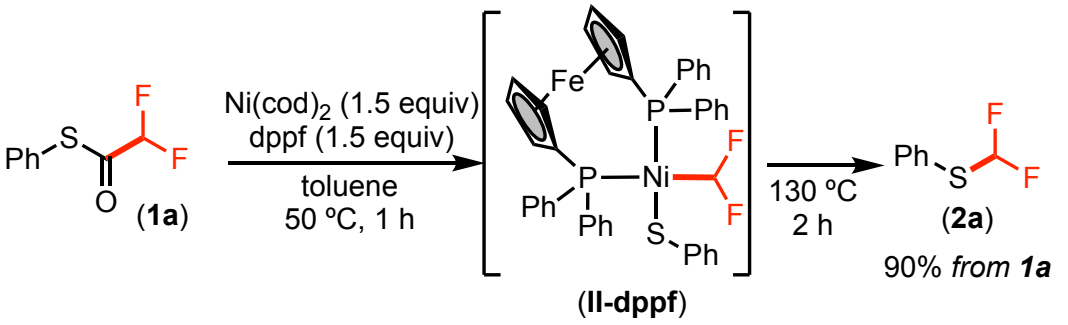

Stoichiometric reaction of $\mathrm{Ni}(\mathrm{cod})_{2} / \mathrm{dppf}$ with $1 \mathrm{a} . \mathrm{Ni}(\operatorname{cod})_{2}(20.6 \mathrm{mg}, 0.075 \mathrm{mmol}, 1.5 \mathrm{equiv})$ and dppf (30.3 mg, $0.075 \mathrm{mmol}, 1.5$ equiv) were dissolved in $0.5 \mathrm{~mL}$ of toluene. The solution was stirred at room temperature for $15 \mathrm{~min}$, at which point it was transferred to a pre-weighed mixture of $1 \mathrm{a}$ (9.4 mg, $0.05 \mathrm{mmol}, 1.0$ equiv) and the internal standard 4-fluorotoluene (0.05 mmol, 1.0 equiv). After mixing, the solution was transferred to a screw cap NMR tube and sealed with a Teflon-lined cap. The reaction mixture was heated at $50{ }^{\circ} \mathrm{C}$ for $1 \mathrm{~h}$, at which point the ${ }^{19} \mathrm{~F}$ NMR spectrum was recorded. At this time point, ${ }^{19} \mathrm{~F}$ NMR spectroscopic analysis (Figure S3) revealed some formation of the target product $\mathbf{2 a}$. Furthermore, broad signals were present in the region between -86 and -93 ppm. While these species could not be fully characterized, the broad resonances are consistent with fluxional (dppf)Ni" complexes (likely II-dppf). ${ }^{1}$ Additional heating up to $130{ }^{\circ} \mathrm{C}$ accelerated the reaction further, and all starting material was converted to $\mathbf{2 a}$ within $2 \mathrm{~h}$.

1a with internal standard
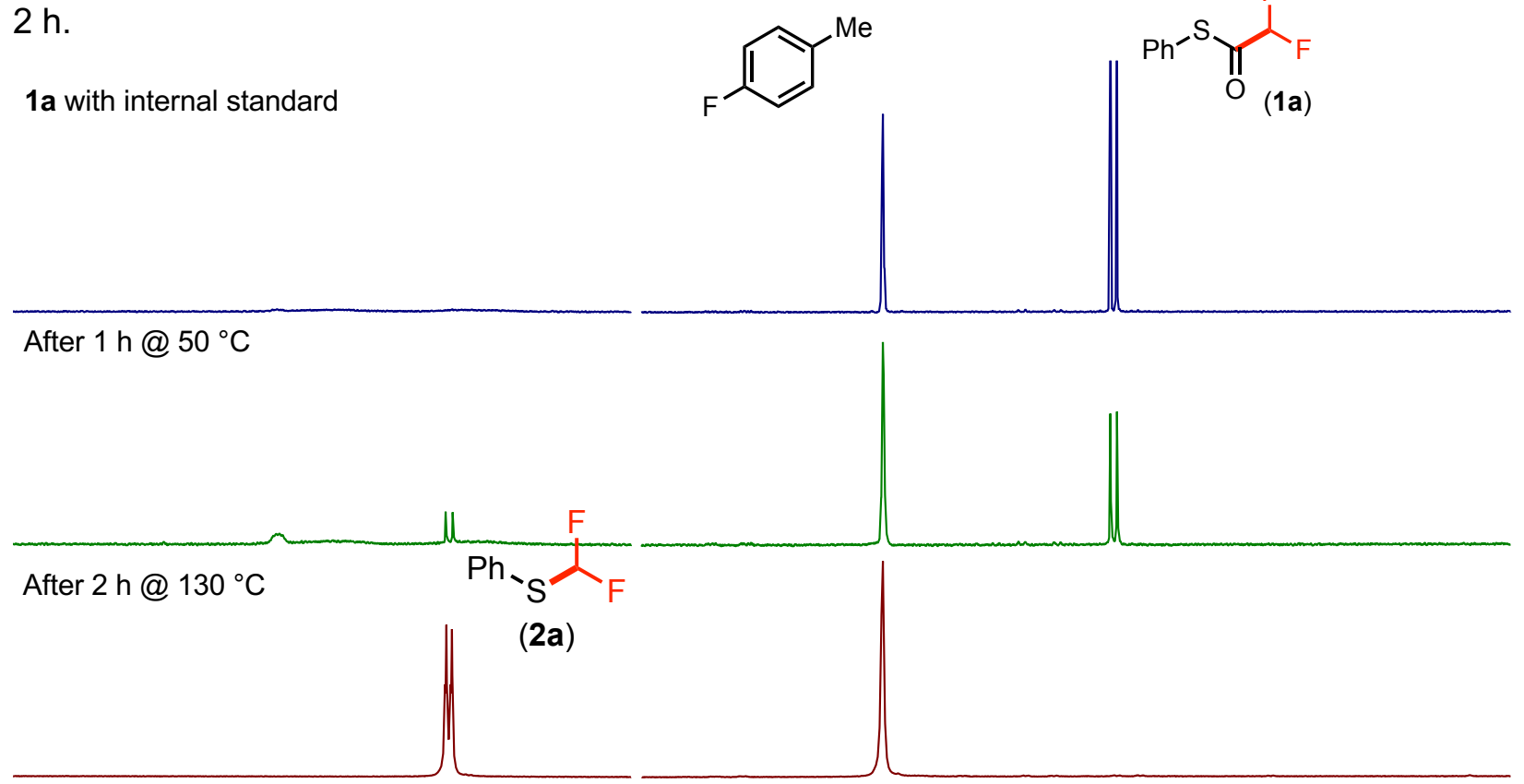

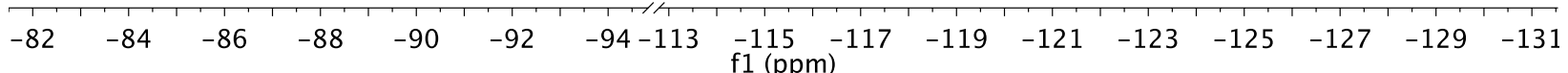

Figure S3. ${ }^{19} \mathrm{~F}$ NMR spectral data for stoichiometric reaction between Ni/dppf and 1a. 


\section{Ligand optimization}

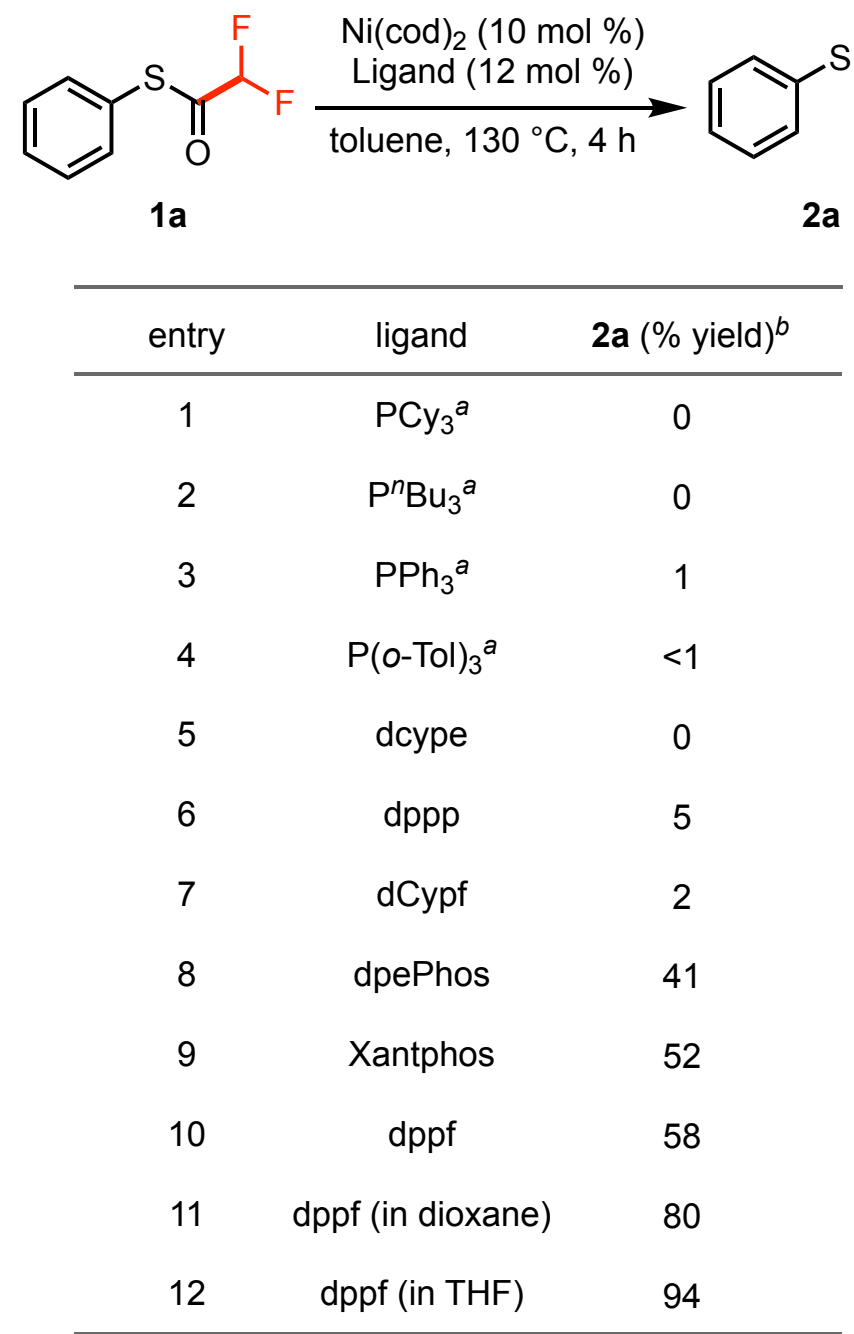

${ }^{a} 24 \mathrm{~mol} \%$ used, ${ }^{b}$ Yields determined by ${ }^{19} \mathrm{~F}$ NMR

General procedure for optimizing the catalytic decarbonylation of difluoromethyl thiophenol ester 1a. $\mathrm{Ni}(\mathrm{cod})_{2}(4.1 \mathrm{mg}, 0.015 \mathrm{mmol}, 0.1$ equiv) and the appropriate phosphine ligand $(0.036 \mathrm{mmol}, 0.24$ equiv for monodentate ligands; $0.018 \mathrm{mmol}, 0.12$ equiv for bidentate ligands) were dissolved in $0.2 \mathrm{~mL}$ of solvent. The solution was stirred at room temperature for 15 min in a tall $10 \mathrm{~mL}$ vial (Figure S4), at which point $1 \mathrm{a}(28.2 \mathrm{mg}, 0.15 \mathrm{mmol}, 1.0$ equiv) was added via syringe in $0.1 \mathrm{~mL}$ of solvent. The vial was sealed with a Teflon-lined screw cap, brought out of the glovebox, and stirred at $130^{\circ} \mathrm{C}$. After $4 \mathrm{~h}$ of heating, the reaction mixture was allowed to cool to room temperature. A stock solution of 4-fluorotoluene was prepared ( $0.3 \mathrm{M}$ in toluene) and added to the cooled reaction mixture $(0.5 \mathrm{~mL}, 0.15 \mathrm{mmol}, 1$ equiv). A sample of the crude reaction mixture with internal standard was removed for NMR analysis. Yields of $2 \mathbf{a}$ reported were determined by ${ }^{19} \mathrm{~F}$ NMR spectroscopy. 


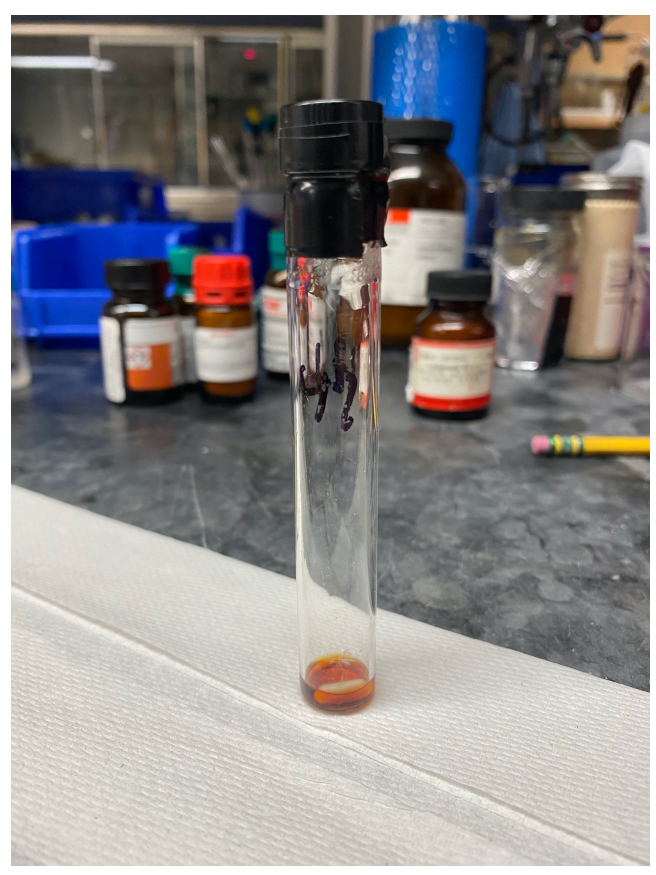

Figure S4. Reaction set-up of a $0.15 \mathrm{mmol}$ scale in a capped $10-\mathrm{mL}$ tall vial. 


\section{Synthesis of difluoromethyl thioesters}

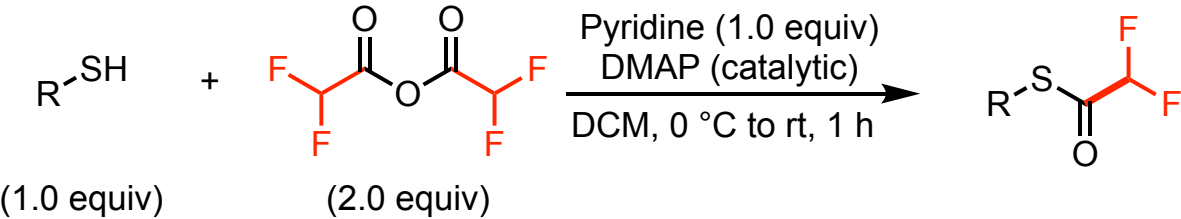

General procedure for the synthesis of difluoromethyl thioesters from difluoromethylacetic anhydride. The appropriate thiol $(3.0 \mathrm{mmol}, 1.0$ equiv) was weighed into a $20 \mathrm{~mL}$ vial and dissolved in $\mathrm{CH}_{2} \mathrm{Cl}_{2}(8 \mathrm{~mL})$. DMAP $(5.0 \mathrm{mg}, 0.04 \mathrm{mmol}$ ) was added, followed by pyridine $\left(0.24 \mathrm{~mL}, 3 \mathrm{mmol}, 1.0\right.$ equiv). The solution was then cooled to $0{ }^{\circ} \mathrm{C}$, at which point difluoroacetic anhydride (6.0 mmol, 2.0 equiv) was slowly added while stirring. The reaction was allowed to warm to room temperature and stir for $1 \mathrm{~h}$. At the end of the reaction, the solution was diluted with $\mathrm{CH}_{2} \mathrm{Cl}_{2}(30 \mathrm{~mL})$ and washed with cold water $(3 \times 10 \mathrm{~mL})$. The organic layer was collected and dried over $\mathrm{MgSO}_{4}$. Solvent was removed in vacuo to afford the difluoromethylthioester product. The difluoromethylthioester products were characterized and used in catalysis without further purification, unless stated otherwise.<smiles>O=C(Sc1ccccc1)C(F)F</smiles>

S-Phenyl 2,2-difluoroethanethioate (1a). The general method was followed using $2 \mathrm{mmol}$ of the corresponding thiol. This afforded $1 \mathrm{a}$ as a clear oil $\left(274 \mathrm{mg}, 74 \%\right.$ yield). ${ }^{1} \mathrm{H}$ NMR (500 MHz, $\left.\mathrm{CDCl}_{3}\right) \delta 7.51-7.38$ (multiple peaks, $5 \mathrm{H}$ ), $5.98(\mathrm{t}, J=54.0 \mathrm{~Hz}, 1 \mathrm{H}) \cdot{ }^{13} \mathrm{C} \mathrm{NMR}\left(126 \mathrm{MHz}, \mathrm{CDCl}_{3}\right) \delta$ $189.81(\mathrm{t}, J=29.2 \mathrm{~Hz}), 134.62,130.39,129.67,123.96,109.31(\mathrm{t}, J=255.5 \mathrm{~Hz}) .{ }^{19} \mathrm{~F}$ NMR $(377$ $\mathrm{MHz}_{\mathrm{CDCl}}$ ) $\delta-123.62$ (d, J = 54.0 Hz, 2F). HRMS (ES) calcd. for $\mathrm{C}_{8} \mathrm{H}_{6} \mathrm{~F}_{2} \mathrm{OS}[\mathrm{M}+] \mathrm{m} / \mathrm{z} 188.0107$, found 188.0103 .<smiles>COc1ccc(SC(=O)C(F)F)cc1</smiles>

S-(4-Methoxyphenyl) 2,2-difluoroethanethioate (1b). The general method was followed using $2 \mathrm{mmol}$ of the corresponding thiol. This afforded $\mathbf{1 b}$ as a clear oil (363 mg, 83\% yield). ${ }^{1} \mathrm{H} \mathrm{NMR}$ $\left(500 \mathrm{MHz} \mathrm{CDCl}_{3}\right) \delta 7.35(\mathrm{~d}, J=8.8 \mathrm{~Hz}, 2 \mathrm{H}), 6.99(\mathrm{~d}, J=8.8 \mathrm{~Hz}, 2 \mathrm{H}), 5.96(\mathrm{t}, J=54.0 \mathrm{~Hz}, 1 \mathrm{H})$, $3.84(\mathrm{~s}, 3 \mathrm{H}) .{ }^{13} \mathrm{C}$ NMR $\left(126 \mathrm{MHz} \mathrm{CDCl}_{3}\right) \delta 190.67$ (t, J=28.9 Hz), 161.37, 136.17, 115.36, 114.19, 
109.35 (t, $J=255.4 \mathrm{~Hz}), 55.39 .{ }^{19} \mathrm{~F} \mathrm{NMR}\left(377 \mathrm{MHz}, \mathrm{CDCl}_{3}\right) \delta-123.54(\mathrm{~d}, J=54.0 \mathrm{~Hz}, 2 \mathrm{~F})$. HRMS (ES) calcd. for $\mathrm{C}_{9} \mathrm{H}_{8} \mathrm{~F}_{2} \mathrm{OS}[\mathrm{M}+] \mathrm{m} / \mathrm{z} 218.0213$, found 218.0207 .

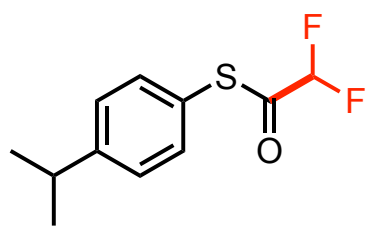

S-(4-Isopropylphenyl) 2,2-difluoroethanethioate (1c). The general method was followed using $2 \mathrm{mmol}$ of the corresponding thiol. This afforded $1 \mathrm{c}$ as a clear oil (410 $\mathrm{mg}, 89 \%$ yield). ${ }^{1} \mathrm{H}$ NMR $\left(500 \mathrm{MHz}, \mathrm{CDCl}_{3}\right.$ ) $\delta 7.42-7.28$ (multiple peaks, 4H), 5.97 (t, $J=54.0 \mathrm{~Hz}, 1 \mathrm{H}$ ), 2.97 (hept, $J=6.9$ $\mathrm{Hz}, 1 \mathrm{H}), 1.29(\mathrm{~d}, J=6.9 \mathrm{~Hz}, 6 \mathrm{H}) .{ }^{13} \mathrm{C}$ NMR $\left(126 \mathrm{MHz}, \mathrm{CDCl}_{3}\right) \delta 190.16(\mathrm{t}, J=29.0 \mathrm{~Hz}), 151.52$, 134.53, 127.90, 120.59 (d, $J=1.7 \mathrm{~Hz}), 109.33$ (t, $J=255.5 \mathrm{~Hz}), 34.00,23.73 .{ }^{19} \mathrm{~F}$ NMR $(471 \mathrm{MHz}$, $\left.\mathrm{CDCl}_{3}\right) \delta-123.48\left(\mathrm{~d}, J=54.0 \mathrm{~Hz}, 2 \mathrm{~F}\right.$ ). HRMS (ES) calcd. for $\mathrm{C}_{11} \mathrm{H}_{12} \mathrm{~F}_{2} \mathrm{OS}[\mathrm{M}+] \mathrm{m} / \mathrm{z} 230.0577$, found 230.0570 .<smiles>CC(C)(C)c1ccc(SC(=O)C(F)F)cc1</smiles>

S-(4-(Tert-butyl)phenyl) 2,2-difluoroethanethioate (1d). The general method was followed using $2 \mathrm{mmol}$ of the corresponding thiol. This afforded $\mathbf{1 d}$ as a clear oil $\left(350 \mathrm{mg}, 76 \%\right.$ yield). ${ }^{1} \mathrm{H}$ NMR $\left(500 \mathrm{MHz}, \mathrm{CDCl}_{3}\right) \delta 7.49(\mathrm{~d}, J=8.0 \mathrm{~Hz}, 2 \mathrm{H}), 7.38(\mathrm{~d}, J=8.3 \mathrm{~Hz}, 2 \mathrm{H}), 5.96(\mathrm{t}, J=54.0 \mathrm{~Hz}$, $1 \mathrm{H}), 1.35$ (s, 9H). ${ }^{13} \mathrm{C}$ NMR $\left(126 \mathrm{MHz}, \mathrm{CDCl}_{3}\right) \delta 190.15$ (t, $\left.J=29.0 \mathrm{~Hz}\right), 153.79,134.22,126.82$, 120.35, $109.32(\mathrm{t}, J=255.6 \mathrm{~Hz}), 34.88,31.15 .{ }^{19} \mathrm{~F} \mathrm{NMR}\left(377 \mathrm{MHz}, \mathrm{CDCl}_{3}\right) \delta-123.74(\mathrm{~d}, J=54.0$ $\mathrm{Hz}, 2 \mathrm{~F}$ ). HRMS (ES) calcd. for $\mathrm{C}_{12} \mathrm{H}_{14} \mathrm{~F}_{2} \mathrm{OS}[\mathrm{M}+] \mathrm{m} / \mathrm{z} 244.0733$, found 244.0725 .<smiles>CN(C)c1ccc(SC(=O)C(F)F)cc1</smiles>

S-(4-(Dimethylamino)phenyl) 2,2-difluoroethanethioate (1e). The general method was followed using $2 \mathrm{mmol}$ of the corresponding thiol. This afforded a crude yellow oil. 1e was purified via flash chromatography (hexanes/EtOAc, 80:20) to afford a light yellow solid (313 mg, 68\% yield). mp 43.8-46.9 ${ }^{\circ} \mathrm{C} .{ }^{1} \mathrm{H}$ NMR $\left(500 \mathrm{MHz}, \mathrm{CDCl}_{3}\right) \delta 7.25$ (d, J = 7.6 Hz, 2H), 6.74 (d, J = 7.5 $\mathrm{Hz}, 2 \mathrm{H}), 5.94(\mathrm{t}, J=54.1 \mathrm{~Hz}, 1 \mathrm{H}), 3.01(\mathrm{~s}, 6 \mathrm{H}) .{ }^{13} \mathrm{C} \mathrm{NMR}\left(126 \mathrm{MHz}, \mathrm{CDCl}_{3}\right) \delta 191.60(\mathrm{t}, J=28.5$ 
$\mathrm{Hz}), 151.56,135.69,112.82,109.46(\mathrm{t}, J=255.4 \mathrm{~Hz}), 107.57,40.10 .{ }^{19} \mathrm{~F} \mathrm{NMR}\left(376 \mathrm{MHz}, \mathrm{CDCl}_{3}\right)$ $\delta-123.54(\mathrm{~d}, J=54.1 \mathrm{~Hz}, 2 \mathrm{~F})$. HRMS (ES) calcd. for $\mathrm{C}_{12} \mathrm{H}_{14} \mathrm{~F}_{2} \mathrm{OS}[\mathrm{M}+\mathrm{H}] \mathrm{m} / \mathrm{z} 232.0629$, found 232.0611 .<smiles>O=C(Sc1ccc2ccccc2c1)C(F)F</smiles>

S-(Naphthalen-2-yl) 2,2-difluoroethanethioate (1f). The general method was followed using 3 mmol of the corresponding thiol. This afforded $1 \mathrm{f}$ as a white solid (559 mg, 78\% yield). mp 50.7$52.1^{\circ} \mathrm{C} .{ }^{1} \mathrm{H} \mathrm{NMR}\left(500 \mathrm{MHz}, \mathrm{CDCl}_{3}\right) \delta 8.02(\mathrm{~s}, 1 \mathrm{H}), 7.94(\mathrm{~d}, J=8.6 \mathrm{~Hz}, 1 \mathrm{H}), 7.90(\mathrm{~d}, J=7.9 \mathrm{~Hz}$, $1 \mathrm{H}$ ), 7.87 (d, $J=7.8 \mathrm{~Hz}, 1 \mathrm{H}$ ), 7.63-7.52 (multiple peaks, $2 \mathrm{H}$ ), 7.47 (dt, $J=8.6,1.4 \mathrm{~Hz}, 1 \mathrm{H}$ ), 6.02 $(\mathrm{t}, J=54.0 \mathrm{~Hz}, 1 \mathrm{H}) .{ }^{13} \mathrm{C}$ NMR $\left(126 \mathrm{MHz}, \mathrm{CDCl}_{3}\right) \delta 190.02(\mathrm{t}, J=29.4 \mathrm{~Hz}), 134.99,133.71,133.59$, 130.36, 129.41, 128.02, 127.89, 127.73, 126.93, 121.12, $109.34(\mathrm{t}, J=255.7 \mathrm{~Hz}) .{ }^{19} \mathrm{~F}$ NMR $(376$ $\left.\mathrm{MHz}, \mathrm{CDCl}_{3}\right) \delta-123.39\left(\mathrm{~d}, \mathrm{~J}=54.0 \mathrm{~Hz}, 2 \mathrm{~F}\right.$ ). HRMS (ES) calcd. for $\mathrm{C}_{12} \mathrm{H}_{8} \mathrm{~F}_{2} \mathrm{OS}[\mathrm{M}+] \mathrm{m} / \mathrm{z} 238.0264$, found 238.0260 .<smiles>O=C(Sc1ccc(F)cc1)C(F)F</smiles>

S-(4-fluorophenyl) 2,2-difluoroethanethioate (1g). The general method was followed using 2 mmol of the corresponding thiol. This afforded $1 \mathrm{~g}$ as a clear oil (365 mg, 88\% yield). ${ }^{1} \mathrm{H}$ NMR (500 $\left.\mathrm{MHz}_{\mathrm{CDCl}}\right) \delta 7.47-7.41(\mathrm{~m}, 2 \mathrm{H}), 7.18(\mathrm{t}, J=8.6 \mathrm{~Hz}, 2 \mathrm{H}), 5.98(\mathrm{t}, J=53.9 \mathrm{~Hz}, 1 \mathrm{H}) .{ }^{13} \mathrm{C} \mathrm{NMR}$ $\left(126 \mathrm{MHz}, \mathrm{CDCl}_{3}\right) \delta 189.88$ (t, $\left.J=29.3 \mathrm{~Hz}\right), 164.05$ (d, $\left.J=251.7 \mathrm{~Hz}\right), 136.77$ (d, J = 8.7 Hz), 119.17, 117.06 (d, $J=22.4 \mathrm{~Hz}), 109.23$ (t, $J=255.7 \mathrm{~Hz}) .{ }^{19} \mathrm{~F}$ NMR $\left(471 \mathrm{MHz}, \mathrm{CDCl}_{3}\right) \delta-109.38$ (ddd, $J=13.8,8.6,5.2 \mathrm{~Hz}),-123.54\left(\mathrm{~d}, J=54.1 \mathrm{~Hz}\right.$ ). HRMS (GC-APCI) calcd. for $\mathrm{C}_{8} \mathrm{H}_{5} \mathrm{~F}_{3} \mathrm{OS}[\mathrm{M}+]$ $\mathrm{m} / \mathrm{z} 206.0013$, found 206.0008<smiles>COC(=O)c1ccc(SC(=O)C(F)F)cc1</smiles>

methyl 4-((2,2-difluoroacetyl)thio)benzoate (1h). The general method was followed using 2 $\mathrm{mmol}$ of the corresponding thiol. This afforded $1 \mathrm{~h}$ as a white solid (444 $\mathrm{mg}, 90 \%$ yield). ${ }^{1} \mathrm{H} \mathrm{NMR}$ $\left(500 \mathrm{MHz} \mathrm{CDCl}_{3}\right) \delta 8.13(\mathrm{~d}, J=8.5 \mathrm{~Hz}, 1 \mathrm{H}), 7.55(\mathrm{~d}, J=8.4 \mathrm{~Hz}, 1 \mathrm{H}), 5.99(\mathrm{t}, J=53.9 \mathrm{~Hz}, 1 \mathrm{H})$, $3.96(\mathrm{~s}, 3 \mathrm{H}) .{ }^{13} \mathrm{C}$ NMR $\left(176 \mathrm{MHz}, \mathrm{CDCl}_{3}\right) \delta 188.85$ (t, J=29.7 Hz), 166.11, 134.36, 131.85, 130.53, 
129.51, $109.15(\mathrm{t}, J=256.0 \mathrm{~Hz}), 52.45 .{ }^{19} \mathrm{~F} \mathrm{NMR}\left(376 \mathrm{MHz}, \mathrm{CDCl}_{3}\right) \delta-123.46(\mathrm{~d}, J=54.0 \mathrm{~Hz})$. HRMS (GC-APCl) calcd. for $\mathrm{C}_{8} \mathrm{H}_{5} \mathrm{~F}_{3} \mathrm{OS}[\mathrm{M}+\mathrm{H}]+\mathrm{m} / \mathrm{z} 247.0262$, found 247.0241 .<smiles>CN(C(=O)C(F)(F)F)c1ccc(SC(=O)C(F)F)cc1</smiles>

S-(4-(N-(2,2-Difluoroacetyl)acetamido)phenyl) 2,2-difluoroethanethioate (1i). The general method was followed using $2 \mathrm{mmol}$ of the corresponding thiol. This afforded $1 \mathbf{i}$ as a clear oil (568 mg, 88\% yield). mp 101.3-103.3 ${ }^{\circ} \mathrm{C} .{ }^{1} \mathrm{H}$ NMR (500 MHz, $\left.\mathrm{CDCl}_{3}\right) \delta 7.63$ (d, J = 7.9 Hz, 2H), 7.33 $(\mathrm{d}, J=8.0 \mathrm{~Hz}, 2 \mathrm{H}), 6.65(\mathrm{t}, J=53.2 \mathrm{~Hz}, 1 \mathrm{H}), 6.01(\mathrm{t}, J=54.0 \mathrm{~Hz}, 1 \mathrm{H}), 2.19(\mathrm{~s}, 3 \mathrm{H}) .{ }^{13} \mathrm{C} \mathrm{NMR}(126$ $\left.\mathrm{MHz} \mathrm{CDCl}_{3}\right) \delta 188.75(\mathrm{t}, J=29.8 \mathrm{~Hz}), 171.97,164.84(\mathrm{t}, J=27.9 \mathrm{~Hz}), 138.41,136.10,129.84$, 126.57, 109.12 (t, J = 255.9 Hz), 107.12 (t, $J=247.6 \mathrm{~Hz}), 25.74 .{ }^{19} \mathrm{~F} \mathrm{NMR}\left(377 \mathrm{MHz}, \mathrm{CDCl}_{3}\right) \delta-$ $123.56(\mathrm{~d}, J=54.0 \mathrm{~Hz}),-125.58(\mathrm{~d}, J=53.2 \mathrm{~Hz})$. HRMS (ESI) calcd. for $\mathrm{C}_{12} \mathrm{H}_{9} \mathrm{~F}_{4} \mathrm{NO}_{3} \mathrm{~S}[\mathrm{M}+\mathrm{H}] \mathrm{m} / \mathrm{z}$ 324.0339 , found 324.0312 .<smiles>O=C(Sc1ccccc1F)C(F)F</smiles>

S-(2-fluorophenyl) 2,2-difluoroethanethioate (1j). The general method was followed using 2 $\mathrm{mmol}$ of the corresponding thiol. This afforded $1 \mathbf{j}$ as a clear oil (343 $\mathrm{mg}, 83 \%$ yield). ${ }^{1} \mathrm{H}$ NMR (500 $\left.\mathrm{MHz} \mathrm{CDCl}_{3}\right) \delta \delta 7.53$ (dddd, $\left.J=8.0,7.2,5.0,1.5 \mathrm{~Hz}, 1 \mathrm{H}\right), 7.46$ (ddd, $J=8.1,6.9,1.7 \mathrm{~Hz}, 1 \mathrm{H}$ ), $7.31-7.21(\mathrm{~m}, 2 \mathrm{H}), 6.00(\mathrm{t}, J=53.9 \mathrm{~Hz}, 1 \mathrm{H}) .{ }^{13} \mathrm{C}$ NMR $\left(126 \mathrm{MHz}, \mathrm{CDCl}_{3}\right) \delta 188.03(\mathrm{t}, J=29.7$ $\mathrm{Hz}), 163.23,161.23,136.49,133.15(\mathrm{~d}, J=8.0 \mathrm{~Hz}), 125.09(\mathrm{~d}, J=4.0 \mathrm{~Hz}), 116.61$ (d, J = 22.2 $\mathrm{Hz}), 109.13(\mathrm{t}, J=255.7 \mathrm{~Hz}) .{ }^{19} \mathrm{~F}$ NMR $\left(471 \mathrm{MHz}, \mathrm{CDCl}_{3}\right) \delta-105.79--106.12(\mathrm{~m}, 1 \mathrm{~F}),-123.43$ (dd, $J=53.9,19.1 \mathrm{~Hz}, 2 \mathrm{~F}$ ). HRMS (GC-APCl) calcd. for $\mathrm{C}_{8} \mathrm{H}_{5} \mathrm{~F}_{3} \mathrm{OS}[\mathrm{M}+] \mathrm{m} / \mathrm{z} 206.0013$, found 206.0007.<smiles>O=C(Sc1ccc(C(F)(F)F)cc1)C(F)F</smiles>

S-(4-(trifluoromethyl)phenyl) 2,2-difluoroethanethioate (1k). The general method was followed using $2 \mathrm{mmol}$ of the corresponding thiol. This afforded $1 \mathbf{k}$ as a clear oil $(425 \mathrm{mg}, 83 \%$ yield). ${ }^{1} \mathrm{H}$ NMR $\left(400 \mathrm{MHz}, \mathrm{CDCl}_{3}\right) 7.71(\mathrm{~d}, J=8.1 \mathrm{~Hz}, 1 \mathrm{H}), 7.57(\mathrm{~d}, J=8.0 \mathrm{~Hz}, 1 \mathrm{H}), 5.97$ (t, $J=$ $53.9 \mathrm{~Hz}, 1 \mathrm{H}) .{ }^{13} \mathrm{C}$ NMR $\left(176 \mathrm{MHz}, \mathrm{CDCl}_{3}\right) \delta 188.82$ (t, $\left.J=29.8 \mathrm{~Hz}\right), 134.85,132.41$ (q, $J=33.0$ 
$\mathrm{Hz}), 128.67,126.45(\mathrm{q}, J=3.8 \mathrm{~Hz}), 123.55(\mathrm{q}, J=272.7 \mathrm{~Hz}), 109.12(\mathrm{t}, J=256.0 \mathrm{~Hz}) .{ }^{19} \mathrm{~F} \mathrm{NMR}$ $\left(376 \mathrm{MHz}, \mathrm{CDCl}_{3}\right) \delta-63.10,-123.48\left(\mathrm{~d}, J=54.3 \mathrm{~Hz}\right.$ ). HRMS (GC-APCl) calcd. for $\mathrm{C}_{9} \mathrm{H}_{5} \mathrm{~F}_{5} \mathrm{OS}[\mathrm{M}+]$ $\mathrm{m} / \mathrm{z} 255.9981$, found 255.9983 .<smiles>O=C(Sc1ccccc1C(F)(F)F)C(F)F</smiles>

S-(2-(trifluoromethyl)phenyl) 2,2-difluoroethanethioate (1I). The general method was followed using $2 \mathrm{mmol}$ of the corresponding thiol. This afforded $1 \mathrm{l}$ as a clear oil $\left(462 \mathrm{mg}, 90 \%\right.$ yield). ${ }^{1} \mathrm{H}$ NMR (500 MHz, $\left.\mathrm{CDCl}_{3}\right) \delta 7.87(\mathrm{~d}, J=7.1 \mathrm{~Hz}, 1 \mathrm{H}$ ), $7.73-7.58$ (multiple peaks, $3 \mathrm{H}$ ), 6.00 (t, $J=$ $53.9 \mathrm{~Hz}, 1 \mathrm{H}) .{ }^{13} \mathrm{C}$ NMR $\left(126 \mathrm{MHz}, \mathrm{CDCl}_{3}\right) \delta 188.70(\mathrm{t}, J=29.9 \mathrm{~Hz}), 138.91,133.66(\mathrm{q}, J=30.7$ $\mathrm{Hz}), 132.68,130.91,127.61$ (q, $J=5.4 \mathrm{~Hz}$ ), 122.90 (q, $J=273.7 \mathrm{~Hz}$ ), 122.61, 109.09 (t, $J=255.7$ $\mathrm{Hz}$ ). ${ }^{19} \mathrm{~F}$ NMR (471 MHz, CDCl 3 ) $\delta-60.65$ (s, 3F), -123.45 (d, $J=53.3 \mathrm{~Hz}, 2 \mathrm{~F}$ ). HRMS (GC-APCl) calcd. for $\mathrm{C}_{9} \mathrm{H}_{5} \mathrm{~F}_{5} \mathrm{OS}[\mathrm{M}+] \mathrm{m} / \mathrm{z} 255.9981$, found 255.9980 .<smiles>Cc1ccccc1SC(=O)C(F)F</smiles>

S-(o-Tolyl) 2,2-difluoroethanethioate $(1 \mathrm{~m})$. The general method was followed using $3 \mathrm{mmol}$ of the corresponding thiol. This afforded $1 \mathrm{~m}$ as a clear oil ( $466 \mathrm{mg}, 89 \%$ yield). ${ }^{1} \mathrm{H}$ NMR (401 MHz, $\left.\mathrm{CDCl}_{3}\right) \delta 7.45-7.31(\mathrm{~m}, 3 \mathrm{H}), 7.25(\mathrm{~d}, J=3.6 \mathrm{~Hz}, 1 \mathrm{H}), 5.95(\mathrm{t}, J=53.7 \mathrm{~Hz}, 1 \mathrm{H}), 2.34(\mathrm{~s}, 3 \mathrm{H}) .{ }^{13} \mathrm{C}$ $\operatorname{NMR}\left(176 \mathrm{MHz}, \mathrm{CDCl}_{3}\right) \delta 189.26(\mathrm{t}, J=29.2 \mathrm{~Hz}), 142.43,135.89,131.18,131.00,127.03,123.41$, $109.33(\mathrm{t}, J=255.5 \mathrm{~Hz}), 20.46 .{ }^{19} \mathrm{~F} \mathrm{NMR}\left(471 \mathrm{MHz}, \mathrm{CDCl}_{3}\right) \delta-123.35(\mathrm{~d}, J=53.7 \mathrm{~Hz}, 2 \mathrm{~F})$. HRMS (ES) calcd. for $\mathrm{C}_{9} \mathrm{H}_{8} \mathrm{~F}_{2} \mathrm{OS}[\mathrm{M}+] \mathrm{m} / \mathrm{z} 202.0264$, found 202.0257 .<smiles>CC(C)c1ccccc1SC(=O)C(F)F</smiles>

S-(2-Isopropylphenyl) 2,2-difluoroethanethioate (1n). The general method was followed using $2 \mathrm{mmol}$ of the corresponding thiol. This afforded $1 \mathrm{n}$ as a clear oil $\left(370 \mathrm{mg}, 80 \%\right.$ yield). ${ }^{1} \mathrm{H}$ NMR $\left(500 \mathrm{MHz}, \mathrm{CDCl}_{3}\right) \delta 7.52-7.42(\mathrm{~m}, 2 \mathrm{H}), 7.40(\mathrm{~d}, J=7.8 \mathrm{~Hz}, 1 \mathrm{H}), 7.26(\mathrm{~d}, J=7.5 \mathrm{~Hz}, 1 \mathrm{H}), 5.97(\mathrm{t}$, $J=54.1 \mathrm{~Hz}, 1 \mathrm{H}), 3.25$ (hept, $J=6.6 \mathrm{~Hz}, 1 \mathrm{H}), 1.22(\mathrm{~d}, J=6.9 \mathrm{~Hz}, 6 \mathrm{H}) .{ }^{13} \mathrm{C} \mathrm{NMR}\left(176 \mathrm{MHz}, \mathrm{CDCl}_{3}\right)$ $\delta 189.80(\mathrm{t}, J=29.1 \mathrm{~Hz}), 152.41,136.33,131.35,126.91,126.76,122.18,109.35$ (t, $J=255.6$ 
$\mathrm{Hz}), 31.33,23.58 .{ }^{19} \mathrm{~F} \mathrm{NMR}\left(471 \mathrm{MHz}, \mathrm{CDCl}_{3}\right) \delta-123.06(\mathrm{~d}, J=54.1 \mathrm{~Hz}, 2 \mathrm{~F})$. HRMS (ES) calcd. for $\mathrm{C}_{11} \mathrm{H}_{12} \mathrm{~F}_{2} \mathrm{OS}[\mathrm{M}+] \mathrm{m} / \mathrm{z} 230.0577$, found 230.0571 .<smiles>Cc1cc(C)c(SC(=O)C(F)F)c(C)c1</smiles>

S-Mesityl 2,2-difluoroethanethioate (10). The general method was followed using $2 \mathrm{mmol}$ of the corresponding thiol. This afforded 10 as white solid (393 mg, 85\% yield). $\mathrm{mp} 69.3-72.0^{\circ} \mathrm{C}$. ${ }^{1} \mathrm{H}$ NMR $\left(500 \mathrm{MHz}, \mathrm{CDCl}_{3}\right) \delta 7.02(\mathrm{~s}, 2 \mathrm{H}), 5.95(\mathrm{t}, J=54.1 \mathrm{~Hz}, 1 \mathrm{H}), 2.32(\mathrm{~s}, 9 \mathrm{H}) .{ }^{13} \mathrm{C}$ NMR $(126$ $\left.\mathrm{MHz}, \mathrm{CDCl}_{3}\right) \delta 189.02(\mathrm{t}, J=29.0 \mathrm{~Hz}), 142.71,140.90,129.61,119.70,109.37(\mathrm{t}, J=255.4 \mathrm{~Hz}$ ), 21.29. ${ }^{19} \mathrm{~F}$ NMR $\left(470 \mathrm{MHz}, \mathrm{CDCl}_{3}\right) \delta-122.89(\mathrm{~d}, J=54.1 \mathrm{~Hz}, 2 \mathrm{~F})$. HRMS (GC-APCl) calcd. for $\mathrm{C}_{11} \mathrm{H}_{12} \mathrm{~F}_{2} \mathrm{OS}[\mathrm{M}+]+\mathrm{m} / \mathrm{z} 231.0677$, found 231.0649 .<smiles>O=C(SCCc1ccccc1)C(F)F</smiles>

S-Phenethyl 2,2-difluoroethanethioate (1p). The general method was followed using $2 \mathrm{mmol}$ of the corresponding thiol. This afforded $1 \mathrm{p}$ as a clear oil $\left(379 \mathrm{mg}, 88 \%\right.$ yield). ${ }^{1} \mathrm{H}$ NMR $(700 \mathrm{MHz}$, $\left.\mathrm{CDCl}_{3}\right) \delta 7.34(\mathrm{t}, J=7.5 \mathrm{~Hz}, 2 \mathrm{H}), 7.27(\mathrm{t}, J=6.9 \mathrm{~Hz}, 1 \mathrm{H}), 7.24(\mathrm{~d}, J=7.6 \mathrm{~Hz}, 2 \mathrm{H}), 5.86(\mathrm{t}, J=$ $54.1 \mathrm{~Hz}, 1 \mathrm{H}), 3.26(\mathrm{t}, J=7.7 \mathrm{~Hz}, 2 \mathrm{H}), 2.94(\mathrm{t}, J=7.7 \mathrm{~Hz}, 2 \mathrm{H}) .{ }^{13} \mathrm{C} \mathrm{NMR}\left(176 \mathrm{MHz}, \mathrm{CDCl}_{3}\right) \delta$ $191.32(\mathrm{t}, J=29.0 \mathrm{~Hz}), 139.07,128.66,128.55,126.86,108.98$ (t, $J=254.5 \mathrm{~Hz}), 35.12,29.83$. ${ }^{19} \mathrm{~F}$ NMR $\left(377 \mathrm{MHz}, \mathrm{CDCl}_{3}\right) \delta-123.80(\mathrm{~d}, J=54.1 \mathrm{~Hz}, 2 \mathrm{~F})$. HRMS (ES) calcd. for $\mathrm{C}_{10} \mathrm{H}_{10} \mathrm{~F}_{2} \mathrm{OS}$ $[\mathrm{M}+] \mathrm{m} / \mathrm{z} 216.0420$, found 216.0419 .<smiles>O=C(SCc1ccccc1)C(F)F</smiles>

S-Benzyl 2,2-difluoroethanethioate (1q). The general method was followed using $2 \mathrm{mmol}$ of the corresponding thiol. This afforded $1 \mathrm{q}$ as a clear oil $\left(350 \mathrm{mg}, 87 \%\right.$ yield). ${ }^{1} \mathrm{H} \mathrm{NMR}(700 \mathrm{MHz}$, $\left.\mathrm{CDCl}_{3}\right) \delta 7.39-7.27(\mathrm{~m}, 5 \mathrm{H}), 5.88(\mathrm{t}, J=54.0 \mathrm{~Hz}, 1 \mathrm{H}), 4.25(\mathrm{~s}, 2 \mathrm{H}) .{ }^{13} \mathrm{C} \mathrm{NMR}\left(176 \mathrm{MHz}, \mathrm{CDCl}_{3}\right)$ $\delta 190.99$ (t, $J=29.1 \mathrm{~Hz}), 135.60,128.97,128.85,127.89,109.02(\mathrm{t}, J=254.6 \mathrm{~Hz}), 32.77$ (d, $J=$ $1.5 \mathrm{~Hz}) .{ }^{19} \mathrm{~F}$ NMR $\left(377 \mathrm{MHz}, \mathrm{CDCl}_{3}\right) \delta-123.79(\mathrm{~d}, J=54.0 \mathrm{~Hz}, 2 \mathrm{~F})$. HRMS (ES) calcd. for $\mathrm{C}_{9} \mathrm{H}_{8} \mathrm{~F}_{2} \mathrm{OS}[\mathrm{M}+] \mathrm{m} / \mathrm{z} 202.0264$, found 202.0264 . 
<smiles>O=C(SCc1ccco1)C(F)F</smiles>

S-(Furan-2-ylmethyl) 2,2-difluoroethanethioate (1r). The general method was followed using $3 \mathrm{mmol}$ of the corresponding thiol. This afforded $1 \mathrm{r}$ as a clear oil (467 mg, 81\% yield). ${ }^{1} \mathrm{H}$ NMR $\left(700 \mathrm{MHz}, \mathrm{CDCl}_{3}\right) \delta 7.35(\mathrm{~d}, J=1.0 \mathrm{~Hz}, 1 \mathrm{H}), 6.33-6.29(\mathrm{~m}, 1 \mathrm{H}), 6.29(\mathrm{~d}, J=3.2 \mathrm{~Hz}, 1 \mathrm{H}), 5.88(\mathrm{t}$, $J=54.0 \mathrm{~Hz}, 1 \mathrm{H}), 4.27(\mathrm{~s}, 2 \mathrm{H}) \cdot{ }^{13} \mathrm{C}$ NMR $\left(176 \mathrm{MHz}, \mathrm{CDCl}_{3}\right) \delta 190.52(\mathrm{t}, J=29.4 \mathrm{~Hz}), 148.49$, 142.76, 110.74, 108.99, $108.90(\mathrm{t}, J=254.6 \mathrm{~Hz}), 25.24 .{ }^{19} \mathrm{~F} \mathrm{NMR}\left(377 \mathrm{MHz}, \mathrm{CDCl}_{3}\right) \delta-123.73$ (d, $J=54.0 \mathrm{~Hz}, 2 \mathrm{~F}$ ). HRMS (ES) calcd. for $\mathrm{C}_{7} \mathrm{H}_{6} \mathrm{~F}_{2} \mathrm{O}_{2} \mathrm{~S}[\mathrm{M}+] \mathrm{m} / \mathrm{z}$ 192.0057, found 192.0056 .<smiles>O=C(SC1CCCCC1)C(F)F</smiles>

S-Cyclohexyl 2,2-difluoroethanethioate (1s). The general method was followed using $2 \mathrm{mmol}$ of the corresponding thiol. This afforded $1 \mathrm{~s}$ as a clear oil (341 mg, $88 \%$ yield). ${ }^{1} \mathrm{H}$ NMR $(500 \mathrm{MHz}$, $\left.\mathrm{CDCl}_{3}\right) \delta 5.82(\mathrm{t}, J=54.3 \mathrm{~Hz}, 1 \mathrm{H}), 3.67(\mathrm{dp}, J=10.0,4.0 \mathrm{~Hz}, 1 \mathrm{H}), 2.02-1.93(\mathrm{~m}, 2 \mathrm{H}), 1.74(\mathrm{dt}, J$ $=8.0,4.1 \mathrm{~Hz}, 2 \mathrm{H}), 1.66-1.58(\mathrm{~m}, 1 \mathrm{H}), 1.57-1.42$ (multiple peaks, $4 \mathrm{H}), 1.39-1.27(\mathrm{~m}, 1 \mathrm{H}) .{ }^{13} \mathrm{C}$ $\operatorname{NMR}\left(176 \mathrm{MHz}, \mathrm{CDCl}_{3}\right) \delta 191.03$ (t, $\left.J=28.6 \mathrm{~Hz}\right), 109.03$ (t, $\left.J=254.6 \mathrm{~Hz}\right), 42.51,32.57,25.70$, 25.33. ${ }^{19} \mathrm{~F}$ NMR $\left(377 \mathrm{MHz}, \mathrm{CDCl}_{3}\right) \delta-123.90$ (d, $\left.J=54.3 \mathrm{~Hz}, 2 \mathrm{~F}\right)$. HRMS (ES) calcd. for $\mathrm{C}_{8} \mathrm{H}_{12} \mathrm{~F}_{2} \mathrm{OS}[\mathrm{M}+] \mathrm{m} / \mathrm{z} 194.0577$, found 194.0578 .

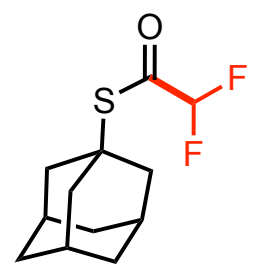

S-((3s,5s,7s)-Adamantan-1-yl) 2,2-difluoroethanethioate (1t). The general method was followed using $2 \mathrm{mmol}$ of the corresponding thiol. This afforded $1 \mathrm{t}$ as a clear oil (397 $\mathrm{mg}, 81 \%$ yield). mp 45.0-47.2 ${ }^{\circ} \mathrm{C} .{ }^{1} \mathrm{H}$ NMR $\left(500 \mathrm{MHz} \mathrm{CDCl}_{3}\right) \delta 5.69(\mathrm{t}, J=54.4 \mathrm{~Hz}, 1 \mathrm{H}), 2.20$ (d, J=2.9 $\mathrm{Hz}, 6 \mathrm{H}), 2.14-2.06(\mathrm{~m}, 3 \mathrm{H}), 1.87-1.67(\mathrm{~m}, 6 \mathrm{H}) .{ }^{13} \mathrm{C} \mathrm{NMR}\left(176 \mathrm{MHz} \mathrm{CDCl}_{3}\right) \delta 190.76(\mathrm{t}, J=27.5$ $\mathrm{Hz}), 108.88$ (t, $J=255.5 \mathrm{~Hz}), 52.42,41.60,36.07,29.83 .{ }^{19} \mathrm{~F} \mathrm{NMR}\left(377 \mathrm{MHz} \mathrm{CDCl}_{3}\right) \delta-123.38$ (d, $J=54.4 \mathrm{~Hz}, 2 \mathrm{~F}$ ). HRMS (ES) calcd. for $\mathrm{C}_{12} \mathrm{H}_{16} \mathrm{~F}_{2} \mathrm{OS}[\mathrm{M}+] \mathrm{m} / \mathrm{z} 246.0890$, found 246.0882 . 
<smiles>COC(C)CCOC(=O)CCSC(=O)C(F)F</smiles>

3-Methoxybutyl 3-((2,2-difluoroacetyl)thio)propanoate (1u). The general method was followed using $2 \mathrm{mmol}$ of the corresponding thiol. This afforded $1 \mathrm{u}$ as a light brown oil (404 $\mathrm{mg}, 75 \%$ yield). ${ }^{1} \mathrm{H}$ NMR $\left(500 \mathrm{MHz}, \mathrm{CDCl}_{3}\right) \delta 5.85(\mathrm{t}, J=54.0 \mathrm{~Hz}, 1 \mathrm{H}), 4.20(\mathrm{t}, J=6.9 \mathrm{~Hz}, 2 \mathrm{H}), 3.39$ (q, $J=6.4$ $\mathrm{Hz}, 1 \mathrm{H}), 3.30(\mathrm{~s}, 3 \mathrm{H}), 3.23(\mathrm{t}, J=7.0 \mathrm{~Hz}, 2 \mathrm{H}), 2.66(\mathrm{t}, J=7.0 \mathrm{~Hz}, 2 \mathrm{H}), 1.83-1.70(\mathrm{~m}, 2 \mathrm{H}), 1.15$ (d, $J=6.0 \mathrm{~Hz}, 3 \mathrm{H}) .{ }^{13} \mathrm{C}$ NMR $\left(126 \mathrm{MHz}, \mathrm{CDCl}_{3}\right) \delta 191.29(\mathrm{t}, J=29.2 \mathrm{~Hz}), 171.01,108.87$ (t, J = 254.6 $\mathrm{Hz}), 73.56,62.10,56.06,35.42,33.59,23.46,18.99 .{ }^{19} \mathrm{~F} \mathrm{NMR}\left(377 \mathrm{MHz}, \mathrm{CDCl}_{3}\right) \delta-123.61$ (d, J $=54.0 \mathrm{~Hz}, 2 \mathrm{~F})$. HRMS (ESI) calcd. for $\mathrm{C}_{10} \mathrm{H}_{16} \mathrm{~F}_{2} \mathrm{O}_{4} \mathrm{~S}[\mathrm{M}+\mathrm{H}] \mathrm{m} / \mathrm{z} 271.0837$, found 271.0830.

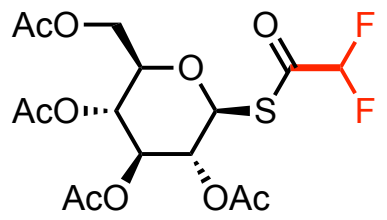

$(2 R, 3 R, 4 S, 5 R, 6 S)-2-($ Acetoxymethyl)-6-((2,2-difluoroacetyl)thio)tetrahydro-2H-pyran-3,4,5triyl triacetate (1w). The general method was followed using $1.5 \mathrm{mmol}$ of the corresponding thiol. Washing, drying, and removal of solvent afforded $1 \mathrm{w}$ as a sticky white solid (543 $\mathrm{mg}, 82 \%$ yield). mp 75.1-78.5 ${ }^{\circ} \mathrm{C} .{ }^{1} \mathrm{H}$ NMR $\left(700 \mathrm{MHz}, \mathrm{CDCl}_{3}\right) \delta 5.87$ (t, $\left.J=53.7 \mathrm{~Hz}, 1 \mathrm{H}\right), 5.24$ (dd, $J=22.8,9.9$ $\mathrm{Hz}, 2 \mathrm{H}), 5.12(\mathrm{t}, J=9.9 \mathrm{~Hz}, 1 \mathrm{H}), 5.06(\mathrm{t}, J=9.8 \mathrm{~Hz}, 1 \mathrm{H}), 4.19(\mathrm{dd}, J=12.6,4.4 \mathrm{~Hz}, 1 \mathrm{H}), 4.03(\mathrm{~d}$, $J=12.5 \mathrm{~Hz}, 1 \mathrm{H}), 3.82(\mathrm{~d}, J=8.3 \mathrm{~Hz}, 1 \mathrm{H}), 2.00(\mathrm{~s}, 3 \mathrm{H}), 1.96(\mathrm{~s}, 3 \mathrm{H}), 1.95(\mathrm{~s}, 3 \mathrm{H}), 1.93(\mathrm{~s}, 3 \mathrm{H})$. ${ }^{13} \mathrm{C}$ NMR $\left(176 \mathrm{MHz}, \mathrm{CDCl}_{3}\right) \delta 188.63(\mathrm{t}, \mathrm{J}=30.3 \mathrm{~Hz}), 170.44,169.87,169.24,169.11,108.57(\mathrm{t}$, $J=255.0 \mathrm{~Hz}), 79.12,76.55,73.58,68.89,67.63,61.45,20.40 .{ }^{19} \mathrm{~F} \mathrm{NMR}\left(376 \mathrm{MHz} \mathrm{CDCl}_{3}\right) \delta-$ 123.26 (dd, $J=308.5,53.7 \mathrm{~Hz}, 1 \mathrm{~F}),-124.66$ (dd, $J=308.5,53.7 \mathrm{~Hz}, 1 \mathrm{~F})$. HRMS (ESI) calcd. for $\mathrm{C}_{9} \mathrm{H}_{8} \mathrm{~F}_{2} \mathrm{OS}[\mathrm{M}+\mathrm{Na}] \mathrm{m} / \mathrm{z}$ 465.0642, found 465.0637. 
VII. Synthesis of $1 \mathrm{v}$

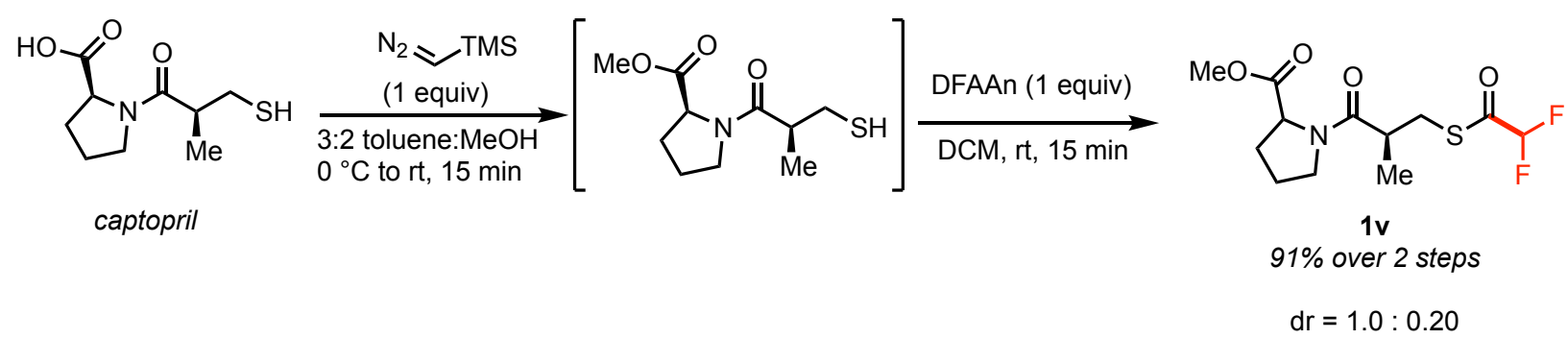

General procedure for the synthesis of methyl ((S)-3-((difluoromethyl)thio)-2methylpropanoyl)prolinate (1v). In an oven dried round bottom flask, captopril (217 mg, 1.0 mmol, 1.0 equiv) was dissolved in $10 \mathrm{~mL}$ of solvent (3:2 anhydrous toluene:MeOH). Under a flow of $\mathrm{N}_{2}$ at $0{ }^{\circ} \mathrm{C}, 2.0 \mathrm{M}$ TMS-diazomethane in diethyl ether $(0.5 \mathrm{~mL}, 1.0 \mathrm{mmol}, 1.0$ equiv) was added dropwise to the stirring reaction until a yellow color persisted in solution. Once the reaction turned yellow, it was allowed to warm to room temperature. The volatiles were then removed in vacuo, leaving behind the methyl ester thiol as a viscous, maroon-colored oil. The oil was dissolved in 6 $\mathrm{mL}$ of $\mathrm{CH}_{2} \mathrm{Cl}_{2}$, and difluoroacetic anhydride was added dropwise $(0.13 \mathrm{~mL}, 1.0 \mathrm{mmol}, 1.0$ equiv) to the solution at room temperature. After stirring the solution at ambient temperature for $15 \mathrm{~min}$, the solution was diluted with $\mathrm{CH}_{2} \mathrm{Cl}_{2}(30 \mathrm{~mL})$ and washed with cold water $(3 \times 10 \mathrm{~mL})$. The organic layer was collected and dried over $\mathrm{MgSO}_{4}$. The solvent was removed in vacuo to afford difluoromethylthioester $\mathbf{1 v}$. During the reaction, isomerization of the tertiary pyrrolidine proton resulted in an inseparable mixture of diastereomers ( $d r=1.0: 0.2)$. Removal of solvent afforded $1 \mathrm{v}$ as a clear, sticky solid (255 mg, 83\% yield, $\mathrm{dr}=1.0: 0.2) . \mathrm{mp} 57.5-60.3^{\circ} \mathrm{C} .{ }^{1} \mathrm{H}$ NMR $(500$ $\left.\mathrm{MHz}, \mathrm{CDCl}_{3}\right) \delta 5.86(\mathrm{t}, J=54.1 \mathrm{~Hz}, 1 \mathrm{H}), 4.54(\mathrm{dd}, J=8.7,4.0 \mathrm{~Hz}, 1 \mathrm{H}), 3.73(\mathrm{~s}, 3 \mathrm{H}), 3.67-3.50$ $(\mathrm{m}, 2 \mathrm{H}), 3.26$ (dd, $J=13.4,8.4 \mathrm{~Hz}, 1 \mathrm{H}), 3.13$ (dd, $J=13.6,5.9 \mathrm{~Hz}, 1 \mathrm{H}), 2.85(\mathrm{~h}, J=6.9 \mathrm{~Hz}, 1 \mathrm{H})$, 2.32-2.14 (m, 1H), 2.08 (ddd, $J=14.1,6.9,2.3 \mathrm{~Hz}, 1 \mathrm{H}), 2.05-1.96(\mathrm{~m}, 2 \mathrm{H}), 1.30$ (d, $J=6.9 \mathrm{~Hz}$, $3 \mathrm{H})$ for the major diastereomer. ${ }^{13} \mathrm{C}$ NMR $\left(126 \mathrm{MHz}, \mathrm{CDCl}_{3}\right) \delta 191.91(\mathrm{t}, \mathrm{J}=29.0 \mathrm{~Hz}), 172.75$, 172.54, $108.92(t, J=254.6 \mathrm{~Hz}), 58.67,52.21,46.90,38.07,31.25,29.00,24.82,16.85$ for the major diastereomer. ${ }^{19} \mathrm{~F}$ NMR (471 $\left.\mathrm{MHz}, \mathrm{CDCl}_{3}\right) \delta-122.91$ to -125.63 (multiple peaks, $2 \mathrm{~F}$ ). HRMS (ESI) calcd. for $\mathrm{C}_{9} \mathrm{H}_{8} \mathrm{~F}_{2} \mathrm{OS}[\mathrm{M}+\mathrm{H}] \mathrm{m} / \mathrm{z} 310.0946$, found 310.0919 . 


\section{Synthesis of fluoroalkyl thioesters}

General procedure for the synthesis of fluoroalkylated thioesters from fluoroalkyl carboxylic acids (Method $A)$. The respective thiol $(2.0 \mathrm{mmol}, 1.0$ equiv), fluorocarboxylic acid (2.2 mmol, 1.1 equiv), EDC (3.0 mmol, 1.5 equiv), pyridine ( $2.0 \mathrm{mmol}, 1$ equiv), and DMAP (0.2 mmol, 0.1 equiv) were weighed into a $20 \mathrm{~mL}$ vial and dissolved in $\mathrm{CH}_{2} \mathrm{Cl}_{2}(6 \mathrm{~mL})$. The reaction was stirred at room temperature for $20 \mathrm{~h}$. After this time, the solution was diluted with $\mathrm{CH}_{2} \mathrm{Cl}_{2}(20$ $\mathrm{mL})$ and washed with cold water $(3 \times 10 \mathrm{~mL})$. The organic layer was collected and dried over $\mathrm{MgSO}_{4}$. Solvent was removed in vacuo to afford the crude reaction mixture. Purification was performed by silica column chromatography using an ethyl acetate/hexane solvent mixture.<smiles>COc1ccc(SC(=O)CF)cc1</smiles>

S-(4-Methoxyphenyl) 2-fluoroethanethioate (1x). Method A was followed using 4methoxythiophenol $(1.0 \mathrm{mmol})$ and 2-fluoroacetic acid $(1.1 \mathrm{mmol})$. Purification by flash chromatography on silica gel (hexanes/EtOAc, 95:5) afforded $1 \mathbf{x}$ as a colorless oil (162 $\mathrm{mg}, 81 \%$ yield). ${ }^{1} \mathrm{H}$ NMR $\left(400 \mathrm{MHz}, \mathrm{CDCl}_{3}\right) \delta 7.33(\mathrm{~d}, J=7.8 \mathrm{~Hz}, 2 \mathrm{H}), 6.96(\mathrm{~d}, J=7.8 \mathrm{~Hz}, 2 \mathrm{H}), 4.96(\mathrm{~d}, J=$ $47.1 \mathrm{~Hz}, 2 \mathrm{H}), 3.81$ (s, 3H). ${ }^{13} \mathrm{C}$ NMR (126 MHz, $\left.\mathrm{CDCl}_{3}\right) \delta 196.49$ (d, J = $\left.24.9 \mathrm{~Hz}\right), 161.19,136.54$, $115.62(\mathrm{~d}, J=4.9 \mathrm{~Hz}), 115.31,84.87(\mathrm{~d}, J=187.9 \mathrm{~Hz}), 55.50 .{ }^{19} \mathrm{~F} \mathrm{NMR}\left(377 \mathrm{MHz}, \mathrm{CDCl}_{3}\right) \delta-$ $225.59(\mathrm{t}, J=47.1 \mathrm{~Hz}, 1 \mathrm{~F})$. HRMS (ESI) calcd. for $\mathrm{C}_{9} \mathrm{H}_{10} \mathrm{FO}_{2} \mathrm{~S}[\mathrm{M}+\mathrm{H}] \mathrm{m} / \mathrm{z} 201.0386$, found 201.0389 .

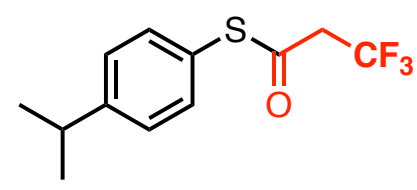

S-(4-Isopropylphenyl) 3,3,3-trifluoropropanethioate (1y). Method A was followed using 4isopropylthiophenol $(2.0 \mathrm{mmol})$ and 3,3,3-trifluoropropionic acid $(2.2 \mathrm{mmol})$. Purification by flash chromatography on silica gel (hexanes/EtOAc, 95:5) afforded $1 \mathrm{y}$ as a colorless oil (398 mg, 76\% yield). ${ }^{1} \mathrm{H}$ NMR $\left(500 \mathrm{MHz}, \mathrm{CDCl}_{3}\right) \delta 7.36(\mathrm{~d}, J=8.3 \mathrm{~Hz}, 2 \mathrm{H}), 7.32(\mathrm{~d}, J=8.3 \mathrm{~Hz}, 2 \mathrm{H}), 3.45(\mathrm{q}, J=$ $9.9 \mathrm{~Hz}, 2 \mathrm{H}$ ), 2.96 (septet, $J=6.9 \mathrm{~Hz}, 1 \mathrm{H}), 1.28(\mathrm{~d}, J=6.9 \mathrm{~Hz}, 6 \mathrm{H}) .{ }^{13} \mathrm{C} \mathrm{NMR}\left(176 \mathrm{MHz}, \mathrm{CDCl}_{3}\right) \delta$ $187.63(\mathrm{q}, J=3.1 \mathrm{~Hz}), 151.28,134.41,127.70,122.94(\mathrm{~d}, J=277.8 \mathrm{~Hz}), 122.78,46.42(\mathrm{q}, J=$ 29.6 Hz), 33.99, 23.73. ${ }^{19} \mathrm{~F}$ NMR (377 MHz, $\left.\mathrm{CDCl}_{3}\right) \delta-62.66(\mathrm{t}, J=9.9 \mathrm{~Hz}, 3 \mathrm{~F})$. HRMS (ESI) calcd. for $\mathrm{C}_{12} \mathrm{H}_{14} \mathrm{~F}_{3} \mathrm{OS}[\mathrm{M}+\mathrm{H}] \mathrm{m} / \mathrm{z} 263.0717$, found 263.0717 . 


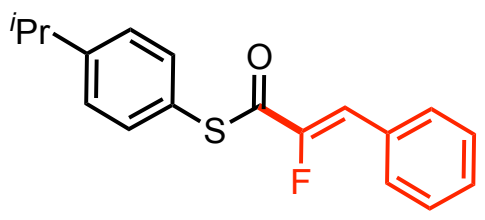

S-(4-Isopropylphenyl)-(Z)-2-fluoro-3-phenylprop-2-enethioate (1ab). The general method was followed using 4-isopropylthiophenol $(2.0 \mathrm{mmol})$ and (Z)-2-fluoro-3-phenylacrylic acid $(2.2$ $\mathrm{mmol}$ ). Purification by flash chromatography on silica gel (hexanes/EtOAc, 95:5) afforded $\mathbf{1} \mathbf{a b}$ as a white solid (480 mg, 80\% yield). $\mathrm{mp} 92-94{ }^{\circ} \mathrm{C} .{ }^{1} \mathrm{H}$ NMR (500 MHz, $\left.\mathrm{CDCl}_{3}\right) \delta 7.70$ (dd, $J=7.9$, $1.8 \mathrm{~Hz}, 2 \mathrm{H}$ ), 7.48-7.38 (multiple peaks, $5 \mathrm{H}$ ), $7.34(\mathrm{~d}, J=8.2 \mathrm{~Hz}, 2 \mathrm{H}), 6.86(\mathrm{~d}, J=36.6 \mathrm{~Hz}, 1 \mathrm{H})$, 2.97 (septet, $J=6.9 \mathrm{~Hz}, 1 \mathrm{H}), 1.30(\mathrm{~d}, J=6.9 \mathrm{~Hz}, 6 \mathrm{H}) .{ }^{13} \mathrm{C} \mathrm{NMR}\left(126 \mathrm{MHz}, \mathrm{CDCl}_{3}\right) \delta 185.51$ (d, $J$ $=37.5 \mathrm{~Hz}), 152.31(\mathrm{~d}, J=271.8 \mathrm{~Hz}), 151.13,135.09,131.04(\mathrm{~d}, J=8.1 \mathrm{~Hz}), 130.99,130.27(\mathrm{~d}$, $J=2.6 \mathrm{~Hz}), 129.14,127.86,122.79(\mathrm{~d}, J=4.4 \mathrm{~Hz}), 114.03(\mathrm{~d}, J=4.4 \mathrm{~Hz}), 34.23,24.03 .{ }^{19} \mathrm{~F}$ NMR $\left(471 \mathrm{MHz}, \mathrm{CDCl}_{3}\right) \delta-125.26$ (d, $J=36.6 \mathrm{~Hz}, 1 \mathrm{~F}$ ). HRMS (ESI) calcd. for $\mathrm{C}_{18} \mathrm{H}_{18} \mathrm{FOS}[\mathrm{M}+\mathrm{H}] \mathrm{m} / \mathrm{z}$ 301.1062, found 301.1065 .

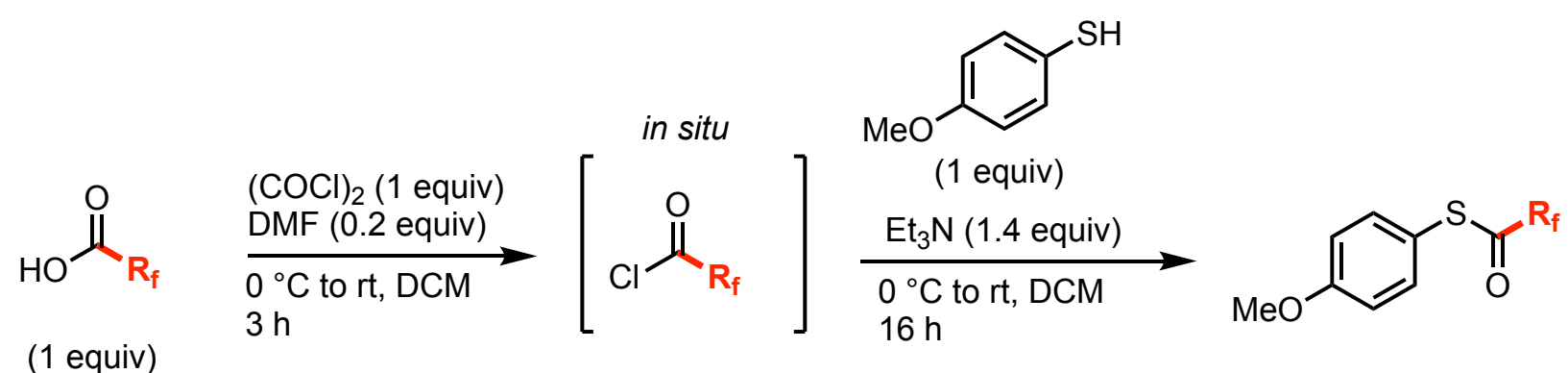

Fluoroalkyl thioester synthesis via in situ generated acid chlorides (Method B). ${ }^{2}$ The corresponding carboxylic acid (4.25 mmol, 1 equiv) was dissolved in $8 \mathrm{~mL}$ of $\mathrm{CH}_{2} \mathrm{Cl}_{2}$ and DMF $\left(0.07 \mathrm{~mL}, 0.85 \mathrm{mmol}, 0.2\right.$ equiv) was added. At $0{ }^{\circ} \mathrm{C},(\mathrm{COCl})_{2}$ was added dropwise $(0.36 \mathrm{~mL}, 4.25$ $\mathrm{mmol}, 1$ equiv) to the stirring solution. The reaction was then allowed to warm to room temperature and stir for $3 \mathrm{~h}$. After this time, the acid chloride solution was added dropwise to a $0{ }^{\circ} \mathrm{C}$ solution of thiol (595 mg, $4.25 \mathrm{mmol}, 1$ equiv) and $\mathrm{Et}_{3} \mathrm{~N}\left(0.83 \mathrm{~mL}, 6 \mathrm{mmol}, 1.4\right.$ equiv) in $8 \mathrm{~mL}$ of $\mathrm{CH}_{2} \mathrm{Cl}_{2}$. The resulting mixture was allowed to warm to room temperature and stir overnight, after which the volatiles were removed in vacuo. The thioester product was purified via flash column chromatography on silica gel. 
<smiles>COc1ccc(SC(=O)C(F)C(F)(F)F)cc1</smiles>

S)-(4-Methoxyphenyl)(1,2,2,2-tetrafluoroethyl)sulfane (1z). Method B was followed using 4(methoxy)thiophenol (3.5 mmol) and 2,3,3,3-(tetrafluoro)propionic acid (3.5 mmol). Purification by flash chromatography on silica gel (hexanes/EtOAc, 90:10) afforded $1 \mathbf{z}$ as a clear oil (591 mg, $63 \%$ yield). ${ }^{1} \mathrm{H}$ NMR (401 MHz, $\left.\mathrm{CDCl}_{3}\right) \delta 7.32(\mathrm{~d}, J=8.9 \mathrm{~Hz}, 1 \mathrm{H}), 6.97(\mathrm{~d}, J=8.9 \mathrm{~Hz}, 1 \mathrm{H}), 5.16$ (dq, $J=46.1,6.4 \mathrm{~Hz}, 1 \mathrm{H}$ ), $3.83(\mathrm{~s}, 2 \mathrm{H}) .{ }^{13} \mathrm{C} \mathrm{NMR}\left(126 \mathrm{MHz}, \mathrm{CDCl}_{3}\right) \delta 190.31$ (d, $J=27.3 \mathrm{~Hz}$ ), 161.41, 136.29, $119.95(\mathrm{dd}, J=283.1,25.7 \mathrm{~Hz}), 115.35,114.29(\mathrm{~d}, J=5.3 \mathrm{~Hz}), 88.94(\mathrm{dq}, J=$ 204.3, 33.9 Hz), 55.39. $\left.{ }^{19} \mathrm{~F} \mathrm{NMR} \mathrm{(377} \mathrm{MHz,} \mathrm{CDCl}_{3}\right) \delta-75.70$ (dd, J = 11.8, 6.4 Hz, 3F), -202.79 (dq, $J=46.1,11.8 \mathrm{~Hz}, 1 \mathrm{~F}$ ). HRMS (GC-APCI) calcd. for $\mathrm{C}_{10} \mathrm{H}_{8} \mathrm{~F}_{4} \mathrm{O}_{2} \mathrm{~S}[\mathrm{M}+\mathrm{H}]+\mathrm{m} / \mathrm{z} 269.0281$, found 269.0262 .<smiles>COc1ccc(SC(=O)C(F)(F)c2ccccc2)cc1</smiles>

S-(4-Isopropylphenyl) 2,2-difluoro-2-phenylethanethioate (1aa). Method B was followed using 4isopropylthiophenol $(1.0 \mathrm{mmol})$ and 2,2-difluoro-2-phenylacetic acid $(1.1 \mathrm{mmol})$. Purification by flash chromatography on silica gel (hexanes/EtOAc, 95:5) afforded 1aa as a colorless oil (199 mg, $65 \%$ yield). $\mathrm{Mp} 75.0^{\circ} \mathrm{C}-77.6^{\circ} \mathrm{C}^{1} \mathrm{H}$ NMR $\left(500 \mathrm{MHz}, \mathrm{CDCl}_{3}\right) \delta 7.68$ (d, J = 7.0 Hz, 2H), 7.557.47 (multiple peaks, 3H), $7.32(\mathrm{~d}, J=8.8 \mathrm{~Hz}, 2 \mathrm{H}), 6.96(\mathrm{~d}, J=8.8 \mathrm{~Hz}, 2 \mathrm{H}), 3.83(\mathrm{~s}, 3 \mathrm{H}) .{ }^{13} \mathrm{C} \mathrm{NMR}$ $\left(176 \mathrm{MHz}, \mathrm{CDCl}_{3}\right) \delta 191.98(\mathrm{t}, J=36.8 \mathrm{~Hz}), 161.19,136.16,132.65,132.07(\mathrm{t}, J=25.3 \mathrm{~Hz})$, 131.12, 128.68, $125.72(\mathrm{t}, J=6.1 \mathrm{~Hz}), 116.01(\mathrm{t}, J=256.7 \mathrm{~Hz}), 115.18,55.38 .{ }^{19} \mathrm{~F}$ NMR $(471$ $\left.\mathrm{MHz}, \mathrm{CDCl}_{3}\right) \delta-101.48$. HRMS (ESI) calcd. for $\mathrm{C}_{15} \mathrm{H}_{12} \mathrm{~F}_{2} \mathrm{O}_{2} \mathrm{~S}[\mathrm{M}+\mathrm{H}]+\mathrm{m} / \mathrm{z} 295.0626$, found 295.0599 . 


\section{Synthesis of fluoroalkyl thioethers}

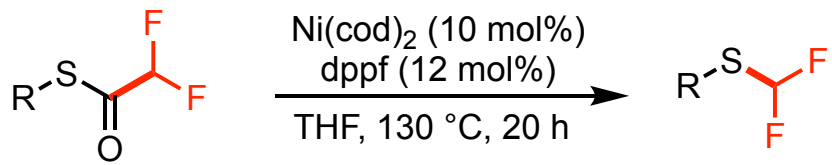

General procedure for the decarbonylation of fluorinated thioesters: $\mathrm{Ni}(\mathrm{cod})_{2}(13.8 \mathrm{mg}, 0.05$ mmol, 0.1 equiv) and dppf (30.9 mg, $0.06 \mathrm{mmol}, 0.12$ equiv) were dissolved in anhydrous THF (1 $\mathrm{mL}$ ), and this mixture was stirred for $15 \mathrm{~min}$ at room temperature. The resulting solution was then transferred to a tall $10 \mathrm{~mL}$ vial with a stir bar containing the respective, pre-weighed thioester $(0.5$ mmol, 1 equiv). The vial was sealed with a Teflon-lined screw cap and taken outside the glovebox. The vial was heated at $130^{\circ} \mathrm{C}$ for $20 \mathrm{~h}$ with stirring. The reaction was then allowed to cool to room temperature, and the solvent was removed in vacuo. The resulting crude brown residue was purified via flash chromatography on silica gel.<smiles>FC(F)Sc1ccccc1</smiles>

(Difluoromethyl)(phenyl)sulfane (2a). The general decarbonylation procedure was followed using $0.5 \mathrm{mmol}$ of the corresponding thioester. Purification by flash chromatography on silica gel (hexanes/EtOAc, 97:3) afforded the product as a clear oil (49 mg, 61\% yield). ${ }^{1} \mathrm{H} \mathrm{NMR}(500 \mathrm{MHz}$, $\left.\mathrm{CDCl}_{3}\right) \delta 7.60(\mathrm{~d}, J=7.6 \mathrm{~Hz}, 2 \mathrm{H}$ ), 7.48-7.37 (multiple peaks, $3 \mathrm{H}), 6.85(\mathrm{t}, J=57.0 \mathrm{~Hz}, 1 \mathrm{H}) .{ }^{13} \mathrm{C}$ $\operatorname{NMR}\left(176 \mathrm{MHz}, \mathrm{CDCl}_{3}\right) \delta 135.30,129.74,129.34,126.10,120.99(\mathrm{t}, \mathrm{J}=275.0 \mathrm{~Hz}) .{ }^{19} \mathrm{~F} \mathrm{NMR}(377$ $\mathrm{MHz}_{\mathrm{CDCl}}$ ) $\delta-91.23\left(\mathrm{~d}, J=57.0 \mathrm{~Hz}, 2 \mathrm{~F}\right.$ ). HRMS (EI) calcd. for $\mathrm{C}_{7} \mathrm{H}_{6} \mathrm{~F}_{2} \mathrm{~S}[\mathrm{M}+] \mathrm{m} / \mathrm{z} 160.0158$, found 160.0163 .<smiles>COc1ccc(SC(F)F)cc1</smiles>

(Difluoromethyl)(4-methoxyphenyl)sulfane (2b). The general decarbonylation procedure was followed using $0.5 \mathrm{mmol}$ of the corresponding thioester. Purification by flash chromatography on silica gel (hexanes/EtOAc, 97:3) afforded the product as a clear oil (65 mg, 69\% yield). ${ }^{1} \mathrm{H}$ NMR $\left(500 \mathrm{MHz} \mathrm{CDCl}_{3}\right) \delta 7.52(\mathrm{~d}, J=8.7 \mathrm{~Hz}, 2 \mathrm{H}), 6.91(\mathrm{~d}, J=8.6 \mathrm{~Hz}, 2 \mathrm{H}), 6.75(\mathrm{t}, J=57.2 \mathrm{~Hz}, 1 \mathrm{H})$, $3.83(\mathrm{~s}, 3 \mathrm{H}) .{ }^{13} \mathrm{C}$ NMR $\left(176 \mathrm{MHz}, \mathrm{CDCl}_{3}\right) \delta 161.17,137.57,120.94(\mathrm{t}, \mathrm{J}=275.1 \mathrm{~Hz}), 116.15$, 
114.89, 55.36. ${ }^{19} \mathrm{~F}$ NMR (471 MHz, $\left.\mathrm{CDCl}_{3}\right) \delta-105.48(\mathrm{~d}, J=57.2 \mathrm{~Hz}, 2 \mathrm{~F})$. HRMS (GC-APCl) calcd. for $\mathrm{C}_{8} \mathrm{H}_{8} \mathrm{~F}_{2} \mathrm{~S}[\mathrm{M}+] \mathrm{m} / \mathrm{z} 190.0264$, found 190.0258 .

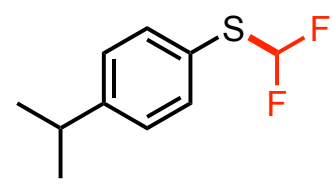

(Difluoromethyl)(4-isopropylphenyl)sulfane (2c). The general decarbonylation procedure was followed using $0.5 \mathrm{mmol}$ of the corresponding thioester. Purification by flash chromatography on silica gel (hexanes/EtOAc, 97:3) afforded the product as a clear oil (65 mg, 64\% yield). ${ }^{1} \mathrm{H}$ NMR $\left(700 \mathrm{MHz}, \mathrm{CDCl}_{3}\right) \delta 7.51(\mathrm{~d}, J=8.0 \mathrm{~Hz}, 2 \mathrm{H}), 7.25(\mathrm{~d}, J=7.8 \mathrm{~Hz}, 2 \mathrm{H}), 6.80(\mathrm{t}, J=57.1 \mathrm{~Hz}, 1 \mathrm{H})$, $2.93(\mathrm{p}, J=6.9 \mathrm{~Hz}, 1 \mathrm{H}), 1.26(\mathrm{~d}, J=6.9 \mathrm{~Hz}, 6 \mathrm{H}) .{ }^{13} \mathrm{C} \mathrm{NMR}\left(176 \mathrm{MHz}, \mathrm{CDCl}_{3}\right) \delta 150.97,135.58$, 127.55, $121.17(\mathrm{t}, J=274.8 \mathrm{~Hz}), 33.91,23.79 .{ }^{19} \mathrm{~F} \mathrm{NMR}\left(377 \mathrm{MHz}, \mathrm{CDCl}_{3}\right) \delta-91.81(\mathrm{~d}, J=57.1$ $\mathrm{Hz}, 2 \mathrm{~F}$ ). HRMS (GC-APCl) calcd. for $\mathrm{C}_{10} \mathrm{H}_{12} \mathrm{~F}_{2} \mathrm{~S}[\mathrm{M}+] \mathrm{m} / \mathrm{z} 202.0628$, found 202.0622.

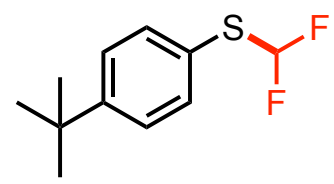

(4-(Tert-butyl)phenyl)(difluoromethyl)sulfane (2d). The general decarbonylation procedure was followed using $0.5 \mathrm{mmol}$ of the corresponding thioester. Purification by flash chromatography on silica gel (hexanes/EtOAc, 97:3) afforded the product as a clear oil (55 mg, $51 \%$ yield). ${ }^{1} \mathrm{H}$ NMR $\left(500 \mathrm{MHz}, \mathrm{CDCl}_{3}\right) \delta 7.53(\mathrm{~d}, J=8.4 \mathrm{~Hz}, 2 \mathrm{H}), 7.43(\mathrm{~d}, J=8.2 \mathrm{~Hz}, 2 \mathrm{H}), 6.82(\mathrm{t}, J=57.2 \mathrm{~Hz}$, 1H), 1.35 (s, 9H). ${ }^{13} \mathrm{C} \mathrm{NMR}\left(126 \mathrm{MHz}, \mathrm{CDCl}_{3}\right) \delta 153.22,135.19,126.45,122.53,121.19$ (t, $J=$ $274.9 \mathrm{~Hz}$ ), 34.75, 31.16. ${ }^{19} \mathrm{~F}$ NMR $\left(377 \mathrm{MHz}, \mathrm{CDCl}_{3}\right) \delta-91.79$ (d, $\left.J=57.2 \mathrm{~Hz}, 2 \mathrm{~F}\right)$. HRMS (GCAPCl) calcd. for $\mathrm{C}_{11} \mathrm{H}_{14} \mathrm{~F}_{2} \mathrm{~S}[\mathrm{M}+] \mathrm{m} / \mathrm{z} 216.0784$, found 216.0779 .<smiles>CN(C)c1ccc(SC(F)F)cc1</smiles>

4-((Difluoromethyl)thio)- $\mathbf{N}, \mathbf{N}$-dimethylaniline (2e). The general decarbonylation procedure was followed using $0.5 \mathrm{mmol}$ of the corresponding thioester. Purification by flash chromatography on silica gel (hexanes/EtOAc, 90:10) afforded the purified product as a yellow oil (99 $\mathrm{mg}, 98 \%$ yield). ${ }^{1} \mathrm{H}$ NMR $\left(700 \mathrm{MHz}, \mathrm{CDCl}_{3}\right) \delta 7.54(\mathrm{~d}, J=8.9 \mathrm{~Hz}, 2 \mathrm{H}), 6.81(\mathrm{t}, J=57.5 \mathrm{~Hz}, 1 \mathrm{H}), 6.79$ (d, J = 8.5 $\mathrm{Hz}, 2 \mathrm{H}), 3.11(\mathrm{~s}, 6 \mathrm{H}) .{ }^{13} \mathrm{C} \mathrm{NMR}\left(176 \mathrm{MHz}, \mathrm{CDCl}_{3}\right) \delta 151.55,137.49,121.52(\mathrm{t}, J=274.9 \mathrm{~Hz})$, 
112.61, 109.96, 40.28. $\left.{ }^{19} \mathrm{~F} \mathrm{NMR} \mathrm{(471} \mathrm{MHz,} \mathrm{CDCl}_{3}\right) \delta-92.60(\mathrm{~d}, J=57.5 \mathrm{~Hz}, 2 \mathrm{~F})$. HRMS (ESI) calcd. for $\mathrm{C}_{9} \mathrm{H}_{11} \mathrm{~F}_{2} \mathrm{NS}[\mathrm{M}+\mathrm{H}]+\mathrm{m} / \mathrm{z} 204.0680$, found 204.0653 .<smiles>FC(F)Sc1ccc2ccccc2c1</smiles>

(Difluoromethyl)(naphthalen-2-yl)sulfane (2f). The general decarbonylation procedure was followed using $0.5 \mathrm{mmol}$ of the corresponding thioester. Purification by flash chromatography on silica gel (hexanes/EtOAc, 97:3) afforded the product as a light brown oil (82 mg, $86 \%$ yield). ${ }^{1} \mathrm{H}$ NMR $\left(500 \mathrm{MHz}, \mathrm{CDCl}_{3}\right) \delta 8.13$ (s, 1H), 7.90-7.81 (multiple peaks, 3H), $7.63(\mathrm{~d}, J=8.5 \mathrm{~Hz}, 1 \mathrm{H}$ ), $7.56(\mathrm{ddd}, J=6.8,3.7,1.8 \mathrm{~Hz}, 2 \mathrm{H}), 6.92(\mathrm{t}, J=56.9 \mathrm{~Hz}, 1 \mathrm{H}) .{ }^{13} \mathrm{C} \mathrm{NMR}\left(176 \mathrm{MHz}, \mathrm{CDCl}_{3}\right) \delta$ $135.41,133.50,133.42,131.38,129.04,127.92,127.76,127.40,126.88,123.30,121.12(\mathrm{t}, J=$ $275.3 \mathrm{~Hz}) .{ }^{19} \mathrm{~F} \mathrm{NMR}\left(377 \mathrm{MHz}, \mathrm{CDCl}_{3}\right) \delta-91.59(\mathrm{~d}, J=56.9 \mathrm{~Hz}, 2 \mathrm{~F})$. HRMS (GC-APCl) calcd. for $\mathrm{C}_{11} \mathrm{H}_{8} \mathrm{~F}_{2} \mathrm{~S}[\mathrm{M}+] \mathrm{m} / \mathrm{z} 210.0315$, found 210.0309 .<smiles>Fc1ccc(SC(F)F)cc1</smiles>

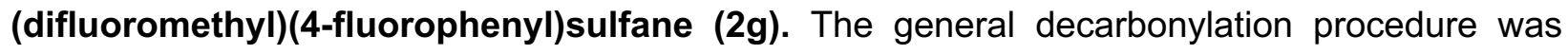
followed using $0.3 \mathrm{mmol}$ of the corresponding thioester, and the yield was determined by ${ }^{19} \mathrm{~F} \mathrm{NMR}$ spectroscopy with 4-fluorotoluene as the internal standard (88\% yield). ${ }^{19} \mathrm{~F}$ NMR shifts were consistent with formation of decarbonylated (difluoromethyl)thioether, ${ }^{19} \mathrm{~F} N \mathrm{NM}\left(377 \mathrm{~Hz}, \mathrm{CDCl}_{3}\right)$ $\delta 91.50 \mathrm{ppm}(\mathrm{d}, J=56.7 \mathrm{~Hz}, 2 \mathrm{~F})$. Due to the high volatility of the product, attempts at isolation were unsuccessful.<smiles>COC(=O)c1ccc(SC(F)F)cc1</smiles>

methyl 4-((difluoromethyl)thio)benzoate (2h). The general decarbonylation procedure was followed using $0.3 \mathrm{mmol}$ of the corresponding thioester, and the yield was determined by ${ }^{19} \mathrm{~F} N \mathrm{NMR}$ spectroscopy with 4-fluorotoluene as the internal standard (54\% yield). ${ }^{19} \mathrm{~F}$ NMR shifts were consistent with formation of decarbonylated (difluoromethyl)thioether, ${ }^{19} \mathrm{~F} \mathrm{NMR}\left(471 \mathrm{~Hz}, \mathrm{CDCl}_{3}\right)$ $\delta-92.13 \mathrm{ppm}(\mathrm{d}, J=56.5 \mathrm{~Hz}, 2 \mathrm{~F})$. 
<smiles>O=C(Nc1ccc(SC(F)F)cc1)C(F)(F)F</smiles>

$\mathbf{N}$-(4-((Difluoromethyl)thio)phenyl)-2,2-difluoroacetamide (2i). The general decarbonylation procedure was followed using $0.5 \mathrm{mmol}$ of the corresponding thioester. Purification by flash chromatography on silica gel (hexanes/EtOAc, 80:20) afforded the product as a white solid (19 $\mathrm{mg}, 15 \%$ yield). During the work up, the acetyl group was cleaved, affording the difluoromethylacetamide as the isolated product. $\mathrm{mp} 101.8-102.8^{\circ} \mathrm{C}$. ${ }^{1} \mathrm{H} \mathrm{NMR}\left(500 \mathrm{MHz}, \mathrm{CDCl}_{3}\right)$ $\delta 8.03(\mathrm{~b}, 1 \mathrm{H}), 7.63(\mathrm{~d}, J=8.5 \mathrm{~Hz}, 2 \mathrm{H}), 7.60(\mathrm{~d}, J=8.3 \mathrm{~Hz}, 2 \mathrm{H}), 6.80(\mathrm{t}, J=56.9 \mathrm{~Hz}, 1 \mathrm{H}), 6.03(\mathrm{t}$, $J=54.3 \mathrm{~Hz}, 1 \mathrm{H}) .{ }^{13} \mathrm{C}$ NMR $\left(176 \mathrm{MHz}, \mathrm{CDCl}_{3}\right) \delta 160.39(\mathrm{t}, J=24.7 \mathrm{~Hz}), 137.25,136.65,122.58$, 120.77, 120.45 (t, $J=275.7 \mathrm{~Hz}), 108.36$ (t, $J=254.4 \mathrm{~Hz}) .{ }^{19} \mathrm{~F} \mathrm{NMR}\left(471 \mathrm{MHz}, \mathrm{CDCl}_{3}\right) \delta-87.24$ (d, $J=56.9 \mathrm{~Hz}, 1 \mathrm{~F}),-121.02$ (d, $J=54.3 \mathrm{~Hz}, 1 \mathrm{~F})$. HRMS (GC-APCI) calcd. for $\mathrm{C}_{9} \mathrm{H}_{7} \mathrm{~F}_{4} \mathrm{OS}[\mathrm{M}+\mathrm{H}]$ $\mathrm{m} / \mathrm{z} 254.0284$, found 254.0265 .<smiles>Fc1ccccc1SC(F)F</smiles>

(difluoromethyl)(2-fluorophenyl)sulfane (2j). The general decarbonylation procedure was followed using $0.3 \mathrm{mmol}$ of the corresponding thioester, and the yield was determined by ${ }^{19} \mathrm{~F}$ NMR spectroscopy with 4-fluorotoluene as the internal standard ( $36 \%$ yield). ${ }^{19} \mathrm{~F} \mathrm{NMR}$ shifts were consistent with formation of decarbonylated (difluoromethyl)thioether, ${ }^{19} \mathrm{~F} \mathrm{NMR}\left(377 \mathrm{~Hz}, \mathrm{CDCl}_{3}\right)$ $-91.17 \mathrm{ppm}(\mathrm{d}, J=56.9 \mathrm{~Hz}, 2 \mathrm{~F})$.). Due to the high volatility of the product, attempts at isolation were unsuccessful.<smiles>FC(F)Sc1ccc(C(F)(F)F)cc1</smiles>

(Difluoromethyl)(4-(trifluoromethyl)phenyl)sulfane (2k). The general decarbonylation procedure was followed using $0.15 \mathrm{mmol}$ of the corresponding thioester. The final yield (33\%) was determined by ${ }^{19} \mathrm{~F}$ NMR spectroscopic analysis with 4-fluorotoluene as an internal standard. ${ }^{19} \mathrm{~F}$ NMR shifts were consistent with formation of decarbonylated (difluoromethyl)thioether. ${ }^{19} \mathrm{~F}$ $\operatorname{NMR}\left(377 \mathrm{~Hz}, \mathrm{CDCl}_{3}\right) \delta-61.77$ (s, 3F), $-91.32 \mathrm{ppm}(\mathrm{d}, J=56.0 \mathrm{~Hz}, 2 \mathrm{~F})$. 


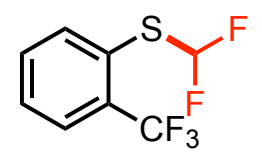

(Difluoromethyl)(4-(trifluoromethyl)phenyl)sulfane (2I). The general decarbonylation procedure was followed using $0.15 \mathrm{mmol}$ of the corresponding thioester. The final yield (38\%) was determined by ${ }^{19} \mathrm{~F}$ NMR spectroscopic analysis with 4-fluorotoluene as an internal standard. ${ }^{19} \mathrm{~F}$ NMR shifts were consistent with formation of decarbonylated (difluoromethyl)thioether. ${ }^{19} \mathrm{~F}$ $\operatorname{NMR}\left(377 \mathrm{~Hz}, \mathrm{CDCl}_{3}\right) \delta-58.59$ (s, 3F), $-91.22 \mathrm{ppm}(\mathrm{d}, J=55.8 \mathrm{~Hz}, 2 \mathrm{~F})$.<smiles>O=[N+]([O-])c1ccccc1SC(F)F</smiles>

(Difluoromethyl)(o-tolyl)sulfane $(\mathbf{2 m})$. The general decarbonylation procedure was followed using $0.5 \mathrm{mmol}$ of the corresponding thioester. Purification by flash chromatography on silica gel (hexanes/EtOAc, 97:3) afforded the product as a clear oil (69 mg, 79\% yield). ${ }^{1} \mathrm{H}$ NMR (700 MHz, $\left.\mathrm{CDCl}_{3}\right) \delta 7.58(\mathrm{~d}, J=7.7 \mathrm{~Hz}, 1 \mathrm{H}), 7.35-7.28(\mathrm{~m}, 2 \mathrm{H}), 7.21(\mathrm{t}, J=7.5 \mathrm{~Hz}, 1 \mathrm{H}), 6.79(\mathrm{t}, J=56.9 \mathrm{~Hz}$, 1H), 2.51 (s, 3H). ${ }^{13} \mathrm{C}$ NMR (176 MHz, $\left.\mathrm{CDCl}_{3}\right)$ 142.97, 136.83, 130.87, 130.18, 126.77, 125.53, $121.23(\mathrm{t}, J=275.0 \mathrm{~Hz}), 21.24 .{ }^{19} \mathrm{~F} \mathrm{NMR}\left(377 \mathrm{MHz}, \mathrm{CDCl}_{3}\right) \delta-91.32(\mathrm{~d}, J=56.9 \mathrm{~Hz}, 2 \mathrm{~F})$. HRMS (GC-APCl) calcd. for $\mathrm{C}_{8} \mathrm{H}_{8} \mathrm{~F}_{2} \mathrm{~S}[\mathrm{M}+] \mathrm{m} / \mathrm{z}$ 174.0315, found 174.0309 .<smiles>CC(C)c1ccccc1SC(F)F</smiles>

(Difluoromethyl)(2-isopropylphenyl)sulfane (2n). The general decarbonylation procedure was followed using $0.5 \mathrm{mmol}$ of the corresponding thioester. Purification by flash chromatography on silica gel (hexanes/EtOAc, 97:3) afforded the product as a clear oil (78 mg, 77\% yield). ${ }^{1} \mathrm{H}$ NMR $\left(500 \mathrm{MHz}, \mathrm{CDCl}_{3}\right) \delta 7.60(\mathrm{~d}, J=7.9 \mathrm{~Hz}, 1 \mathrm{H}), 7.48-7.36(\mathrm{~m}, 2 \mathrm{H}), 7.21(\mathrm{t}, J=7.9 \mathrm{~Hz}, 1 \mathrm{H}), 6.78(\mathrm{t}$, $J=57.0 \mathrm{~Hz}, 1 \mathrm{H}), 3.69(\mathrm{p}, J=6.9 \mathrm{~Hz}, 1 \mathrm{H}), 1.25(\mathrm{~d}, J=7.0 \mathrm{~Hz}, 6 \mathrm{H}) .{ }^{13} \mathrm{C} \mathrm{NMR}\left(176 \mathrm{MHz}, \mathrm{CDCl}_{3}\right) \delta$ 153.07, 136.93, 130.52, 126.51, 124.46, 121.38 (t, $J=275.2 \mathrm{~Hz}), 30.80,23.64 .{ }^{19} \mathrm{~F}$ NMR $(471$ $\left.\mathrm{MHz}, \mathrm{CDCl}_{3}\right) \delta-91.16(\mathrm{~d}, J=57.0 \mathrm{~Hz}, 2 \mathrm{~F})$. HRMS (EI) calcd. for $\mathrm{C}_{10} \mathrm{H}_{12} \mathrm{~F}_{2} \mathrm{~S}[\mathrm{M}+] \mathrm{m} / \mathrm{z} 202.0628$, found 202.0626 . 
<smiles>Cc1cc(C)c(SC(F)F)c([N+](=O)[O-])c1</smiles>

(Difluoromethyl)(mesityl)sulfane (20). The general decarbonylation procedure was followed using $0.5 \mathrm{mmol}$ of the corresponding thioester. Purification by flash chromatography on silica gel (hexanes/EtOAc, 100:0) afforded the product as a clear oil (37 mg, 37\% yield). ${ }^{1} \mathrm{H}$ NMR (500 $\left.\mathrm{MHz}, \mathrm{CDCl}_{3}\right) \delta 7.01(\mathrm{~s}, 2 \mathrm{H}), 6.66(\mathrm{t}, J=57.1 \mathrm{~Hz}, 1 \mathrm{H}), 2.52(\mathrm{~s}, 6 \mathrm{H}), 2.31(\mathrm{~s}, 3 \mathrm{H}) .{ }^{13} \mathrm{C}$ NMR $(176$ $\left.\mathrm{MHz}, \mathrm{CDCl}_{3}\right) \delta 144.66,140.38,129.40,121.61(\mathrm{t}, J=275.4 \mathrm{~Hz}), 121.39,22.24,21.06 .{ }^{19} \mathrm{~F} \mathrm{NMR}$ $\left(471 \mathrm{MHz}, \mathrm{CDCl}_{3}\right) \delta-90.71(\mathrm{~d}, J=57.1 \mathrm{~Hz}, 2 \mathrm{~F})$. HRMS (GC-APCl) calcd. for $\mathrm{C}_{10} \mathrm{H}_{12} \mathrm{~F}_{2} \mathrm{~S}[\mathrm{M}+\mathrm{H}]$ $\mathrm{m} / \mathrm{z} 203.0728$, found 203.0701.<smiles>FC(F)SCCc1ccccc1</smiles>

(Difluoromethyl)(phenethyl)sulfane (2p). The general decarbonylation procedure was followed using $0.5 \mathrm{mmol}$ of the corresponding thioester. Purification by flash chromatography on silica gel (hexanes/EtOAc, 97:3) afforded the product as a clear oil (72 mg, 77\% yield). ${ }^{1} \mathrm{H}$ NMR (500 MHz, $\left.\mathrm{CDCl}_{3}\right) \delta 7.34(\mathrm{t}, J=7.4 \mathrm{~Hz}, 2 \mathrm{H}), 7.30-7.25(\mathrm{~m}, 1 \mathrm{H}), 7.24(\mathrm{~d}, J=7.4 \mathrm{~Hz}, 2 \mathrm{H}), 6.80(\mathrm{t}, J=56.1 \mathrm{~Hz}$, 1H), 3.11-3.05 (m, 2H), 3.03-2.98 (m, 2H). ${ }^{13} \mathrm{C} \mathrm{NMR} \mathrm{(126} \mathrm{MHz,} \mathrm{CDCl} 3$ ) ठ 139.53, 128.62, 128.56, 126.73, $120.63(\mathrm{t}, J=272.9 \mathrm{~Hz}), 36.75,28.56 .{ }^{19} \mathrm{~F} \mathrm{NMR}\left(377 \mathrm{MHz}, \mathrm{CDCl}_{3}\right) \delta-93.19(\mathrm{~d}, J=56.1$ $\mathrm{Hz}, 2 \mathrm{~F}$ ). HRMS (GC-APCl) calcd. for $\mathrm{C}_{9} \mathrm{H}_{10} \mathrm{~F}_{2} \mathrm{~S}[\mathrm{M}+] \mathrm{m} / \mathrm{z} 188.0471$, found 188.0466 .

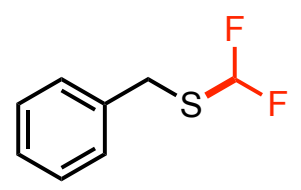

Benzyl(difluoromethyl)sulfane (2q). The general decarbonylation procedure was followed using $0.5 \mathrm{mmol}$ of the corresponding thioester. Purification by flash chromatography on silica gel (hexanes/EtOAc, 97:3) afforded the product as a clear oil ( $48 \mathrm{mg}, 55 \%$ yield). To demonstrate that the low yield was due to the volatility of the product, the general decarbonylation procedure was followed using $0.15 \mathrm{mmol}$ of the corresponding thioester, and the yield was determined by ${ }^{19} \mathrm{~F}$ NMR spectroscopy with 4 -fluorotoluene as the internal standard (97\% yield). ${ }^{1} \mathrm{H}$ NMR (500 $\mathrm{MHz}, \mathrm{CDCl}_{3}$ ) $\delta 7.42-7.32$ (multiple peaks, $5 \mathrm{H}$ ), $6.75\left(\mathrm{t}, J=56.6 \mathrm{~Hz}, 1 \mathrm{H}\right.$ ), $4.04(\mathrm{~s}, 2 \mathrm{H}) .{ }^{13} \mathrm{C}$ NMR $\left(126 \mathrm{MHz}, \mathrm{CDCl}_{3}\right) \delta 136.23,128.87,128.78,127.63,120.22(\mathrm{t}, J=272.9 \mathrm{~Hz}), 31.74 .{ }^{19} \mathrm{~F}$ NMR 
(377 MHz, $\left.\mathrm{CDCl}_{3}\right) \delta-94.78$ (d, J = 56.6 Hz, 2F). HRMS (EI) calcd. for $\mathrm{C}_{8} \mathrm{H}_{8} \mathrm{~F}_{2} \mathrm{~S}[\mathrm{M}+] \mathrm{m} / \mathrm{z} 174.0315$, found 174.0315 .

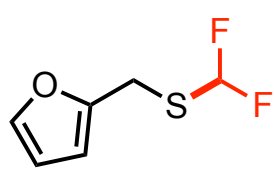

2-(((Difluoromethyl)thio)methyl)furan (2r). The general decarbonylation procedure was followed using $0.15 \mathrm{mmol}$ of the corresponding thioester, and the yield was determined by ${ }^{19} \mathrm{~F}$ NMR spectroscopy with 4-fluorotoluene as the internal standard ( $24 \%$ yield). ${ }^{19} \mathrm{~F}$ NMR shifts were consistent with formation of decarbonylated (difluoromethyl)thioether, ${ }^{19} \mathrm{~F} \mathrm{NMR}\left(377 \mathrm{~Hz}, \mathrm{CDCl}_{3}\right)$ $\delta-90.86 \mathrm{ppm}(\mathrm{d}, J=57.1 \mathrm{~Hz}, 2 \mathrm{~F})$. Due to the high volatility of the product, attempts at isolation were unsuccessful.<smiles>FC(F)SC1CCCCC1</smiles>

Cyclohexyl(difluoromethyl)sulfane (2s). The general decarbonylation procedure was followed using $0.5 \mathrm{mmol}$ of the corresponding thioester. Purification by flash chromatography on silica gel (hexanes/EtOAc, 97:3) afforded the product as a clear oil (38 mg, 43\% yield). To demonstrate that the low yield was due to the volatility of the product, the general decarbonylation procedure was followed using $0.15 \mathrm{mmol}$ of the corresponding thioester, and the yield was determined by ${ }^{19} \mathrm{~F}$ NMR spectroscopy with 4-fluorotoluene as the internal standard (99\% yield). ${ }^{1} \mathrm{H}$ NMR $\left({ }^{1} \mathrm{H}\right.$ $\operatorname{NMR}\left(500 \mathrm{MHz}, \mathrm{CDCl}_{3}\right) \delta 6.86(\mathrm{t}, J=56.7 \mathrm{~Hz}, 1 \mathrm{H}), 3.20$ (tt, $\left.J=10.6,3.8 \mathrm{~Hz}, 1 \mathrm{H}\right), 2.04(\mathrm{dd}, J=$ $13.4,4.1 \mathrm{~Hz}, 2 \mathrm{H}), 1.83-1.74(\mathrm{~m}, 2 \mathrm{H}), 1.66-1.59(\mathrm{~m}, 1 \mathrm{H}), 1.54-1.45(\mathrm{~m}, 2 \mathrm{H}), 1.45-1.34(\mathrm{~m}, 2 \mathrm{H})$, 1.35-1.23 (m, 1H). ${ }^{13} \mathrm{C}$ NMR (126 MHz, $\left.\mathrm{CDCl}_{3}\right) \delta 120.98(\mathrm{t}, \mathrm{J}=271.7 \mathrm{~Hz}), 41.66,34.44,25.83$, 25.36. ${ }^{19} \mathrm{~F}$ NMR $\left(377 \mathrm{MHz}, \mathrm{CDCl}_{3}\right) \delta-91.49$ (d, $\left.J=56.7 \mathrm{~Hz}, 2 \mathrm{~F}\right)$. HRMS (GC-APCl) calcd. for $\mathrm{C}_{7} \mathrm{H}_{12} \mathrm{~F}_{2} \mathrm{~S}[\mathrm{M}-\mathrm{F}]+\mathrm{m} / \mathrm{z}$ 147.0644, found 147.0638 .

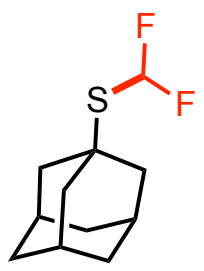

((3s,5s,7s)-Adamantan-1-yl)(difluoromethyl)sulfane (2t). The general decarbonylation procedure was followed using $0.5 \mathrm{mmol}$ of the corresponding thioester. Purification by flash 
chromatography on silica gel (hexanes/EtOAc, 97:3) afforded the product as a clear oil (77 mg, $71 \%$ yield). ${ }^{1} \mathrm{H}$ NMR (500 MHz, $\left.\mathrm{CDCl}_{3}\right) \delta 6.95$ (t, $\left.J=56.9 \mathrm{~Hz}, 1 \mathrm{H}\right), 2.10-2.05(\mathrm{br}, 3 \mathrm{H}), 2.02$ (d, J $=3.2 \mathrm{~Hz}, 6 \mathrm{H}), 1.72(\mathrm{br}, 6 \mathrm{H}) \cdot{ }^{13} \mathrm{C}$ NMR $\left(126 \mathrm{MHz}, \mathrm{CDCl}_{3}\right) \delta 120.37$ (t, J = 269.3 Hz), 48.29, 44.23, 35.94, 29.76. ${ }^{19} \mathrm{~F}$ NMR (471 MHz, $\left.\mathrm{CDCl}_{3}\right) \delta-90.16$ (d, $\left.J=56.9 \mathrm{~Hz}, 2 \mathrm{~F}\right)$. HRMS (EI) calcd. for $\mathrm{C}_{11} \mathrm{H}_{16} \mathrm{~F}_{2} \mathrm{~S}[\mathrm{M}+] \mathrm{m} / \mathrm{z} 218.0941$, found 218.0936 .

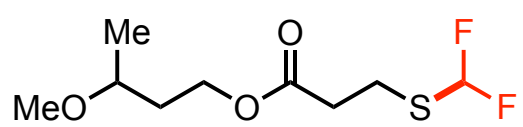

3-Methoxybutyl 3-((difluoromethyl)thio)propanoate (2u). The general decarbonylation procedure was followed using $0.5 \mathrm{mmol}$ of the corresponding thioester. Purification by flash chromatography on silica gel (hexanes/EtOAc, 60:40) afforded the product as a clear oil (56 mg, $46 \%$ yield). ${ }^{1} \mathrm{H}$ NMR $\left(500 \mathrm{MHz}, \mathrm{CDCl}_{3}\right) \delta 6.85(\mathrm{t}, J=56.0 \mathrm{~Hz}, 1 \mathrm{H}), 4.23(\mathrm{t}, J=7.0 \mathrm{~Hz}, 2 \mathrm{H}), 3.42$ (dqd, $J=7.7,6.2,4.6 \mathrm{~Hz}, 1 \mathrm{H}$ ), $3.33(\mathrm{~s}, 3 \mathrm{H}), 3.07$ (t, $J=7.1 \mathrm{~Hz}, 2 \mathrm{H}), 2.74(\mathrm{t}, J=7.1 \mathrm{~Hz}, 2 \mathrm{H}), 1.93-$ $1.70(\mathrm{~m}, 2 \mathrm{H}), 1.18(\mathrm{~d}, J=6.2 \mathrm{~Hz}, 3 \mathrm{H}) .{ }^{13} \mathrm{C}$ NMR $\left(176 \mathrm{MHz}, \mathrm{CDCl}_{3}\right) \delta 171.29,120.52(\mathrm{t}, J=273.0$ $\mathrm{Hz}), 73.58,61.98,56.11,35.58,35.47,22.13(\mathrm{t}, J=3.6 \mathrm{~Hz}), 19.03 .{ }^{19} \mathrm{~F} \mathrm{NMR}\left(377 \mathrm{MHz}, \mathrm{CDCl}_{3}\right) \delta$ $-93.17(\mathrm{~d}, \mathrm{~J}=56.0 \mathrm{~Hz}, 2 \mathrm{~F})$. HRMS (EI) calcd. for $\mathrm{C}_{9} \mathrm{H}_{16} \mathrm{~F}_{2} \mathrm{O}_{3} \mathrm{~S}[\mathrm{M}+] \mathrm{m} / \mathrm{z} 242.0788$, found 242.0793.

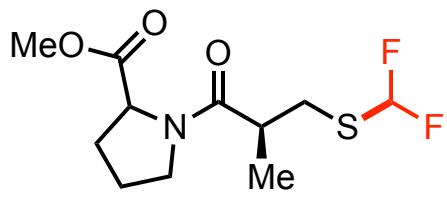

Methyl ((S)-3-((difluoromethyl)thio)-2-methylpropanoyl)prolinate (2v). The general decarbonylation procedure was followed using $0.3 \mathrm{mmol}$ of the corresponding thioester. Flash chromatography on silica gel (hexanes/EtOAc, 60:40) afforded a mixture of two diastereomer products ( $\mathrm{dr}=1.0: 0.14)$ as a clear oil ( $60 \mathrm{mg}, 71 \%$ yield). The diagnostic peaks of the chiral proton alpha to the ester and amide, as well as both $-\mathrm{CH}_{3}$ signals, were used to determine the diastereomeric ratio. Relative integrations assisted in determining the remaining signals for the major diastereomer. NMR (500 MHz, $\left.\mathrm{CDCl}_{3}\right) \delta 6.83(\mathrm{t}, J=56.3 \mathrm{~Hz}, 1 \mathrm{H}), 4.55(\mathrm{dd}, J=8.6,4.0 \mathrm{~Hz}$, 1H), $3.73(\mathrm{~s}, 3 \mathrm{H}), 3.65(\mathrm{t}, J=6.6 \mathrm{~Hz}, 2 \mathrm{H}), 3.11(\mathrm{dd}, J=13.8,9.1 \mathrm{~Hz}, 1 \mathrm{H}), 2.94(\mathrm{~h}, J=6.8 \mathrm{~Hz}, 1 \mathrm{H})$, $2.82(\mathrm{dd}, J=13.8,5.2 \mathrm{~Hz}, 1 \mathrm{H}), 2.27-2.17(\mathrm{~m}, 1 \mathrm{H}), 2.16-1.96(\mathrm{~m}, 3 \mathrm{H}), 1.26(\mathrm{~d}, J=6.8 \mathrm{~Hz}, 3 \mathrm{H})$ for the major diastereomer. ${ }^{13} \mathrm{C}$ NMR $\left(126 \mathrm{MHz} \mathrm{CDCl}_{3}\right) \delta 173.15,172.60,121.15$ (t, J = 272.5 Hz), $58.58,52.10,46.80,39.90,30.21,29.01,24.76,17.02$ for the major diastereomer. ${ }^{19} \mathrm{~F}$ NMR $(471$ $\mathrm{MHz}, \mathrm{CDCl}_{3}$ ) $\delta-90.93$ to-92.97 (multiple peaks, 2F). HRMS (GC-APCI) calcd. for $\mathrm{C}_{11} \mathrm{H}_{17} \mathrm{~F}_{2} \mathrm{NO}_{3} \mathrm{~S}$ $[\mathrm{M}+\mathrm{H}]+m / z 282.0997$, found 282.0983 . 


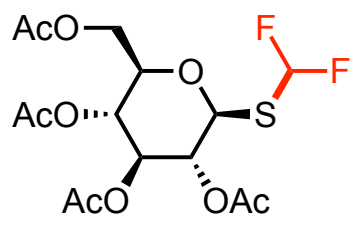

(2R,3R,4S,5R,6S)-2-(Acetoxymethyl)-6-((difluoromethyl)thio)tetrahydro-2H-pyran-3,4,5-

triyl triacetate $(2 \mathbf{w})$. The general decarbonylation procedure was followed using $0.5 \mathrm{mmol}$ of the corresponding thioester. Flash chromatography on silica gel (hexanes/EtOAc, 45:55) afforded the purified product as a white solid (62.6 mg, 47\% yield). mp 79.9-83.2 ${ }^{\circ} \mathrm{C}$. ${ }^{1} \mathrm{H}$ NMR $(700 \mathrm{MHz}$, $\left.\mathrm{CDCl}_{3}\right) \delta 7.25(\mathrm{t}, J=56.4 \mathrm{~Hz}, 1 \mathrm{H}), 5.54(\mathrm{t}, J=9.7 \mathrm{~Hz}, 1 \mathrm{H}), 5.37(\mathrm{dt}, J=31.6,10.0 \mathrm{~Hz}, 1 \mathrm{H}), 5.20$ (d, $J=10.2 \mathrm{~Hz}, 1 \mathrm{H}), 4.55$ (d, $J=12.2 \mathrm{~Hz}, 1 \mathrm{H}), 4.42$ (d, $J=12.5 \mathrm{~Hz}, 1 \mathrm{H}), 2.37$ (s, 3H), 2.35 (s, $3 \mathrm{H}), 2.32$ (s, 3H), 2.30 (s, 3H). ${ }^{13} \mathrm{C}$ NMR (176 MHz, $\left.\mathrm{CDCl}_{3}\right) \delta 170.79,170.24,169.51,169.49$, $119.08(\mathrm{t}, J=278.8 \mathrm{~Hz}), 80.09,76.52,73.72,70.00,68.09,61.98,20.78 .{ }^{19} \mathrm{~F} \mathrm{NMR}(376 \mathrm{MHz}$, $\left.\mathrm{CDCl}_{3}\right) \delta-95.92(\mathrm{dd}, J=248.0,56.4 \mathrm{~Hz}, 1 \mathrm{~F}),-99.22$ (dd, $\left.J=248.0,56.4 \mathrm{~Hz}, 1 \mathrm{~F}\right)$. HRMS (ESI) calcd. for $\mathrm{C}_{15} \mathrm{H}_{20} \mathrm{~F}_{2} \mathrm{O}_{9} \mathrm{~S}[\mathrm{M}+\mathrm{Na}] \mathrm{m} / \mathrm{z} 437.0694$, found 437.0688 .<smiles>COc1ccc(SCF)cc1</smiles>

(Fluoromethyl)(4-methoxyphenyl)sulfane (2x). The general decarbonylation procedure was followed using $0.3 \mathrm{mmol}$ of the corresponding thioester. Purification by flash chromatography on silica gel (hexanes/EtOAc, 99:1) afforded $2 x$ as a clear oil (41 mg, 71\% yield). ${ }^{1} \mathrm{H}$ NMR (500 MHz, $\left.\mathrm{CDCl}_{3}\right) \delta 6.85(\mathrm{t}, J=56.0 \mathrm{~Hz}, 1 \mathrm{H}), 4.23(\mathrm{t}, J=7.0 \mathrm{~Hz}, 2 \mathrm{H}), 3.42(\mathrm{dqd}, J=7.7,6.2,4.6 \mathrm{~Hz}, 1 \mathrm{H})$, $3.33(\mathrm{~s}, 3 \mathrm{H}), 3.07(\mathrm{t}, J=7.1 \mathrm{~Hz}, 2 \mathrm{H}), 2.74(\mathrm{t}, J=7.1 \mathrm{~Hz}, 2 \mathrm{H}), 1.93-1.70(\mathrm{~m}, 2 \mathrm{H}), 1.18(\mathrm{~d}, J=6.2$ $\mathrm{Hz}, 3 \mathrm{H}) .{ }^{13} \mathrm{C}$ NMR $\left(176 \mathrm{MHz}, \mathrm{CDCl}_{3}\right) \delta 171.29,120.52(\mathrm{t}, \mathrm{J}=273.0 \mathrm{~Hz}), 73.58,61.98,56.11$, 35.58, 35.47, $22.13(\mathrm{t}, \mathrm{J}=3.6 \mathrm{~Hz}), 19.03 .{ }^{19} \mathrm{~F} \mathrm{NMR}\left(377 \mathrm{MHz} \mathrm{CDCl}_{3}\right) \delta-93.17$ (t, $\mathrm{J}=56.0 \mathrm{~Hz}$, 1F). HRMS (EI) calcd. for $\mathrm{C}_{8} \mathrm{H}_{9} \mathrm{FOS}[\mathrm{M}+] \mathrm{m} / \mathrm{z} 172.0358$, found 172.0361 .

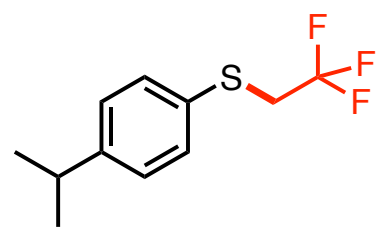

(4-Isopropylphenyl)(2,2,2-trifluoroethyl)sulfane (2y). The general decarbonylation procedure was followed using $0.3 \mathrm{mmol}$ of the corresponding thioester. Purification by flash chromatography 
on silica gel (hexanes/EtOAc, 99:1) afforded $2 y$ as a clear oil $\left(57 \mathrm{mg}, 81 \%\right.$ yield). ${ }^{1} \mathrm{H}$ NMR (500 $\left.\mathrm{MHz}, \mathrm{CDCl}_{3}\right) \delta 7.43$ (d, $\left.J=8.5 \mathrm{~Hz}, 1 \mathrm{H}\right), 7.20(\mathrm{~d}, J=8.3 \mathrm{~Hz}, 1 \mathrm{H}$ ), 3.40 (q, $J=9.8 \mathrm{~Hz}, 2 \mathrm{H}), 2.90$ (hept, $J=6.9 \mathrm{~Hz}, 1 \mathrm{H}), 1.24(\mathrm{~d}, J=7.0 \mathrm{~Hz}, 3 \mathrm{H}) .{ }^{13} \mathrm{C} \mathrm{NMR}\left(176 \mathrm{MHz}, \mathrm{CDCl}_{3}\right) \delta 149.53,132.72$, 130.61, 127.64, 125.66 (q, $J=276.5 \mathrm{~Hz}), 38.85$ (q, $J=32.4 \mathrm{~Hz}), 34.01,24.04 .{ }^{19} \mathrm{~F}$ NMR $(377$ $\left.\mathrm{MHz}, \mathrm{CDCl}_{3}\right) \delta-66.39\left(\mathrm{t}, \mathrm{J}=9.7 \mathrm{~Hz}, 3 \mathrm{~F}\right.$ ). HRMS (El) calcd. for $\mathrm{C}_{11} \mathrm{H}_{13} \mathrm{~F}_{3} \mathrm{~S}[\mathrm{M}+] \mathrm{m} / \mathrm{z} 234.0690$, found 234.0691 .<smiles>COc1ccc(SC(F)C(F)(F)F)cc1</smiles>

(4-Methoxyphenyl)(1,2,2,2-tetrafluoroethyl)sulfane (2z). The general decarbonylation procedure was followed using $0.5 \mathrm{mmol}$ of the corresponding thioester. Purification by flash chromatography on silica gel (hexanes/EtOAc, 99:1) afforded $\mathbf{2 z}$ as a light yellow oil (105 $\mathrm{mg}$, $88 \%$ yield). ${ }^{1} \mathrm{H}$ NMR (500 MHz, $\mathrm{CDCl}_{3}$ ) $\delta 7.54$ (d, $J=7.9 \mathrm{~Hz}, 2 \mathrm{H}$ ), $6.92(\mathrm{~d}, J=7.9 \mathrm{~Hz}, 2 \mathrm{H}$ ), 5.72 (dq, $J=50.1,5.8 \mathrm{~Hz}, 1 \mathrm{H}$ ), $3.84(\mathrm{~s}, 2 \mathrm{H}) .{ }^{13} \mathrm{C}$ NMR $\left(126 \mathrm{MHz}, \mathrm{CDCl}_{3}\right) \delta 161.13,136.43,121.42$ (dd, $J=281.4,31.0 \mathrm{~Hz}), 119.27,115.05,97.43(\mathrm{dq}, J=231.6,36.8,36.4 \mathrm{~Hz}), 55.39 .{ }^{19} \mathrm{~F}$ NMR $(376$ $\mathrm{MHz}, \mathrm{CDCl}_{3}$ ) $\delta-76.38$ (dd, $J=16.1,5.8 \mathrm{~Hz}$ ), -166.99 (dq, $J=50.1,16.1 \mathrm{~Hz}$ ). HRMS (El) calcd. for $\mathrm{C}_{9} \mathrm{H}_{8} \mathrm{~F}_{4} \mathrm{OS}[\mathrm{M}-\mathrm{F}] \mathrm{m} / \mathrm{z} 221.0332$, found 221.0247 .

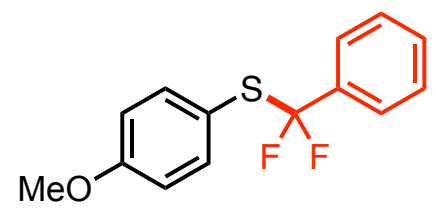

(difluoro(phenyl)methyl)(4-methoxyphenyl)sulfane (2aa). The general decarbonylation procedure was followed using $0.5 \mathrm{mmol}$ of the corresponding thioester. Loading of $\mathrm{Ni}(\operatorname{cod})_{2}$ and bidentate phosphine ligand was increased to $20 \mathrm{~mol} \%$ and $24 \mathrm{~mol} \%$, respectively. Xantphos was used instead of dppf. Purification by flash chromatography on silica gel $(0 \%-5 \% \mathbf{A} /$ hexanes; $\mathrm{A}=$ $15: 85 \mathrm{CHCl}_{3}: \mathrm{Et}_{2} \mathrm{O}$ ) afforded the product as a white solid $\left(76.4 \mathrm{mg}, 57 \%\right.$ yield). ${ }^{1} \mathrm{H} \mathrm{NMR}(500 \mathrm{MHz}$, $\left.\mathrm{CDCl}_{3}\right) \delta 7.57(\mathrm{~d}, J=6.8 \mathrm{~Hz}, 2 \mathrm{H}$ ), $7.54(\mathrm{~d}, J=8.6 \mathrm{~Hz}, 2 \mathrm{H}$ ), 7.40-7.47 (multiple peaks, $3 \mathrm{H}$ ), 6.91 $(\mathrm{d}, J=8.6 \mathrm{~Hz}, 2 \mathrm{H}), 3.84(\mathrm{~s}, 3 \mathrm{H}) .{ }^{13} \mathrm{C}$ NMR $\left(126 \mathrm{MHz}, \mathrm{CDCl}_{3}\right) \delta 161.18,138.24,130.44,128.27$, 127.50, $125.37(\mathrm{t}, J=4.5 \mathrm{~Hz}), 118.05,114.51,55.35 .{ }^{19} \mathrm{~F} \mathrm{NMR}\left(376 \mathrm{MHz}, \mathrm{CDCl}_{3}\right) \delta-72.44$. HRMS (GC-APCl) calcd. for $\mathrm{C}_{14} \mathrm{H}_{12} \mathrm{~F}_{2} \mathrm{OS}[\mathrm{M}+] \mathrm{m} / \mathrm{z} 266.0571$, found 266.0570 . 


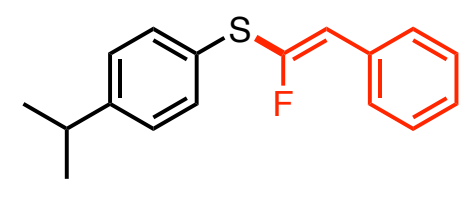

(1-Fluoro-2-phenylvinyl)(4-isopropylphenyl)sulfane (2ab). The general decarbonylation procedure was followed using $0.3 \mathrm{mmol}$ of the corresponding thioester. Purification by flash chromatography on silica gel (hexanes/EtOAc, 99:1) afforded 2ab as a thick oil (72 mg, 88\% yield). ${ }^{1} \mathrm{H}$ NMR (500 MHz, $\mathrm{CDCl}_{3}$ ) $\delta$ 7.56-7.49 (multiple peaks, $2 \mathrm{H}$ ), 7.47-7.40 (multiple peaks, 2H), 7.38-7.32 (multiple peaks, 2H), 7.31-7.25 (m, 1H), 7.24-7.19 (multiple peaks, 2H), 6.26 (d, $J=32.3 \mathrm{~Hz}, 1 \mathrm{H}), 2.90$ (hept, $J=6.9 \mathrm{~Hz}, 1 \mathrm{H}), 1.25(\mathrm{~d}, J=6.9 \mathrm{~Hz}, 6 \mathrm{H}) .{ }^{13} \mathrm{C} \mathrm{NMR}\left(176 \mathrm{MHz}, \mathrm{CDCl}_{3}\right)$ $\delta$ 153.79, 152.03, 148.90, 133.05 (d, $J=5.6 \mathrm{~Hz}), 130.55,128.77$ (d, $J=7.9 \mathrm{~Hz}), 128.58,127.99$ (d, $J=2.4 \mathrm{~Hz}), 127.49,116.93(\mathrm{~d}, J=12.8 \mathrm{~Hz}), 33.80,23.87 .{ }^{19} \mathrm{~F} \mathrm{NMR}\left(471 \mathrm{MHz}, \mathrm{CDCl}_{3}\right) \delta-$ $86.81\left(\mathrm{~d}, \mathrm{~J}=32.3 \mathrm{~Hz}, 1 \mathrm{~F}\right.$ ). HRMS (EI) calcd. for $\mathrm{C}_{17} \mathrm{H}_{17} \mathrm{FS}[\mathrm{M}+] \mathrm{m} / \mathrm{z} 272.1035$, found 272.1038 . 


\section{Catalyst screening for thiol trifluoromethylation}

\begin{tabular}{|c|c|c|c|}
\hline entry & {$[\mathrm{M}]$} & ligand & $\%$ yield $b$ \\
\hline 1 & $\mathrm{Ni}(\operatorname{cod})_{2}$ & dppf & 0 \\
\hline 2 & $\mathrm{Ni}(\operatorname{cod})_{2}$ & dpePhos & 0 \\
\hline 3 & $\mathrm{Ni}(\operatorname{cod})_{2}$ & Xantphos & 0 \\
\hline 4 & $\mathrm{Pd}(\mathrm{dba})_{2}$ & dppf & 0 \\
\hline 5 & $\mathrm{Pd}(\mathrm{dba})_{2}$ & dpePhos & 0 \\
\hline 6 & $\mathrm{Pd}(\mathrm{dba})_{2}$ & Xantphos & 0 \\
\hline 7 & $\mathrm{Pd}(\mathrm{dba})_{2}$ & $\mathrm{P}(\mathrm{Ad}) \mathrm{Bn}_{2}$ & 0 \\
\hline 8 & $\mathrm{Pd}(\mathrm{dba})_{2}$ & $\mathrm{P}(\mathrm{Ad})_{2} \mathrm{Bn}$ & 0 \\
\hline
\end{tabular}

${ }^{a} 24 \mathrm{~mol} \%$ used, ${ }^{b}$ Yields determined by ${ }^{19} \mathrm{~F}$ NMR

General procedure for optimizing the catalytic decarbonylation of difluoromethyl thiophenol ester 1a. Ni(cod) $)_{2}\left(4.1 \mathrm{mg}, 0.015 \mathrm{mmol}, 0.1\right.$ equiv) or $\mathrm{Pd}(\mathrm{dba})_{2}(13.7 \mathrm{mg}, 0.015 \mathrm{mmol}$, 0.1 equiv) and the appropriate phosphine ligand $(0.036 \mathrm{mmol}, 0.24$ equiv for monodentate ligands; $0.018 \mathrm{mmol}, 0.12$ equiv for bidentate ligands) were dissolved in $0.3 \mathrm{~mL}$ of solvent. The solution was stirred at room temperature for $15 \mathrm{~min}$ at which point the catalyst solution was transferred to $S$-phenyl 2,2,2,-trifluoroethanethiolate $(30.9 \mathrm{mg}, 0.15 \mathrm{mmol}, 1.0$ equiv) in a tall 10 $\mathrm{mL}$ vial. The vial was sealed with a Teflon-lined screw cap, brought out of the glovebox, and stirred at $130{ }^{\circ} \mathrm{C}$. After $4 \mathrm{~h}$ of heating, the reaction mixture was allowed to cool to room temperature. A stock solution of 4-fluorotoluene was prepared ( $0.5 \mathrm{M}$ in toluene) and added to the cooled reaction mixture $(0.3 \mathrm{~mL}, 0.15 \mathrm{mmol}, 1$ equiv). A sample of the crude reaction mixture with internal standard was removed for NMR analysis. Formation of $\mathrm{PhSCF}_{3}$ could not be observed by ${ }^{19} \mathrm{~F}$ NMR spectroscopy. 


\section{Stoichiometric decarbonylation of trifluoromethylthioesters}

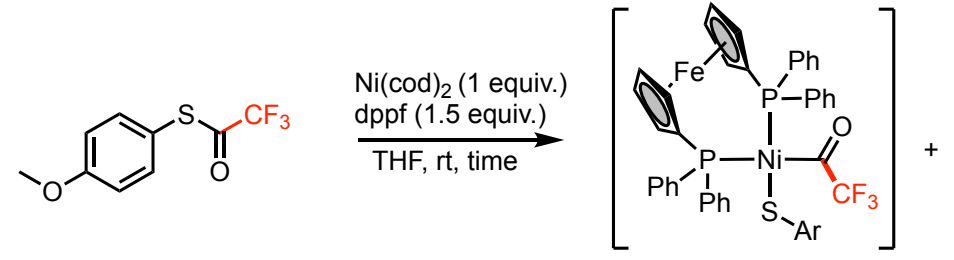

A

$15 \mathrm{~min}$

$3 \mathrm{~h}$

$17 \%$

$8 \%$

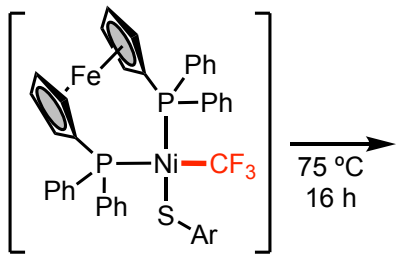

B

$0 \%$

$13 \%$

After 15 min @ rt
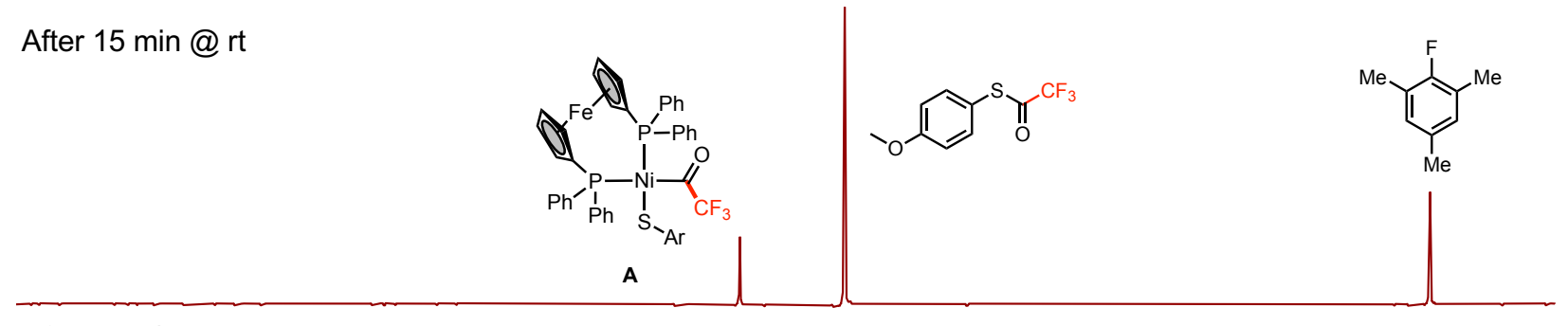

decomposition; no $\mathrm{ArSCF}_{3}$ observed

After3 h @ rt

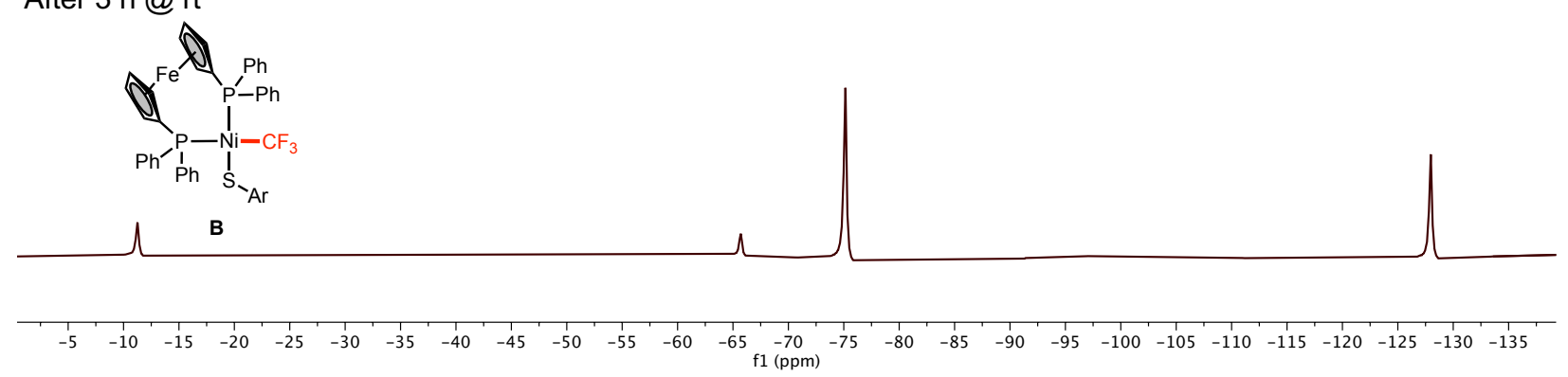

1 B

Figure S5. ${ }^{19} \mathrm{~F}$ NMR spectral data.

Stoichiometric decarbonylation of trifluoromethylthioesters. $\mathrm{Ni}(\operatorname{cod})_{2}(20.6 \mathrm{mg}, 0.075 \mathrm{mmol}$, 1.5 equiv) and dppf (30.3 mg, $0.075 \mathrm{mmol}, 1.5$ equiv) were dissolved in $0.5 \mathrm{~mL}$ of toluene. The solution was stirred at room temperature for $15 \mathrm{~min}$, at which point it was transferred to a preweighed mixture of $S$-(4-methoxyphenyl) 2,2,2,-trifluoroethanethiolate (11.8 mg, $0.05 \mathrm{mmol}, 1.0$ equiv) and the internal standard 2-fluoromesitylene $(0.05 \mathrm{mmol}, 1.0$ equiv). After mixing, the solution was transferred to a screw cap NMR tube and sealed with a Teflon-lined cap. The reaction mixture kept at room temperature and ${ }^{19} \mathrm{~F}$ NMR spectrum was recorded (Figure S5). 
XII. Heteroaromatic thiols and other failed substrates<smiles>O=C(OC(=O)C(F)F)C(F)F</smiles><smiles>[R]SC(=O)C(F)F</smiles><smiles>[R]SC(F)F</smiles>

A.<smiles>O=C(Sc1ccccn1)C(F)F</smiles><smiles>O=C(Sc1ccncc1)C(F)F</smiles><smiles>O=C(Sc1ncccn1)C(F)F</smiles><smiles>O=C(Sc1ccc2ccc(C(F)(F)F)cc2n1)C(F)F</smiles>

B.<smiles>O=C(Sc1cc2ccccc2o1)C(F)F</smiles><smiles>Cc1occc1SC(=O)C(F)F</smiles><smiles>COC(=O)c1ccccc1SC(=O)C(F)F</smiles><smiles>CC(SC(=O)C(F)F)c1ccccc1</smiles>

Figure S6. (a) Attempts at synthesis of these heteroaromatic substrates resulted in $\mathrm{N}$-acylation. (b) No decarbonylation observed after $20 \mathrm{~h}$ at $130^{\circ} \mathrm{C}$.

\section{References}

(1) Ferguson, D. M.; Bour, J. R.; Canty, A. J.; Kampf, J. W.; Sanford, M. S. Aryl-CF 3 Coupling from Phosphinoferrocene-Ligated Palladium(II) Complexes. Organometallics. 2019, 38, 519-526.

(2) Reina, A.; Krachko, T.; Onida, K.; Bouyssi, D.; Jeanneau, E.; Monteiro, N.; Amgoune, A. Development and Mechanistic Investigations of a Base-Free Suzuki-Miyaura Cross-Coupling of $\alpha, \alpha$-Difluoroacetamides via C-N Bond Cleavage. ACS Catal. 2020. 10, 3, 2189-2197.

(3) Mehta, V. P.; Greaney, M. F. S-, N-, and Se-Difluoromethylation Using Sodium, Chlorodifluoroacetate. Org. Lett. 2013, 15, 5036-5039. 
XIV. ${ }^{1} \mathrm{H},{ }^{13} \mathrm{C}$, and ${ }^{19} \mathrm{~F}$ NMR spectral data<smiles>O=C(Sc1ccccc1)C(F)F</smiles>

$1 a$

${ }^{1} \mathrm{H}$ NMR

ํำ웜ำ

तN

\।

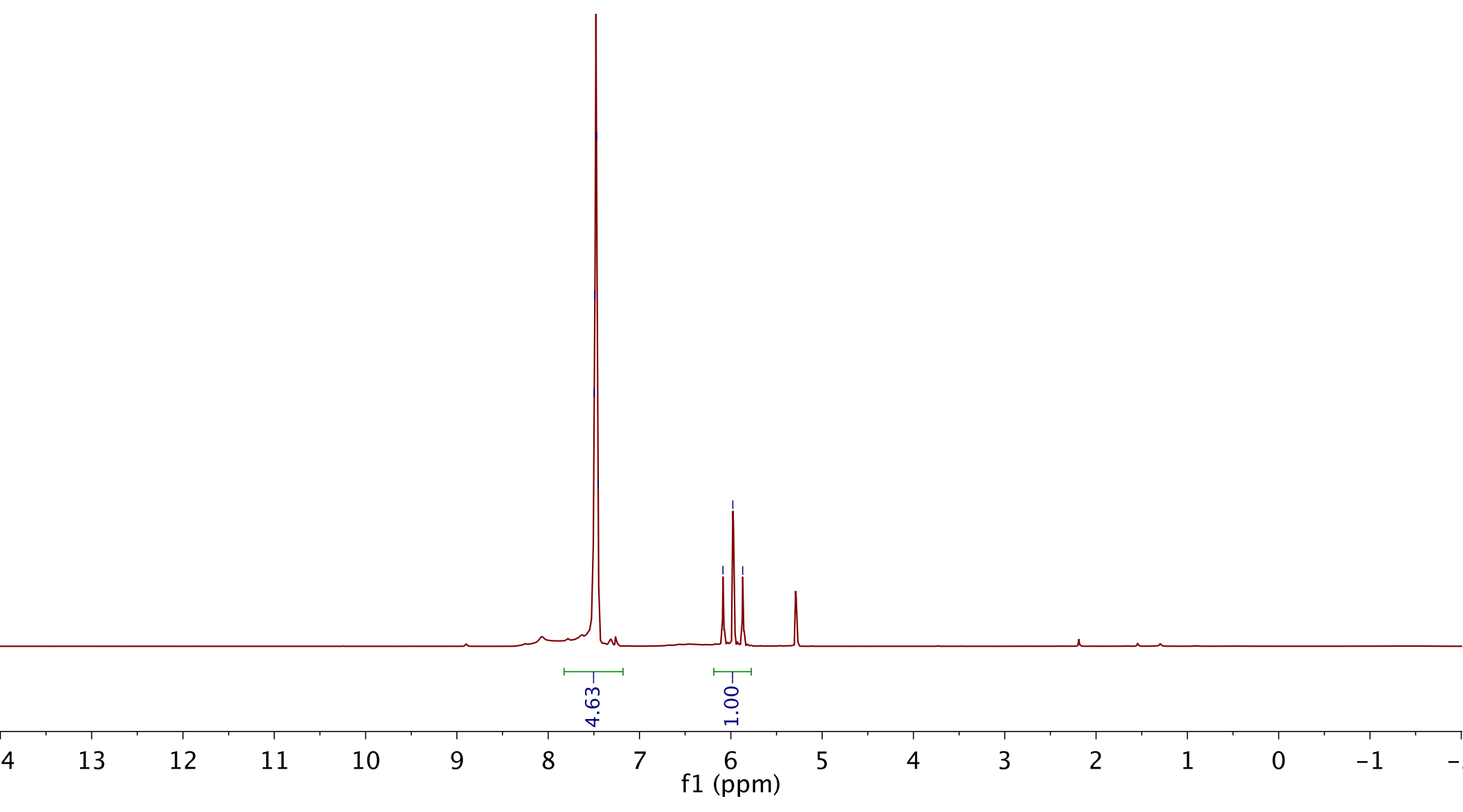


<smiles>O=C(Sc1ccccc1)C(F)F</smiles>

$1 a$

${ }^{13} \mathrm{C}$ NMR

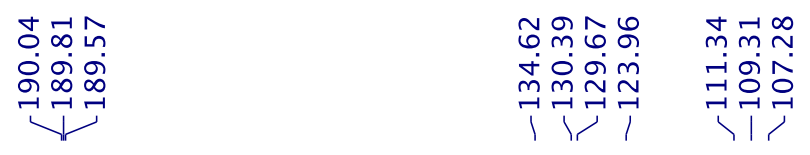

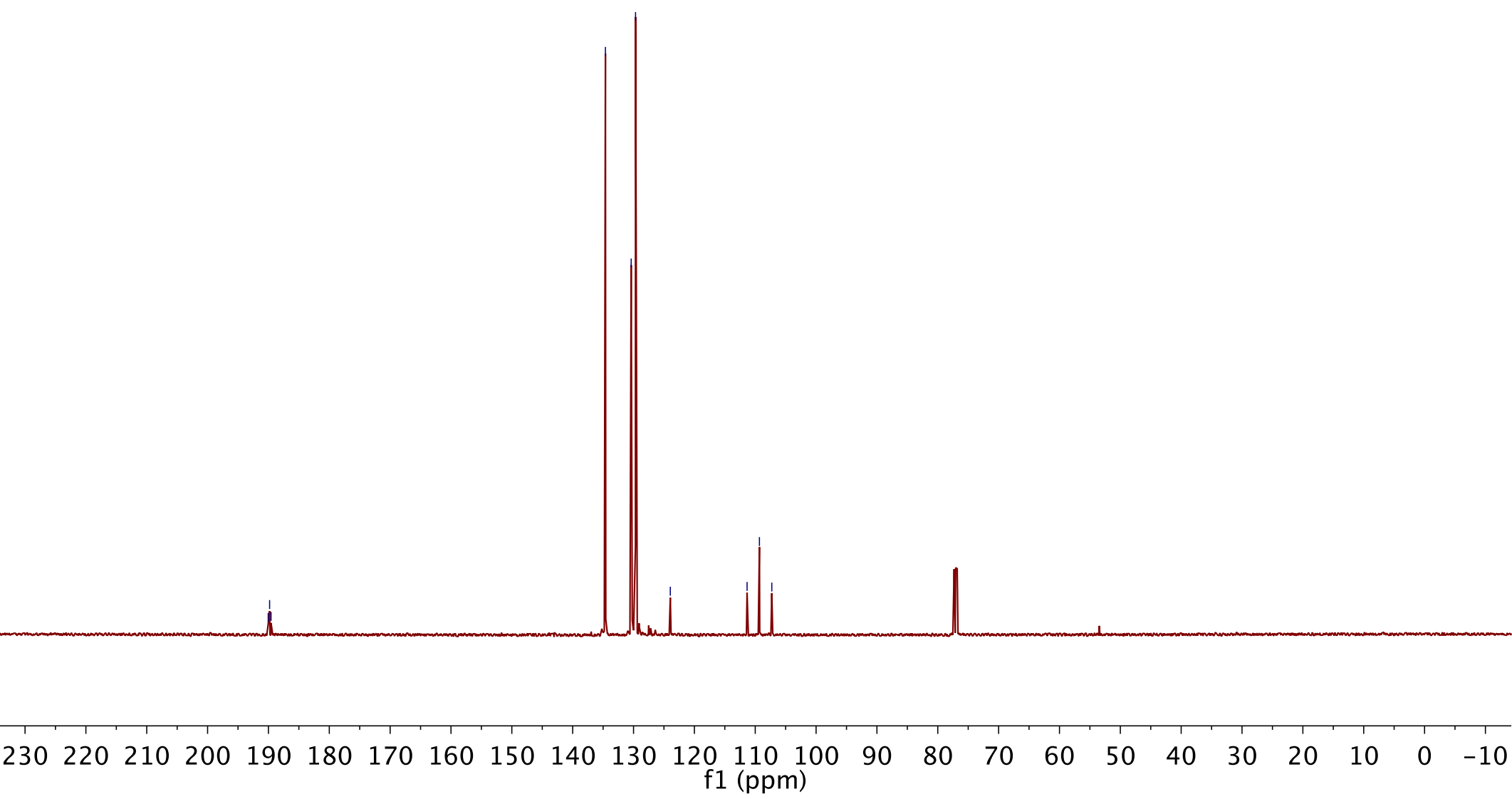




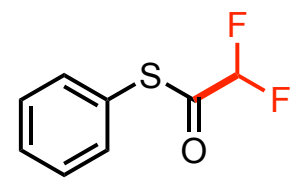

$1 a$

${ }^{19}$ F NMR
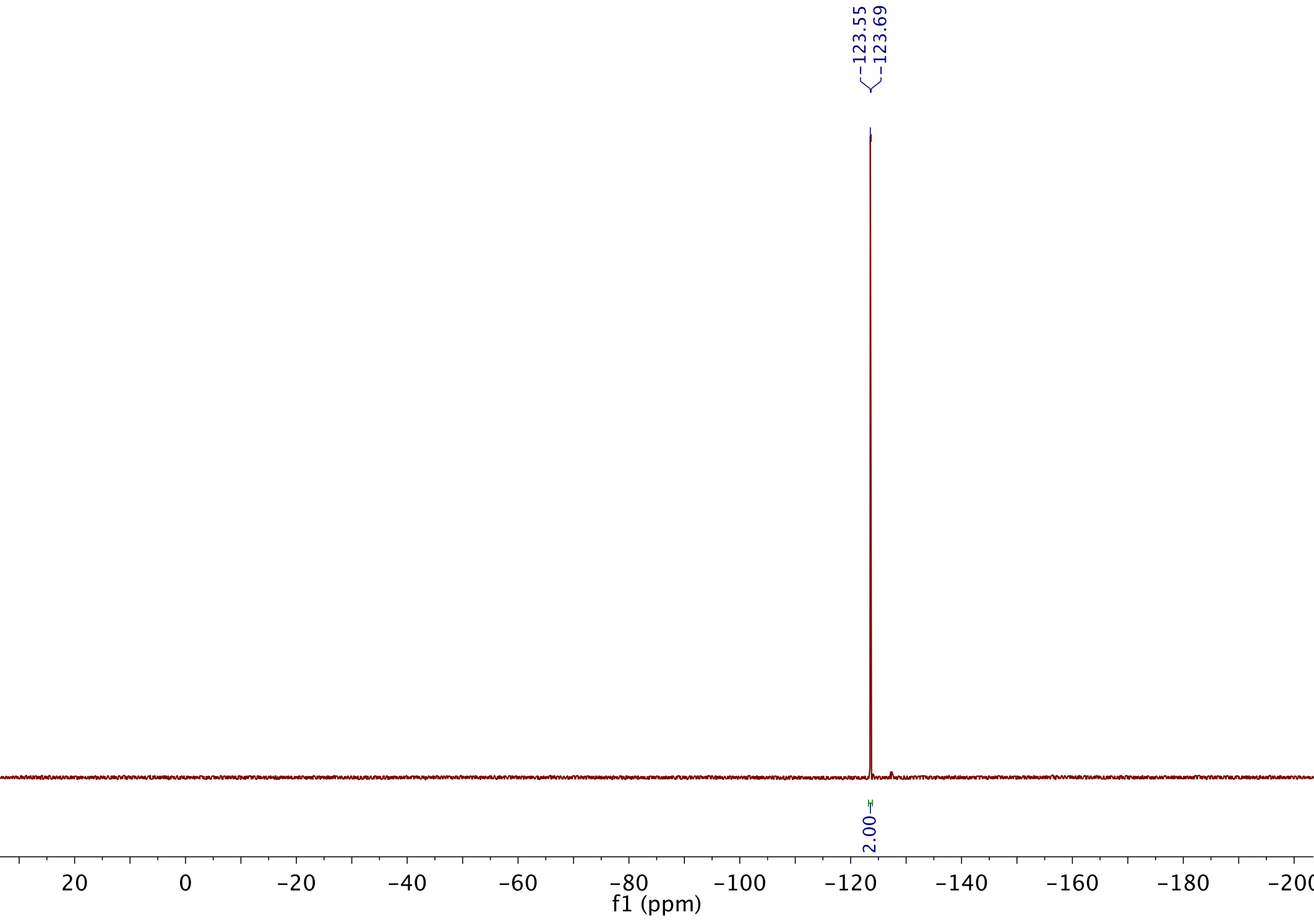
<smiles>COc1ccc(SC(=O)C(F)F)cc1</smiles>

$1 \mathrm{~b}$

${ }^{1} \mathrm{H}$ NMR

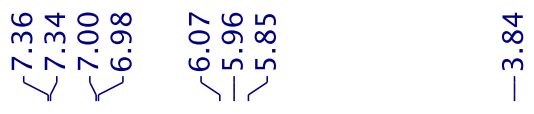

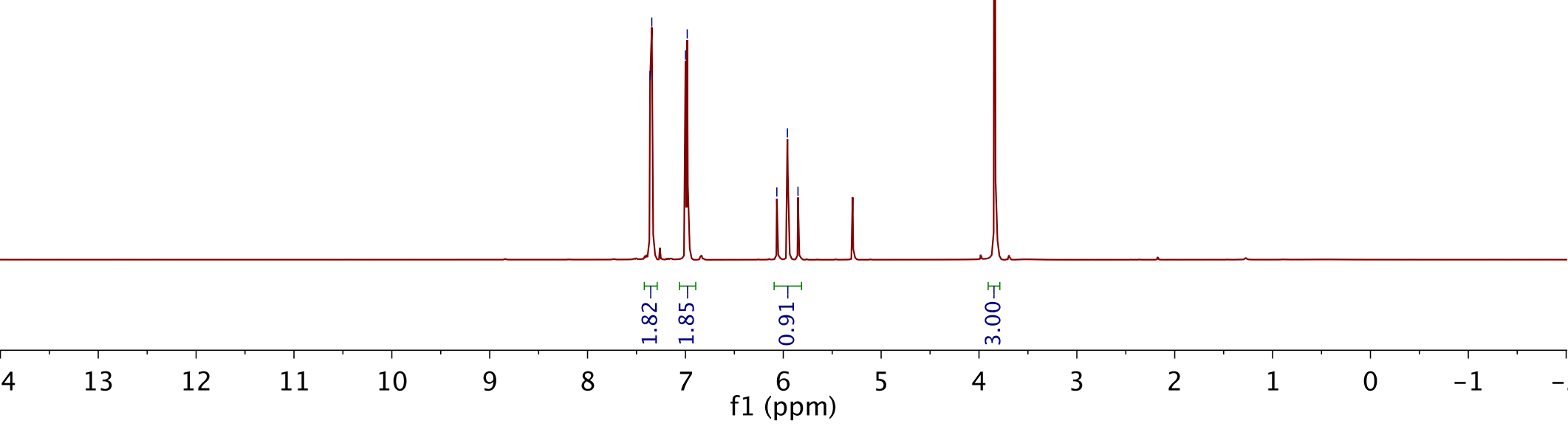


<smiles>COc1ccc(SC(=O)C(F)F)cc1</smiles>

$1 b$
${ }^{1}$ NMR

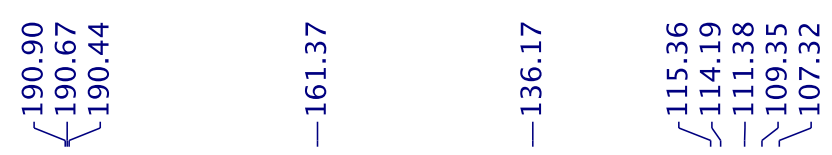

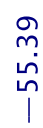

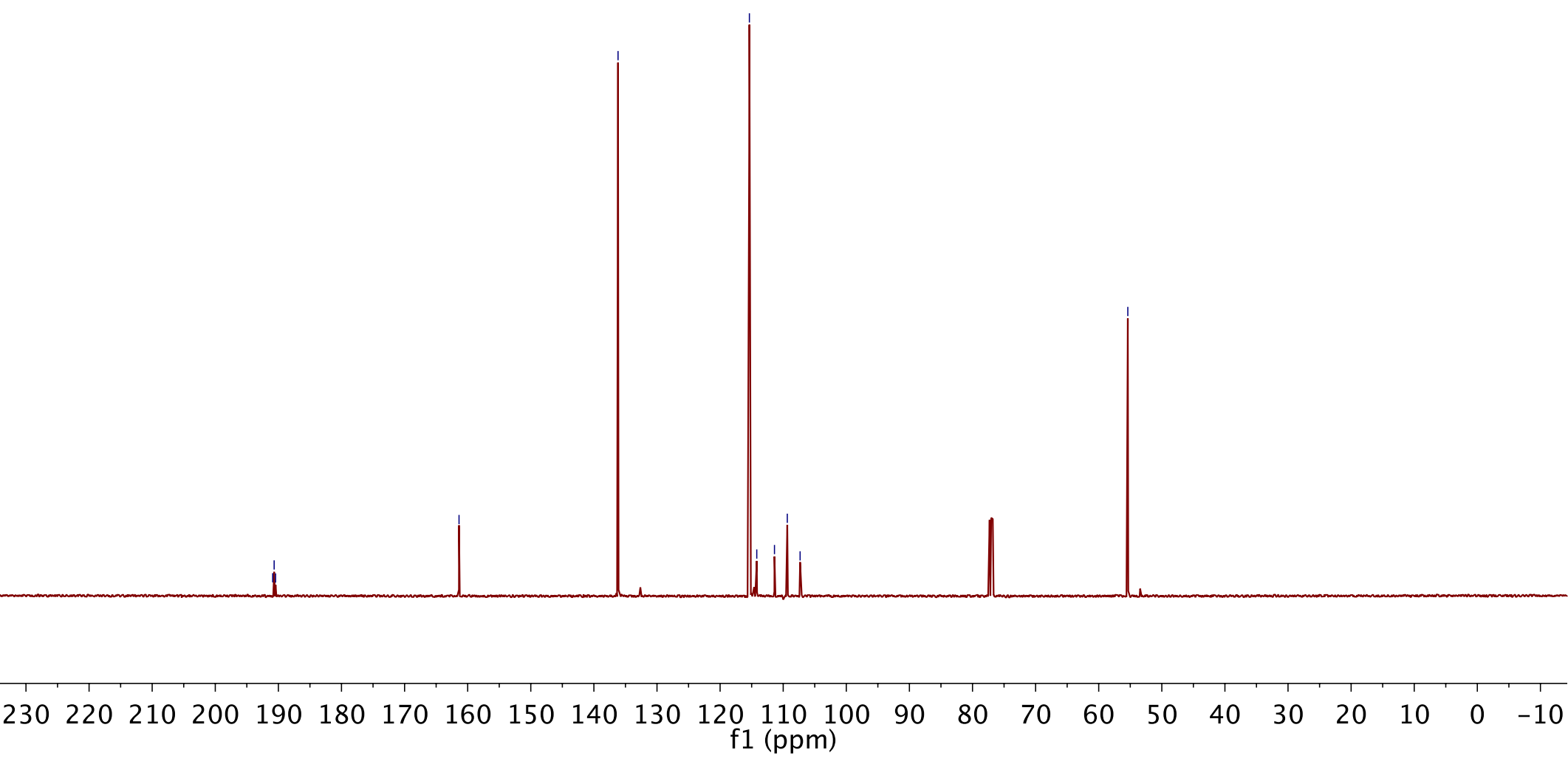


<smiles>COc1ccc(SC(=O)C(F)F)cc1</smiles>

$1 b$

${ }^{19} \mathrm{~F}$ NMR

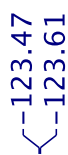

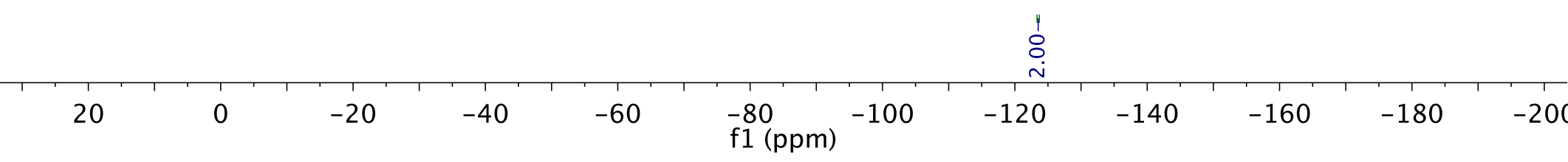




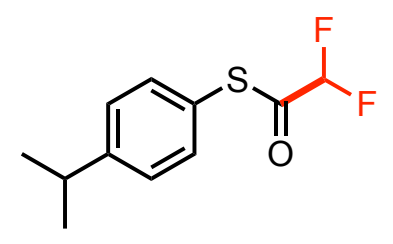

1c

${ }^{1} \mathrm{H}$ NMR

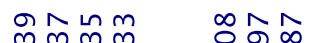

NiñN

에붕

mกำ

ָָ

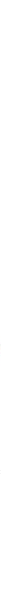


<smiles>CC(C)c1ccc(SC(=O)C(F)F)cc1</smiles>

$1 \mathrm{c}$

${ }^{13} \mathrm{C}$ NMR

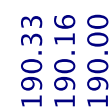

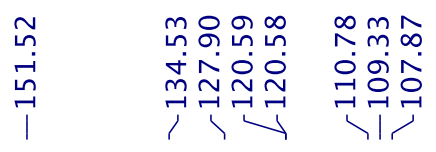

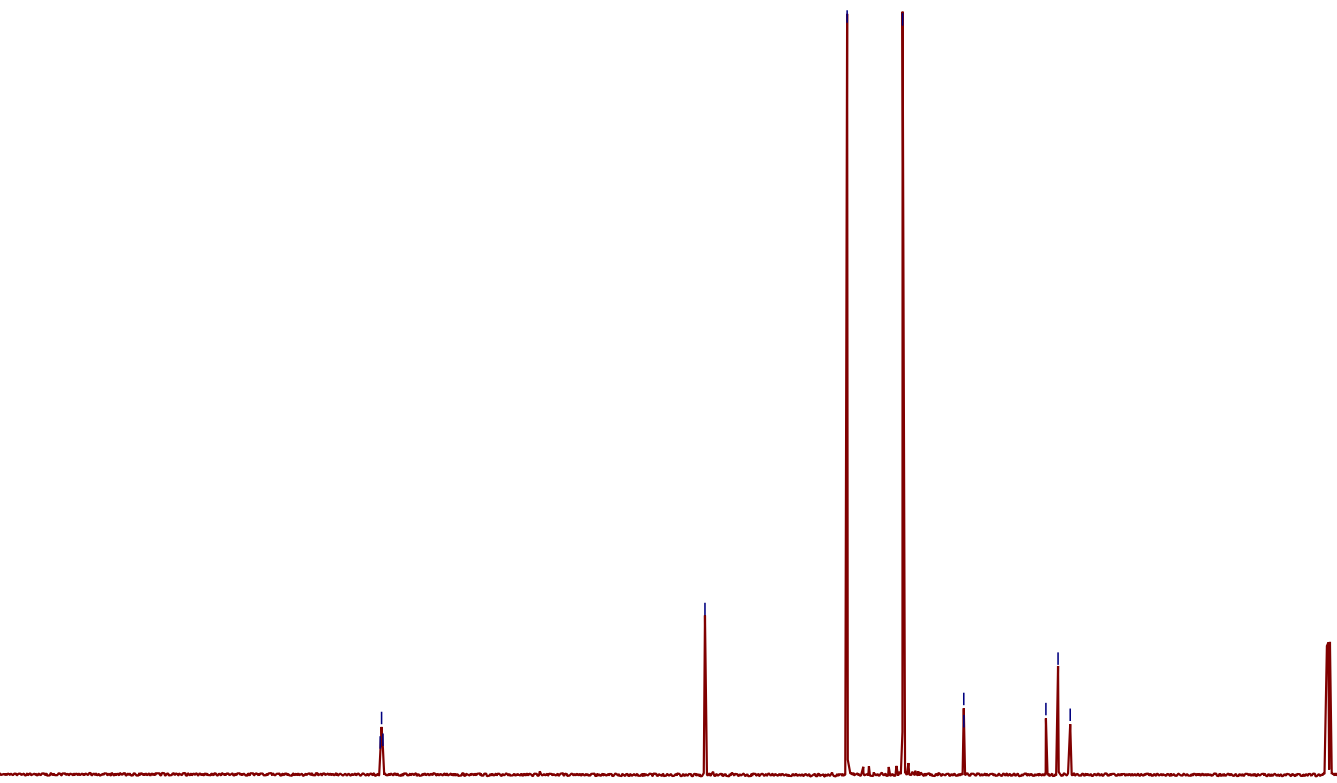

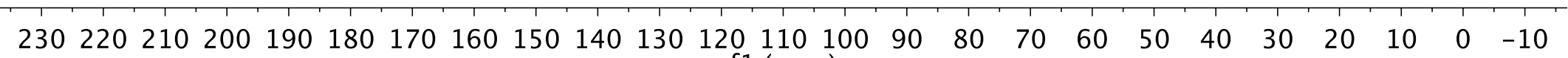
f1 (ppm) 


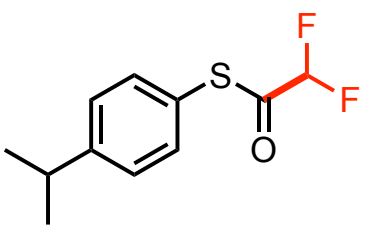

$1 \mathrm{c}$

${ }^{19} \mathrm{~F}$ NMR

ํㅗㄴ

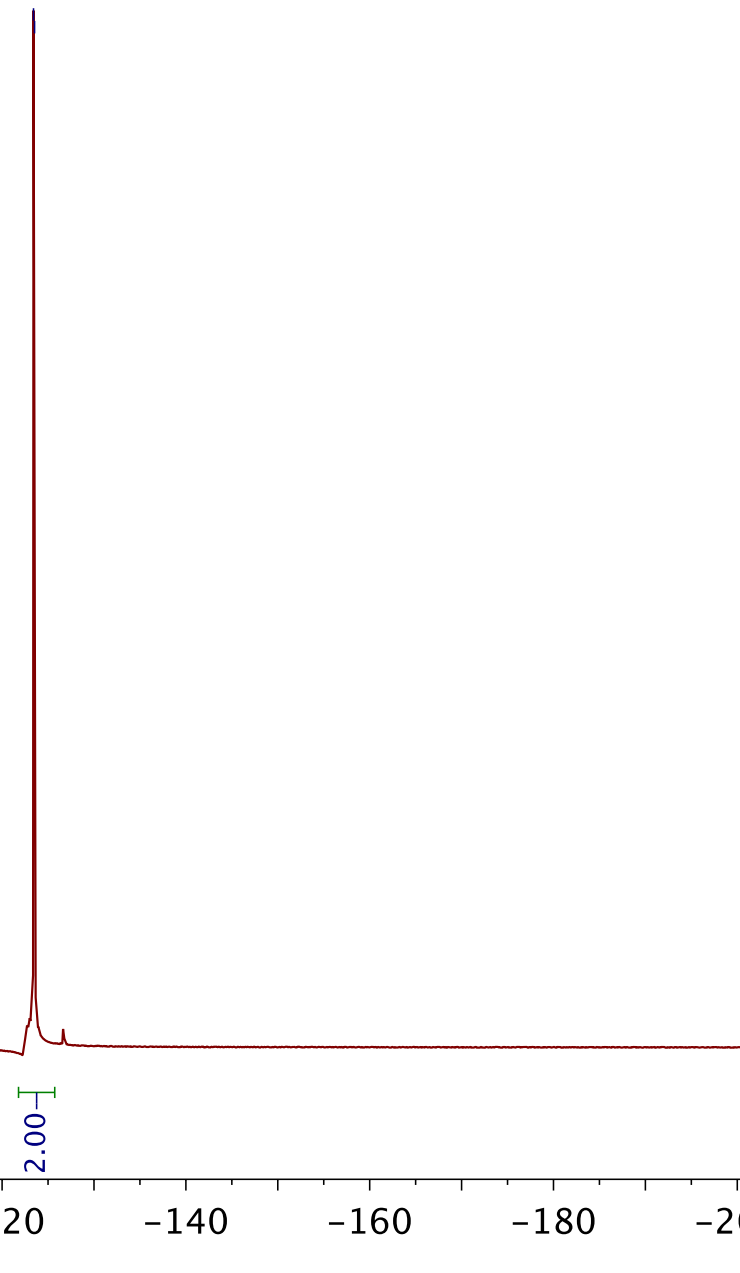




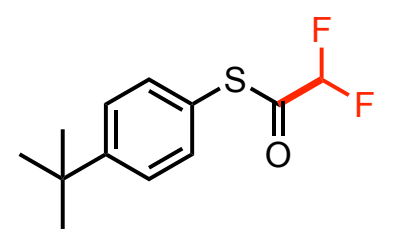

1d

${ }^{1} \mathrm{H}$ NMR

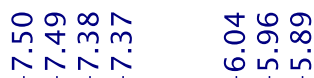

$\stackrel{\stackrel{n}{m}}{i}$

㖉

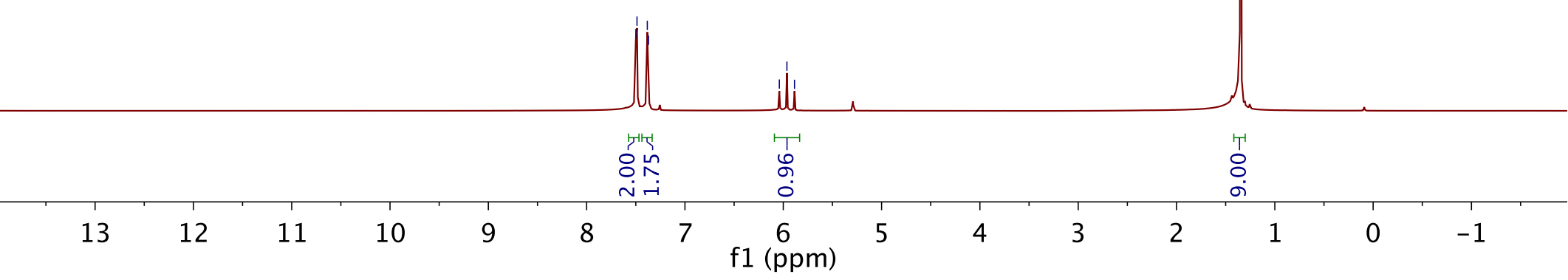




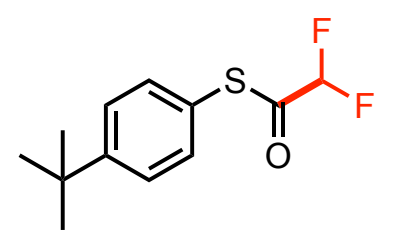

1d

${ }^{13} \mathrm{C}$ NMR
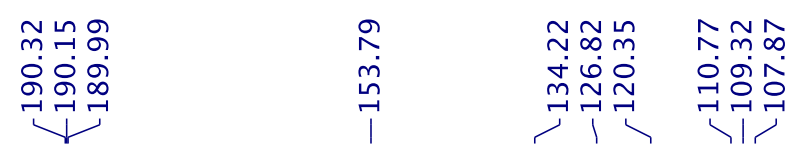

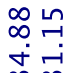

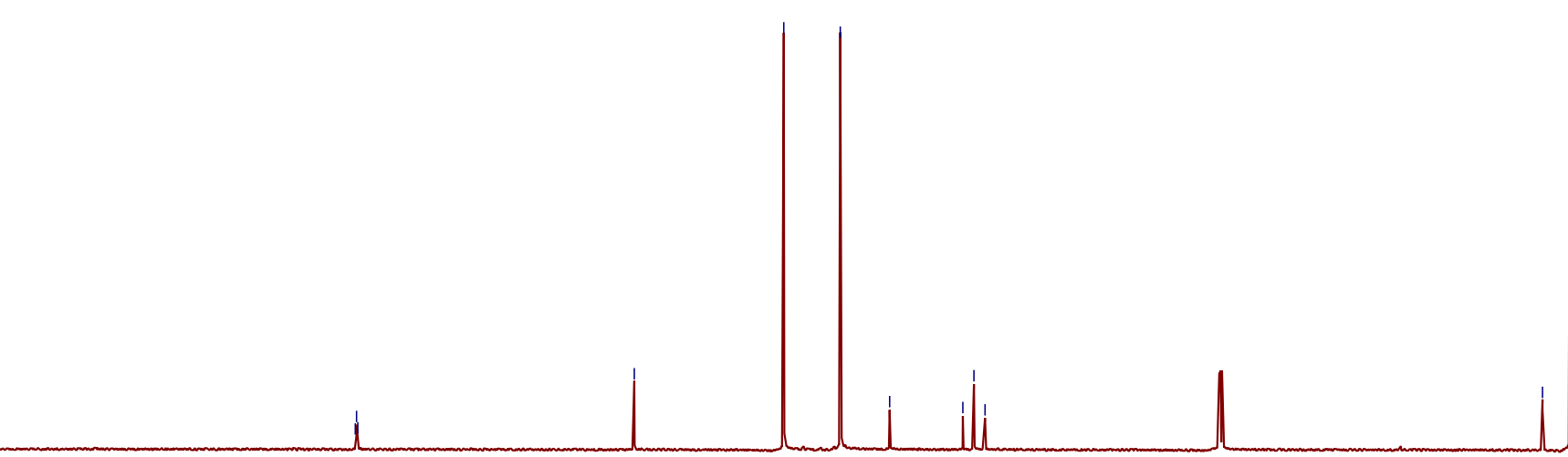

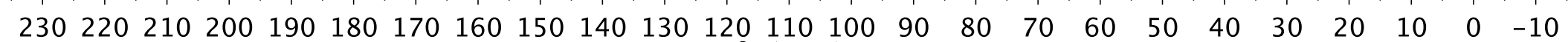 f1 (ppm)}


<smiles>CC(C)(C)c1ccc(SC(=O)C(F)F)cc1</smiles>

1d

${ }^{19} \mathrm{~F}$ NMR

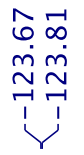

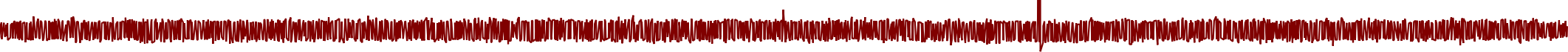

\begin{tabular}{|c|c|c|c|c|c|c|c|c|c|c|}
\hline 20 & 0 & -20 & -40 & -60 & $\begin{array}{l}-80 \\
\mathrm{f1}(\mathrm{ppm})\end{array}$ & -100 & -120 & -140 & -160 & -180 \\
\hline
\end{tabular}


<smiles>CN(C)c1ccc(SC(=O)C(F)F)cc1</smiles>

$1 e$

${ }^{1} \mathrm{H}$ NMR

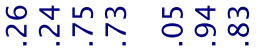

N

$\checkmark v>1$

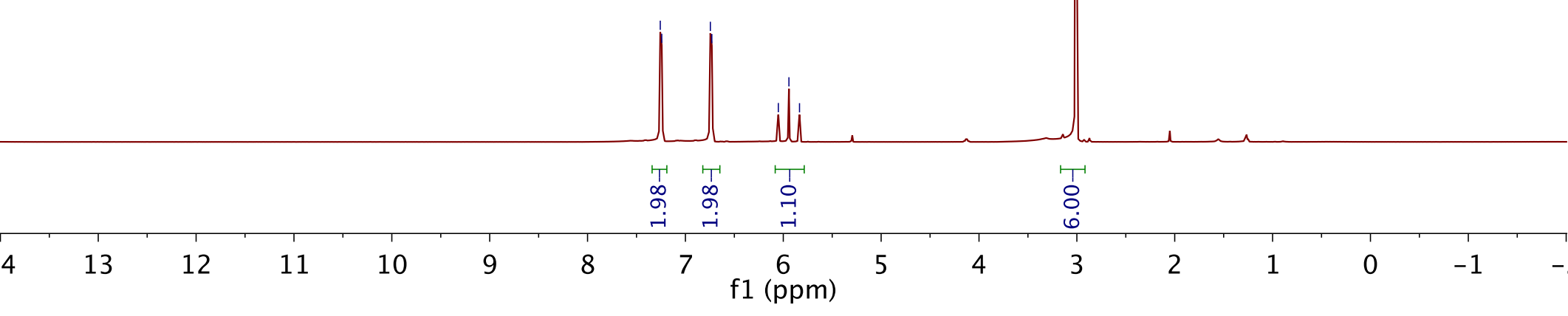


<smiles>CN(C)c1ccc(SC(=O)C(F)F)cc1</smiles>

$1 e$

${ }^{13} \mathrm{C}$ NMR

mo요

नंनंब

걱

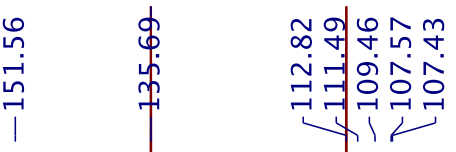

우요

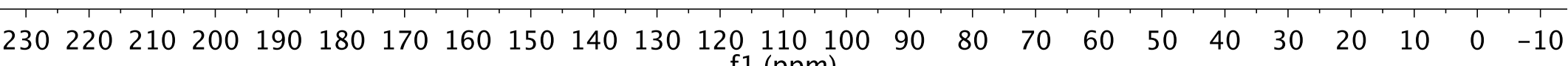

f1 (ppm) 
<smiles>CN(C)c1ccc(SC(=O)C(F)F)cc1</smiles>

$1 e$

${ }^{19} \mathrm{~F}$ NMR

ริ

$\stackrel{m}{\sim}$

I

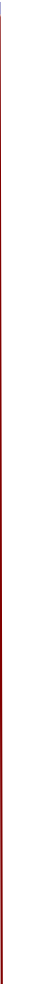

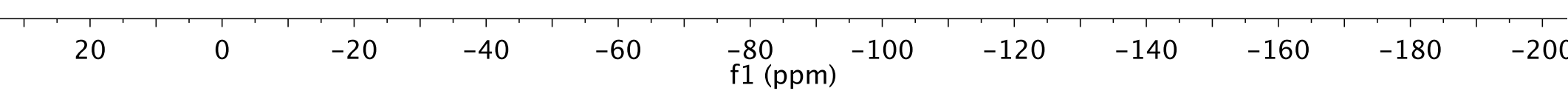


<smiles>O=C(Sc1ccc2ccccc2c1)C(F)F</smiles>

$1 f$

${ }^{1} \mathrm{H}$ NMR
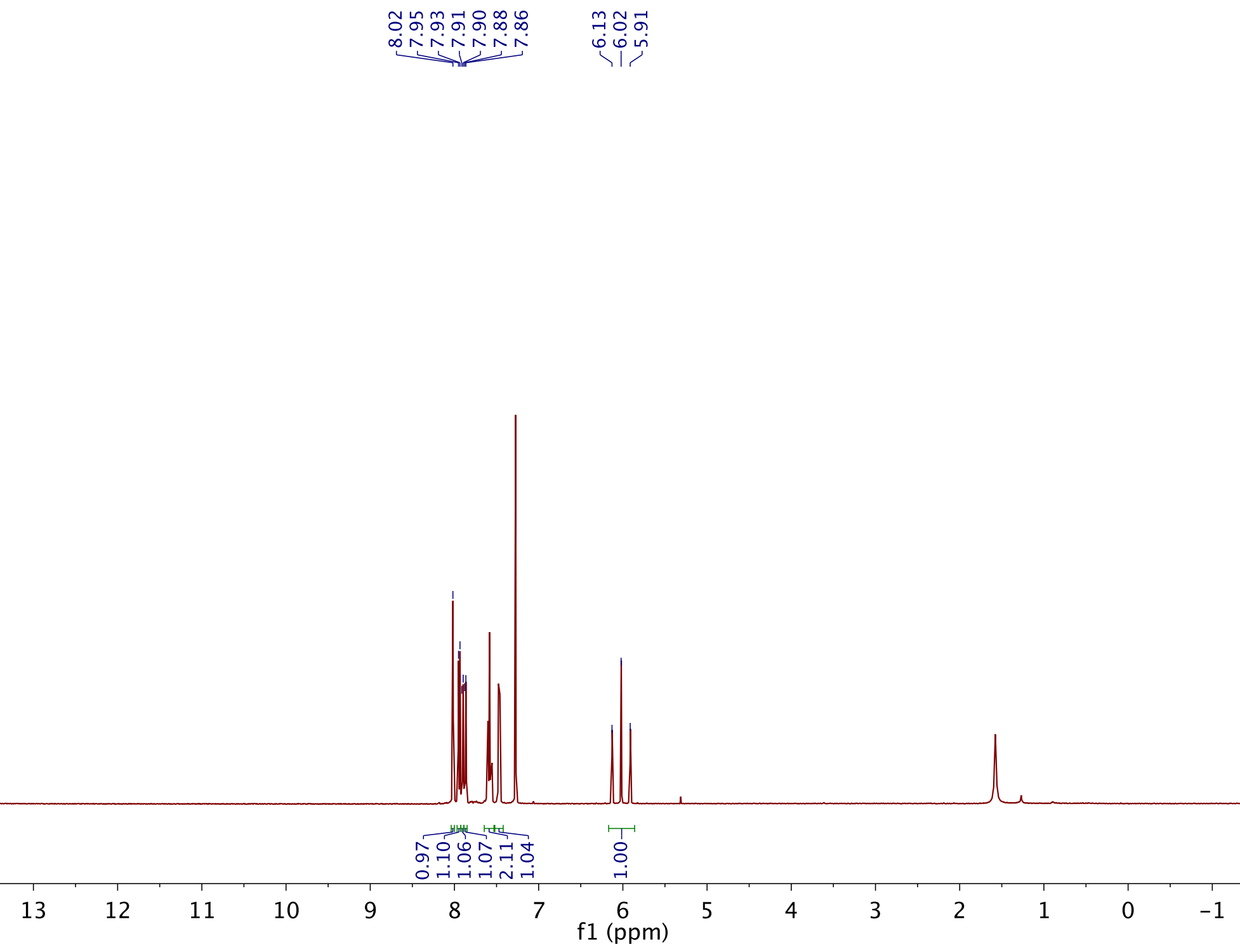
<smiles>O=C(Sc1ccc2ccccc2c1)C(F)F</smiles>

$1 f$

${ }^{19} \mathrm{~F}$ NMR

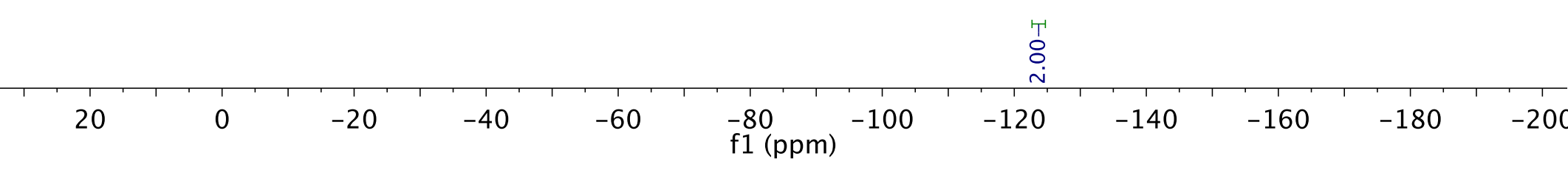


<smiles>O=C(Sc1ccc(F)cc1)C(F)F</smiles>

$1 \mathrm{~g}$

${ }^{1} \mathrm{H}$ NMR

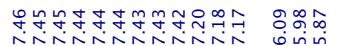

11

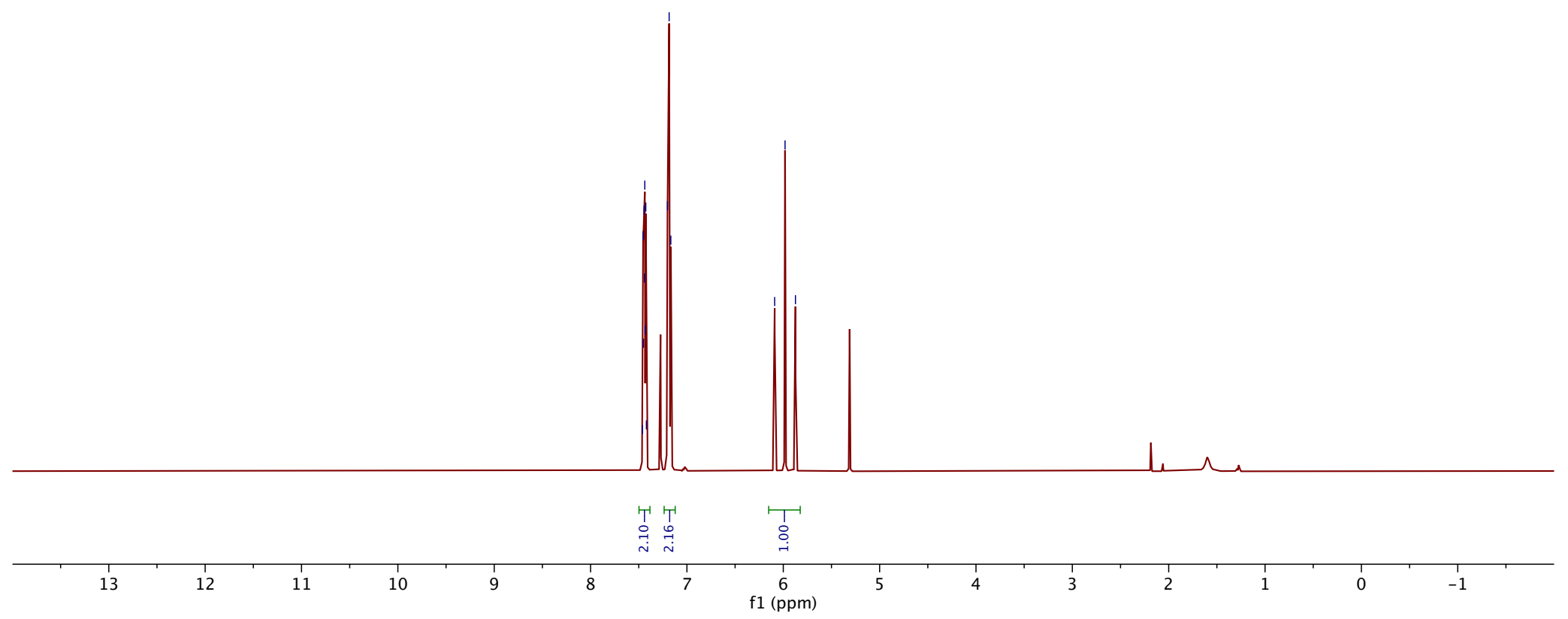


<smiles>O=C(Sc1ccc(F)cc1)C(F)F</smiles>

$1 \mathrm{~g}$

${ }^{13} \mathrm{C}$ NMR

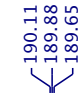

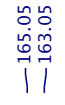

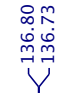

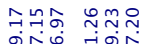

अंज
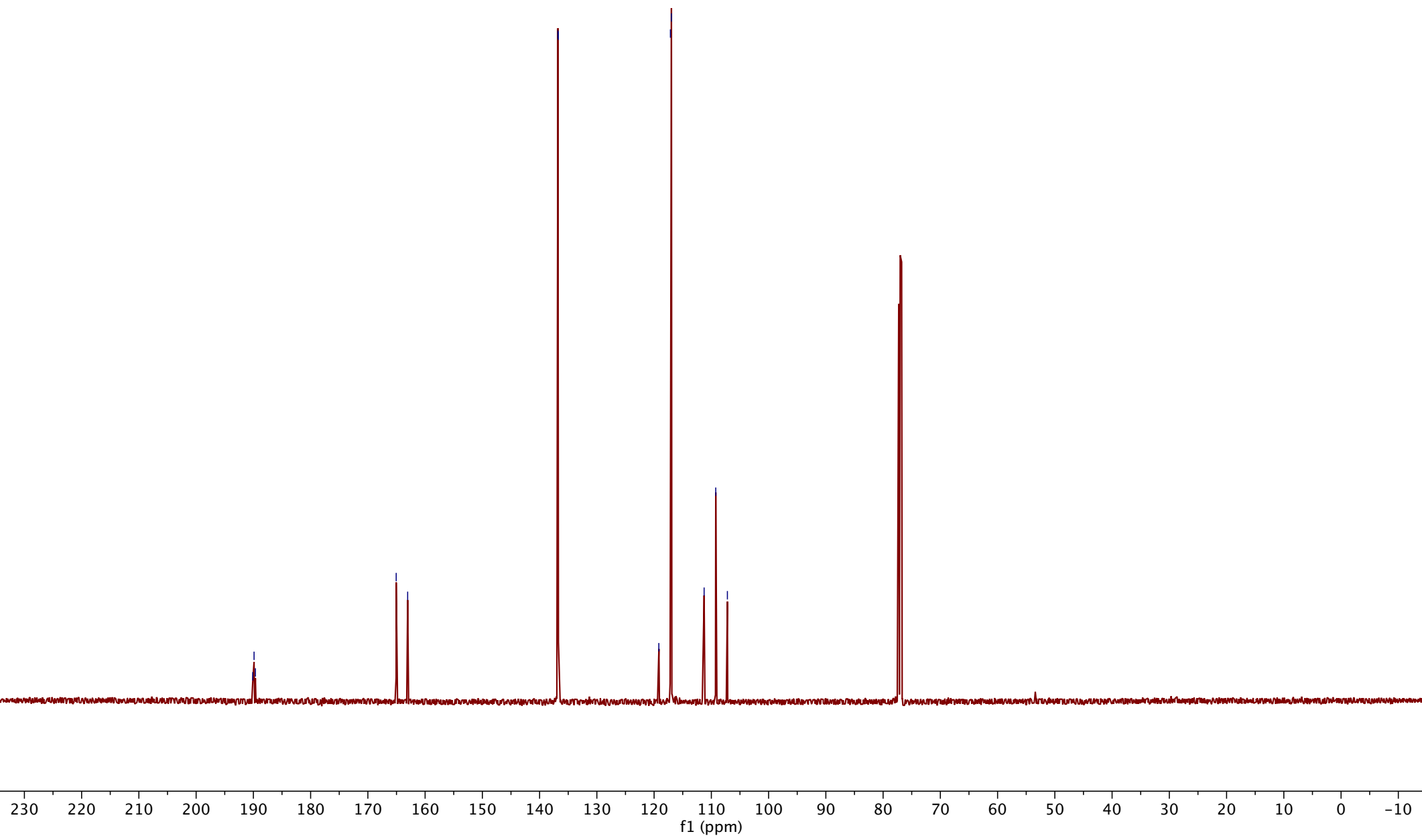
<smiles>O=C(Sc1ccc(F)cc1)C(F)F</smiles>

$1 \mathrm{~g}$

${ }^{19} \mathrm{~F}$ NMR

\begin{tabular}{|c|c|c|c|c|c|c|c|c|c|c|c|c|c|c|c|c|c|c|c|c|c|}
\hline & & & & & & & & & & & & & $\begin{array}{l}y \\
y \\
o \\
o\end{array}$ & 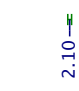 & & & & & & & \\
\hline 20 & 10 & 0 & -10 & -20 & -30 & -40 & -50 & -60 & -70 & $\begin{array}{r}-80 \\
\text { f1 }\end{array}$ & $\begin{array}{l}-90 \\
\mathrm{~m})\end{array}$ & -100 & -110 & -120 & -130 & -140 & -150 & -160 & -170 & -180 & -190 \\
\hline
\end{tabular}


<smiles>COC(=O)c1ccc(SC(=O)C(F)F)cc1</smiles>

$1 \mathrm{~h}$

${ }^{1} \mathrm{H}$ NMR

Y

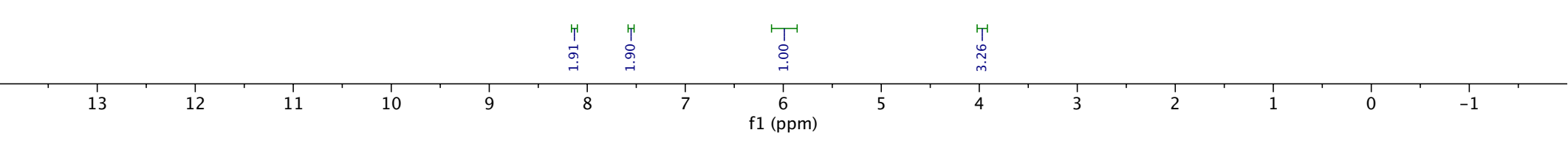


<smiles>COC(=O)c1ccc(SC(=O)C(F)F)cc1</smiles>

$1 \mathrm{~h}$

${ }^{13} \mathrm{C}$ NMR

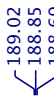

7
-7
0
0
$\mid 7$

유ำ

의

우웅

(1)

기

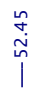

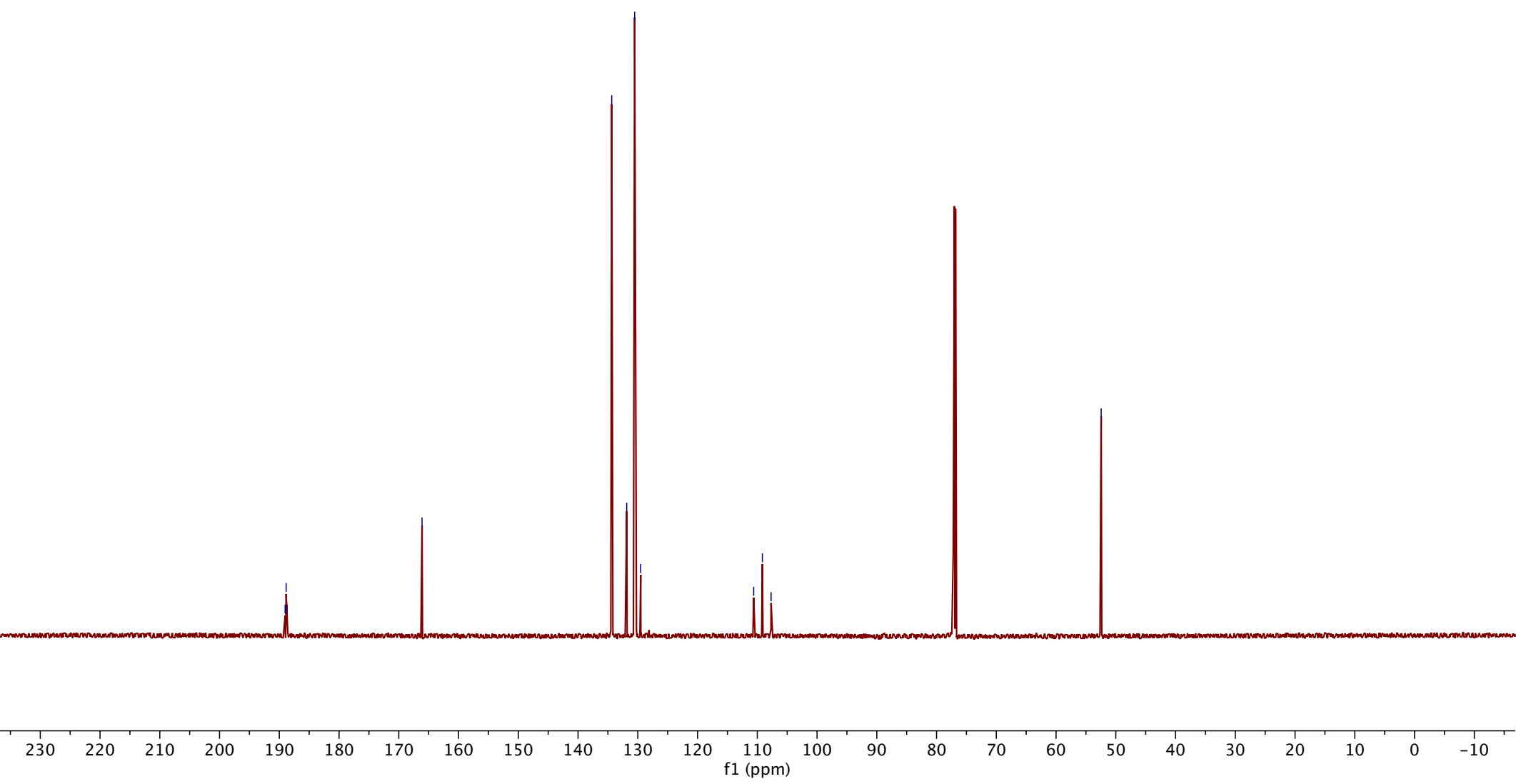


<smiles>CN(C(=O)C(F)F)c1ccc(SC(=O)C(F)F)cc1</smiles>

$1 \mathrm{i}$

${ }^{1} \mathrm{H}$ NMR

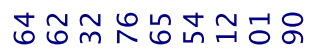

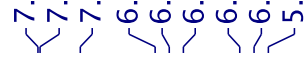

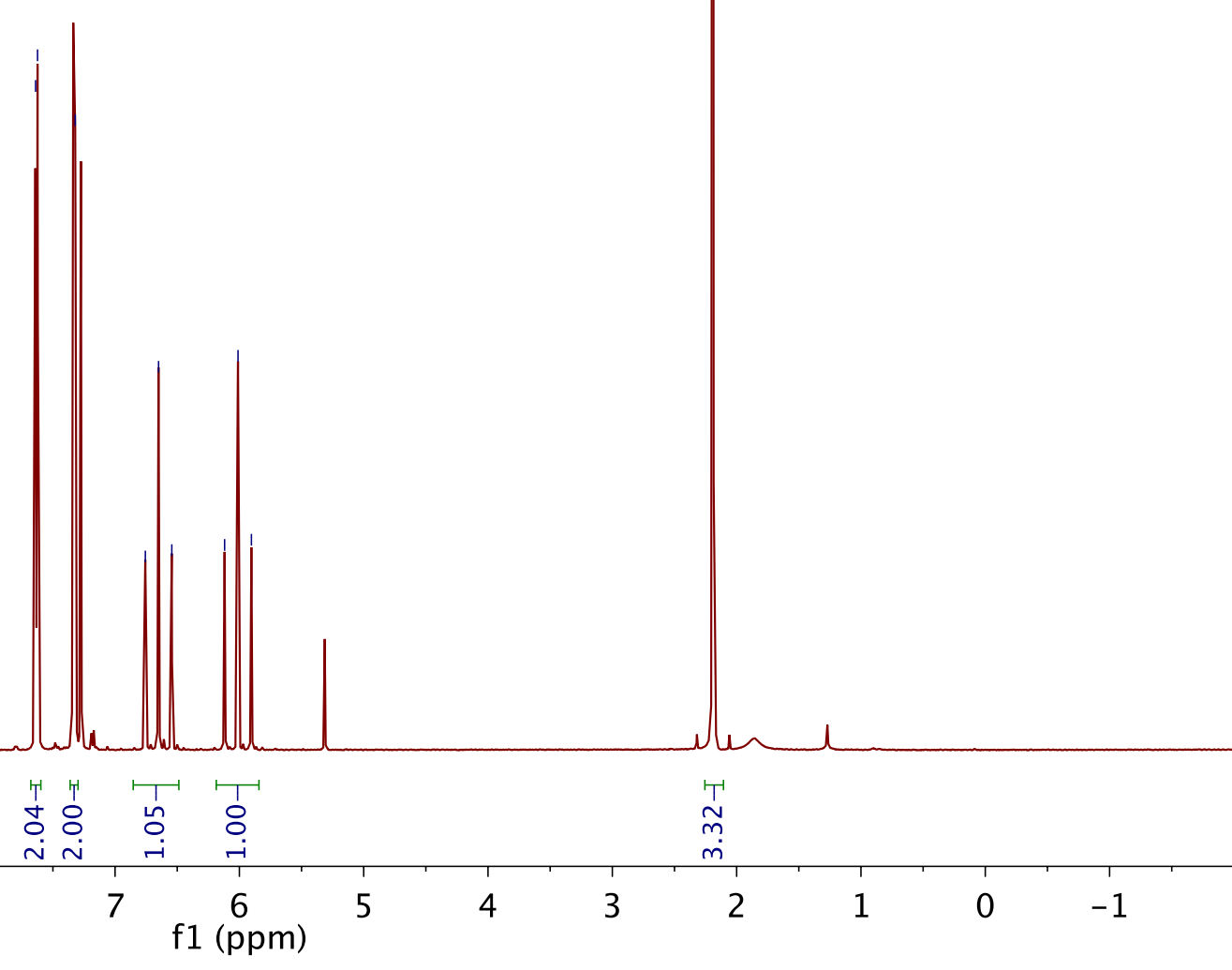


<smiles>CN(C(=O)C(F)F)c1ccc(SC(=O)C(F)F)cc1</smiles>

$1 \mathrm{i}$

${ }^{13} \mathrm{C}$ NMR

รำำ ลำํํำ

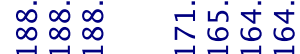

.

구교

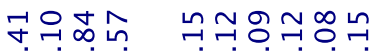

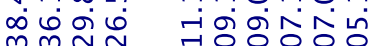

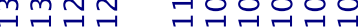

१ग्रा

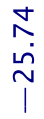

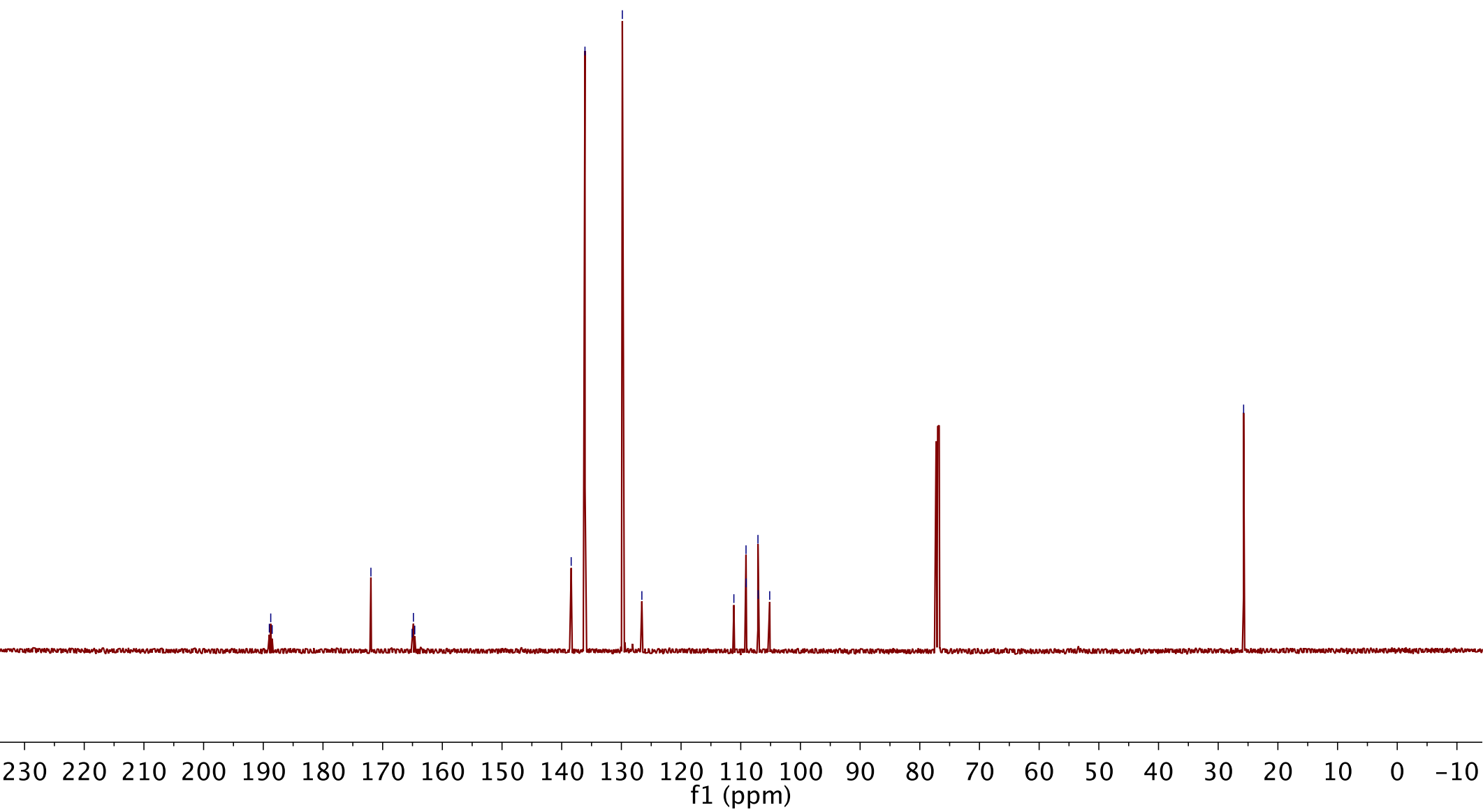


<smiles>CN(C(=O)C(F)F)c1ccc(SC(=O)C(F)F)cc1</smiles>

$1 \mathrm{i}$

${ }^{19} \mathrm{~F}$ NMR

웅ํํำ

mंn்

11

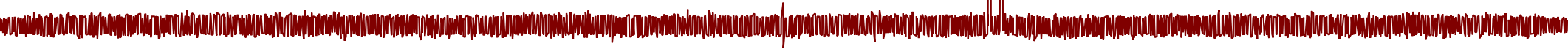

\begin{tabular}{|c|c|c|c|c|c|c|c|c|c|c|c|c|}
\hline & & & & & & & & 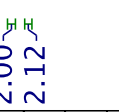 & & & & \\
\hline 30 & 10 & -10 & -30 & -50 & -70 & $\mathrm{f} 1$ (ppm) & -110 & -130 & -150 & -170 & -190 & -210 \\
\hline
\end{tabular}


<smiles>O=C(Sc1ccccc1F)C(F)F</smiles>

${ }^{1} \mathrm{H}$ NMR

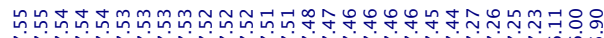

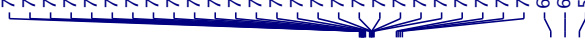

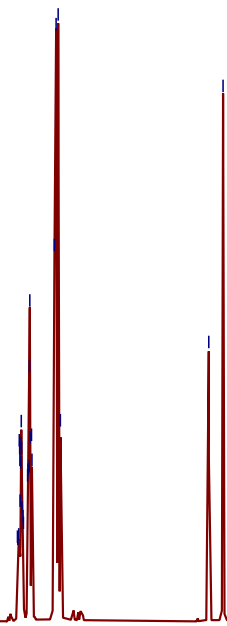

$\mathrm{H}$

\begin{tabular}{|c|c|c|c|c|c|c|c|c|c|c|c|c|c|c|}
\hline & & & & & & & 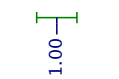 & & & & & & & \\
\hline 13 & 12 & 11 & $\begin{array}{l}1 \\
10\end{array}$ & $\begin{array}{l}1 \\
9\end{array}$ & $\begin{array}{l}1 \\
8\end{array}$ & 7 & $\begin{array}{c}1 \\
6 \\
\mathrm{f} 1(\mathrm{ppm})\end{array}$ & $\begin{array}{l}1 \\
5\end{array}$ & 4 & 3 & 2 & $\begin{array}{l}1 \\
1\end{array}$ & 0 & -1 \\
\hline
\end{tabular}


<smiles>O=C(Sc1ccc(C(F)(F)F)cc1)C(F)F</smiles>

$1 \mathrm{k}$

${ }^{13} \mathrm{C}$ NMR

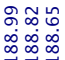

V

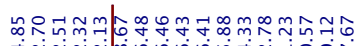

抣

,

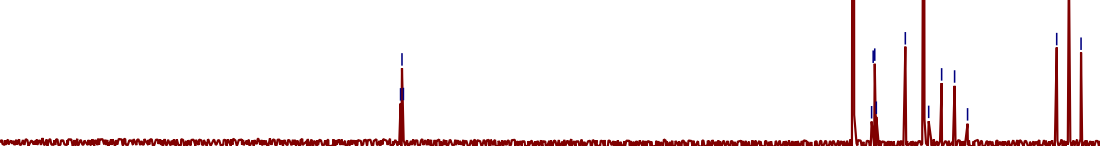

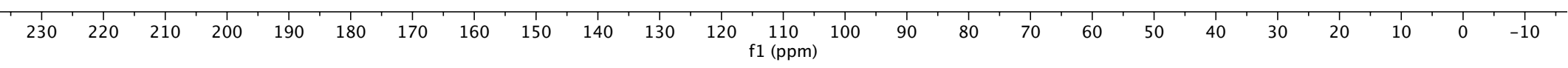


<smiles>O=C(Sc1ccc(C(F)(F)F)cc1)C(F)F</smiles>

$1 \mathrm{k}$

${ }^{19} \mathrm{~F}$ NMR

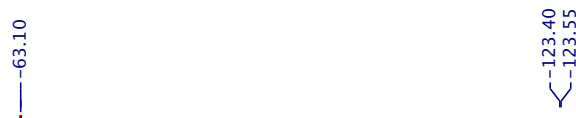

v

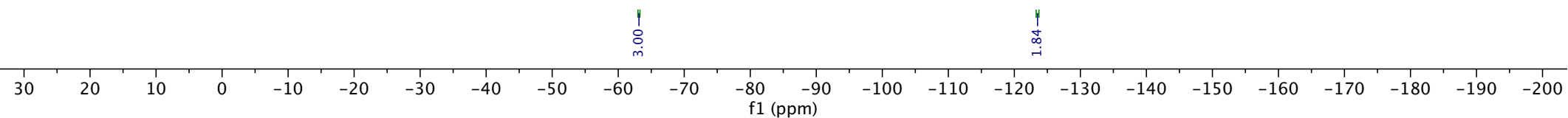


<smiles>O=C(Sc1ccccc1C(F)(F)F)C(F)F</smiles>

11

${ }^{1} \mathrm{H}$ NMR

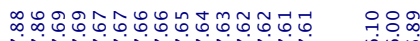

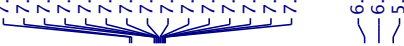

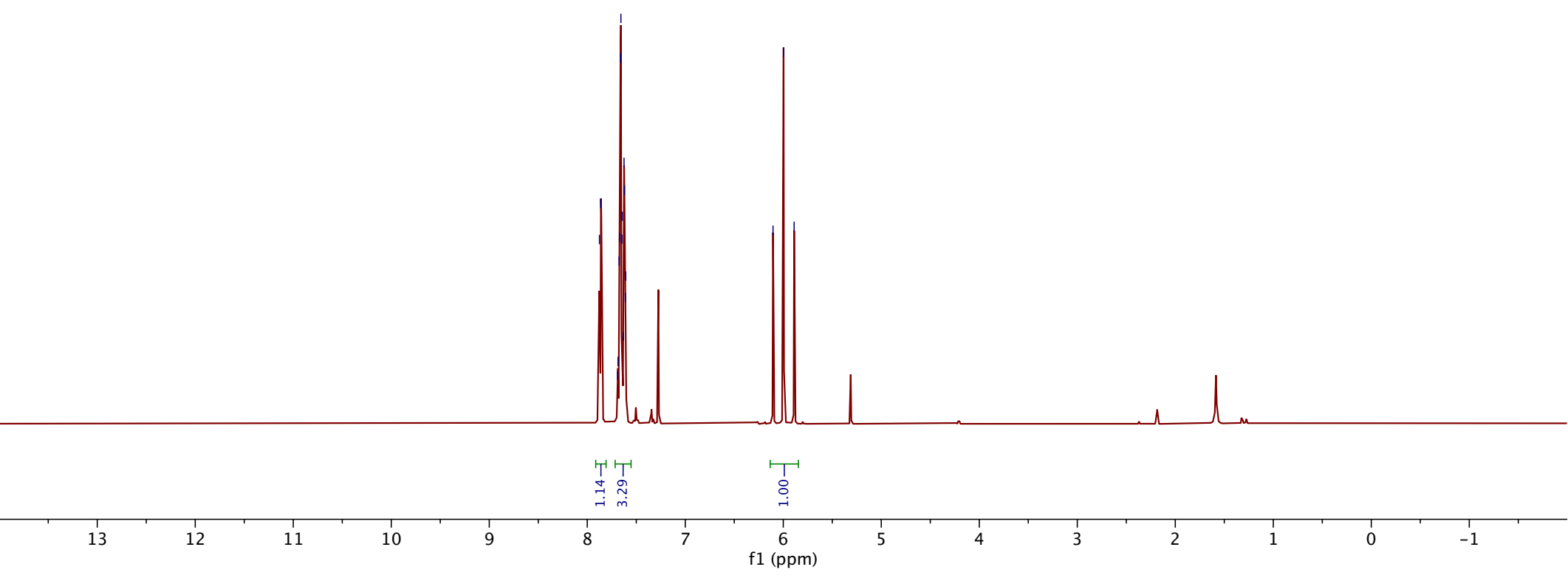


<smiles>O=C(Sc1ccccc1C(F)(F)F)C(F)F</smiles>

11

${ }^{13} \mathrm{C}$ NMR

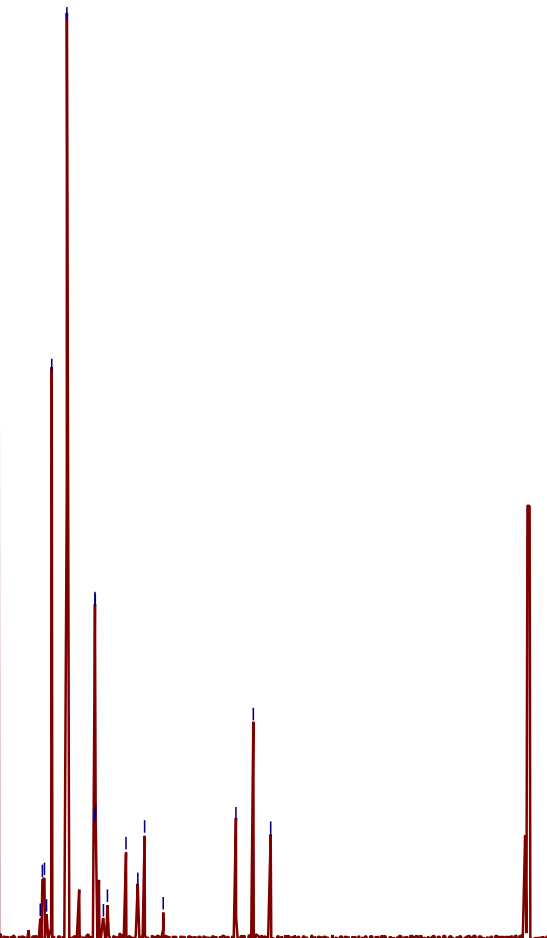

\begin{tabular}{|c|c|c|c|c|c|c|c|c|c|c|c|c|c|c|c|c|c|c|c|c|c|c|c|c|}
\hline 230 & 220 & 210 & 200 & $\begin{array}{c}1 \\
190\end{array}$ & 180 & 170 & 160 & 150 & 140 & 130 & 120 & 110 & 100 & 90 & 80 & 70 & 60 & 50 & 40 & 30 & 20 & 10 & $\begin{array}{l}1 \\
0\end{array}$ & -10 \\
\hline
\end{tabular}


<smiles>Cc1ccccc1SC(=O)C(F)F</smiles>

$1 \mathrm{~m}$

${ }^{1} \mathrm{H}$ NMR

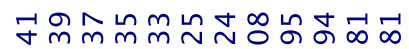

수수숫ำ

皮 
<smiles>Cc1ccccc1SC(=O)C(F)F</smiles>

$1 \mathrm{~m}$

${ }^{13} \mathrm{C}$ NMR
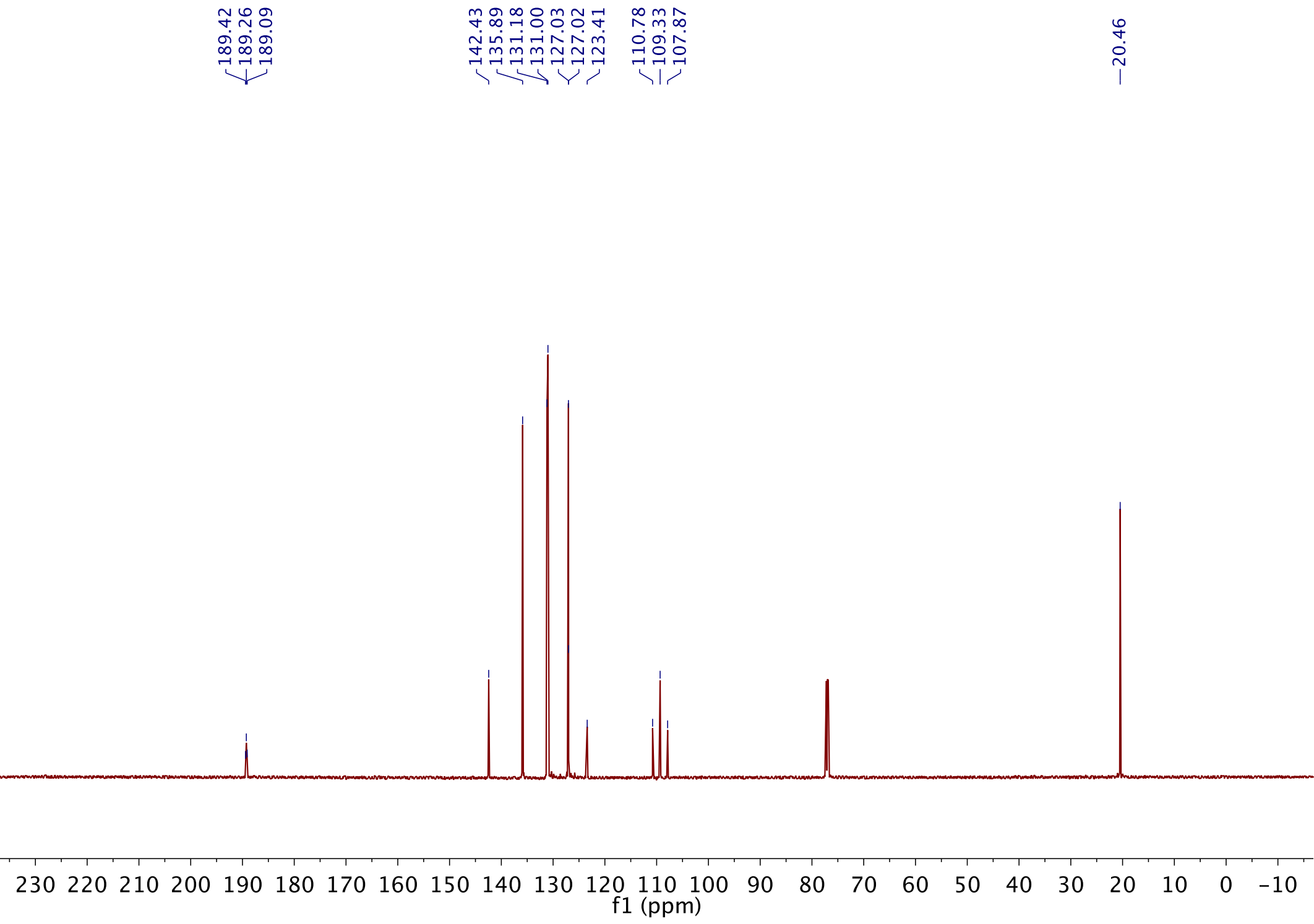
<smiles>Cc1ccccc1SC(=O)C(F)F</smiles>

$1 \mathrm{~m}$

${ }^{19} \mathrm{~F}$ NMR

商

$\ddot{n} \ddot{\sim}$

그

'

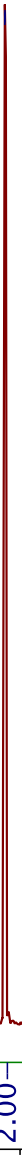

$-20-40 \quad-60$

$-80$

f1 (ppm)

$-100-120$

$-140 \quad-160$

$-180$

$-2$ 


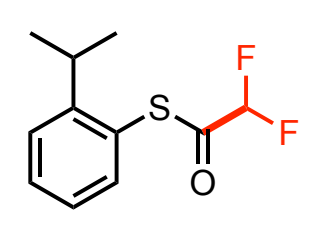

1n

${ }^{13} \mathrm{C}$ NMR
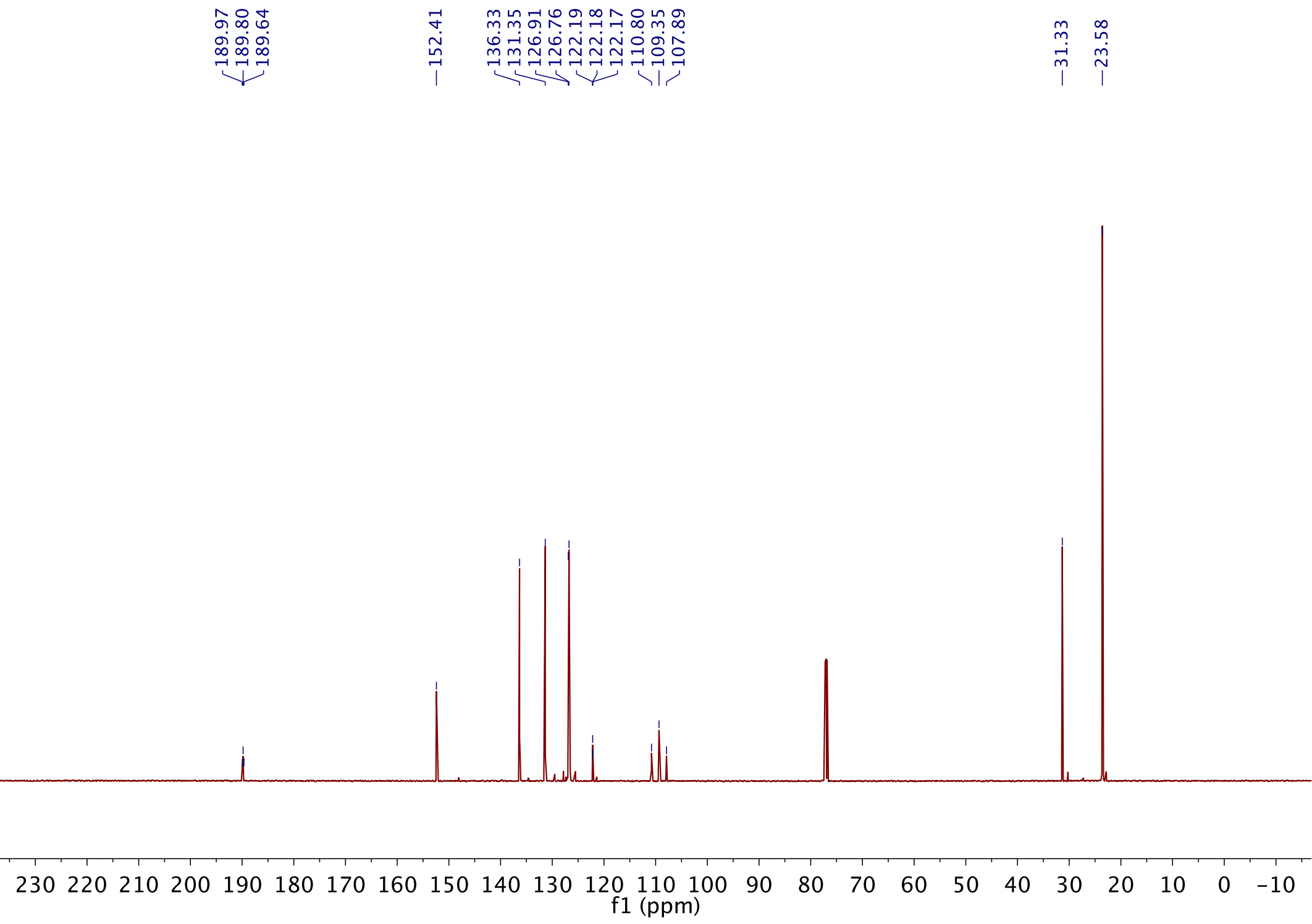


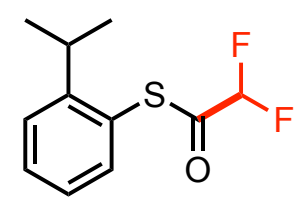

$1 \mathrm{n}$

${ }^{19} \mathrm{~F}$ NMR
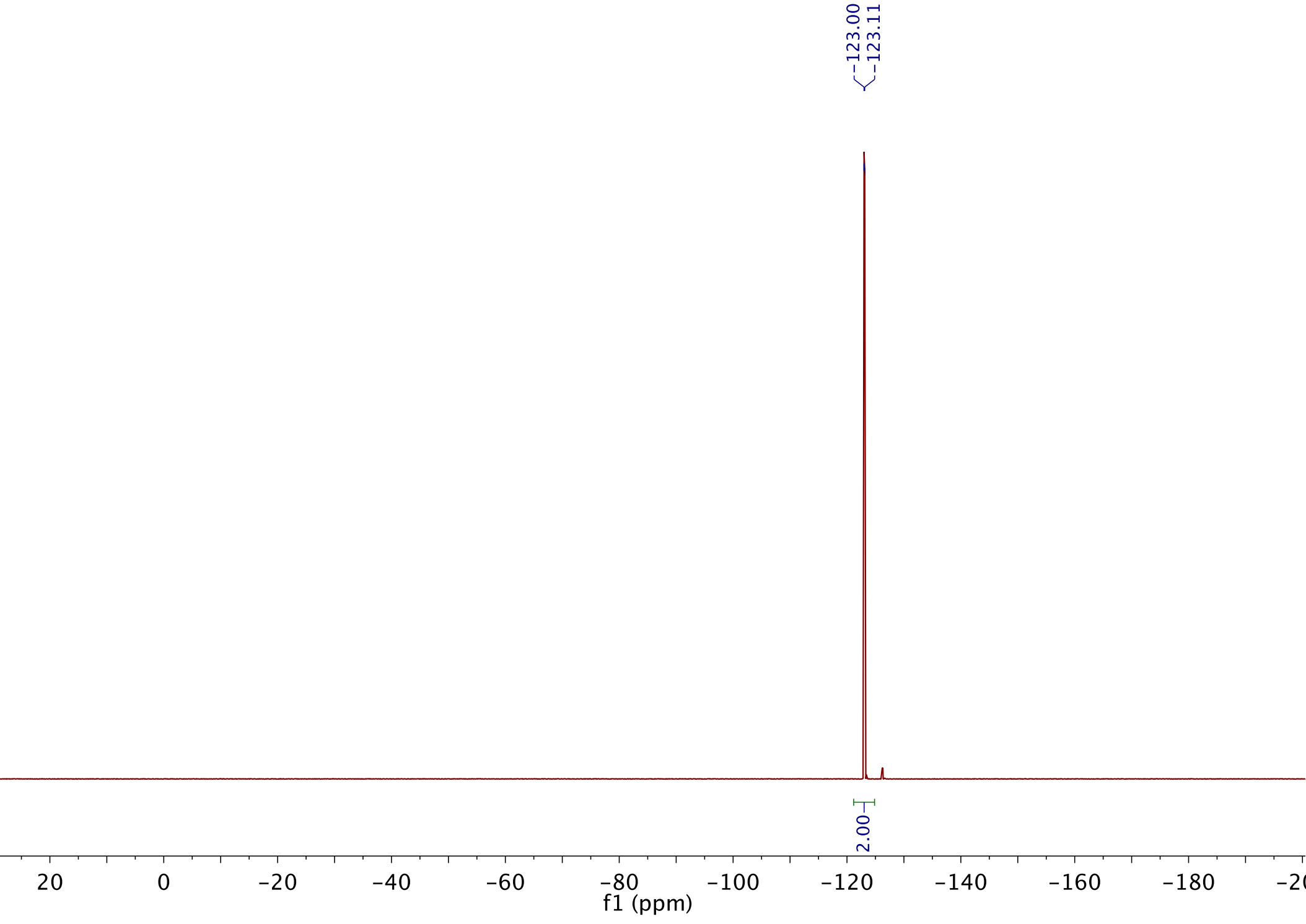


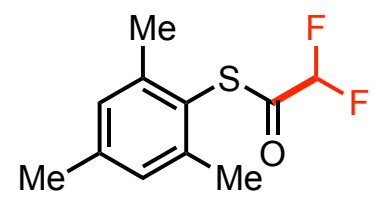

10

${ }^{1} \mathrm{H}$ NMR
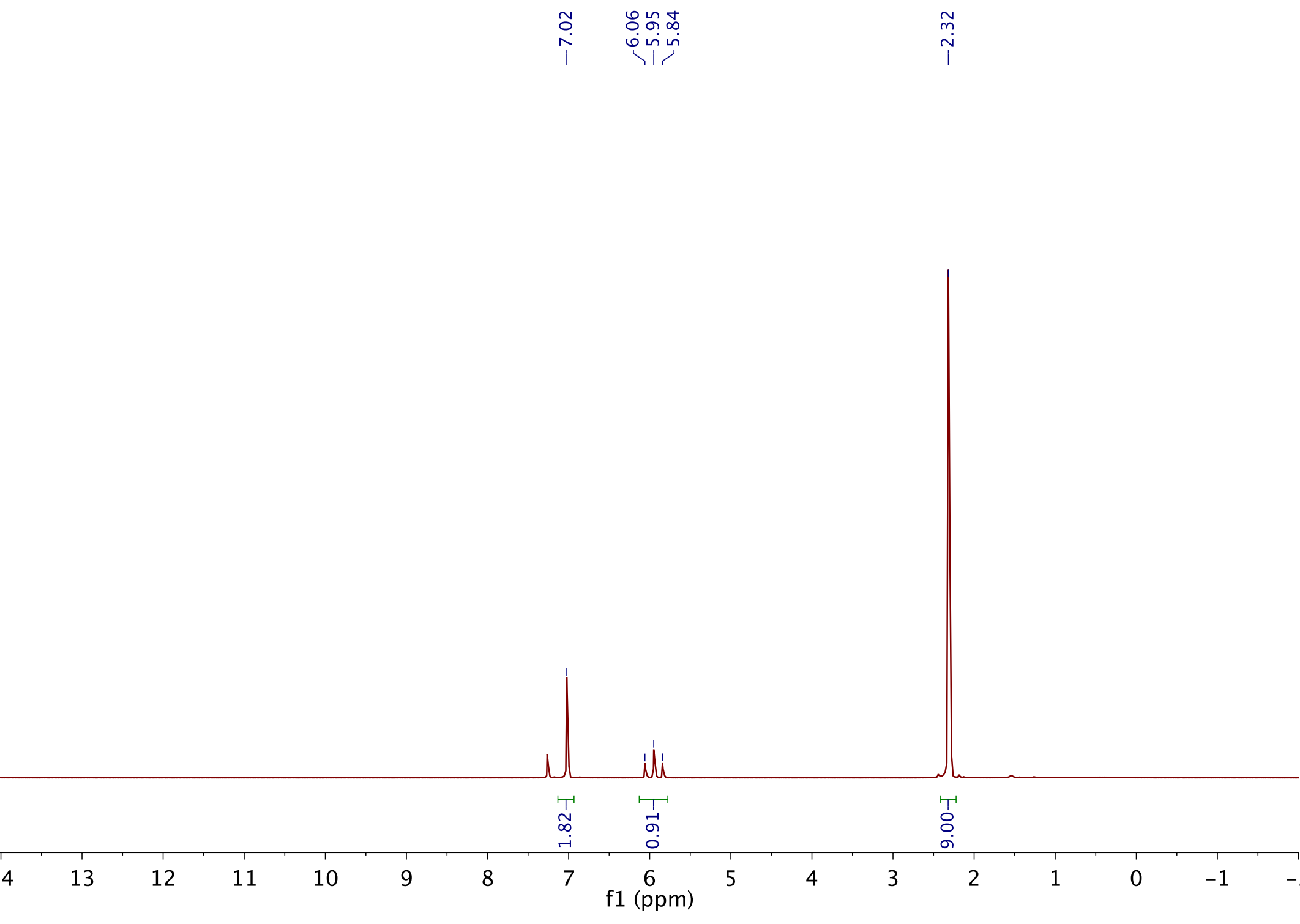
<smiles>Cc1cc(C)c(SC(=O)C(F)F)c(C)c1</smiles>

10

${ }^{13} \mathrm{C}$ NMR

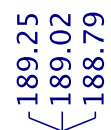

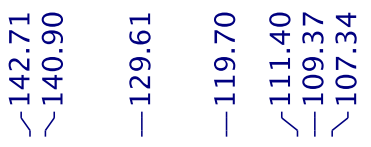

$\underset{\sim}{\stackrel{2}{\sim}}$

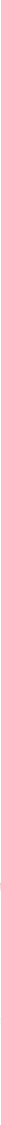




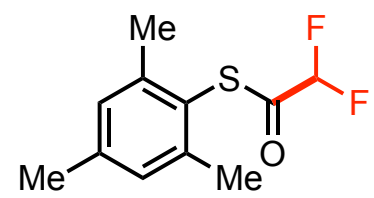

10

${ }^{19} \mathrm{~F}$ NMR

๓

กิ

$\sqrt{1}$ 
<smiles>O=C(SCCc1ccccc1)C(F)F</smiles>

$1 p$

${ }^{1} \mathrm{H}$ NMR

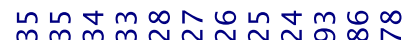

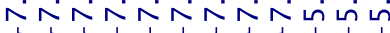

กิํํำดัดั

mंxiñ

?

ए

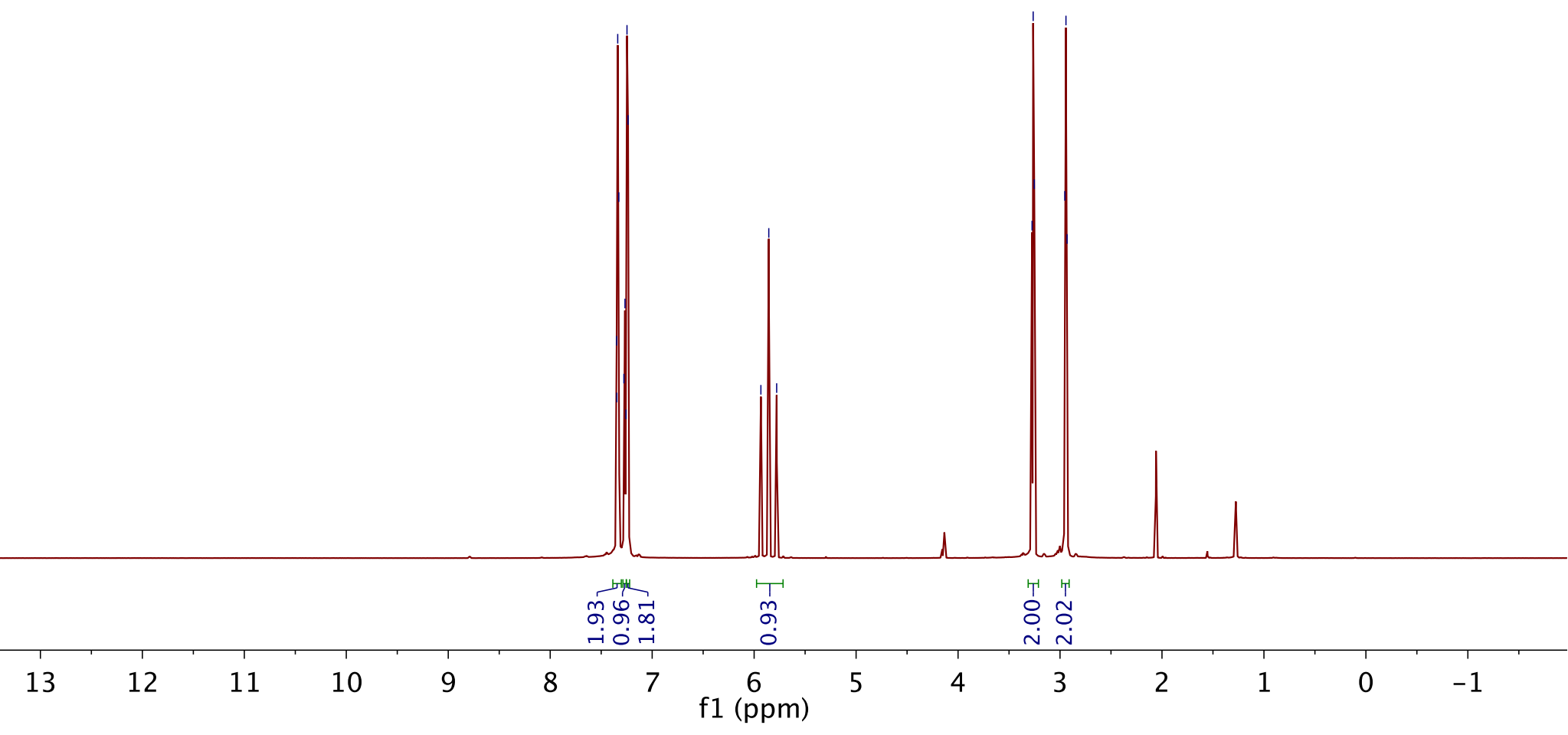


<smiles>O=C(SCCc1ccccc1)C(F)F</smiles>

$1 p$

${ }^{13} \mathrm{C}$ NMR

نَ

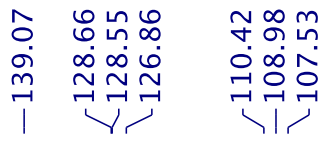

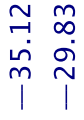

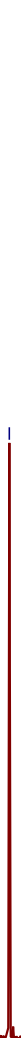
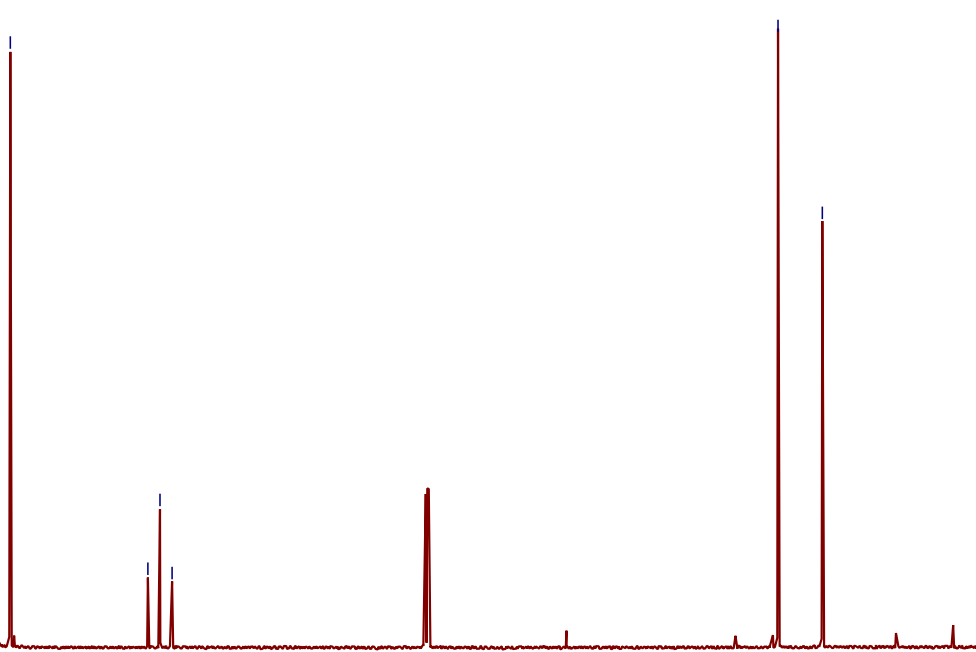

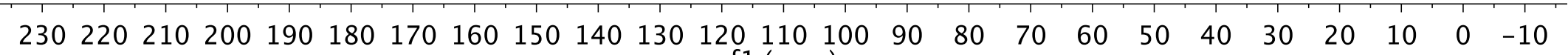
f1 (ppm) 
<smiles>O=C(SCCc1ccccc1)C(F)F</smiles>

$1 p$

${ }^{19} \mathrm{~F}$ NMR

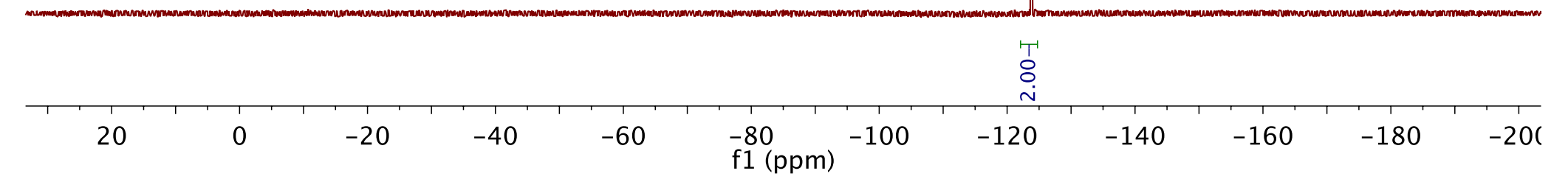


<smiles>O=C(SCc1ccccc1)C(F)F</smiles>

$1 \mathrm{q}$

${ }^{1} \mathrm{H}$ NMR

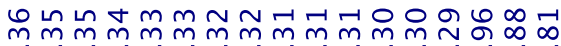

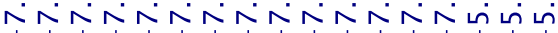

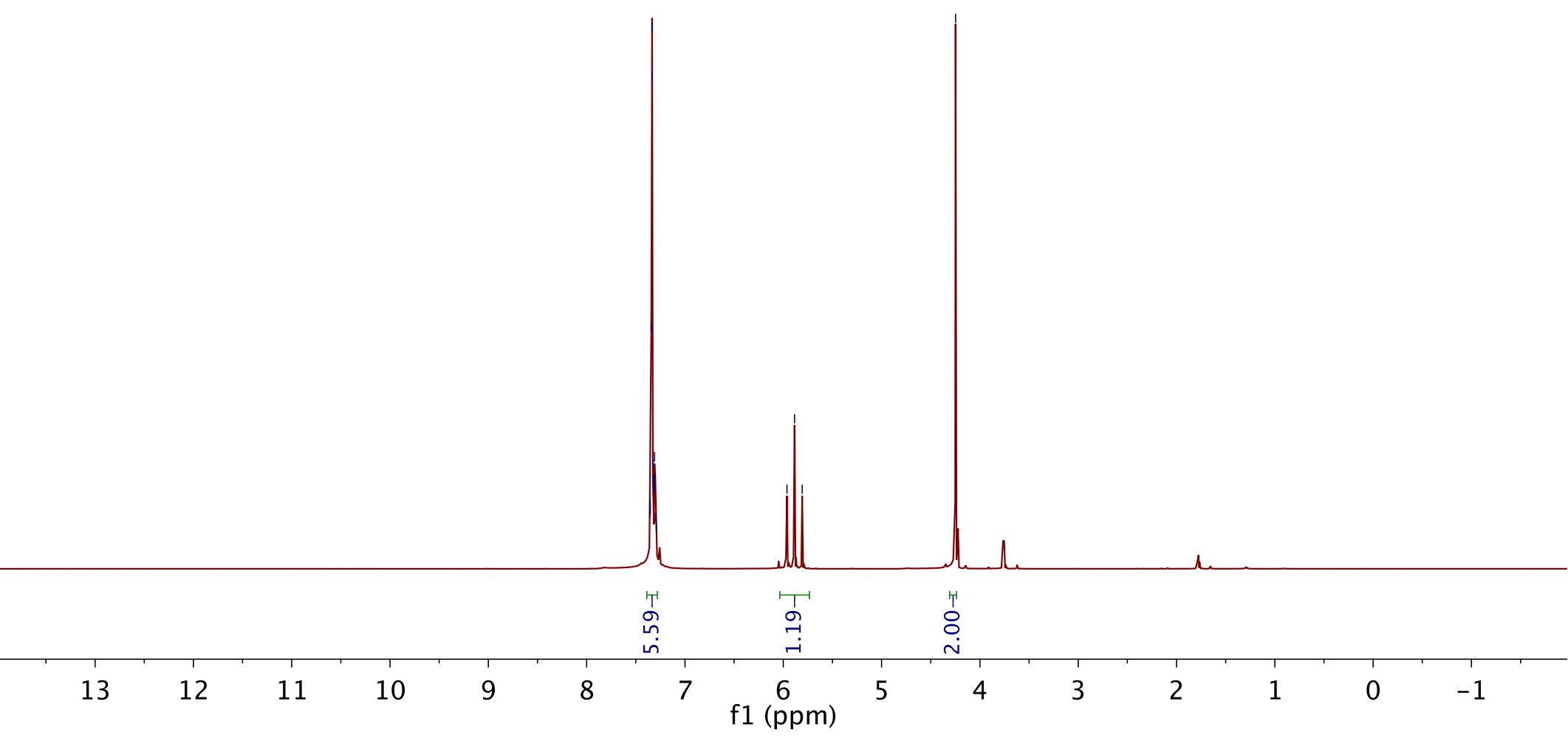


<smiles>O=C(SCc1ccccc1)C(F)F</smiles>

$1 q$

${ }^{13} \mathrm{C}$ NMR
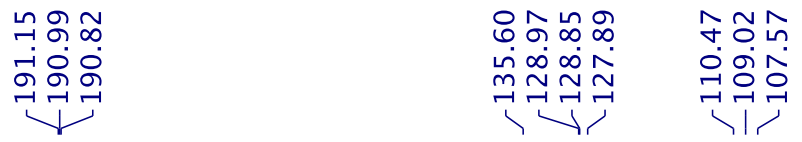

$\stackrel{\hat{N}}{n}$

\section{(}

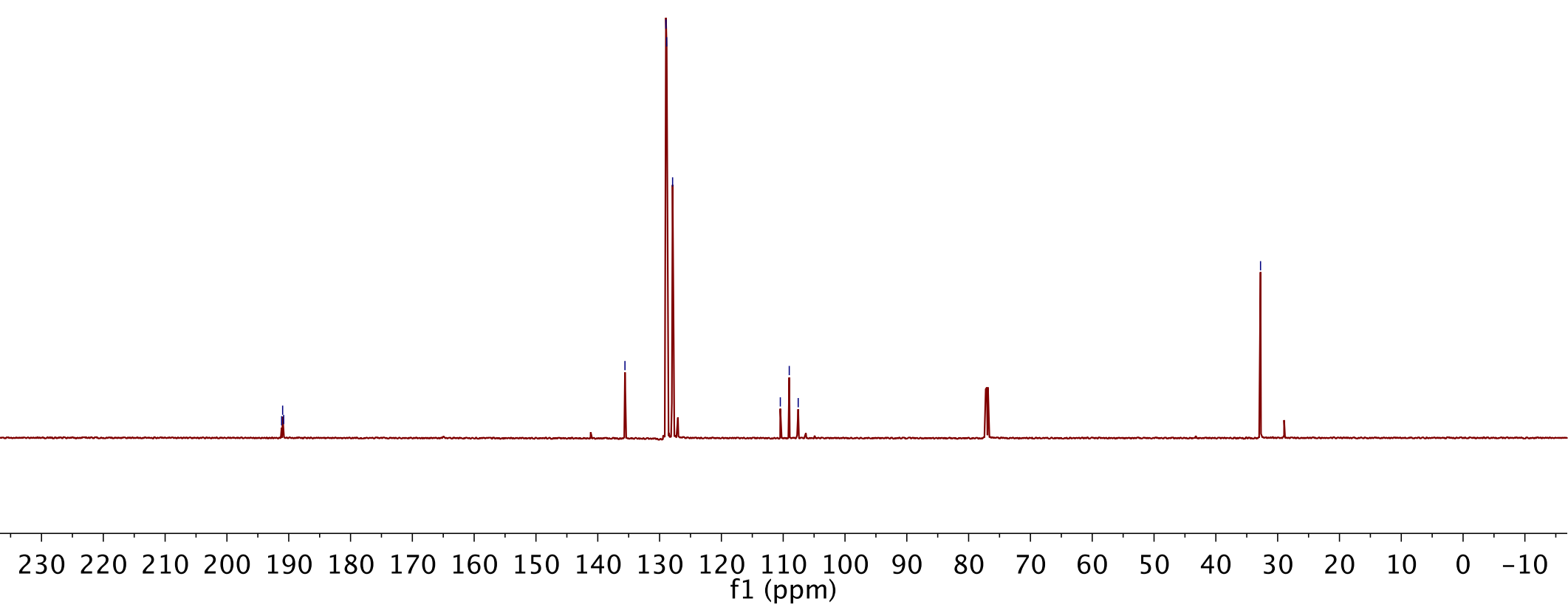


<smiles>O=C(SCc1ccccc1)C(F)F</smiles>

$1 q$

${ }^{19} \mathrm{~F}$ NMR

$N \infty$

$\stackrel{m}{\sim}$

i

. 


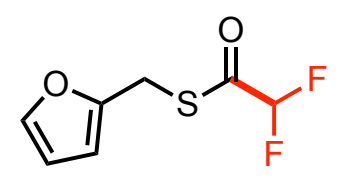

$1 r$

${ }^{1} \mathrm{H}$ NMR

mํำm

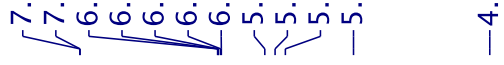

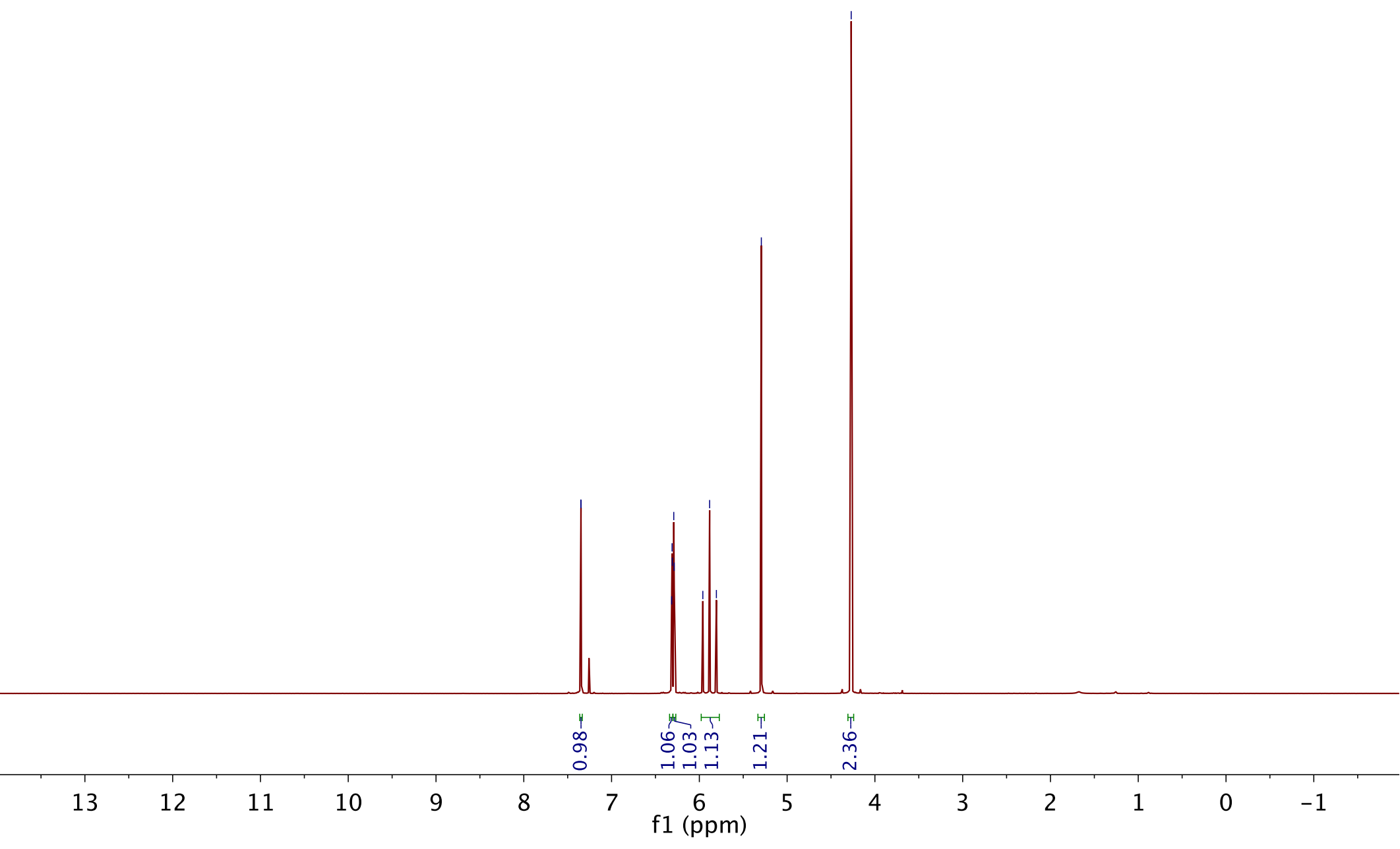


<smiles>O=C(SCc1ccco1)C(F)F</smiles>

$1 \mathrm{r}$

${ }^{13} \mathrm{C}$ NMR

ํำกำ

ดำ ดำ

计

$\mathscr{+}$

ㅊํํㅇํㅇำ

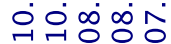

$\neg-\pi-r^{-1}$

$\stackrel{1}{\rightarrow} \underset{+}{\stackrel{1}{+}}$

ry

in

$\stackrel{+}{\sim}$

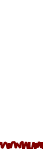

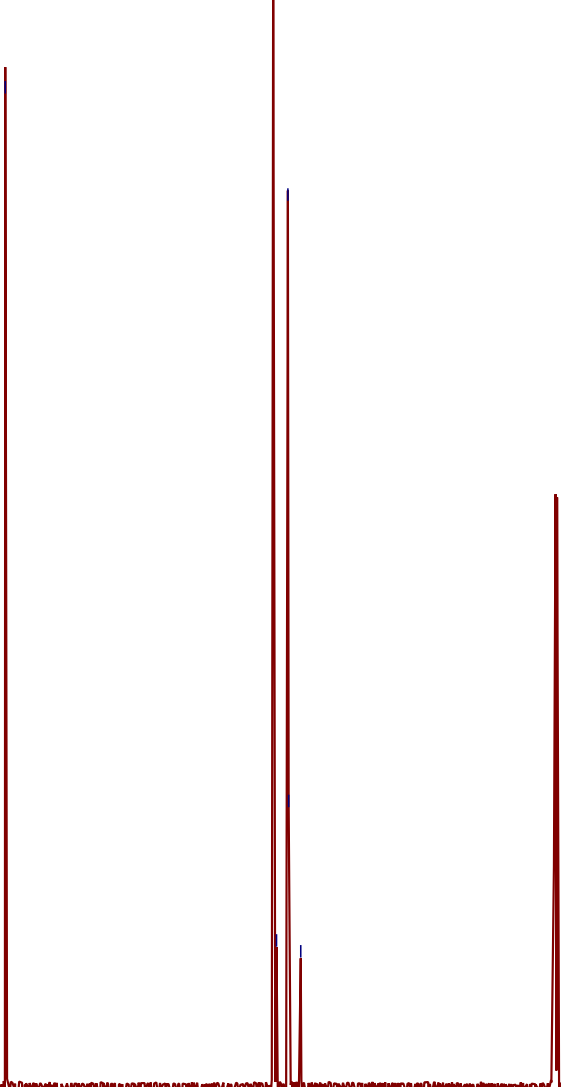

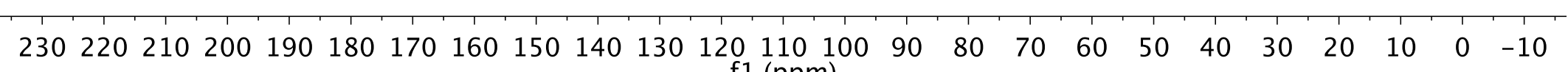

f1 (ppm) 
<smiles>O=C(SCc1ccco1)C(F)F</smiles>

$1 r$

${ }^{19} \mathrm{~F}$ NMR
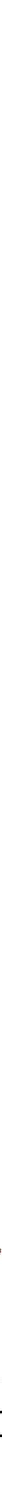
<smiles>O=C(SC1CCCCC1)C(F)F</smiles>

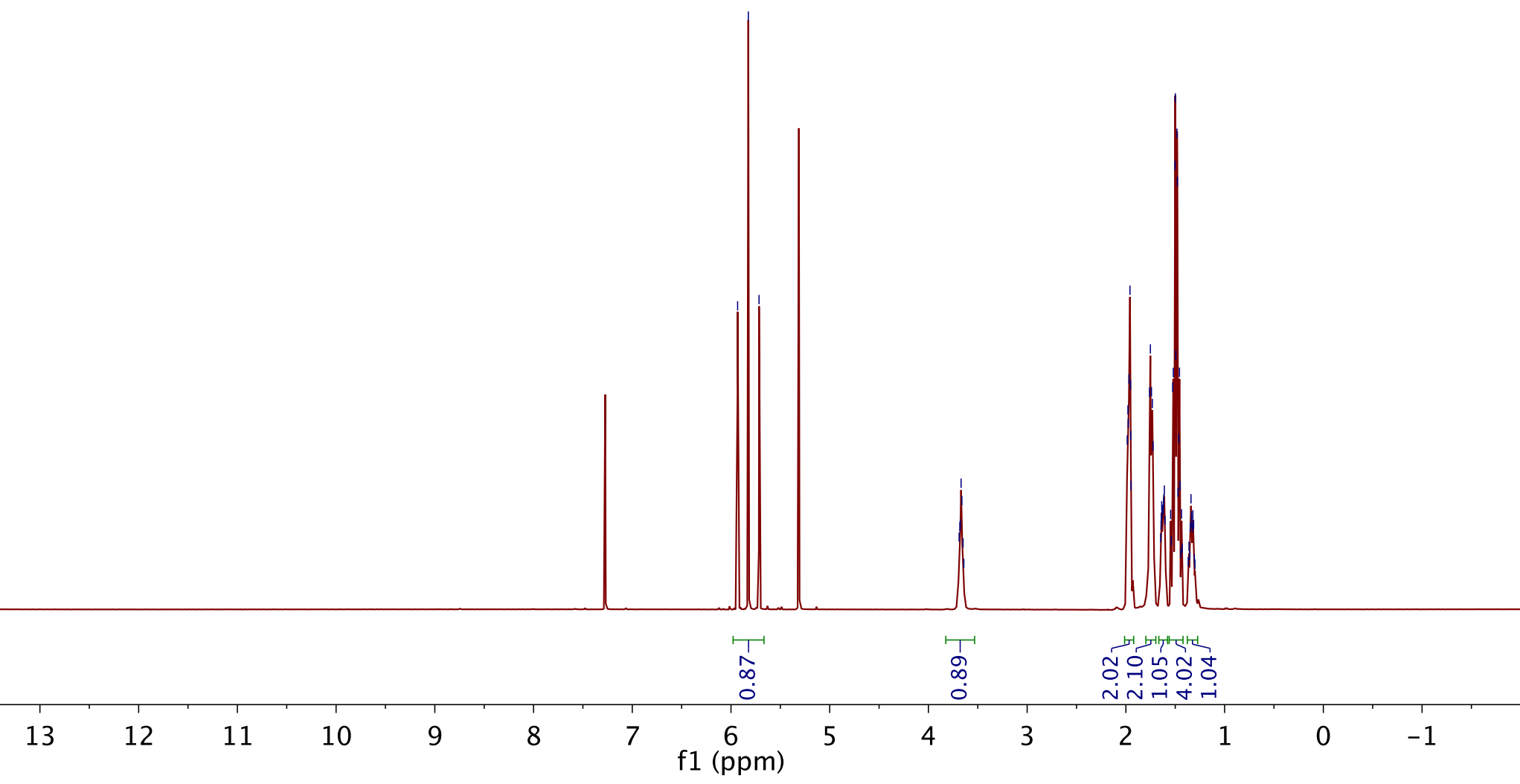


<smiles>O=C(SC1CCCCC1)C(F)F</smiles>

$1 \mathrm{~s}$

${ }^{13} \mathrm{C}$ NMR

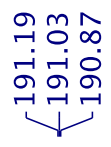

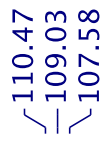

도 스욨

チ $\quad$ กำํำ

$i \sim$

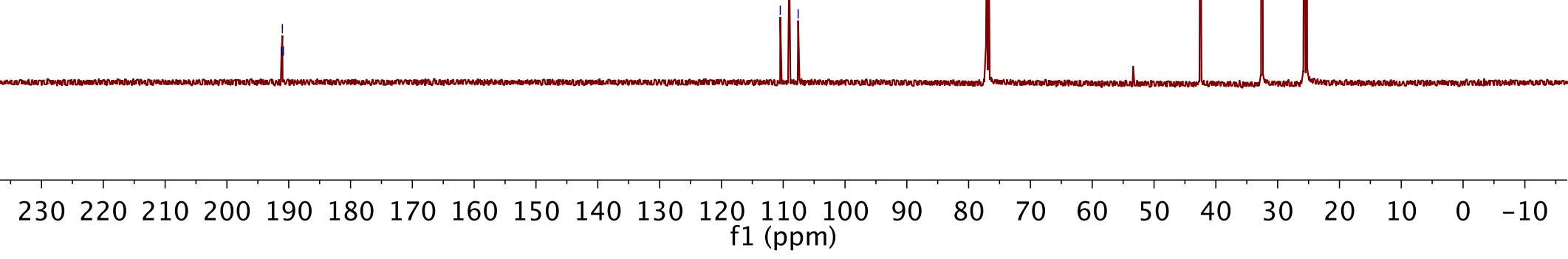


<smiles>O=C(SC1CCCCC1)C(F)F</smiles>

$1 \mathrm{~s}$

${ }^{19} \mathrm{~F}$ NMR

कूล

$m \sim$

$\underset{-7}{\sim}$

I

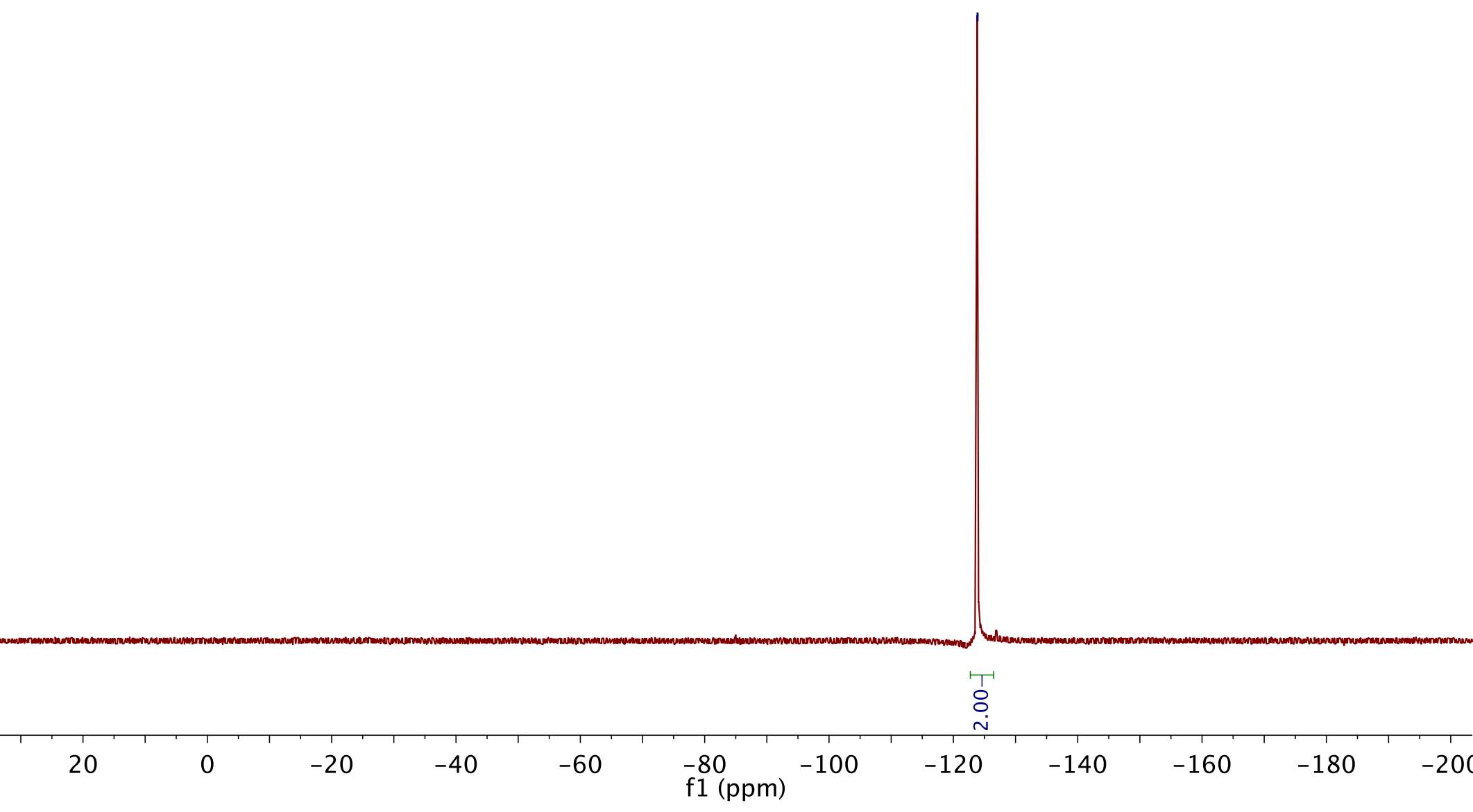


<smiles>O=C(SC12CC3CC(CC(C3)C1)C2)C(F)F</smiles>

$1 \mathrm{t}$

${ }^{1} \mathrm{H}$ NMR

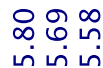

ini in

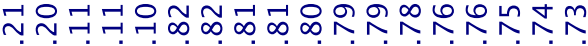

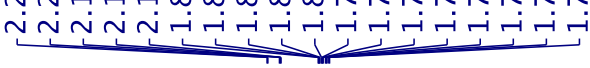

\section{-}

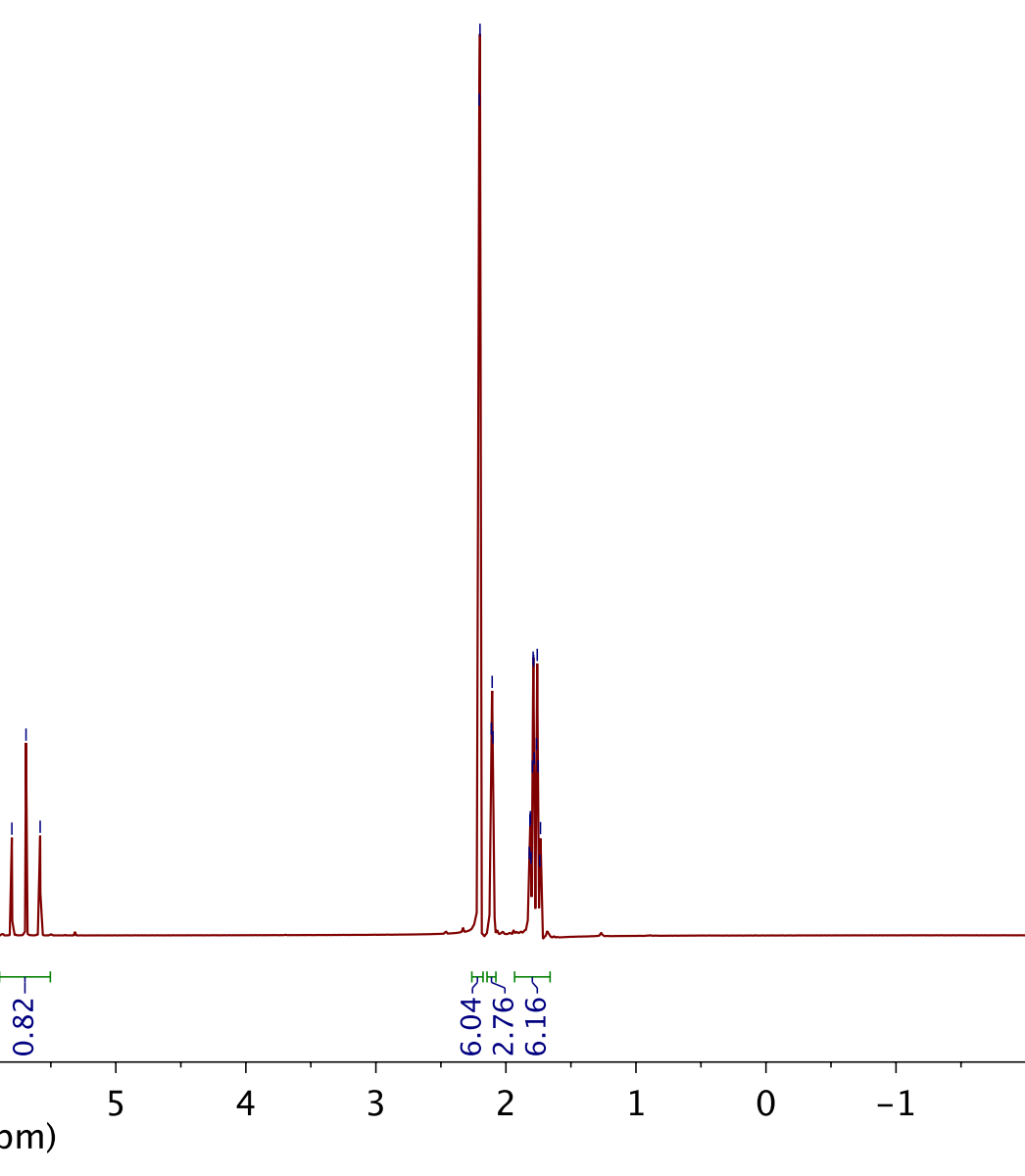




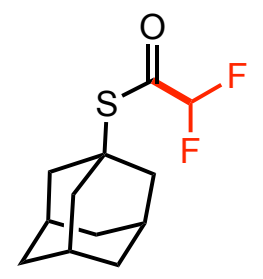

$1 \mathrm{t}$

${ }^{13} \mathrm{C}$ NMR

ิํํ용

ட용

r

$m \infty m$

욕우

기

옹․․

चं

अ
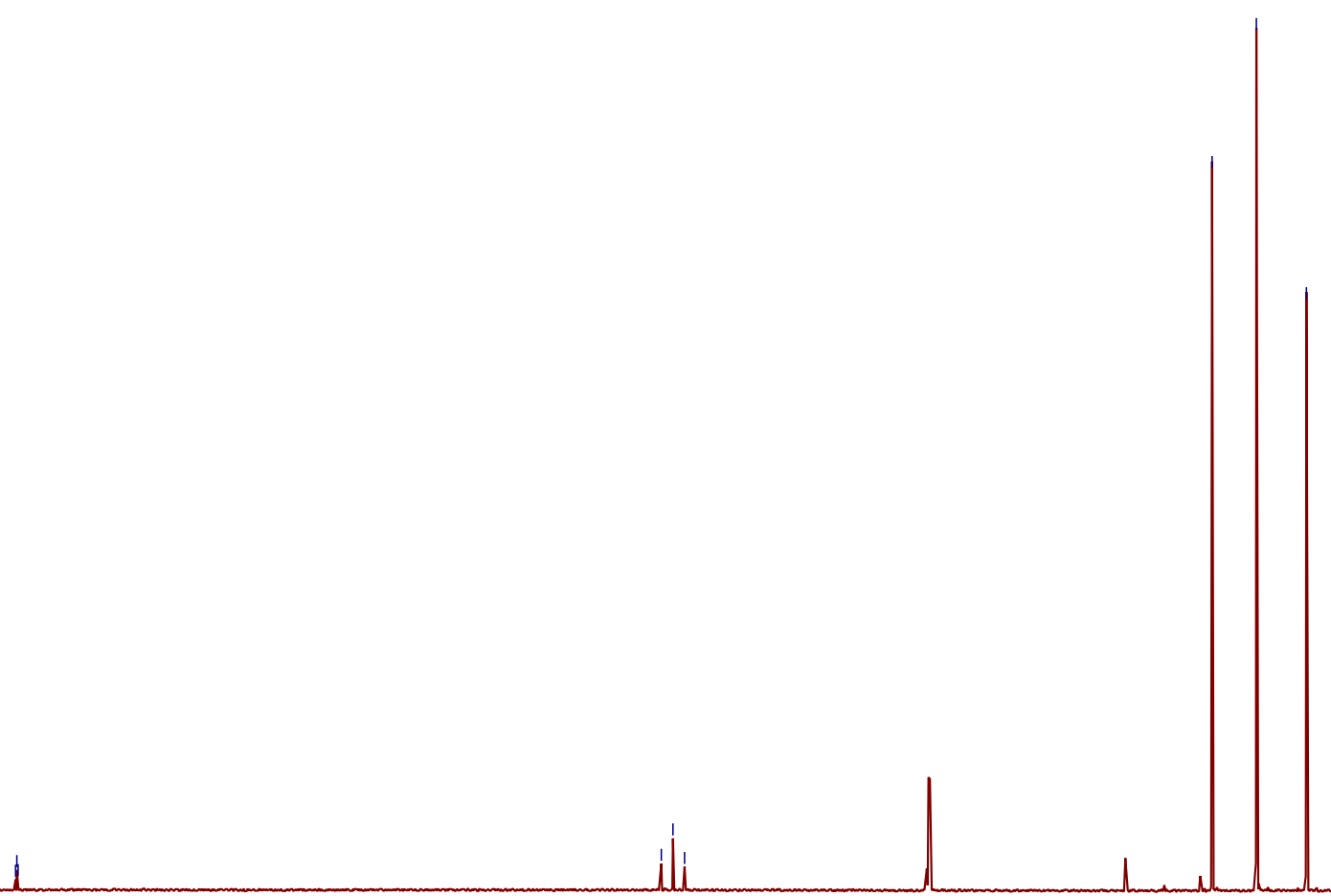

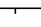

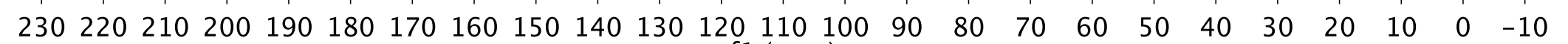
$\mathrm{f} 1(\mathrm{ppm})$ 
<smiles>O=C(SC12CC3CC(CC(C3)C1)C2)C(F)F</smiles>

$1 \mathrm{t}$

${ }^{19}$ F NMR

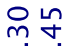

$\stackrel{m}{\sim} \underset{\sim}{\sim}$

긴

nunumum

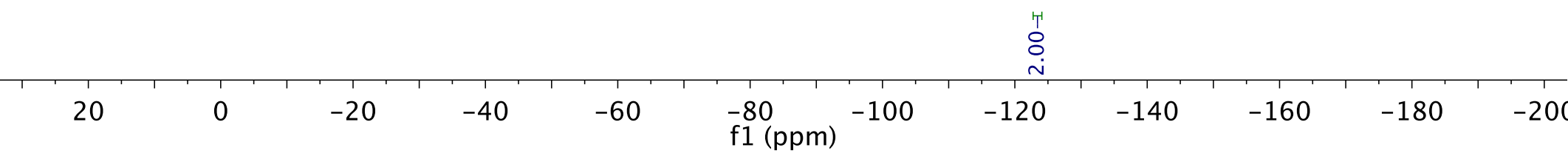


<smiles>COC(C)CCOC(=O)CCSC(=O)C(F)F</smiles>

$1 \mathrm{u}$

${ }^{1} \mathrm{H}$ NMR

กั

نn

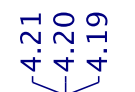

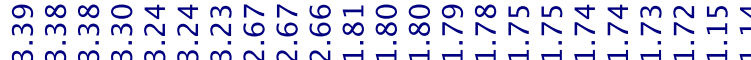

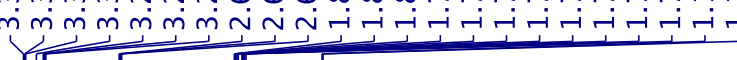

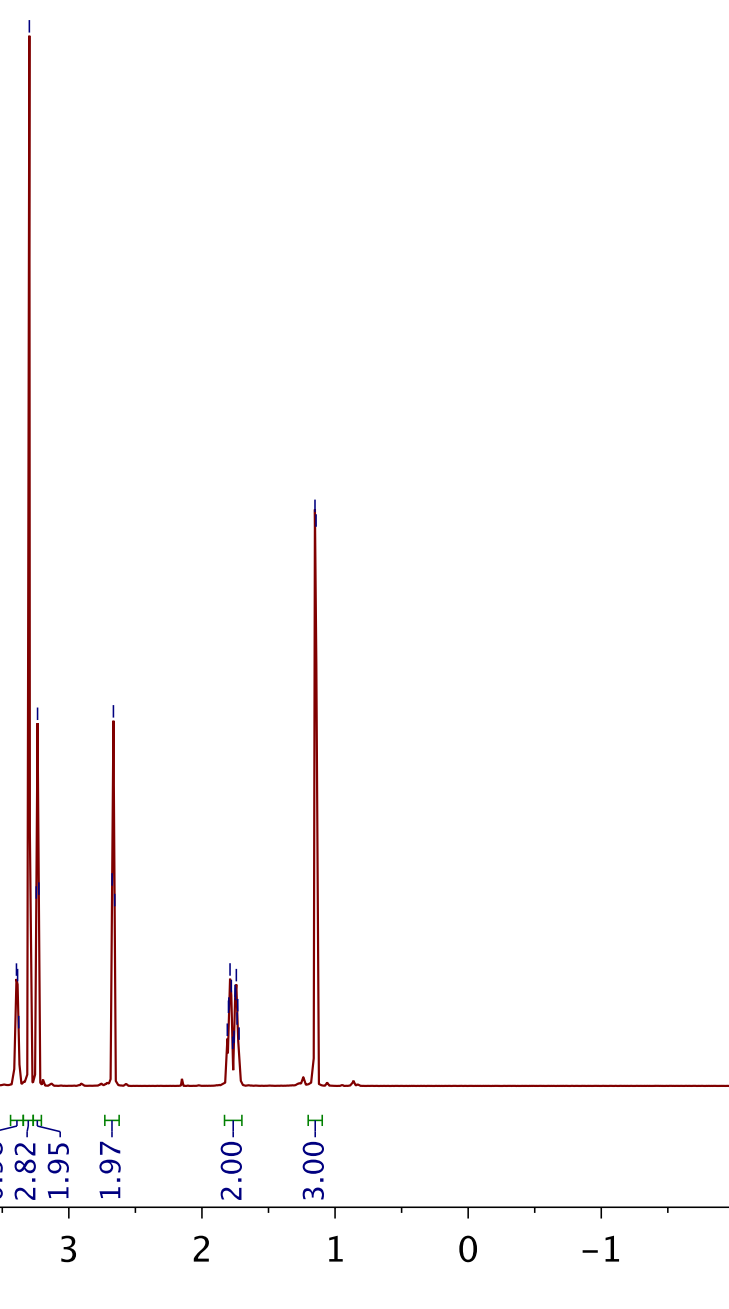


<smiles>COC(C)CCOC(=O)CCSC(=O)C(F)F</smiles>

1u

${ }^{13} \mathrm{C}$ NMR

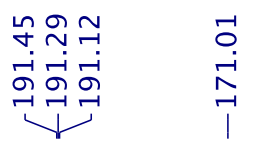

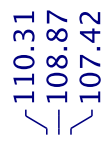

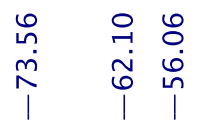

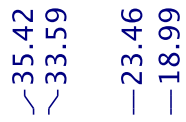

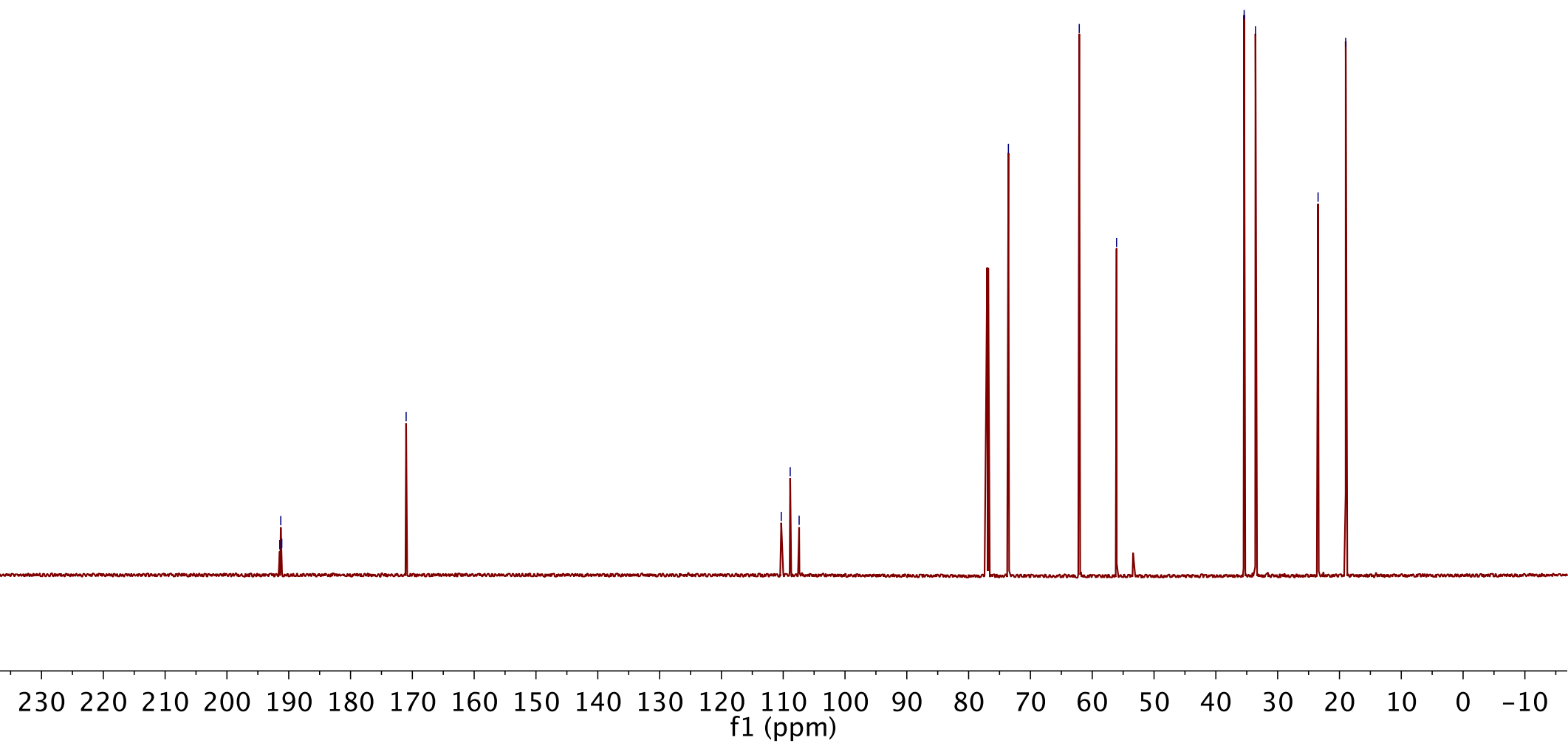


<smiles>COC(C)CCOC(=O)CCSC(=O)C(F)F</smiles>

$1 \mathrm{u}$

${ }^{19} \mathrm{~F}$ NMR

ㄴํㅇ

$\stackrel{m}{\sim}$

$\underset{7}{2}$

V

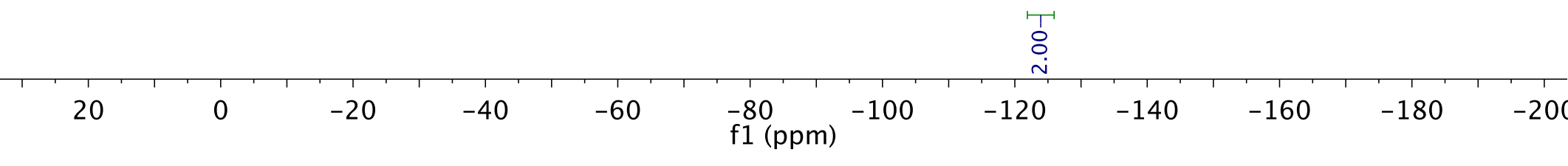


<smiles>COC(=O)C1CCCN1C(=O)[C@H](C)CSC(=O)C(F)F</smiles>

$11 \quad 10$

9

8

7

$\mathrm{f} 1$ (ppm) 
<smiles>COC(=O)C1CCCN1C(=O)[C@H](C)CSC(=O)C(F)F</smiles>

$1 v$

${ }^{13} \mathrm{C}$ NMR

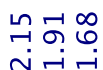

ชี్่̄ं

กำ

in

च

ํํํำ

$0 \infty$

〈।

ิㅡㄲㅇㅛ

inำ

ㄷํㅇํำ

mñ்

r

.

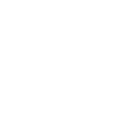


<smiles>COC(=O)C1CCCN1C(=O)[C@H](C)CSC(=O)C(F)F</smiles>

1v

${ }^{19} \mathrm{~F}$ NMR

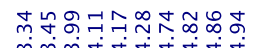



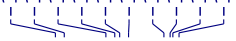

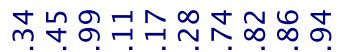

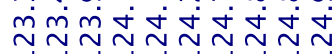
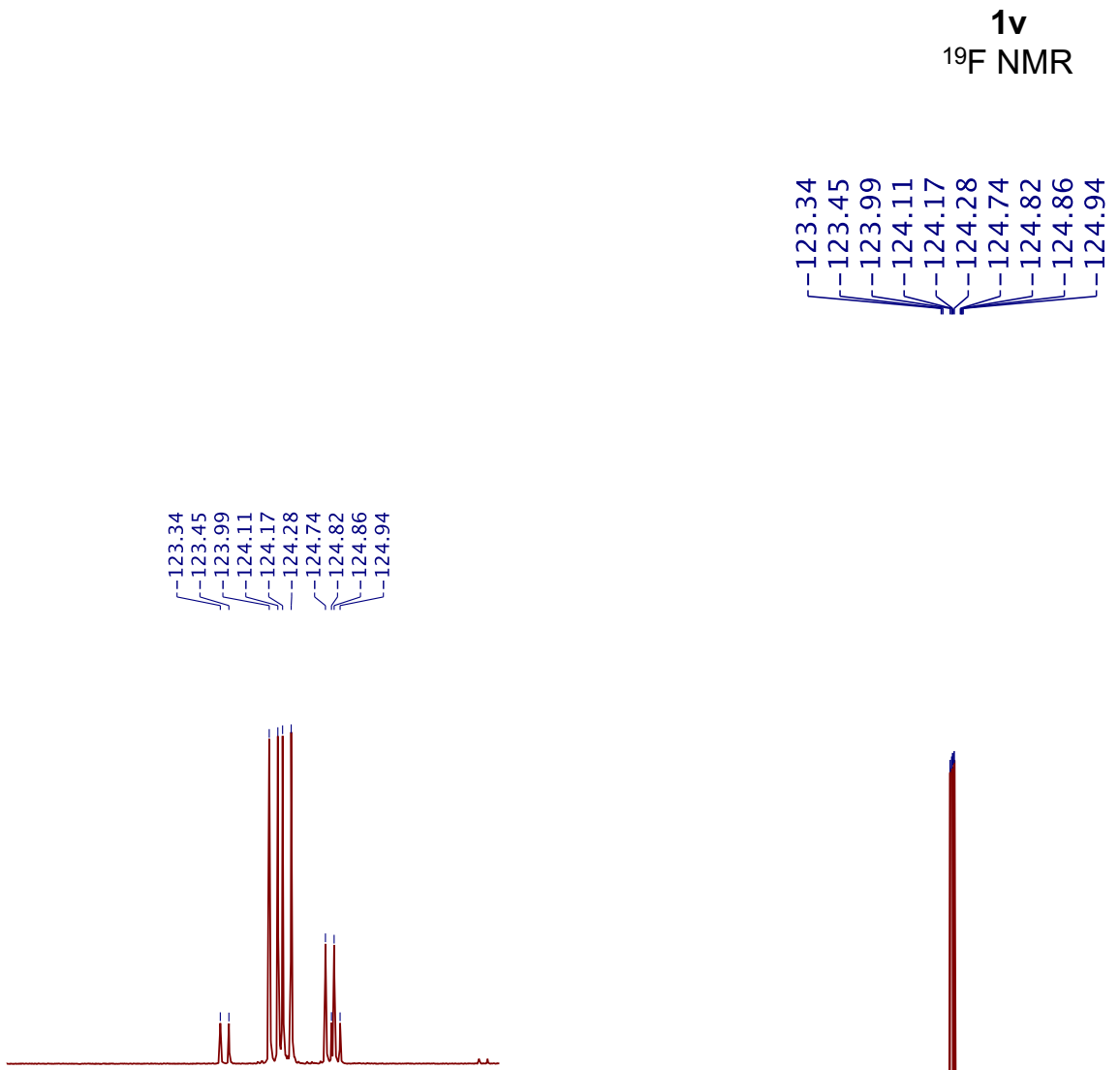

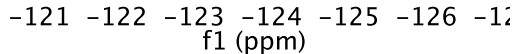

\begin{tabular}{llllllllllllllll}
\hline-60 & -70 & -80 & -90 & -100 & -110 & -120 & -130 & -140 & -150 & -160 & -170 & -180 & -190 & $-2(\mathrm{ppm})$ &
\end{tabular}



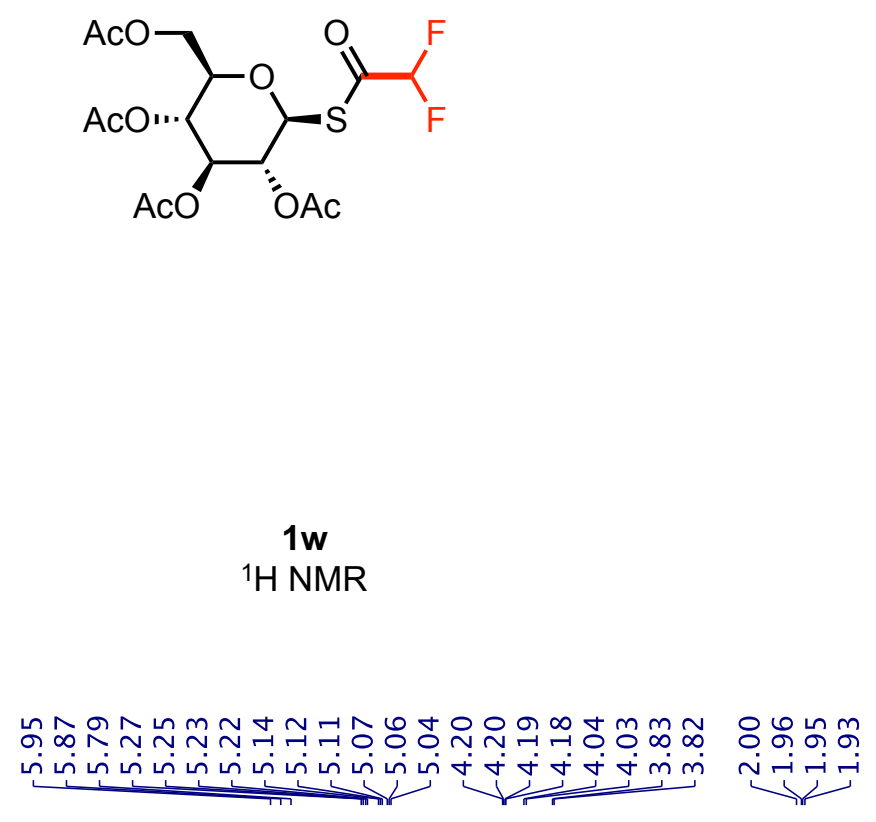

$1 w$

ดุ

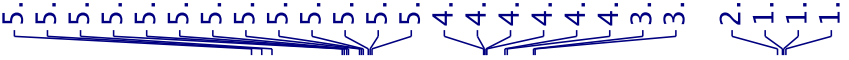

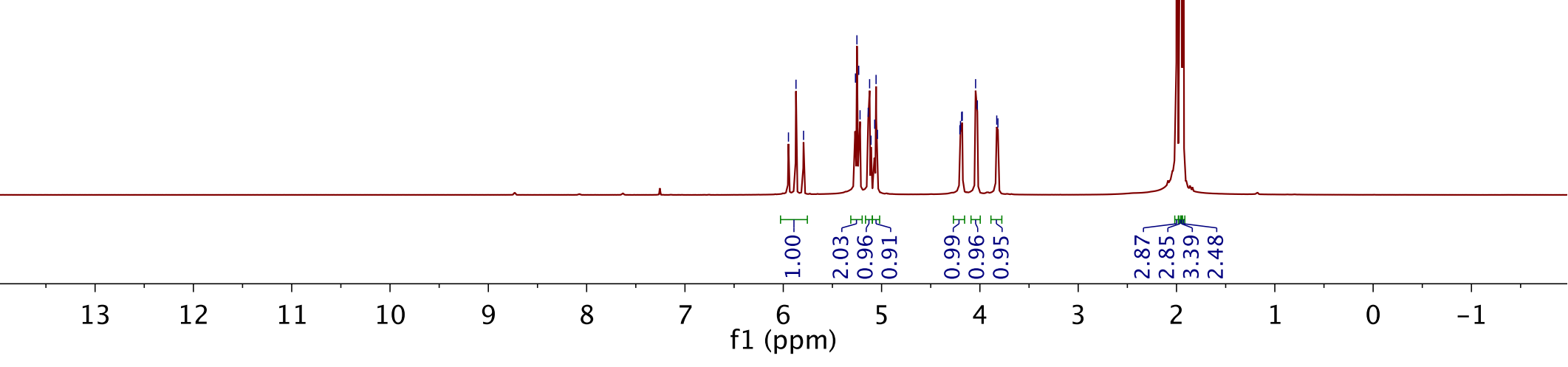




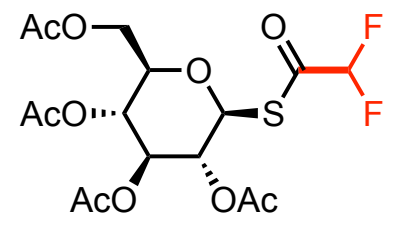

$\stackrel{1 w}{{ }^{13} \text { C NMR }}$

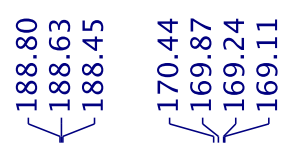

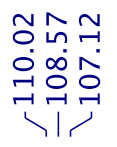

근 ㄴํํำ

शंर्रण0்

रा?

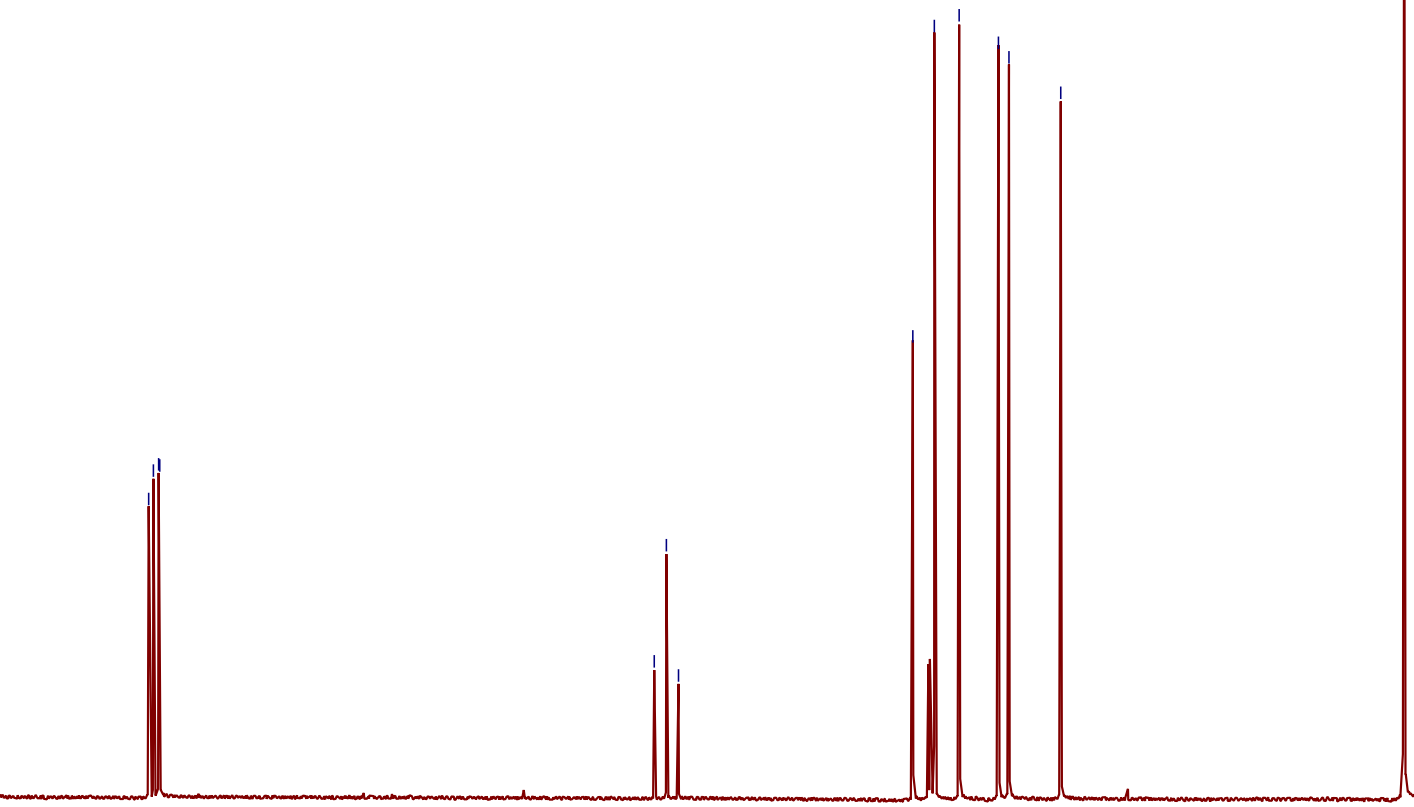

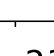

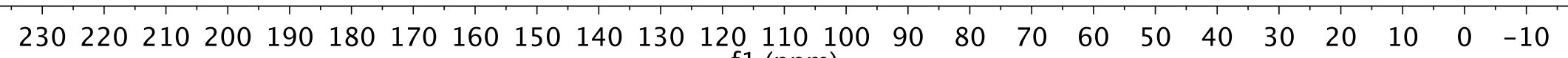
f1 (ppm) 


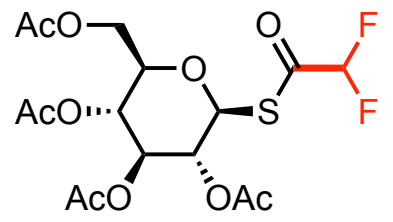

$1 w$

${ }^{19} \mathrm{~F}$ NMR

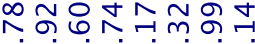

$\approx \approx m m \dot{\sim}+\omega^{\prime}$

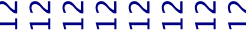

(1)

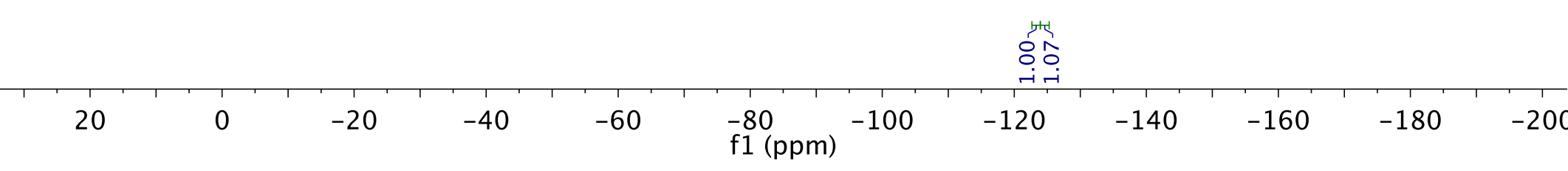




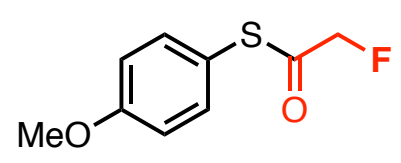

$1 \mathrm{x}$

${ }^{1} \mathrm{H}$ NMR

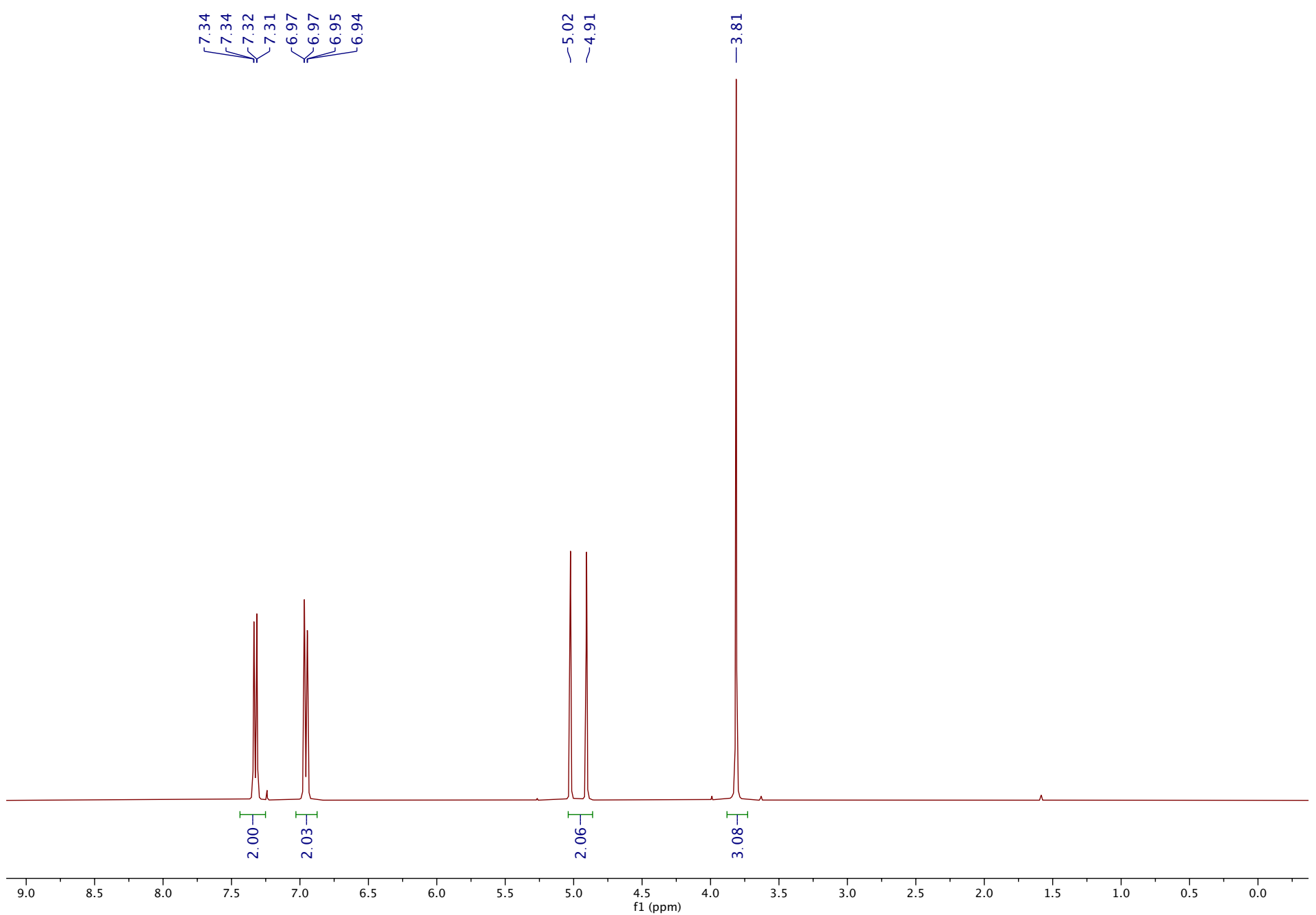



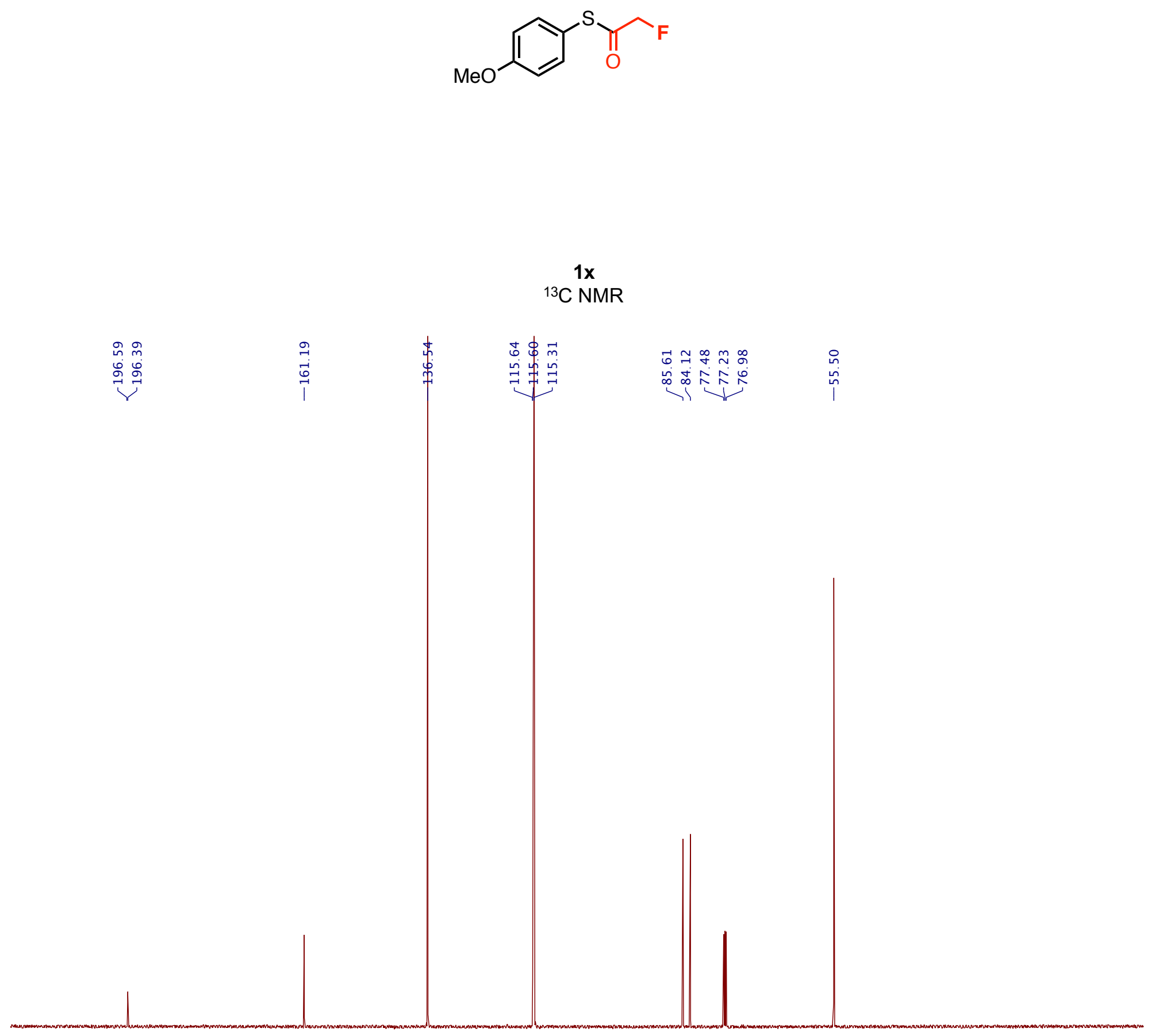


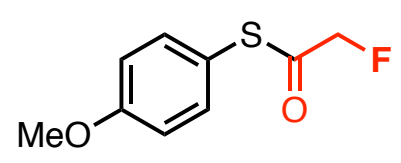

$1 \mathrm{x}$

${ }^{19} \mathrm{~F}$ NMR

앙요줏

$\stackrel{\sim}{\sim} \stackrel{\sim}{\sim} \stackrel{\sim}{\sim}$

$>1$

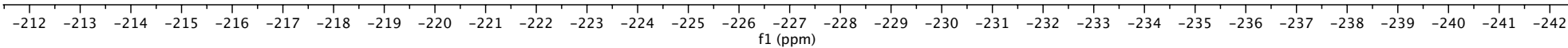




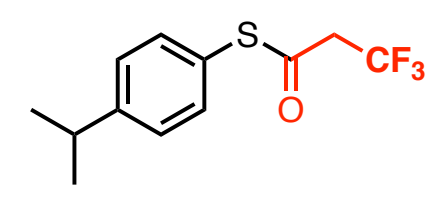

$1 y$

${ }^{1} \mathrm{H}$ NMR

$\hat{m} \stackrel{m}{m} \ddot{m}$

수솟

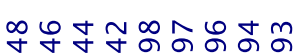

minnminivini
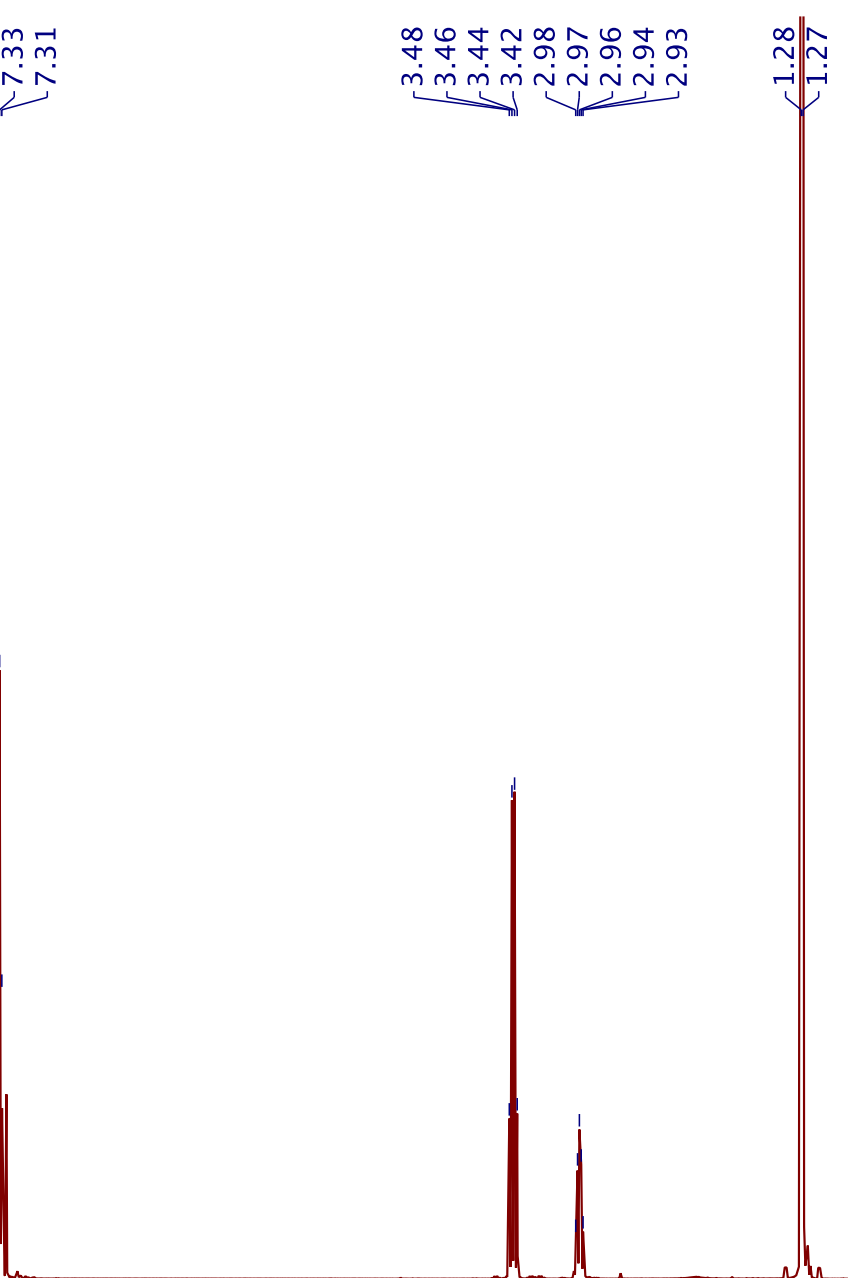

인

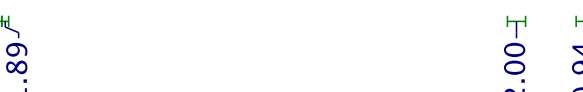

क़

$\begin{array}{llllll}13 & 12 & 11 & 10 & 9 & 8\end{array}$

$8 \quad 7 \quad 6$

f1 (ppm)

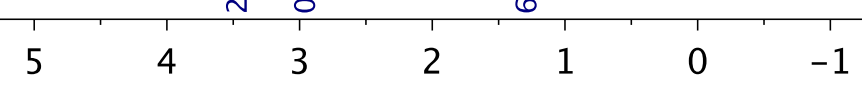


<smiles>CC(C)c1ccc(SC(=O)CC(F)(F)F)cc1</smiles>

$1 y$

${ }^{13} \mathrm{C}$ NMR

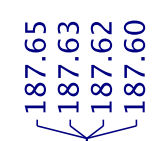

$\stackrel{\infty}{\sim}$

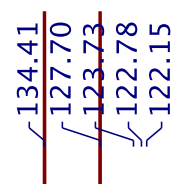

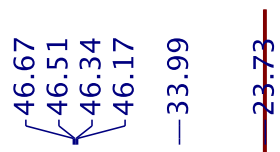

r

$\stackrel{\text { 는 }}{r}$

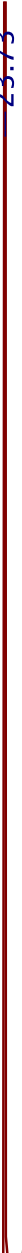

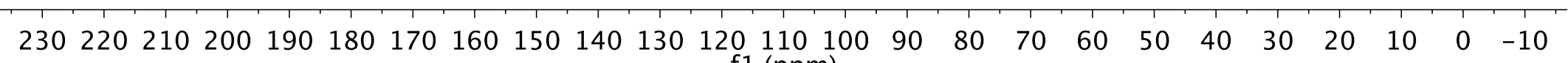
f1 (ppm) 


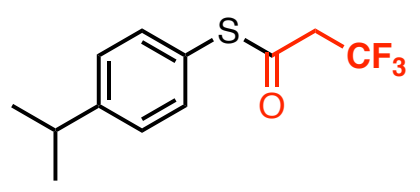

$1 y$

${ }^{19} \mathrm{~F}$ NMR

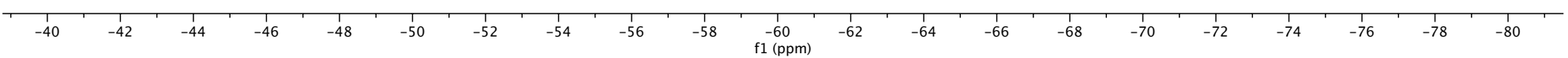


<smiles>COc1ccc(SC(=O)C(F)C(F)(F)F)cc1</smiles>

$1 z$

${ }^{19} \mathrm{~F}$ NMR
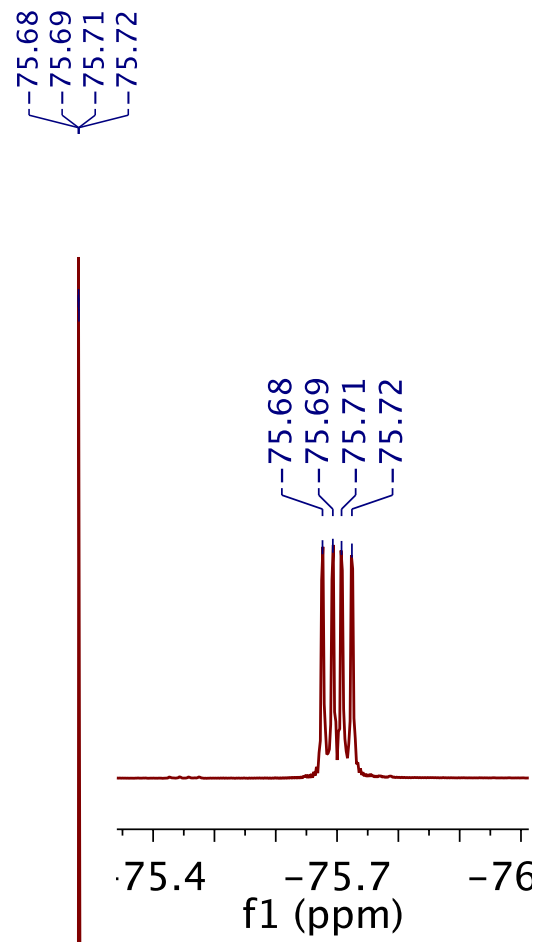

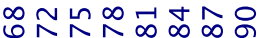

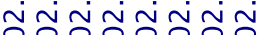
กNNNNNำ

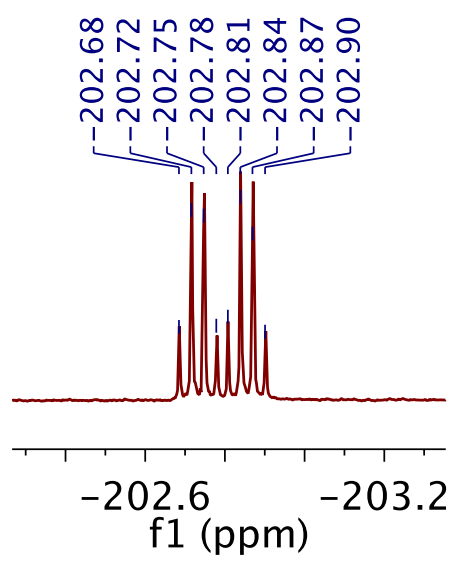

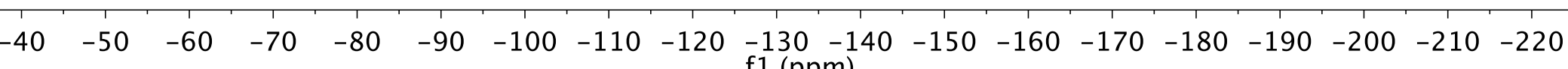
f1 (ppm) 
<smiles>COc1ccc(SC(=O)C(F)(F)c2ccccc2)cc1</smiles>

1 aa

${ }^{1} \mathrm{H}$ NMR

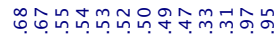

reninivivio

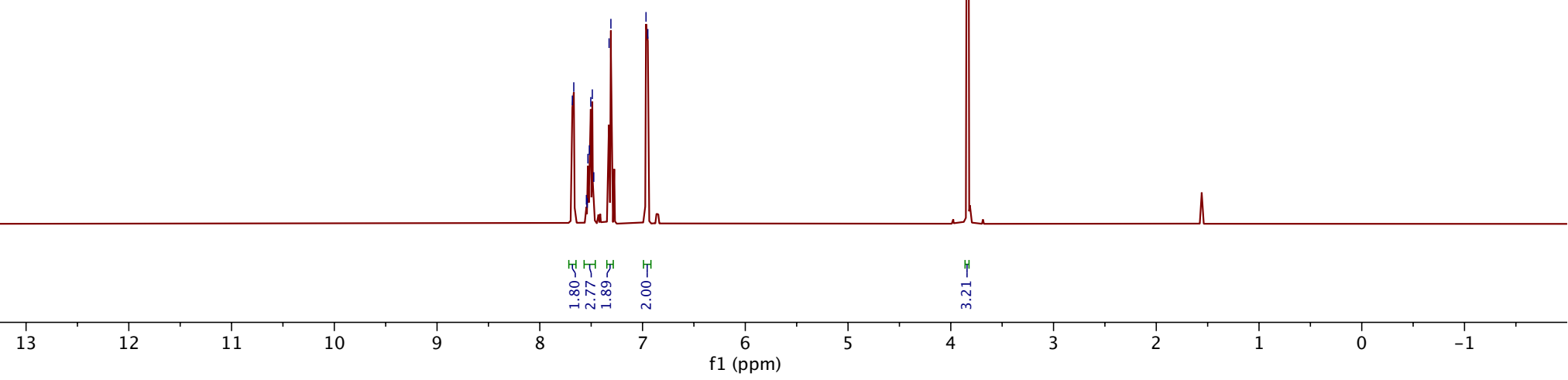


<smiles>COc1ccc(SC(=O)C(F)(F)c2ccccc2)cc1</smiles>

1 aa

${ }^{13} \mathrm{C}$ NMR
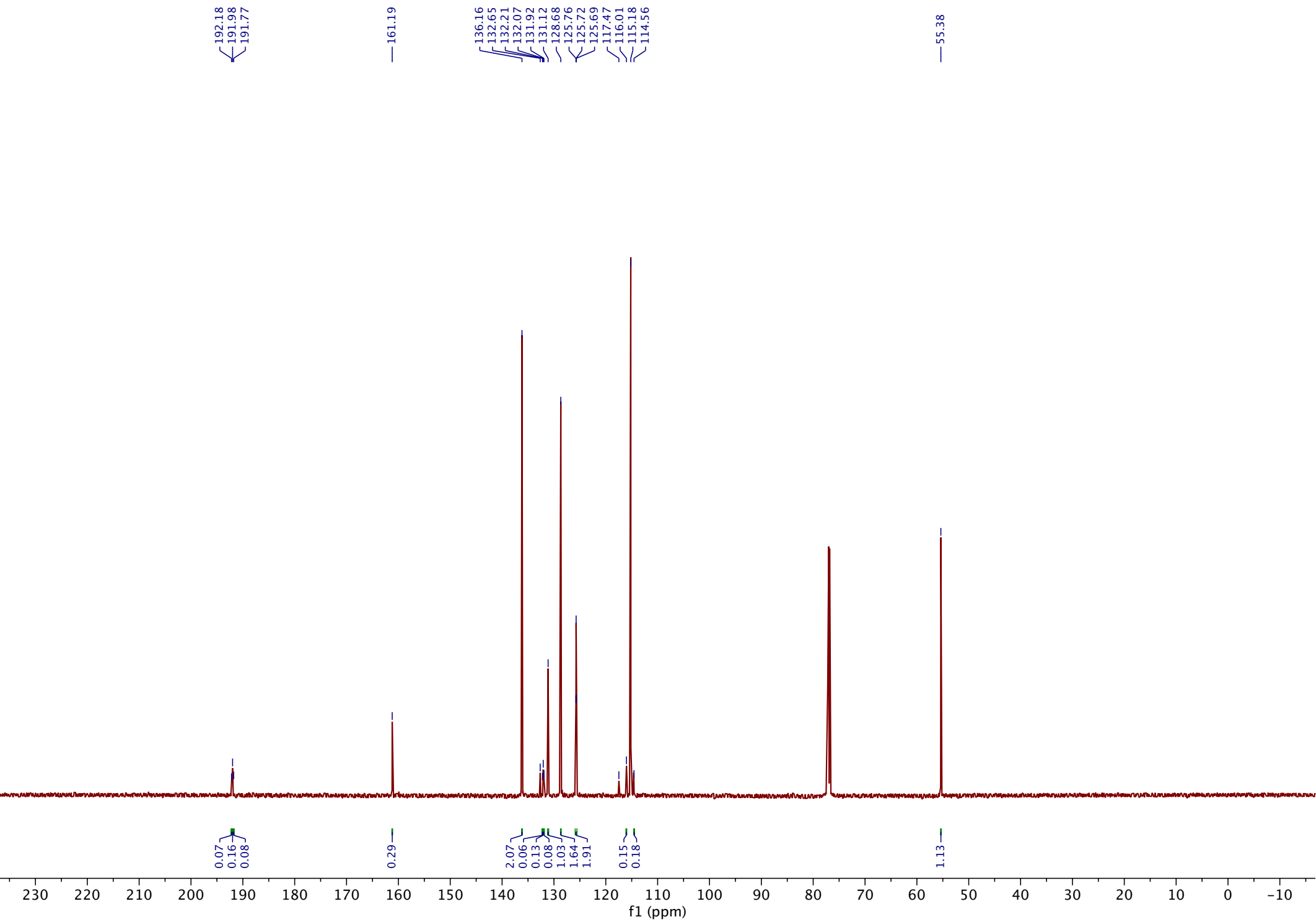
<smiles>COc1ccc(SC(=O)C(F)(F)c2ccccc2)cc1</smiles>

1 aa

${ }^{19} \mathrm{~F}$ NMR

ì

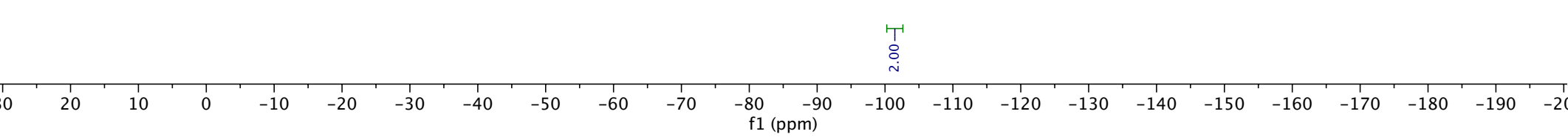




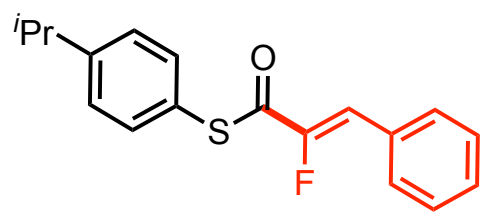

$1 \mathrm{ab}$

$1 \mathrm{H} N M R$

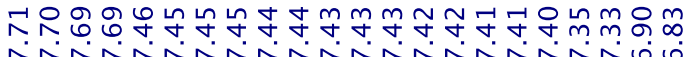

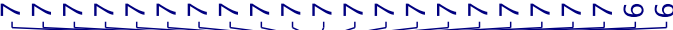

ㅇํำดัดูก

minn
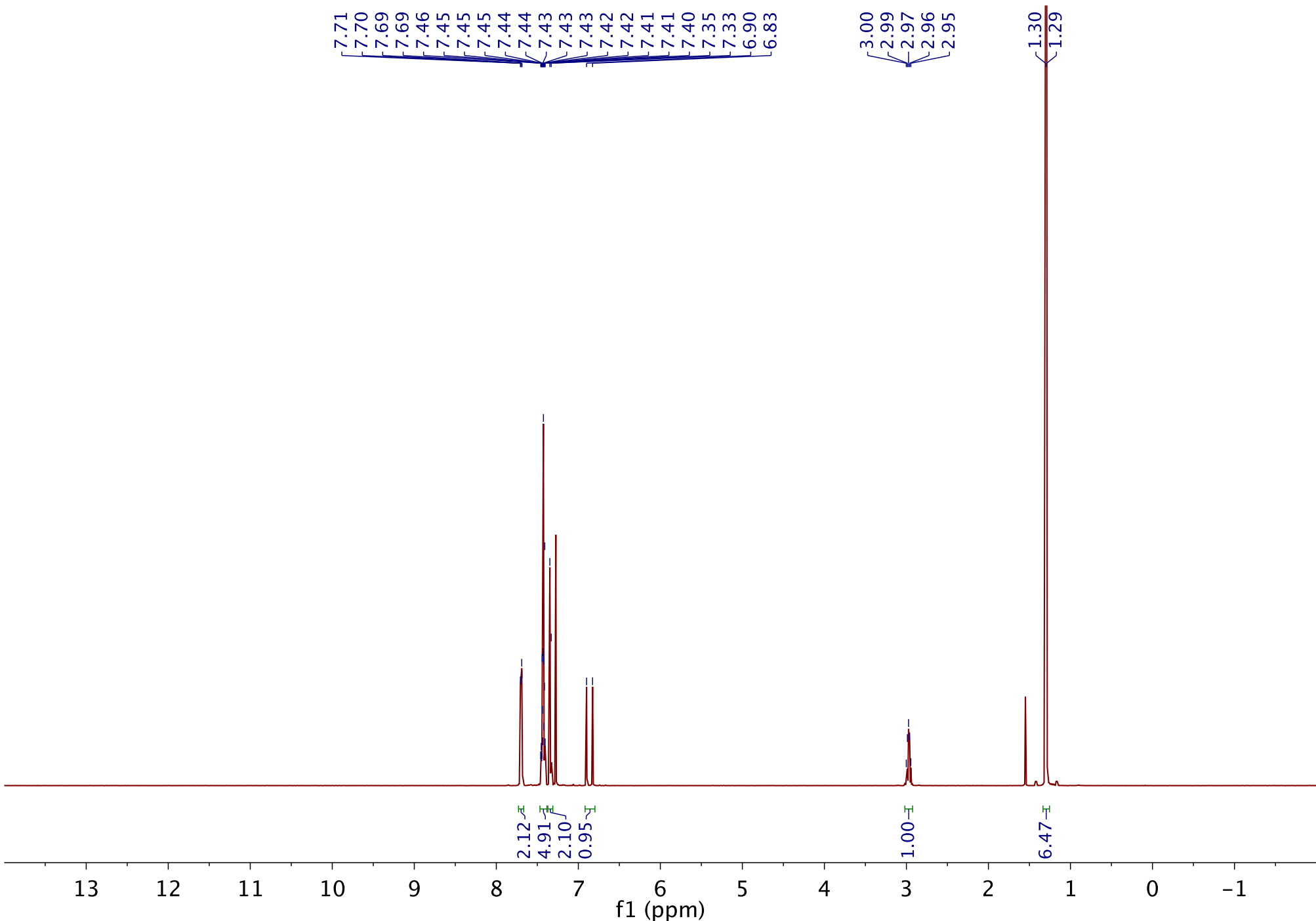


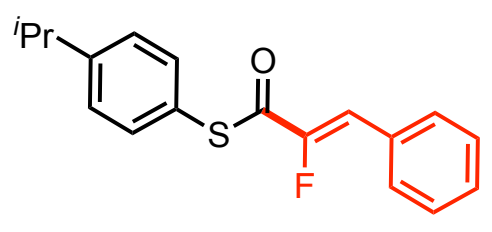

$1 a b$

${ }^{13} \mathrm{C}$ NMR

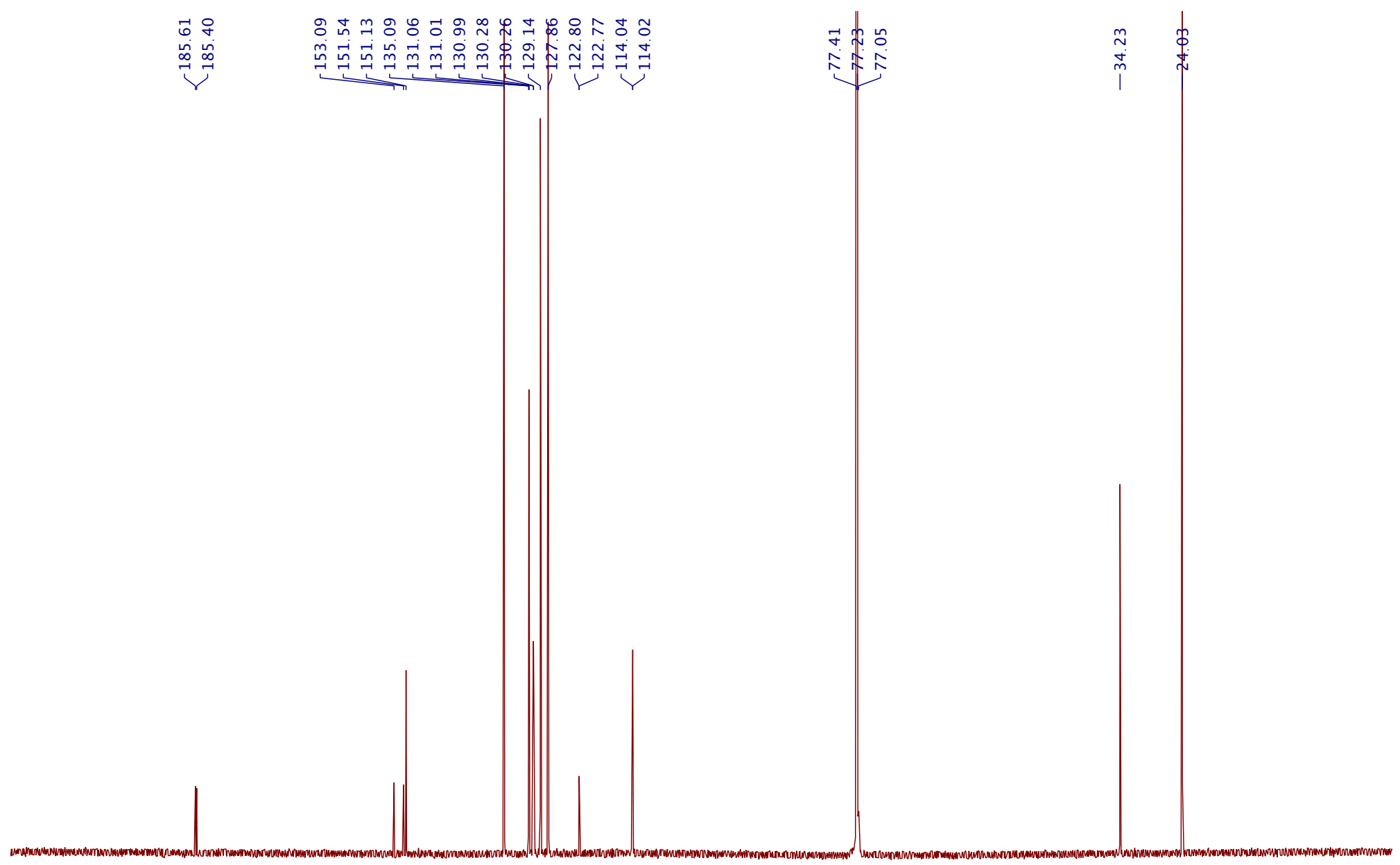

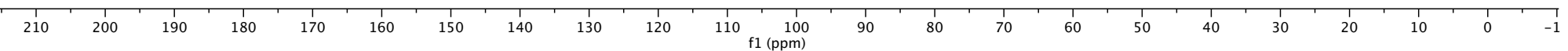




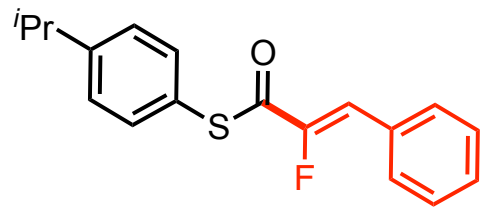

$1 a b$

${ }^{19} \mathrm{~F}$ NMR

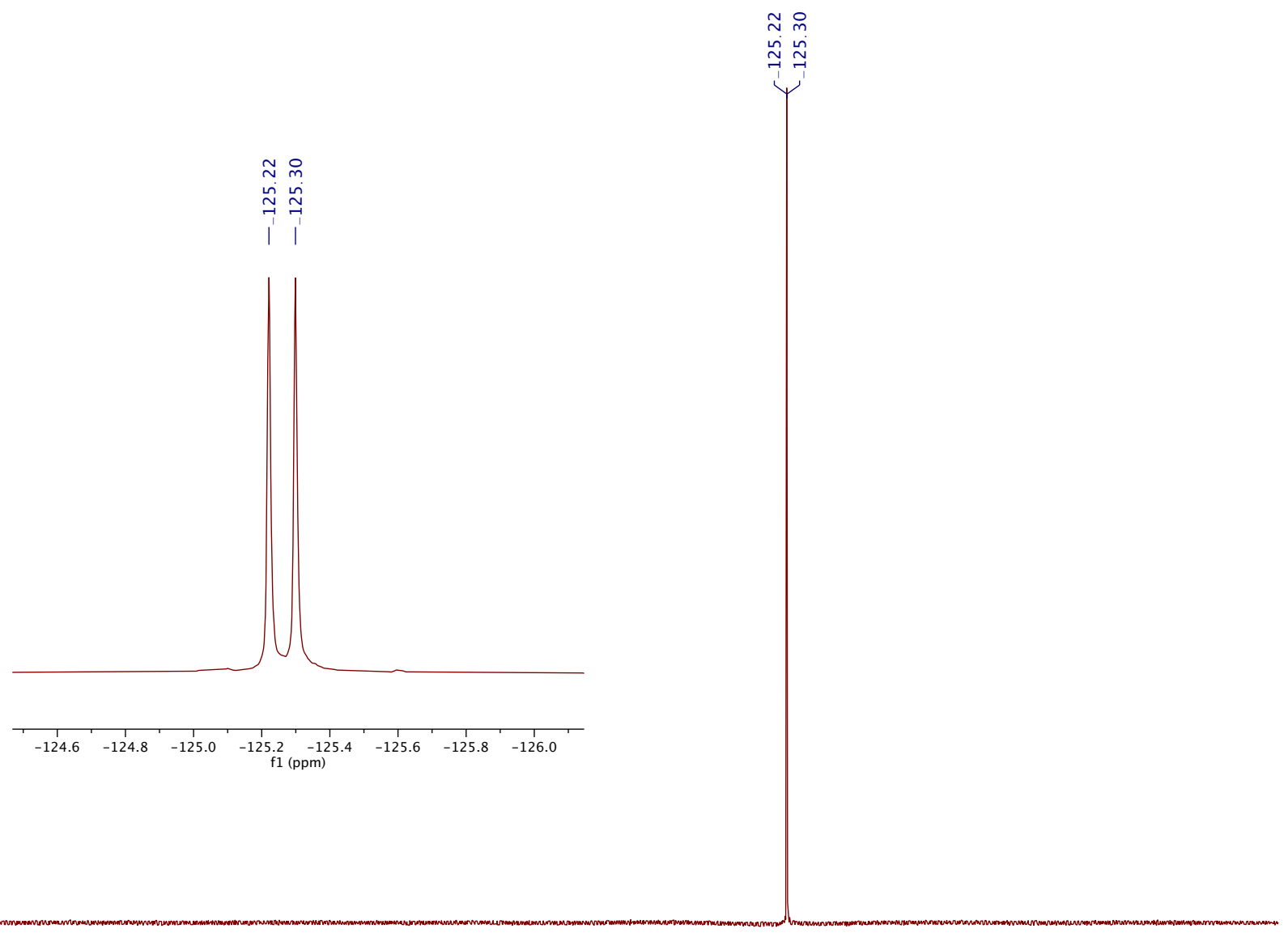

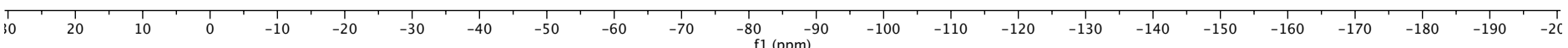




\section{T)}

$2 a$

${ }^{1} \mathrm{H}$ NMR

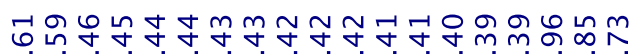

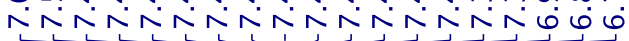

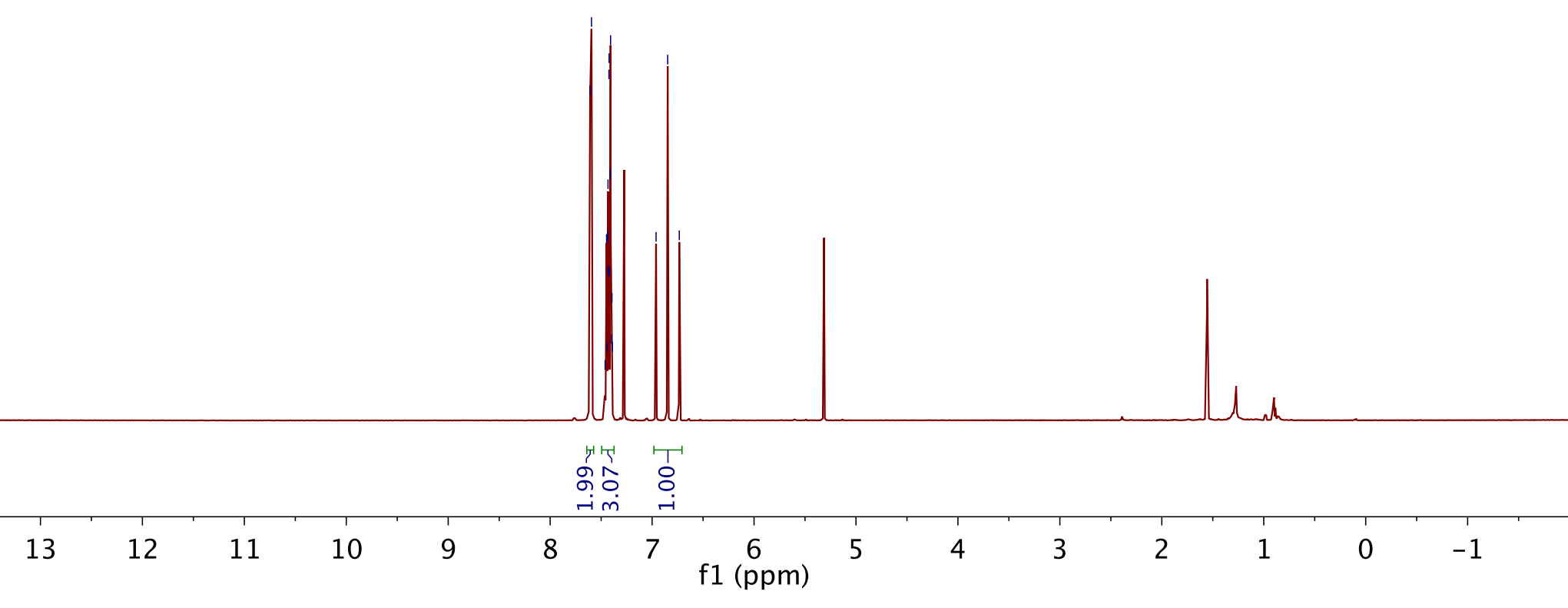


<smiles>[AlH2]</smiles>

2a

${ }^{13} \mathrm{C}$ NMR

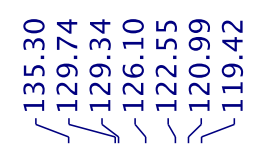

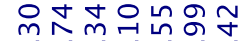

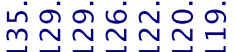

武门17

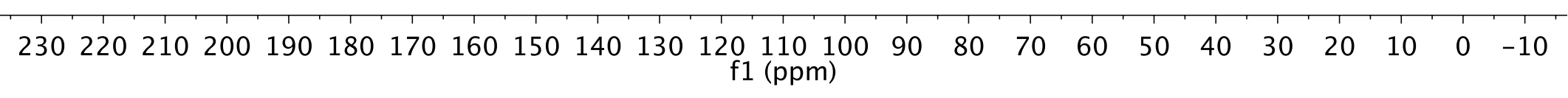


<smiles>COc1ccc(SC(F)F)cc1</smiles>

2b

${ }^{1} \mathrm{H}$ NMR

ผีำสㄷㅀ숭

ก

$\infty$

人

$m$

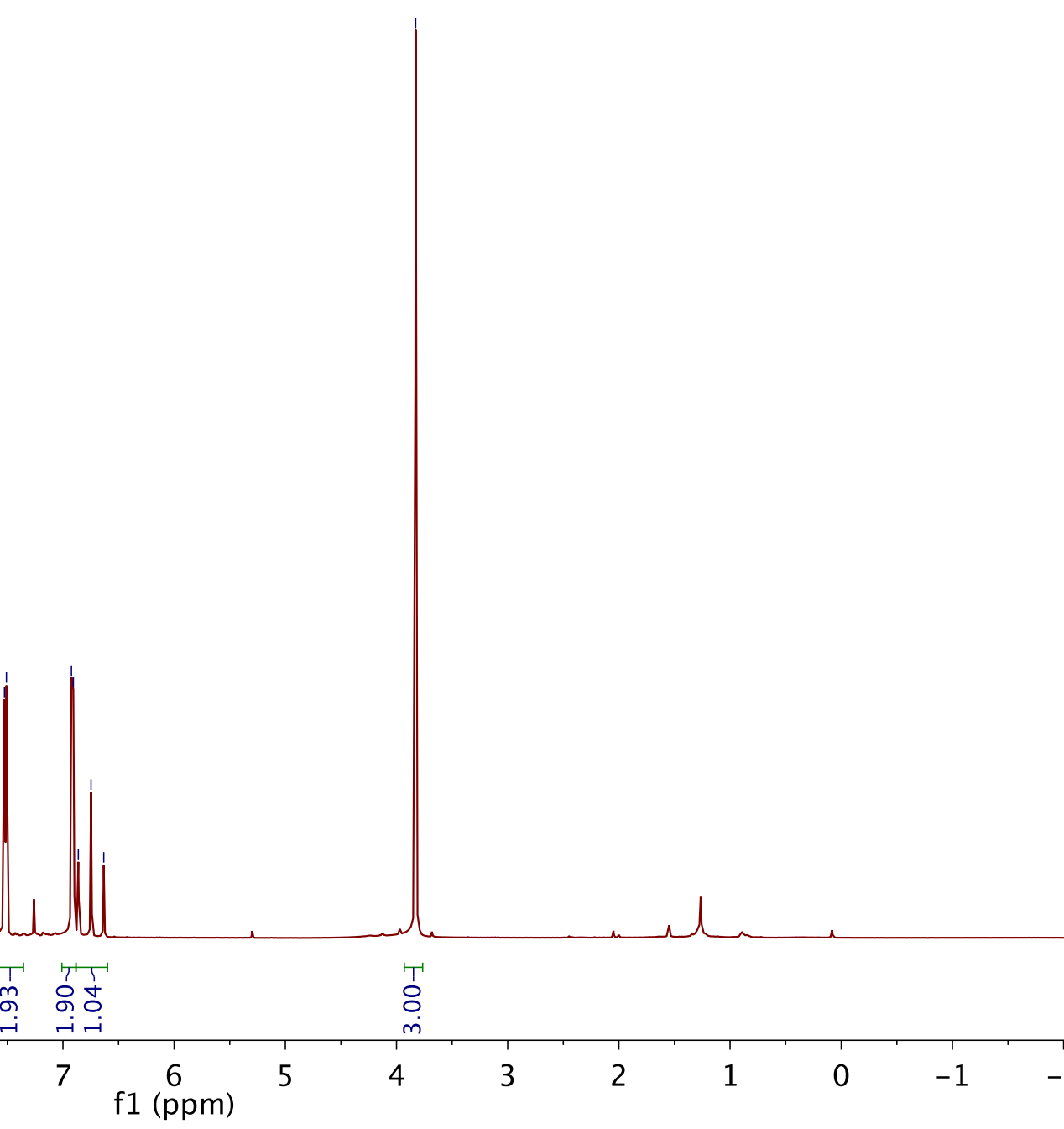


<smiles>COc1ccc(SC(F)F)cc1</smiles>

2b

${ }^{19} \mathrm{~F}$ NMR

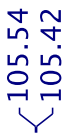

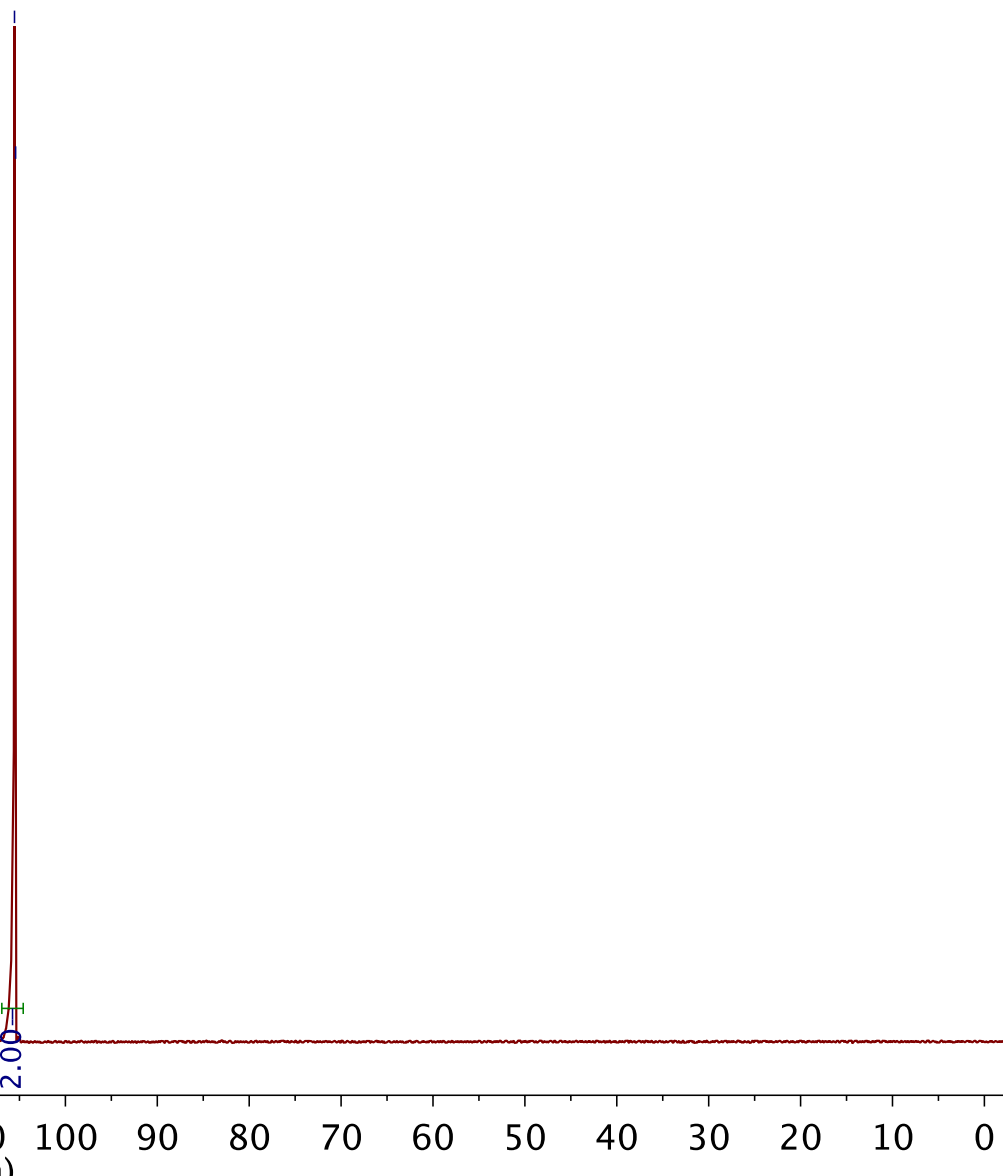




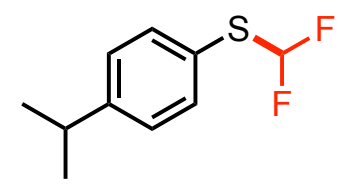

2c

1 H NMR

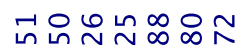

NNÑ்
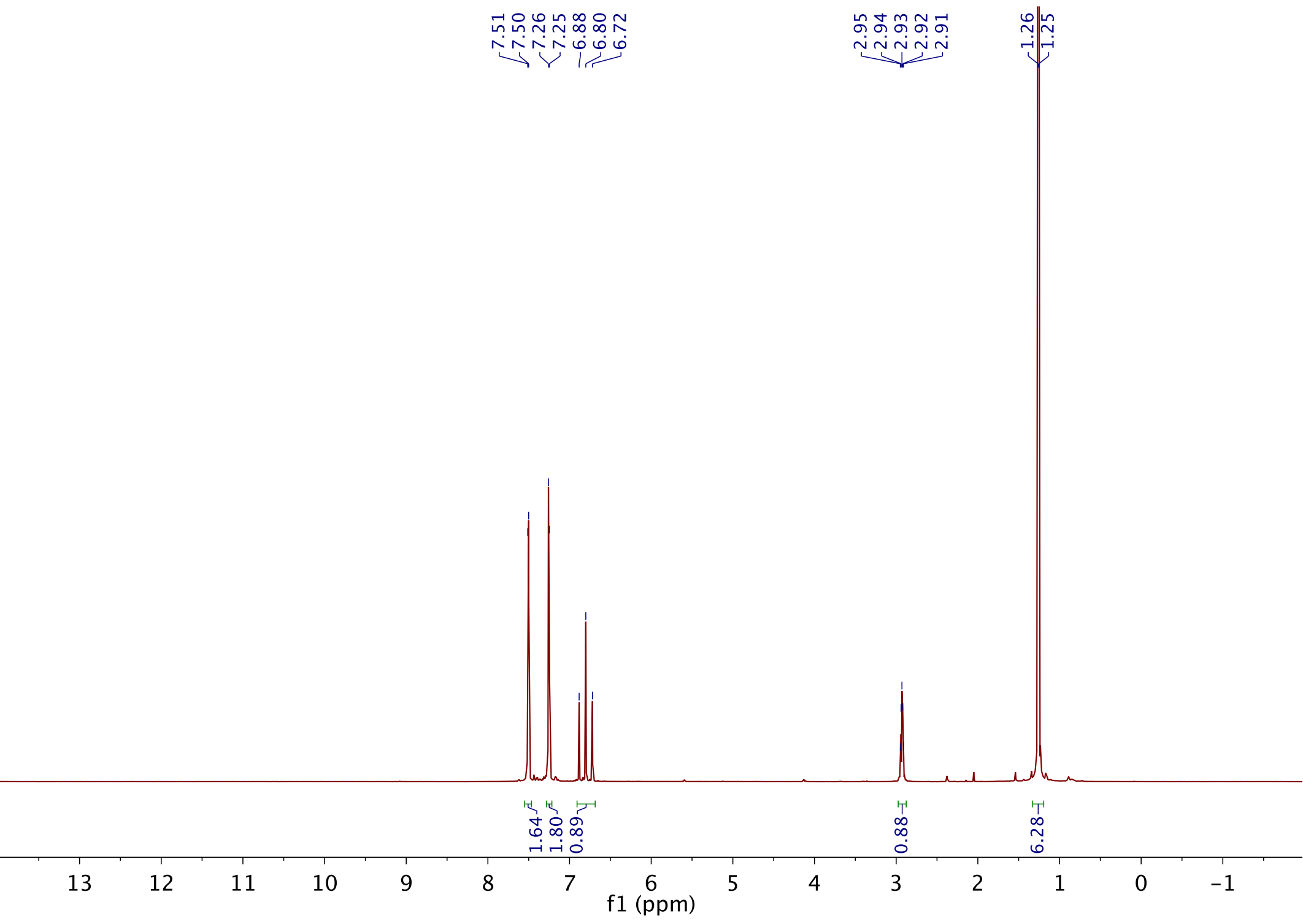


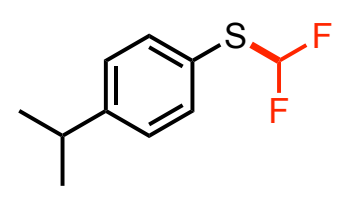

2c

${ }^{13} \mathrm{C}$ NMR

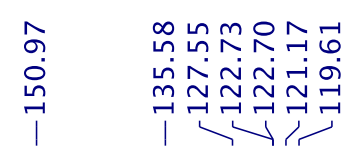

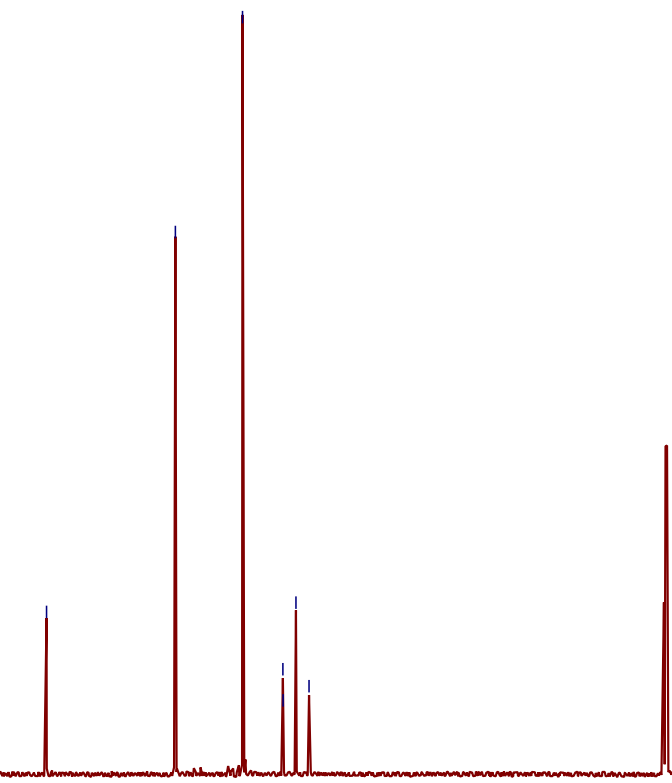

$$
+
$$




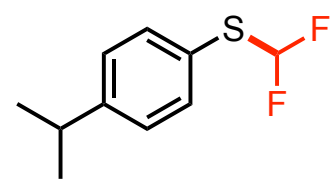

2c

${ }^{19} \mathrm{~F}$ NMR

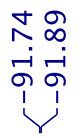

\begin{tabular}{|c|c|c|c|c|c|c|c|c|c|c|c|}
\hline & & & & & $\begin{array}{l}1 \\
8 \\
\dot{i}\end{array}$ & & & & & & \\
\hline 20 & 0 & -20 & -40 & -60 & $\begin{array}{l}-80 \\
\mathrm{f} 1(\mathrm{ppm})\end{array}$ & -100 & -120 & -140 & -160 & -180 & -200 \\
\hline
\end{tabular}




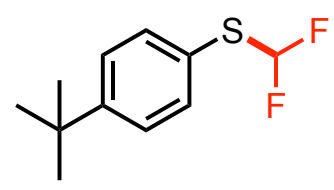

2d

${ }^{1} \mathrm{H}$ NMR

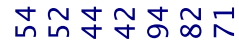

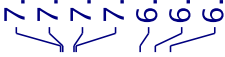

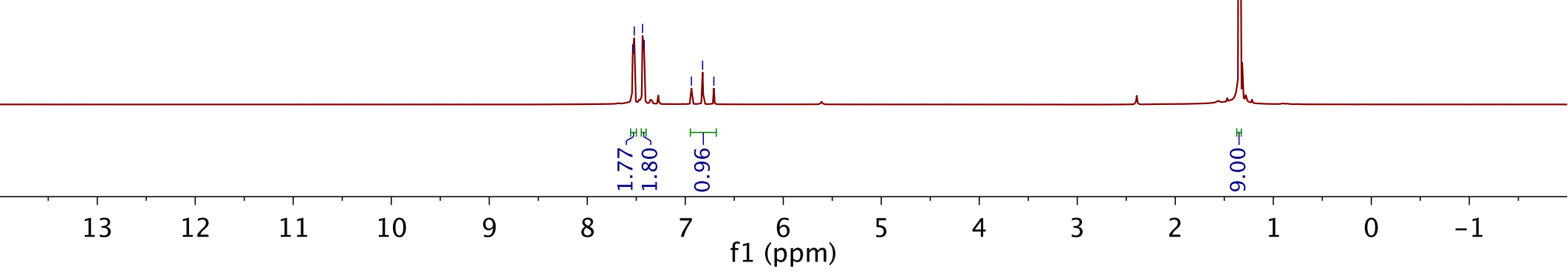




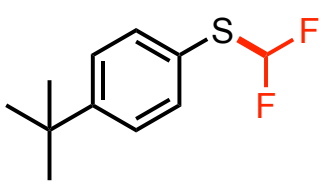

2d

${ }^{13} \mathrm{C}$ NMR

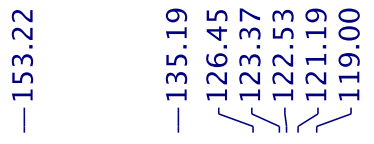

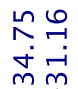

n $m \sim \pi \sigma$

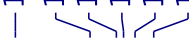

23 


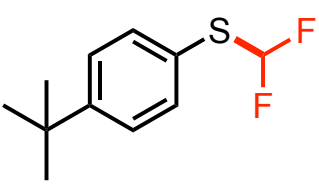

2d

${ }^{19} \mathrm{~F}$ NMR

$$
\begin{aligned}
& \pi \infty \\
& \text { क्ष }
\end{aligned}
$$

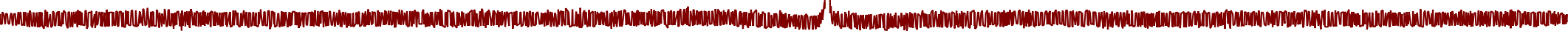

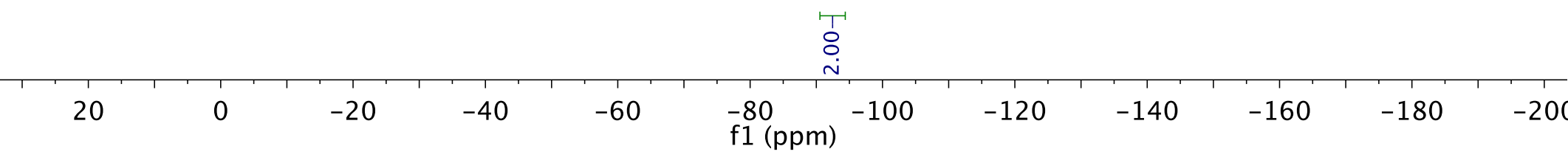




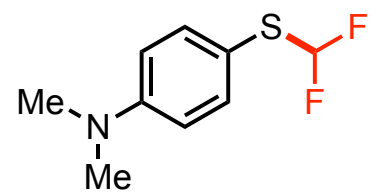

$2 e$

${ }^{1} \mathrm{H}$ NMR

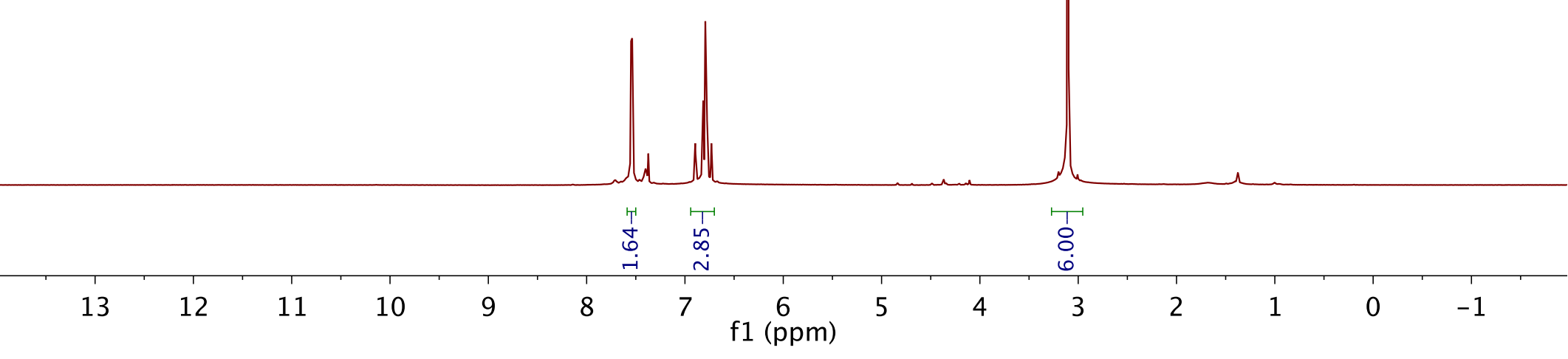


<smiles>CN(C)c1ccc(SC(F)F)cc1</smiles>

$2 e$

${ }^{13} \mathrm{C}$ NMR

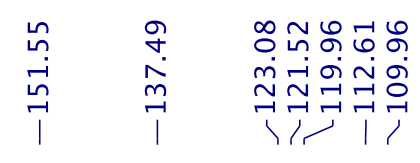

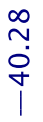

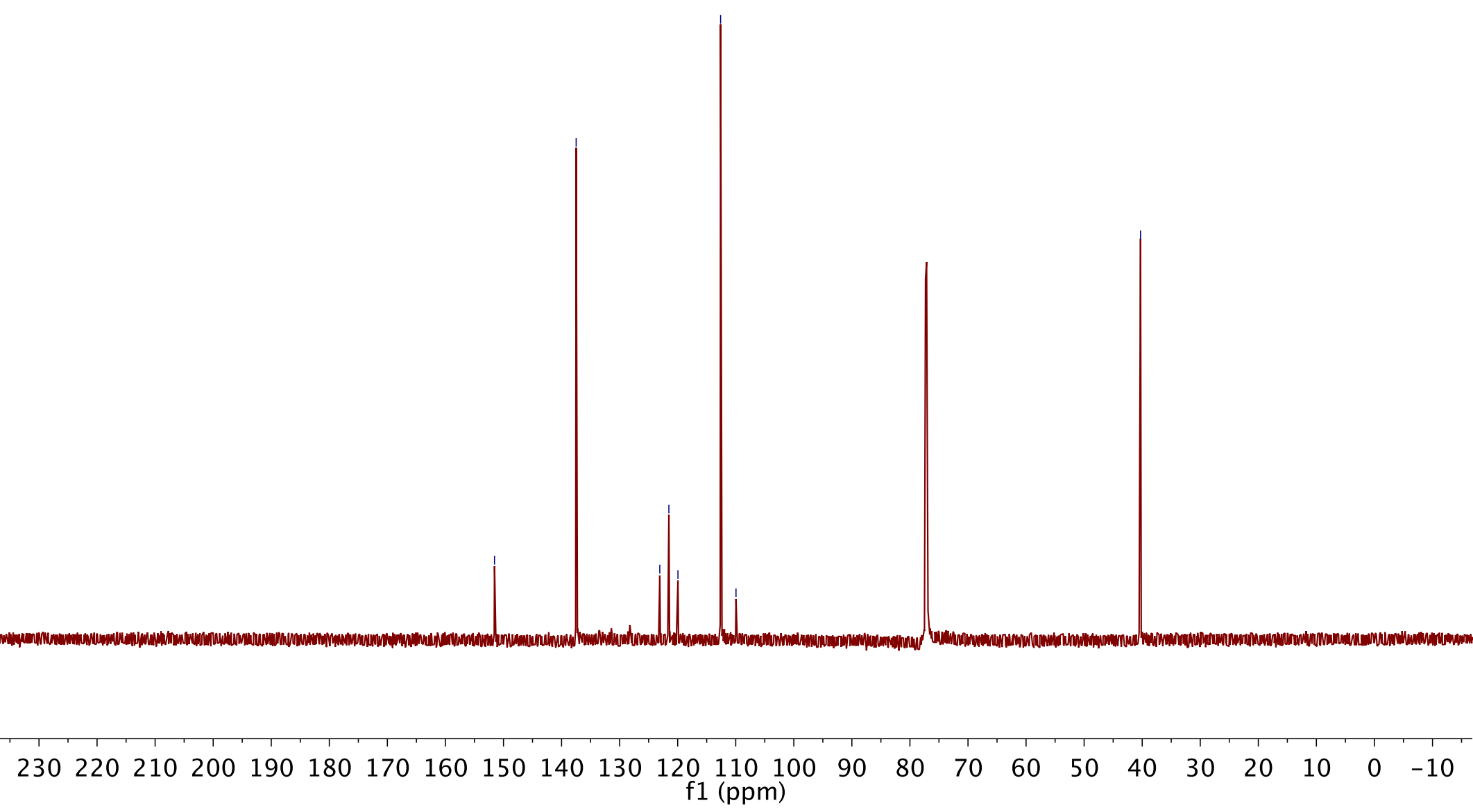




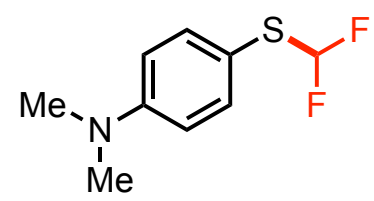

$2 e$

${ }^{19} \mathrm{~F} \mathrm{NMR}$

กำ

สี่ั่

की

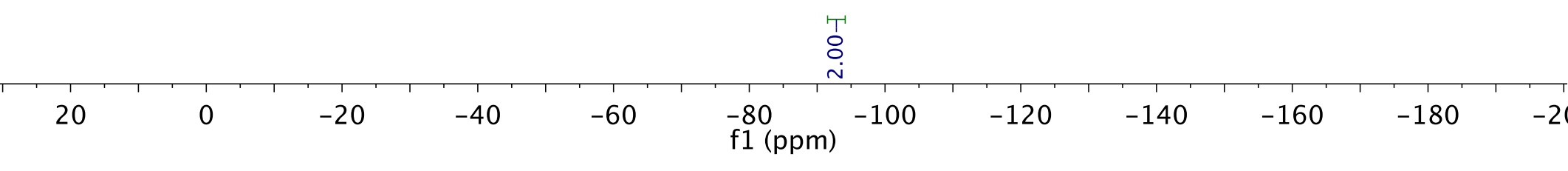




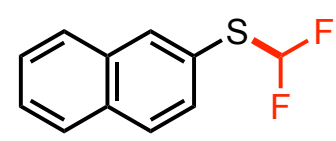

$2 f$

${ }^{1} \mathrm{H}$ NMR

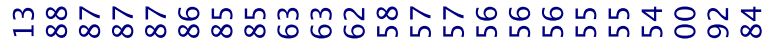

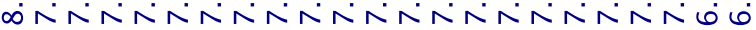

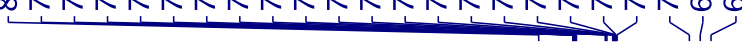


<smiles>[AlH2]</smiles>

$2 f$

${ }^{13} \mathrm{C}$ NMR

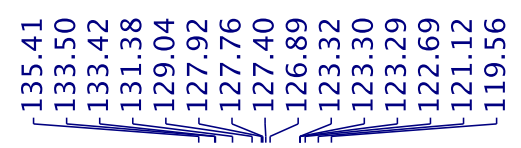

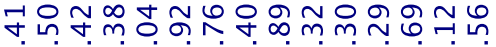

n்mं

政

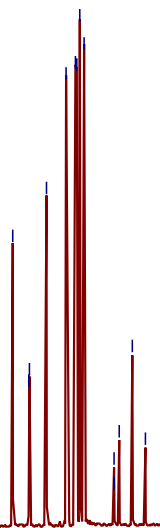

$\begin{array}{cccccccccccccccccccc}230 & 220 & 210 & 200 & 190 & 180 & 170 & 160 & 150 & 140 & 130 & 120 \\ \mathrm{f} 1(\mathrm{ppm}) & 110 & 100 & 90 & 80 & 70 & 60 & 50 & 40 & 30 & 20 & 10 & 0 & -10\end{array}$


<smiles>FC(F)Sc1ccc2ccccc2c1</smiles>

도 6

नंबू

I

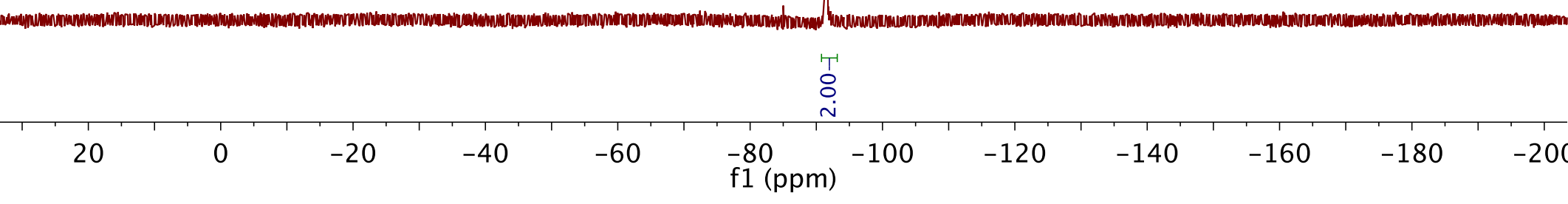


<smiles>O=C(Nc1ccc(SC(F)F)cc1)C(F)(F)F</smiles>

2i

${ }^{1} \mathrm{H}$ NMR

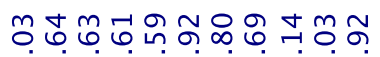

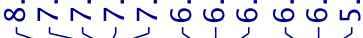

l?

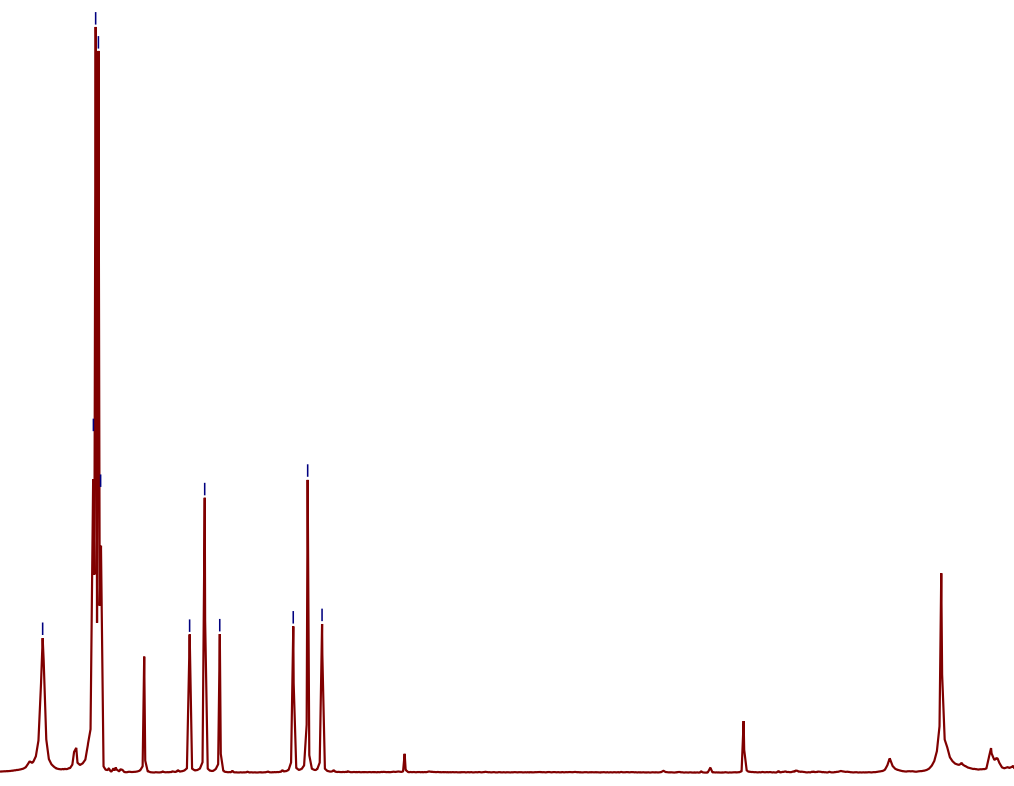

$\begin{array}{lllll}13 & 12 & 11 & 10 & 9\end{array}$

8

7

f1 $\left(\begin{array}{l}6 \\ \mathrm{ppm})\end{array}\right.$ 
<smiles>O=C(Nc1ccc(SC(F)F)cc1)C(F)(F)F</smiles>

2i

${ }^{13} \mathrm{C}$ NMR
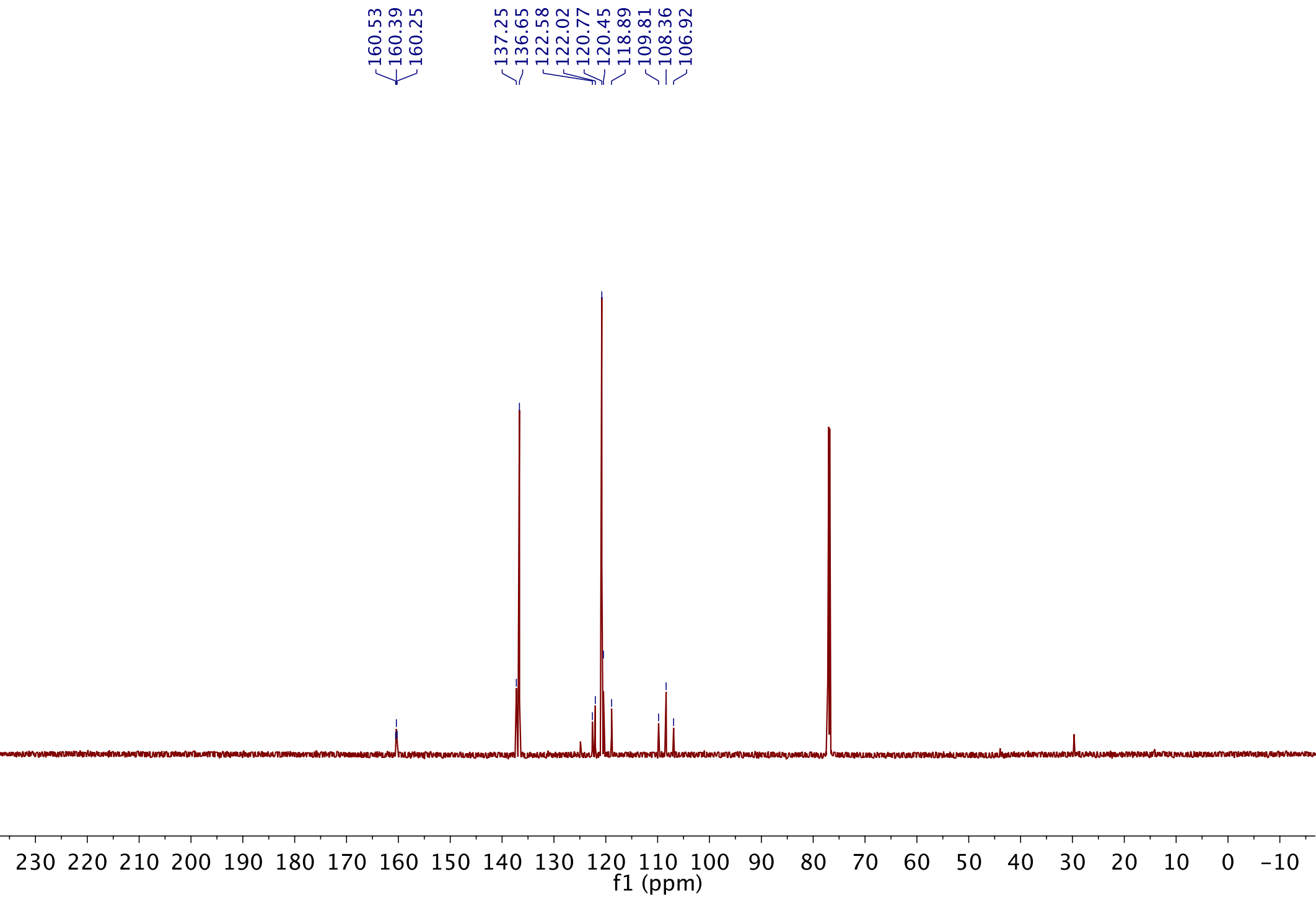
<smiles>O=C(Nc1ccc(SC(F)F)cc1)C(F)(F)F</smiles>

$2 \mathbf{i}$

${ }^{19} \mathrm{~F}$ NMR

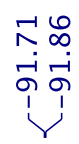

กิ้

น่

V

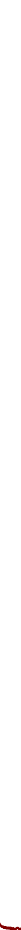

$20 \quad \begin{array}{llllllll} & -80 & -100 & -120 & -140 & -160 & -180 & -200\end{array}$




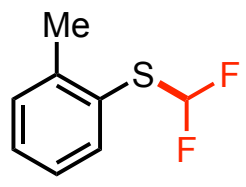

$2 \mathrm{~m}$

${ }^{1} \mathrm{H}$ NMR

더요ำ

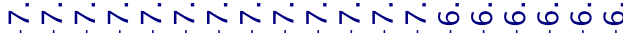

9

f1 $\stackrel{6}{\text { ppm })}$

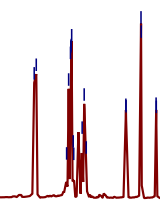

ln 
<smiles>Cc1ccccc1SC(F)F</smiles>

$2 \mathrm{~m}$

${ }^{13} \mathrm{C}$ NMR
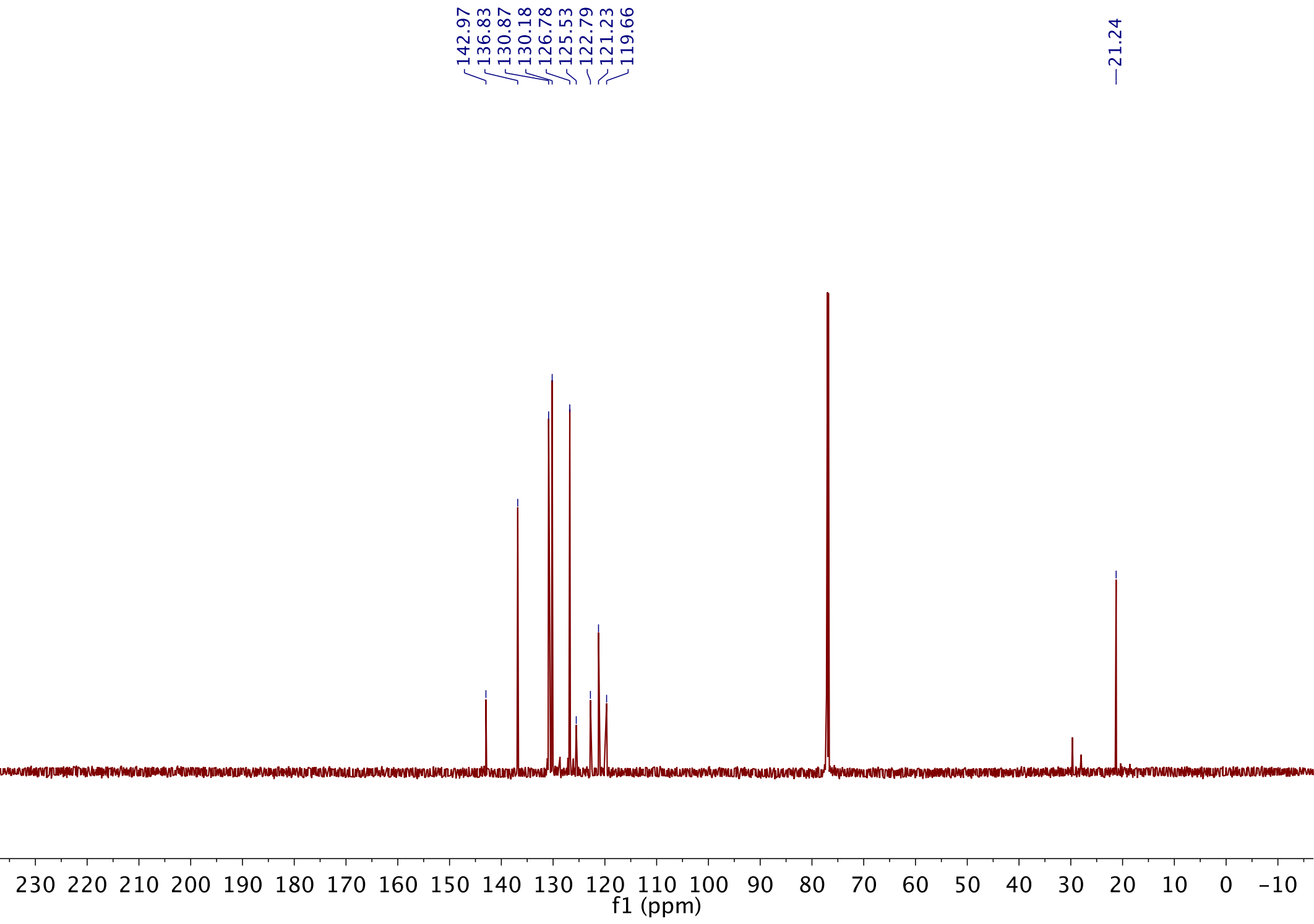


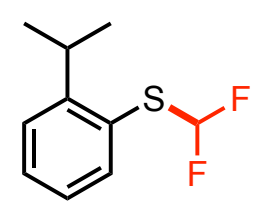

$1 \mathrm{H}$ NMR

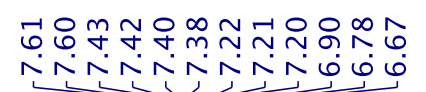

주유유

$m m m m$

$\stackrel{\text { กิ }}{\sim}$

$\wedge \wedge \wedge \wedge \wedge \wedge \wedge 666$
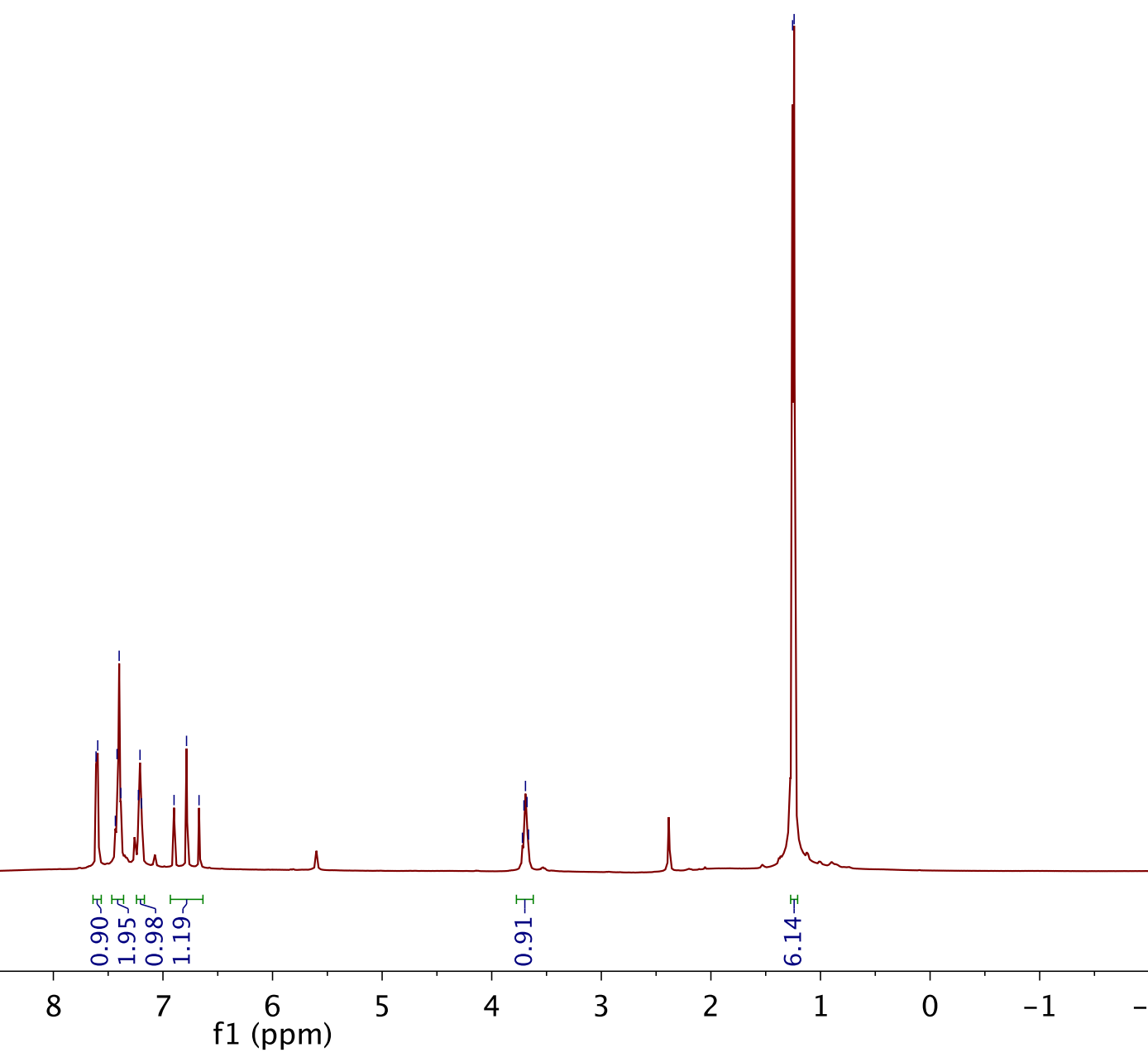


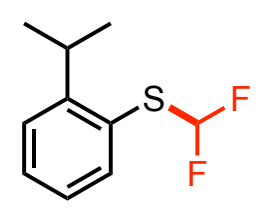

2n

${ }^{13} \mathrm{C}$ NMR
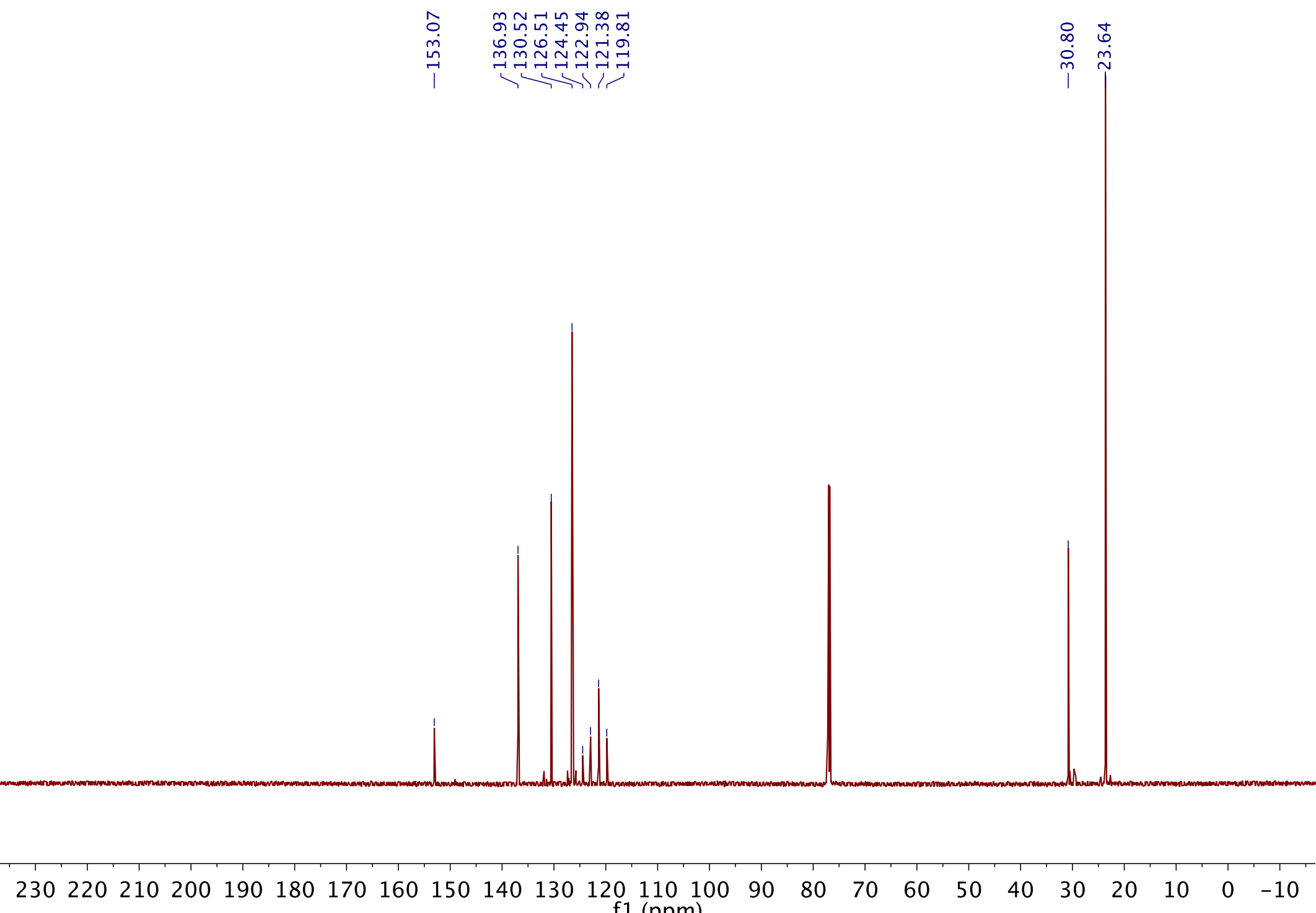

f1 (ppm) 


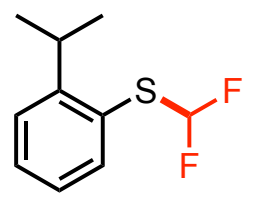

2n

${ }^{19} \mathrm{~F}$ NMR

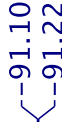
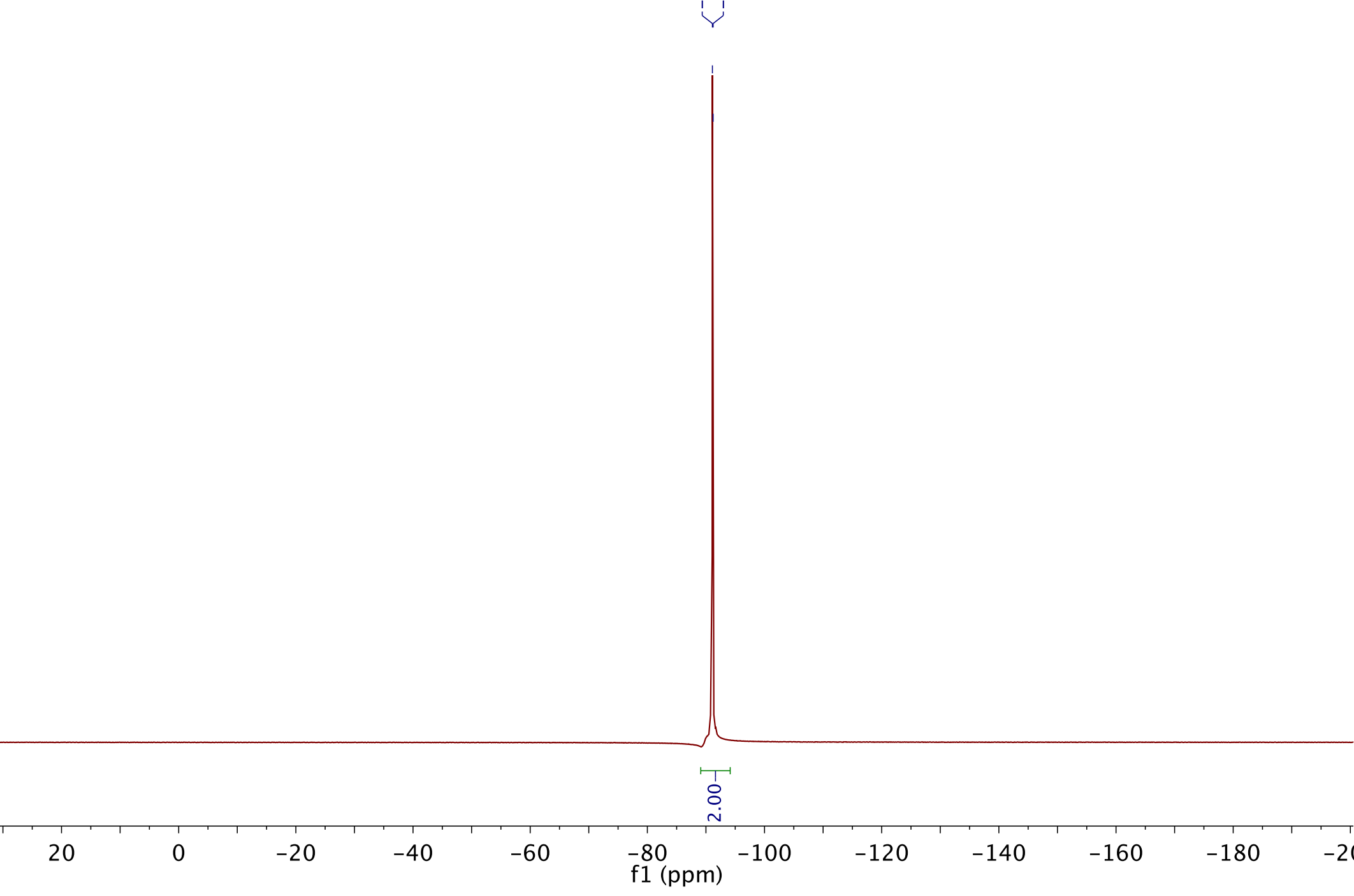


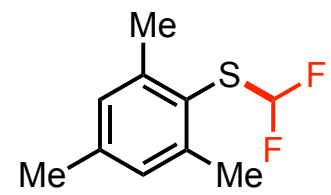

20

${ }^{1} \mathrm{H}$ NMR

도요순

مَ

กิำ

र

in

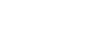


<smiles>Cc1cc(C)c(SC(F)F)c(C)c1</smiles>

20

${ }^{13} \mathrm{C}$ NMR

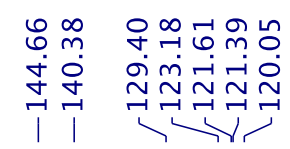

ㄲํㅇ

$\stackrel{\sim}{\sim}$

I

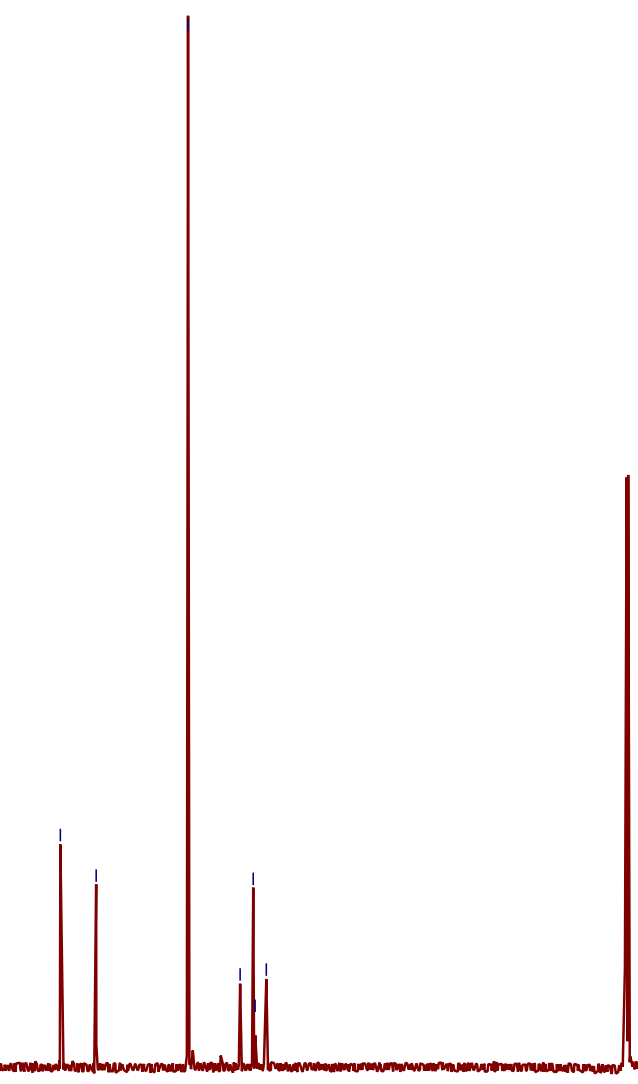

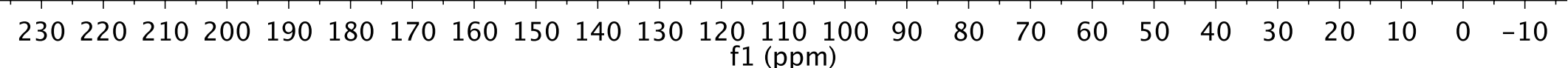




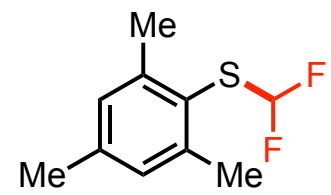

20

${ }^{19} \mathrm{~F}$ NMR

놋

ํํํ

1

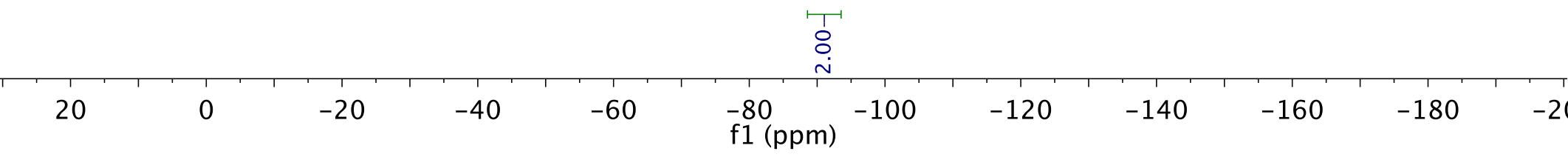


<smiles>FC(F)SCCc1ccccc1</smiles>

$2 p$

${ }^{1} \mathrm{H}$ NMR

以

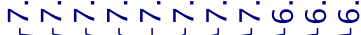

ins
ㅇํㅇํㅇㅇㅛ

mmmm

mmmn

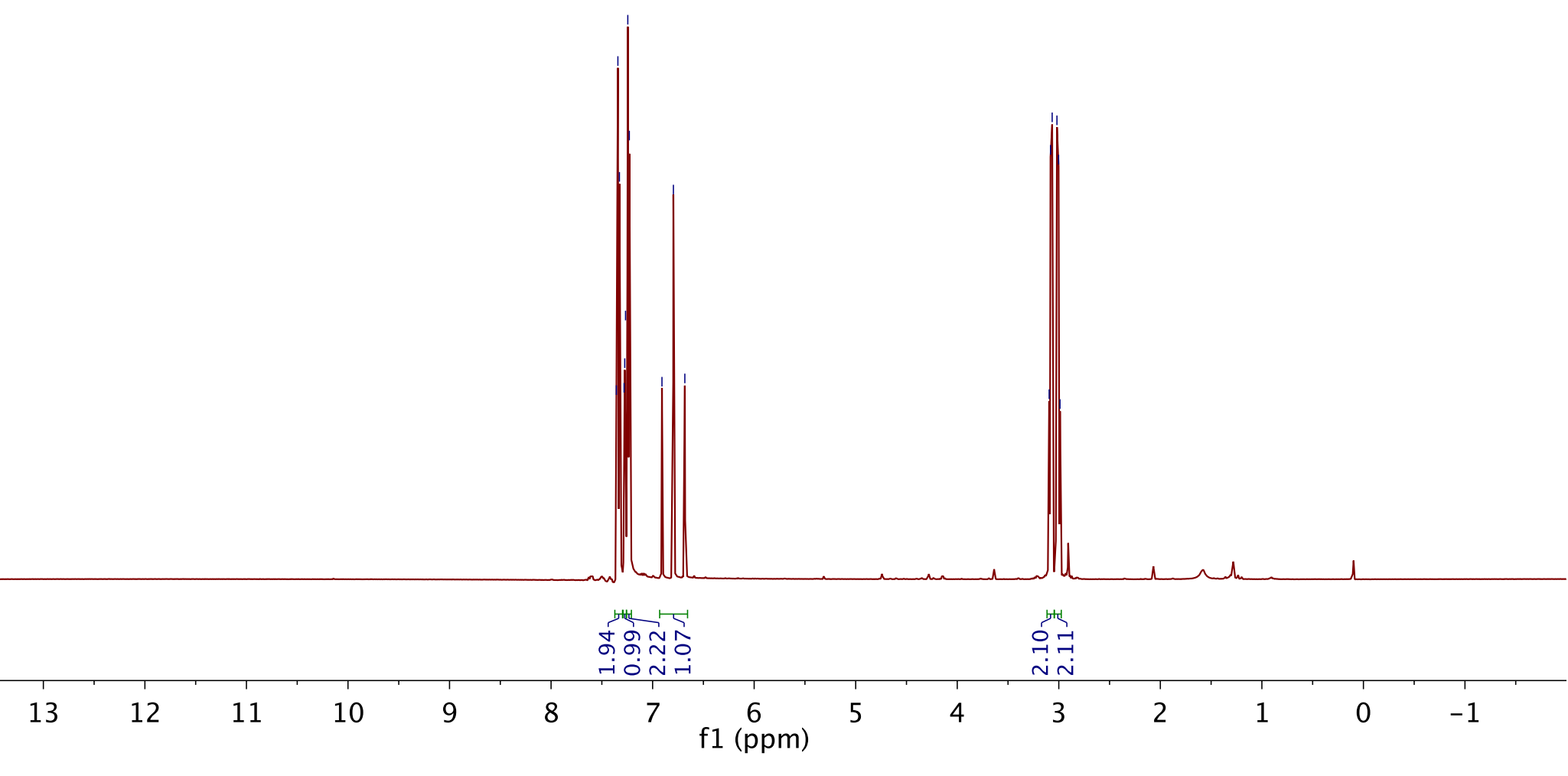


<smiles>FC(F)SCCc1ccccc1</smiles>

$2 p$

${ }^{13} \mathrm{C}$ NMR

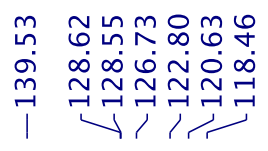

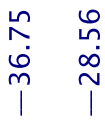

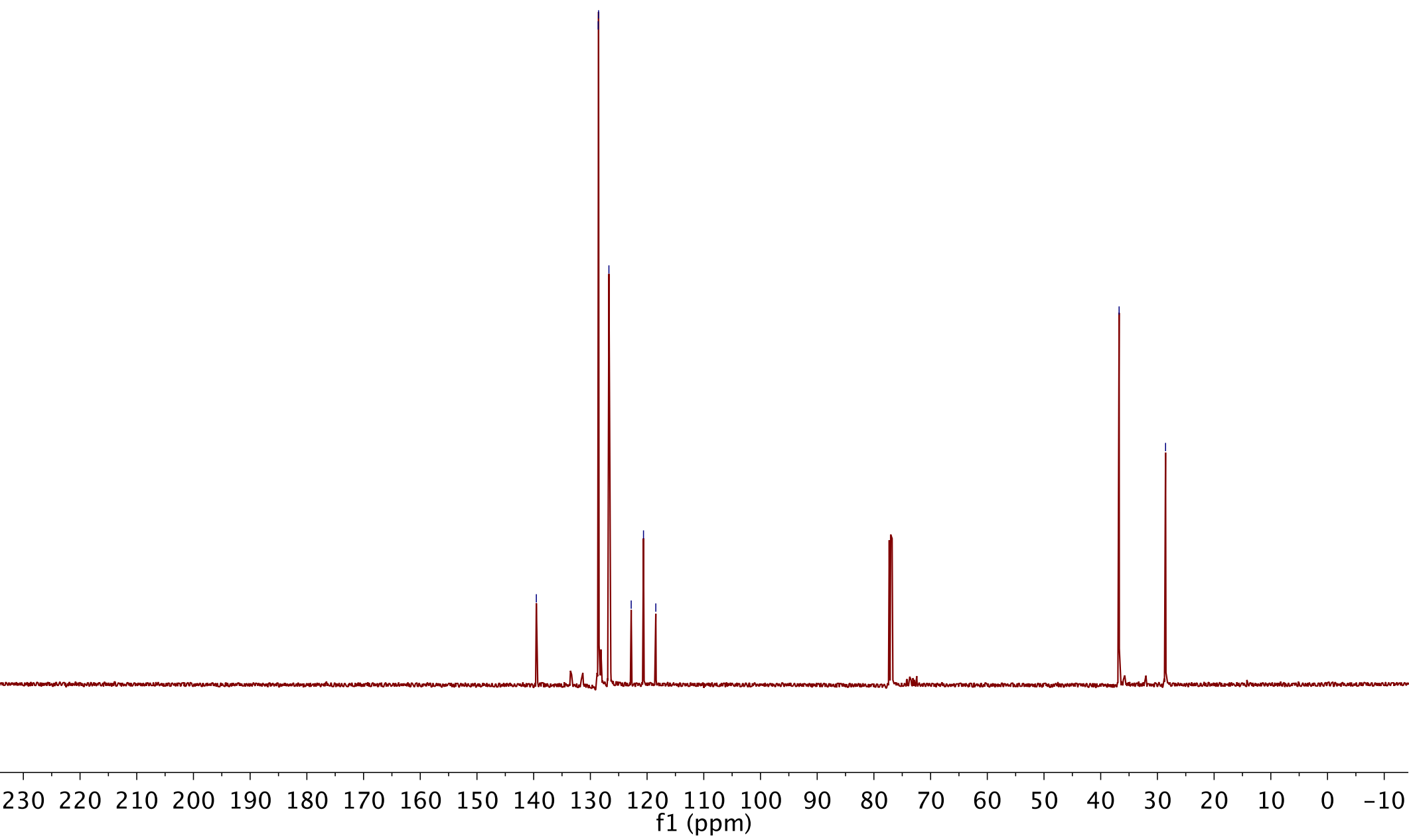


<smiles>FC(F)SCCc1ccccc1</smiles>

$2 p$

${ }^{19} \mathrm{~F}$ NMR

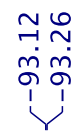

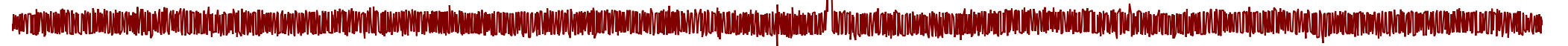

\begin{tabular}{|c|c|c|c|c|c|c|c|c|c|c|c|}
\hline & & & & & & $\begin{array}{l}\text { 'T' } \\
\text { ó } \\
\dot{\sim} \\
\end{array}$ & & & & & \\
\hline 20 & 0 & -20 & -40 & -60 & $\begin{array}{l}-80 \\
\mathrm{f1}(\mathrm{ppm})\end{array}$ & -100 & -120 & -140 & -160 & -180 & $-20 c$ \\
\hline
\end{tabular}




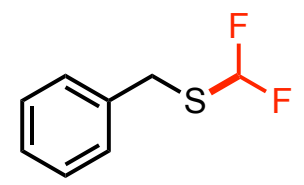

$2 q$

${ }^{1} \mathrm{H}$ NMR

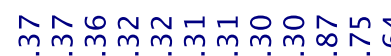

NNNNNNNN6.606

\&

"
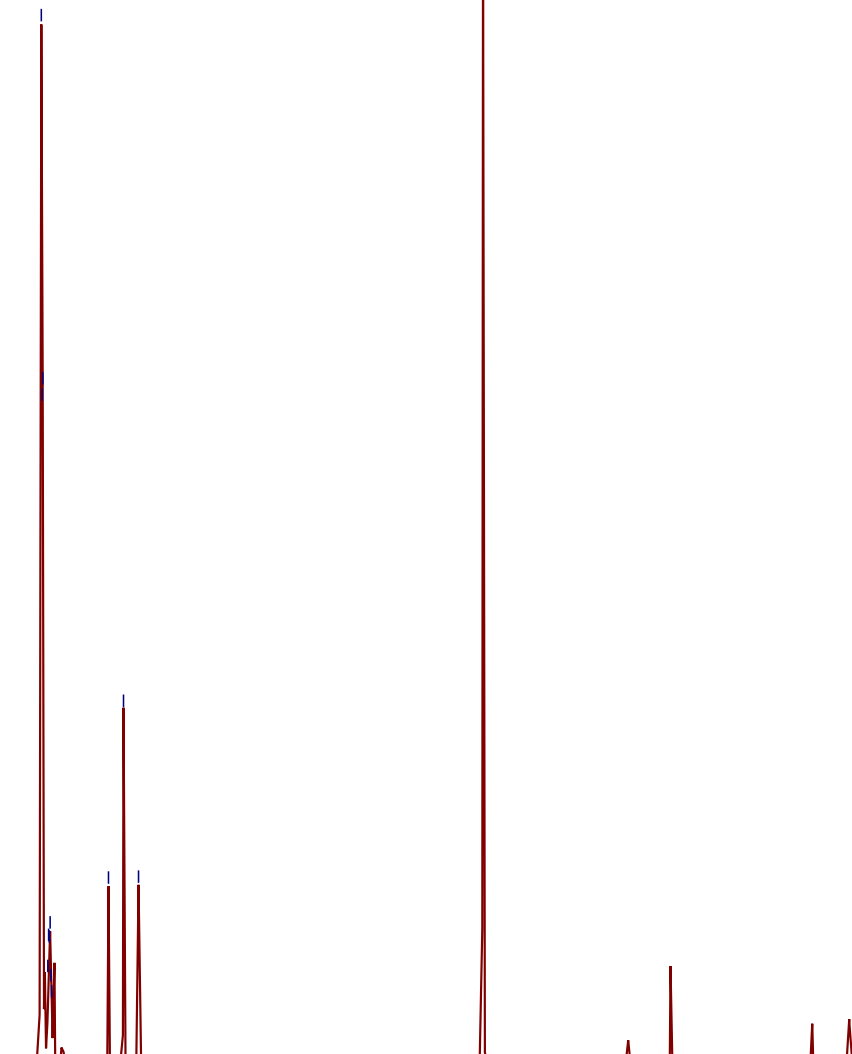

帅 !

मंनं म

$\begin{array}{llllll}13 & 12 & 11 & 10 & 9 & 8\end{array}$

8

7

f1 (ppm)

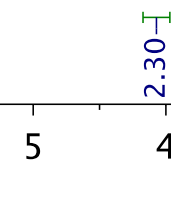


<smiles>FC(F)SCc1ccccc1</smiles>

$2 q$

${ }^{13} \mathrm{C}$ NMR

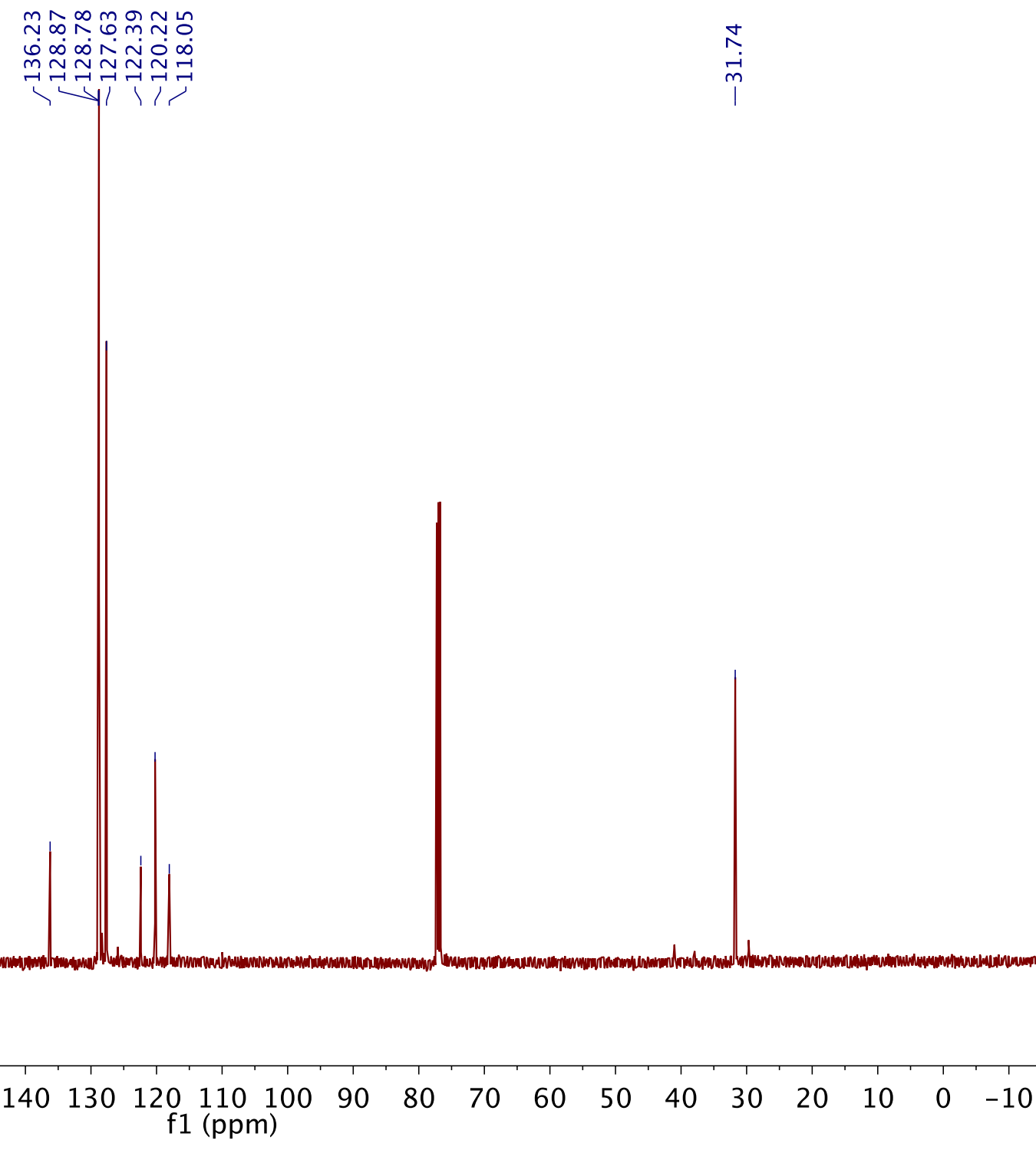




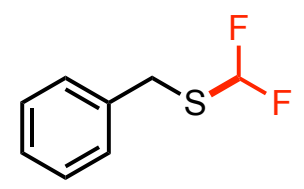

$2 q$

${ }^{19} \mathrm{~F}$ NMR

용

ป்

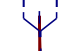

m.r.

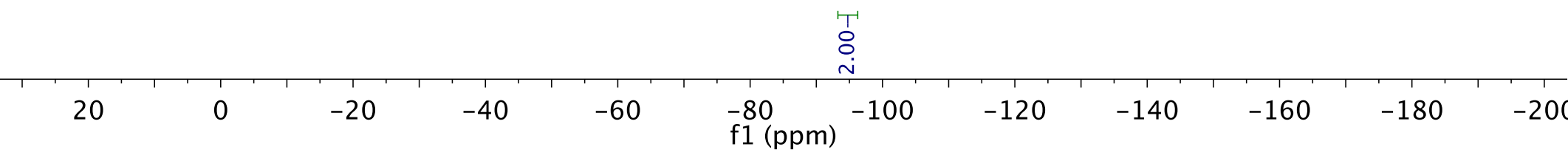




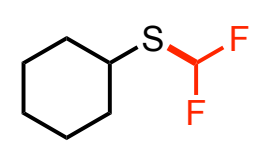

2s

${ }^{1} \mathrm{H}$ NMR

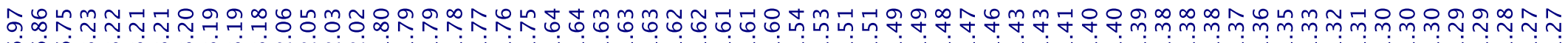
ம

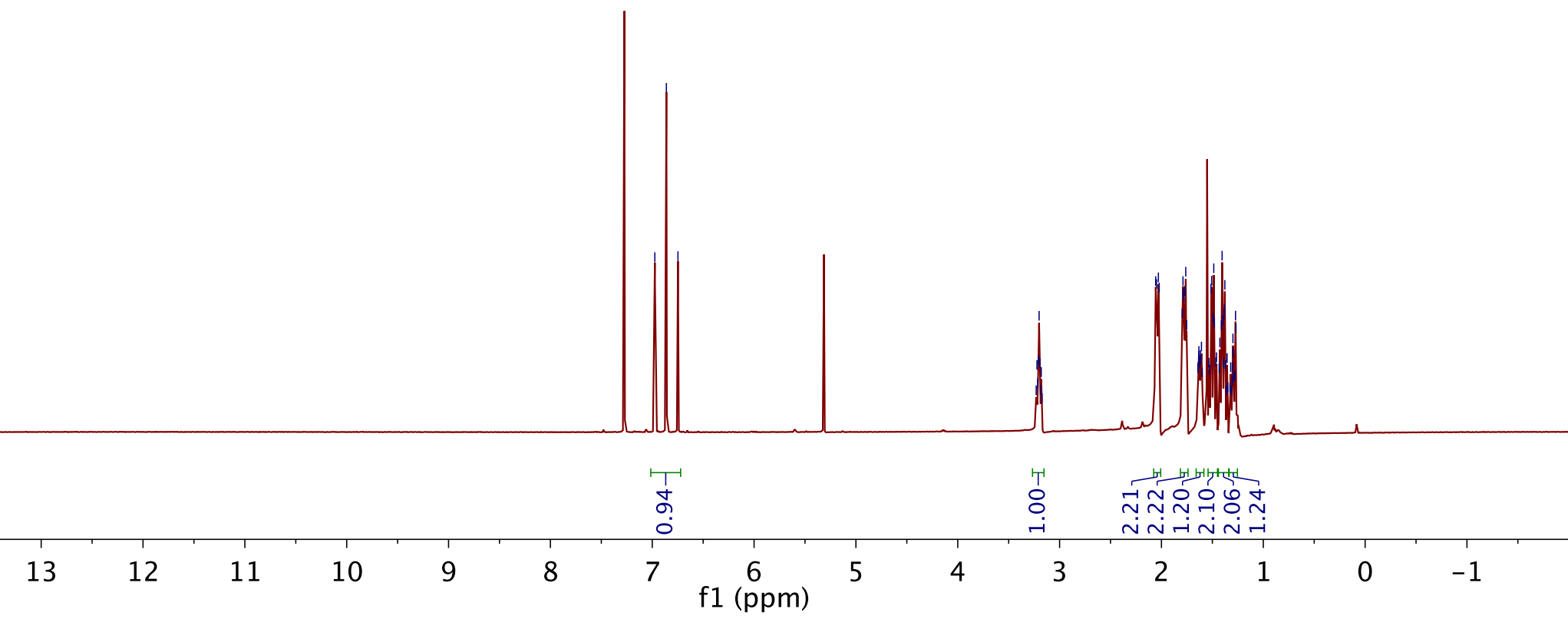


<smiles>FC(F)SC1CCCCC1</smiles>

2s

${ }^{13} \mathrm{C}$ NMR

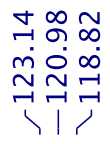
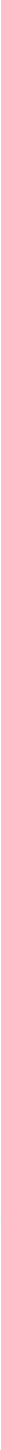
f1 (ppm) 


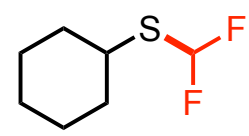

2s

${ }^{19} \mathrm{~F}$ NMR

우눈

नंनुन

I'

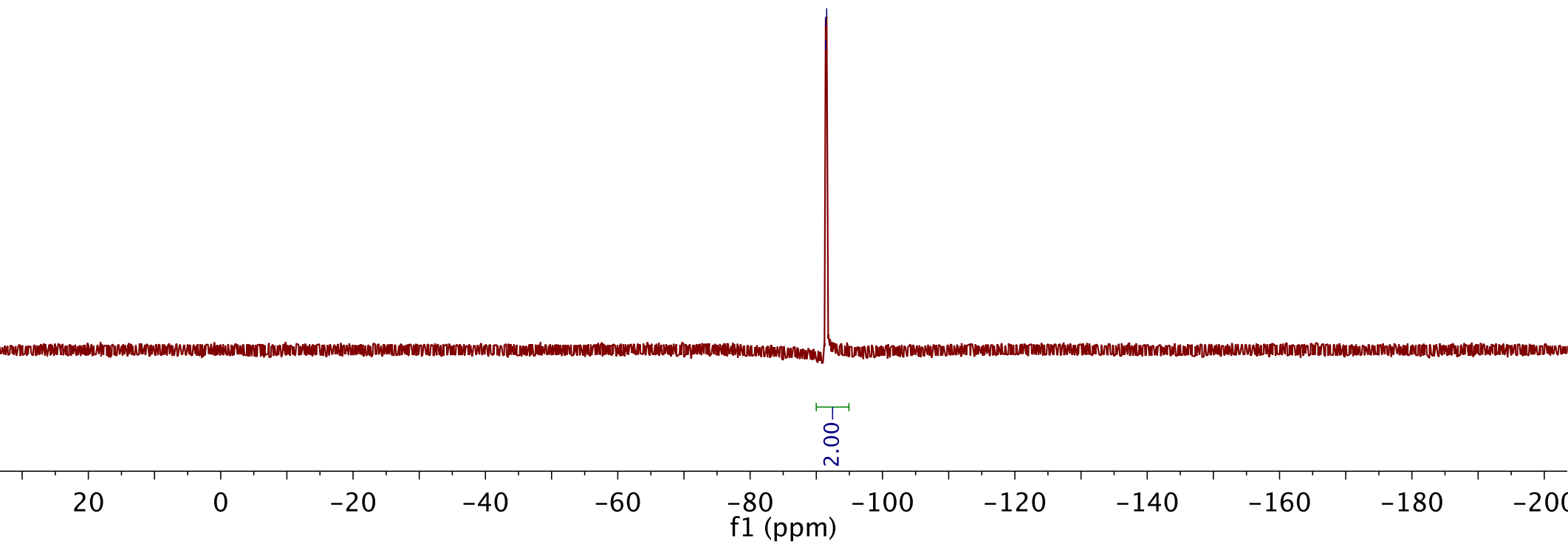




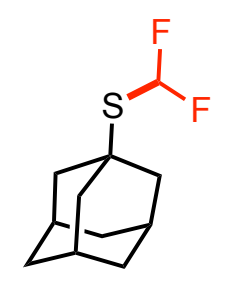

$2 t$

${ }^{1} \mathrm{H}$ NMR

응요

ヘंம்

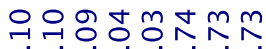

viñinitivin

\I
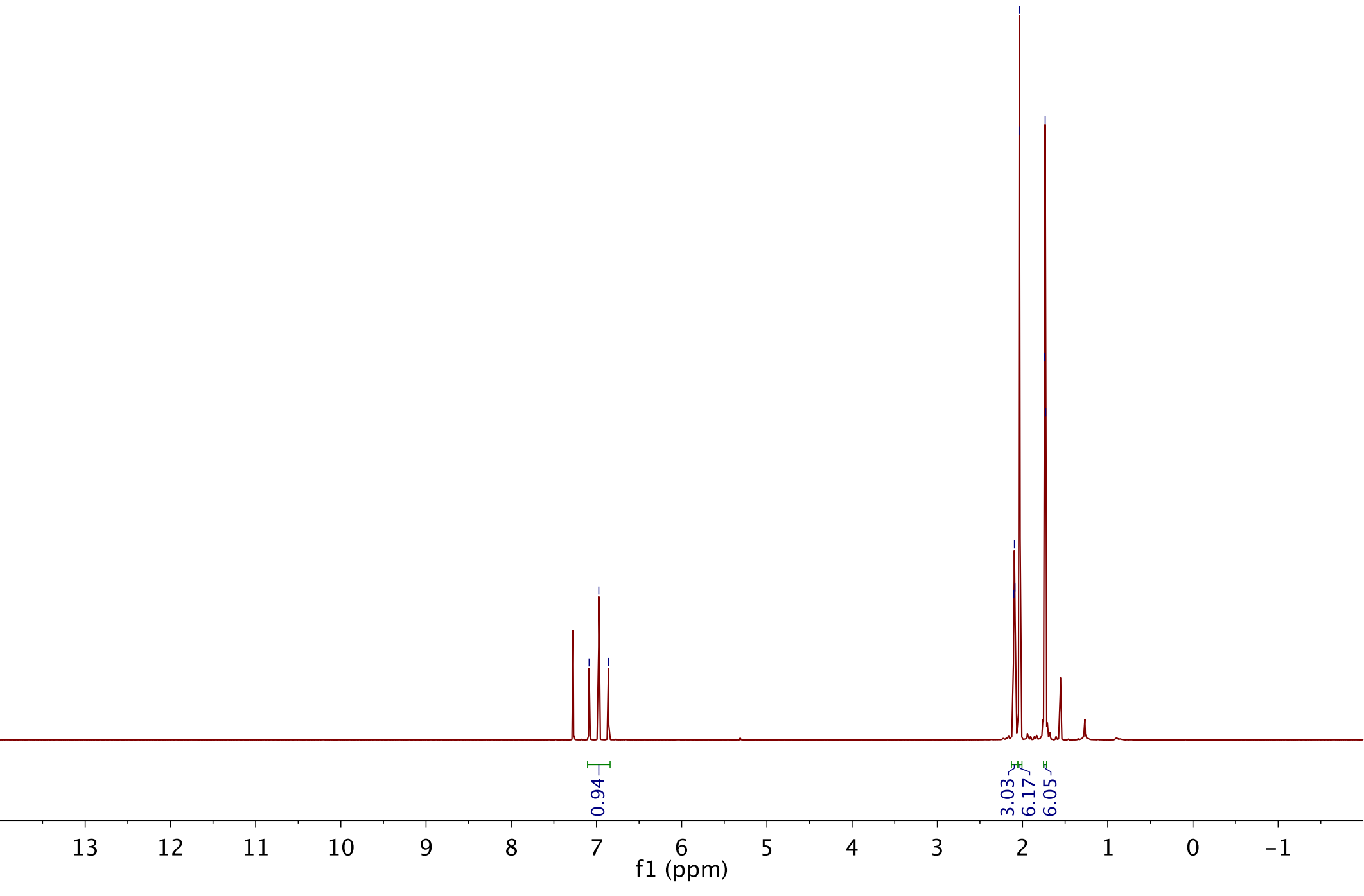


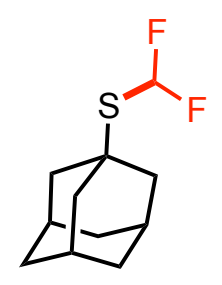

2t

${ }^{19} \mathrm{~F}$ NMR

우소

कें

Y

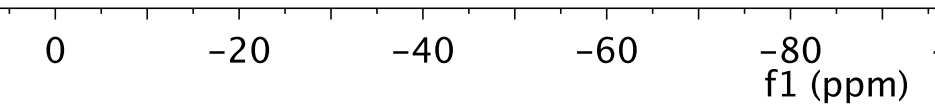

$-100$

\begin{tabular}{lllll|l|}
-120 & -140 & -160 & -180 & -2
\end{tabular}


<smiles>COC(C)CCOC(=O)CCSC(F)F</smiles>

$2 \mathrm{u}$

${ }^{1} \mathrm{H}$ NMR

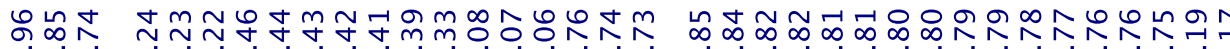

மம

11 il
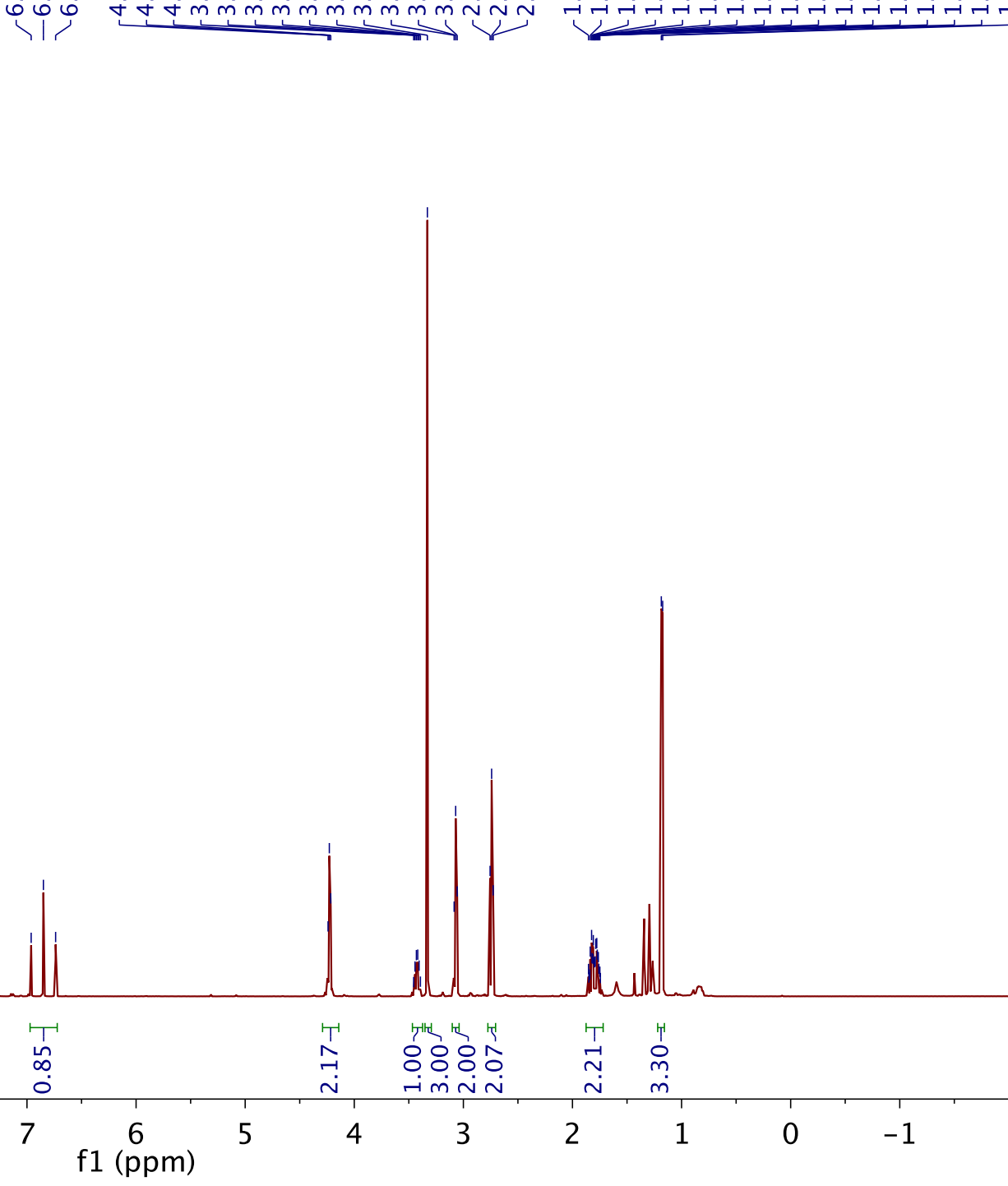
<smiles>COC(C)CCOC(=O)CCSC(F)F</smiles>

$2 u$

${ }^{19} \mathrm{~F}$ NMR

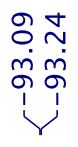

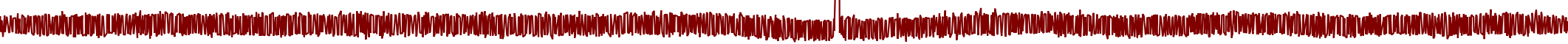

\begin{tabular}{|c|c|c|c|c|c|c|c|c|c|c|c|}
\hline & & & & & $\begin{array}{l}1 \\
8 \\
8\end{array}$ & & & & & & \\
\hline 20 & 0 & -20 & -40 & -60 & $\begin{array}{l}-80 \\
\mathrm{f1}(\mathrm{ppm})\end{array}$ & -100 & -120 & -140 & -160 & -180 & -200 \\
\hline
\end{tabular}




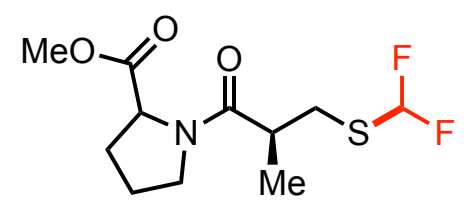

2v

${ }^{1} \mathrm{H}$ NMR

น ט
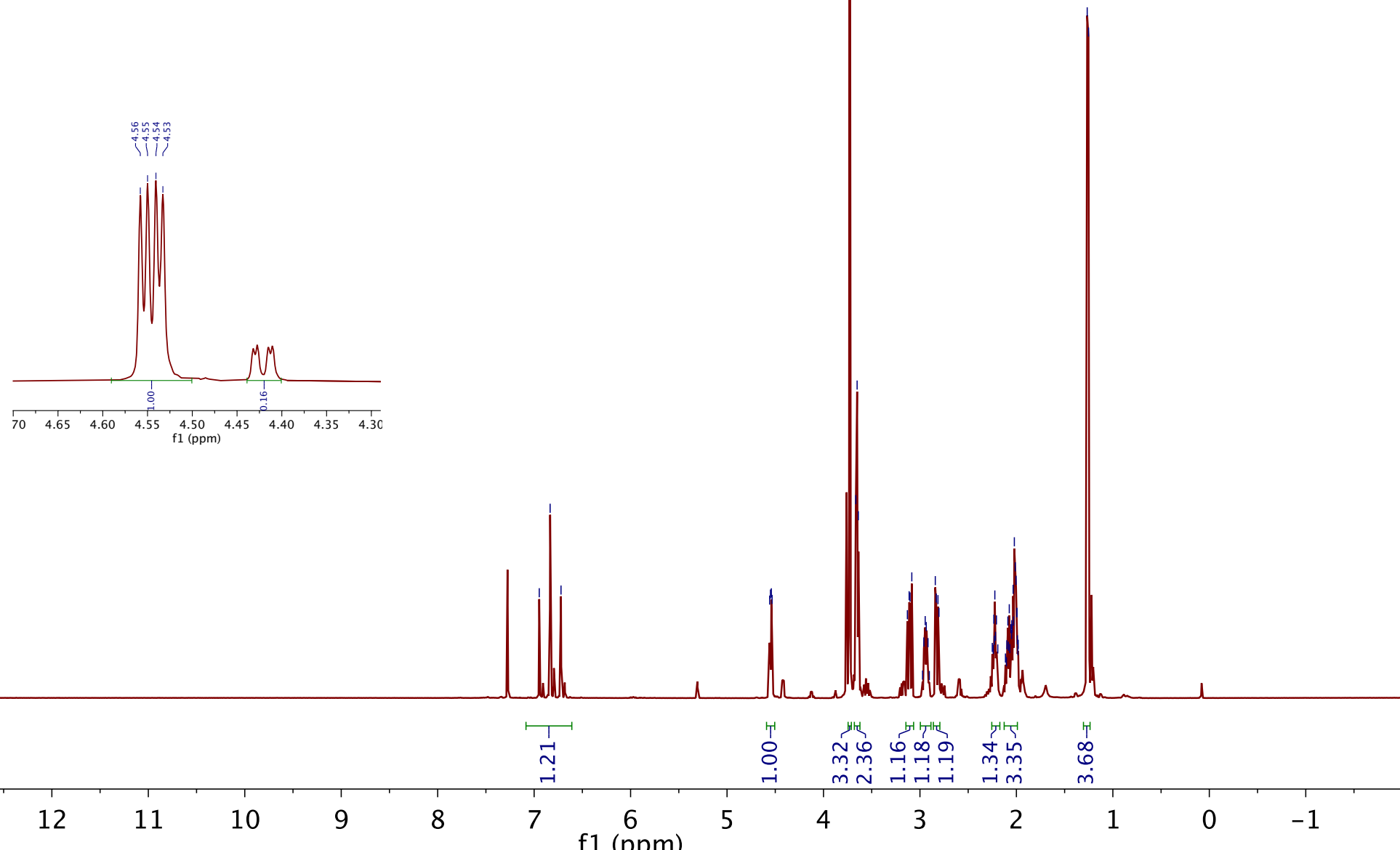


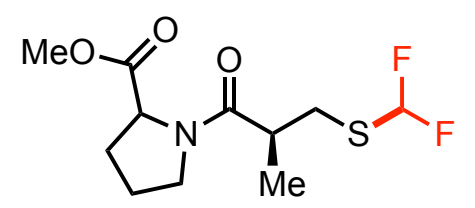

2v

${ }^{13} \mathrm{C}$ NMR

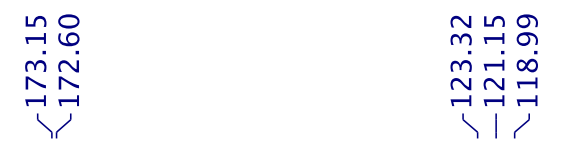

느응ㅇㅇㅇ

겅ํㅜ응

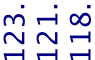

นกำ

엄스

I ।

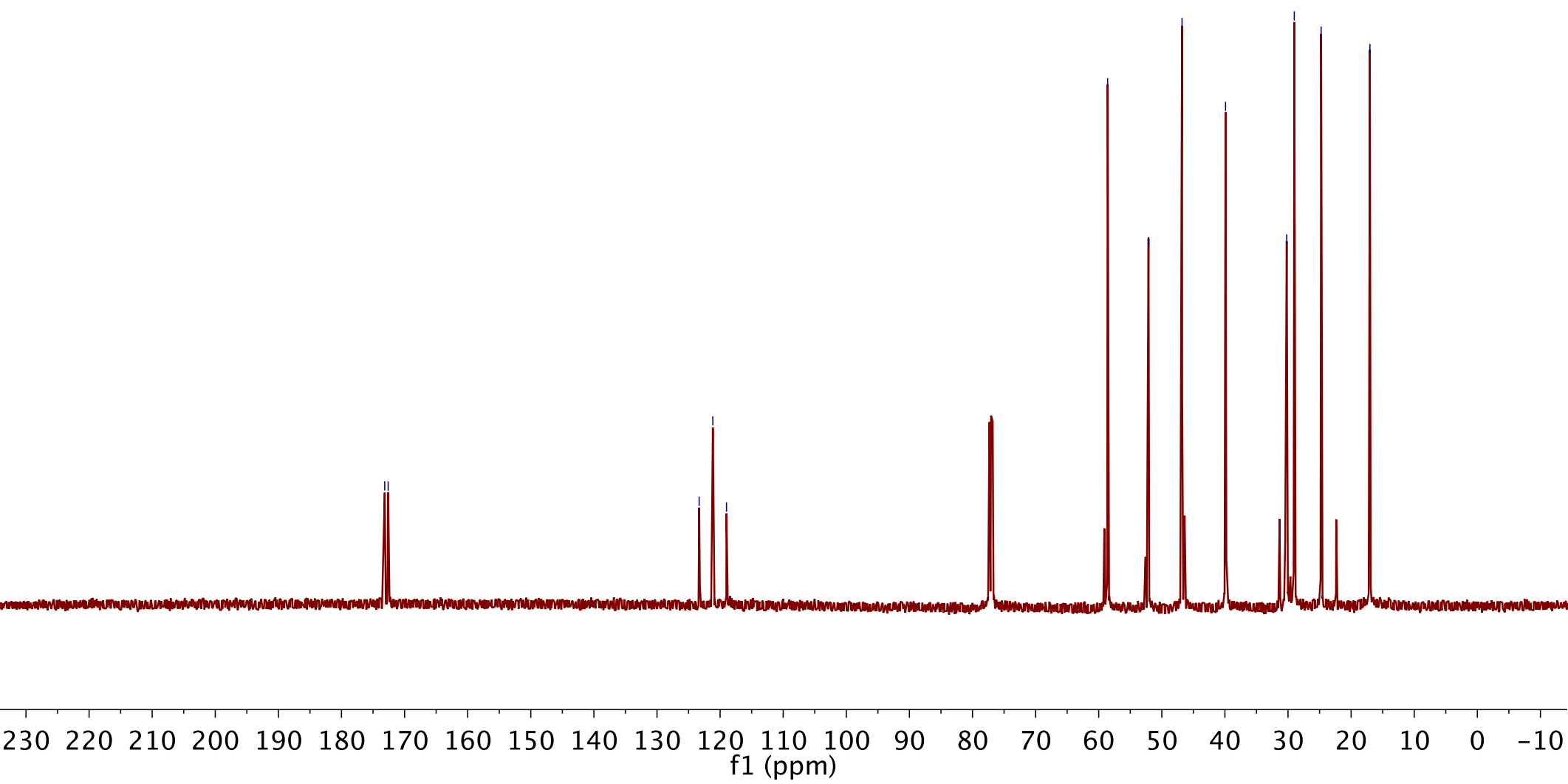




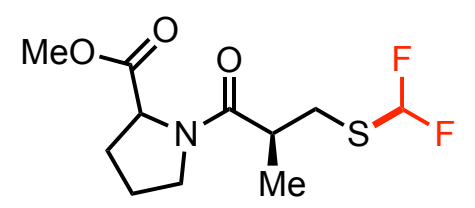

2v

${ }^{19}$ F NMR

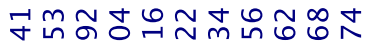

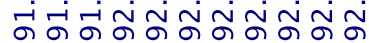

कीष

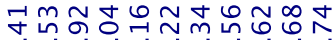

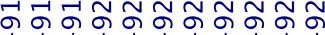

1

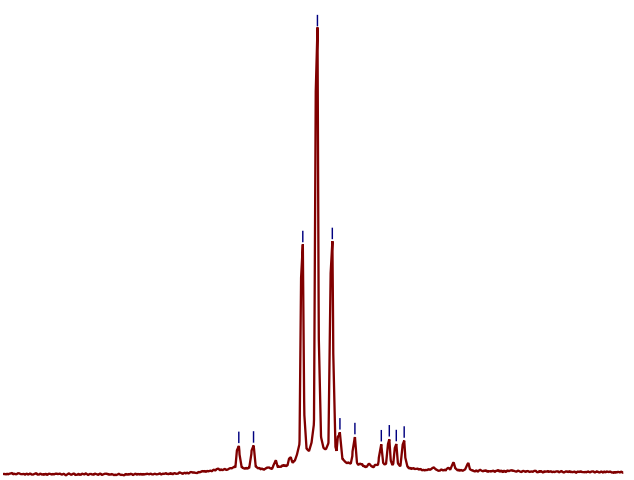

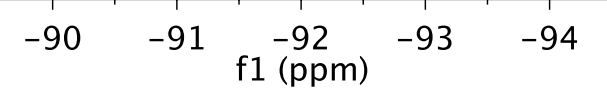

$0+-2$

$-20$

$-40$

$-60$

$-80$

$-100$

$-120$

$-140$

$-160$

$-180$

$-2$

f1 (ppm) 


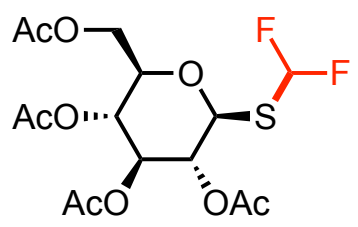

$2 w$

${ }^{1} \mathrm{H} N M R$
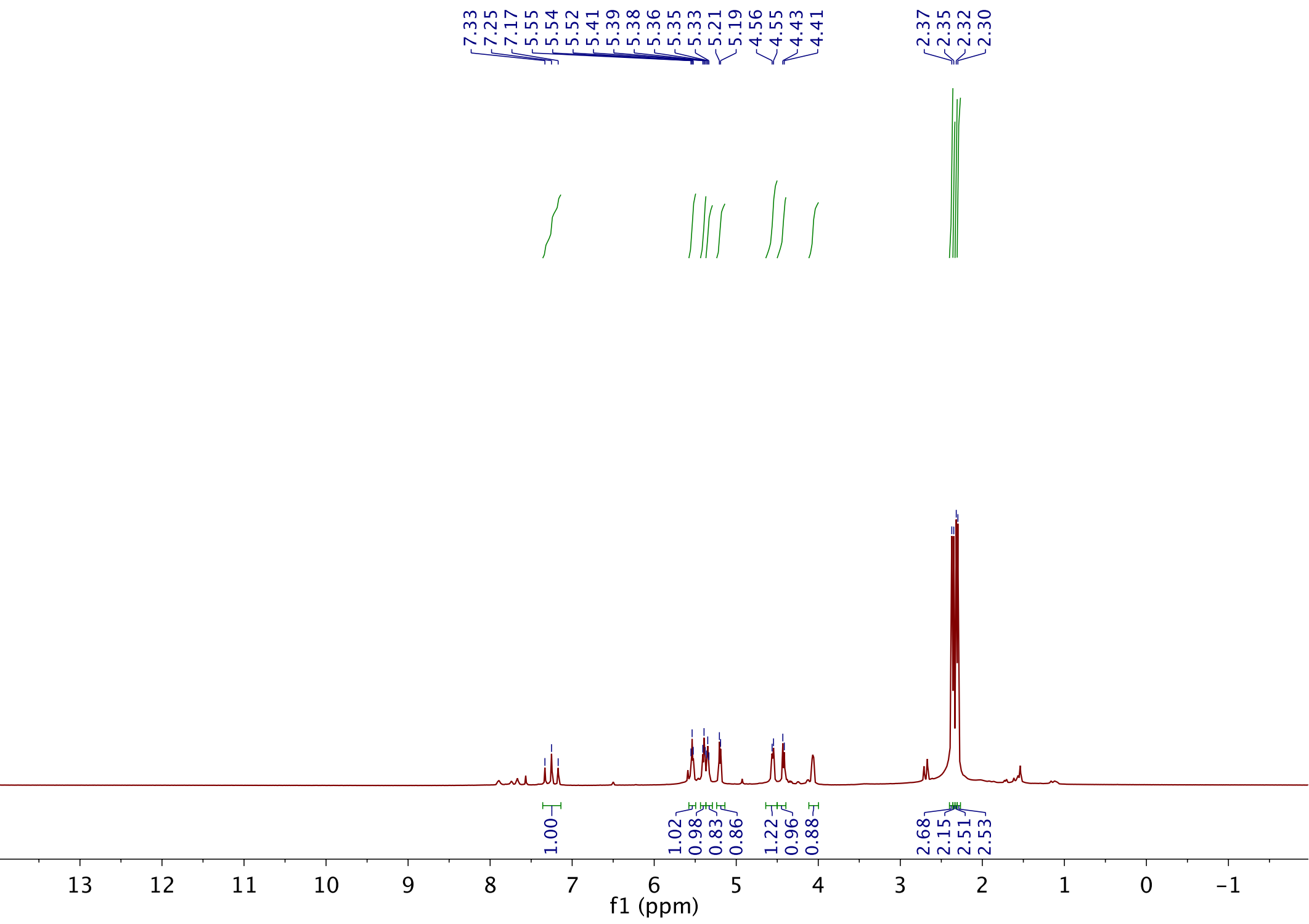


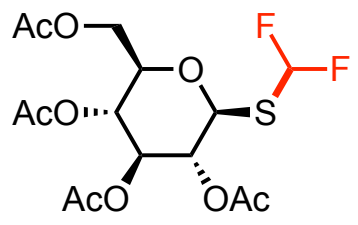

2w

${ }^{13} \mathrm{C}$ NMR

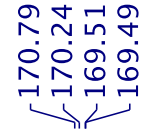

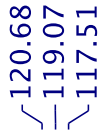

ำำㅇํㅇำ

앗Nㅅㅇㅠ

11। 1 ।

$\stackrel{\infty}{\stackrel{0}{N}}$

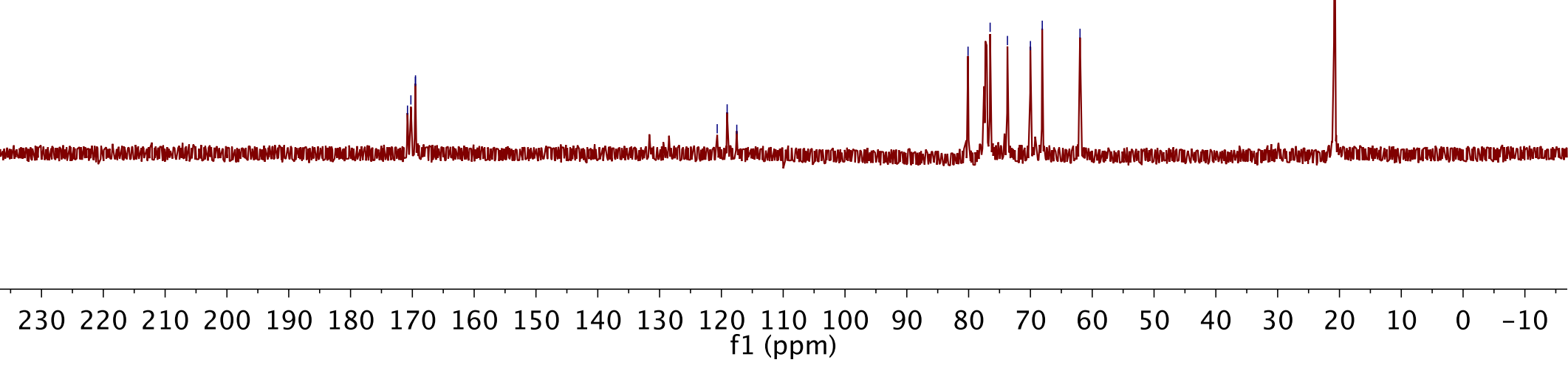




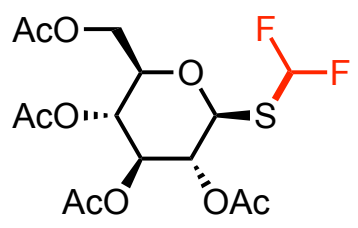

$2 w$

${ }^{19} \mathrm{~F}$ NMR

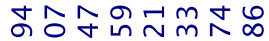

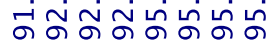

1 का का का

$\longrightarrow$

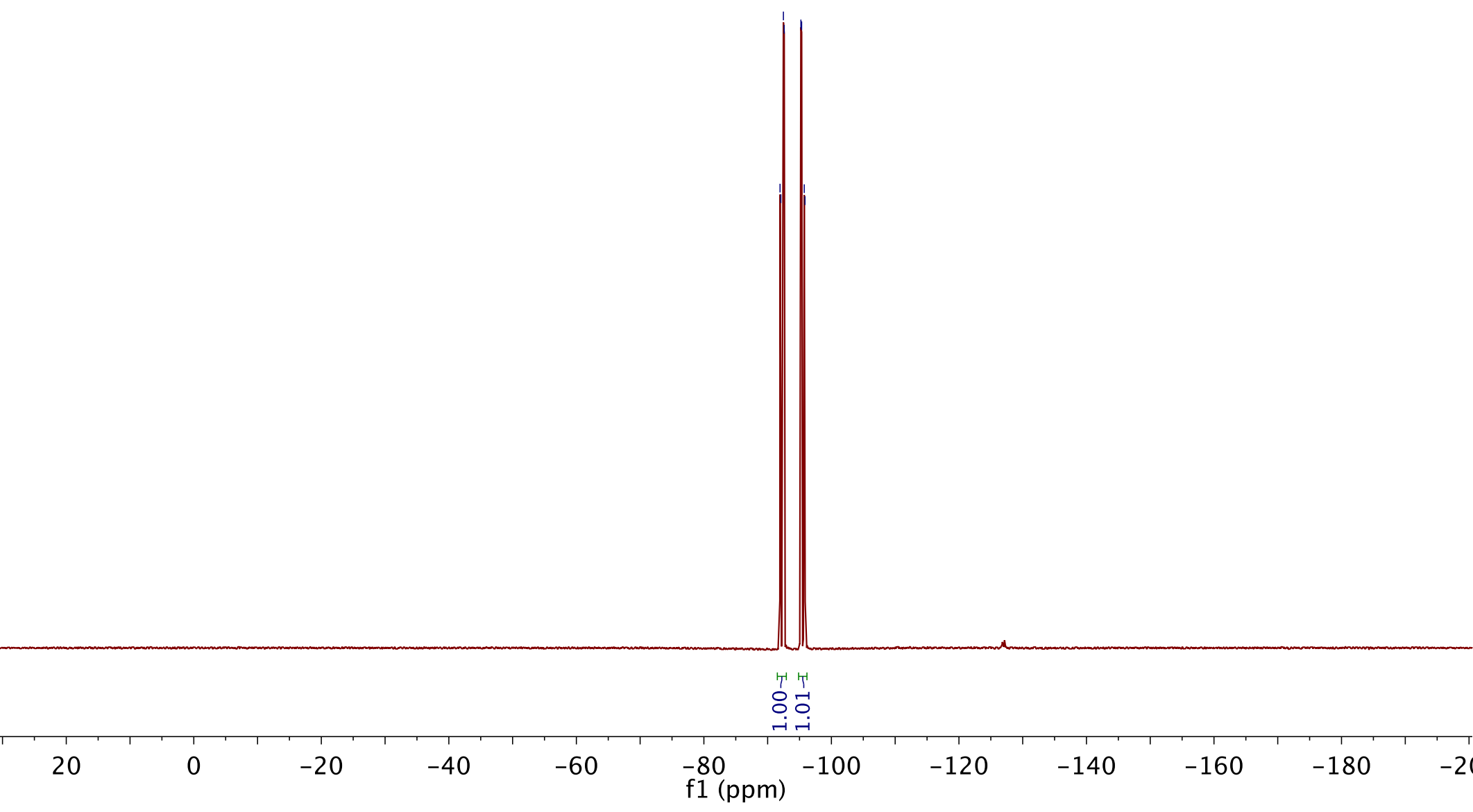


<smiles>COc1ccc(SCF)cc1</smiles>

2x

${ }^{1} \mathrm{H}$ NMR

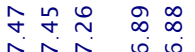

Ni,

ใิ

था

$-\infty$

i

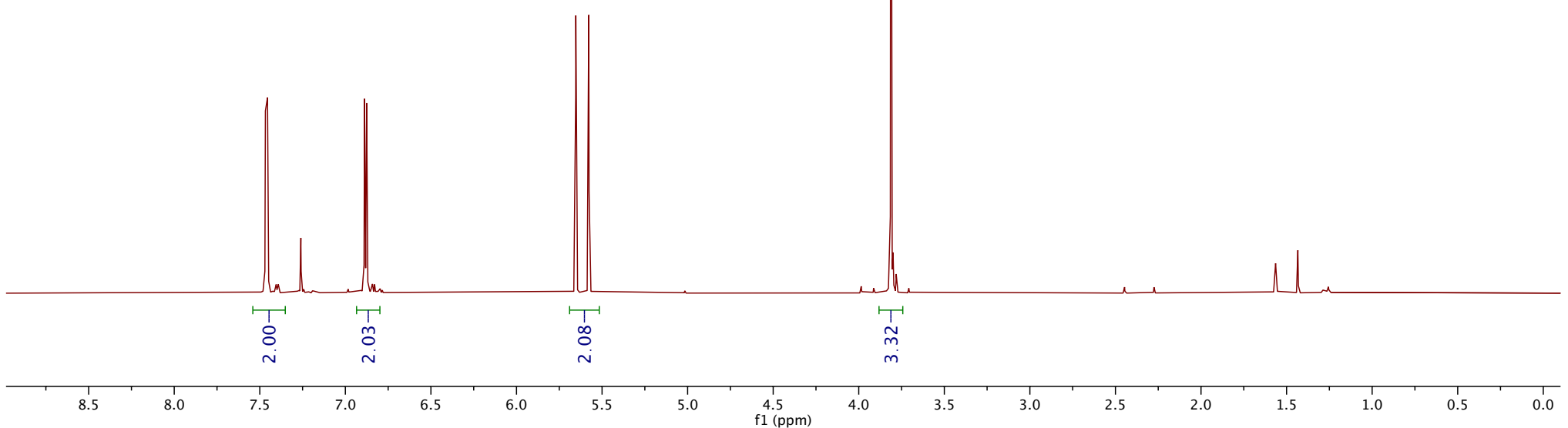




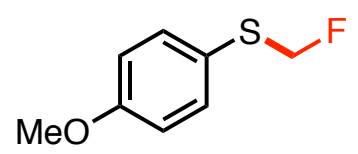

$2 x$

${ }^{13} \mathrm{C}$ NMR

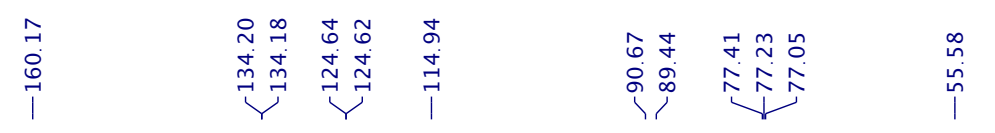
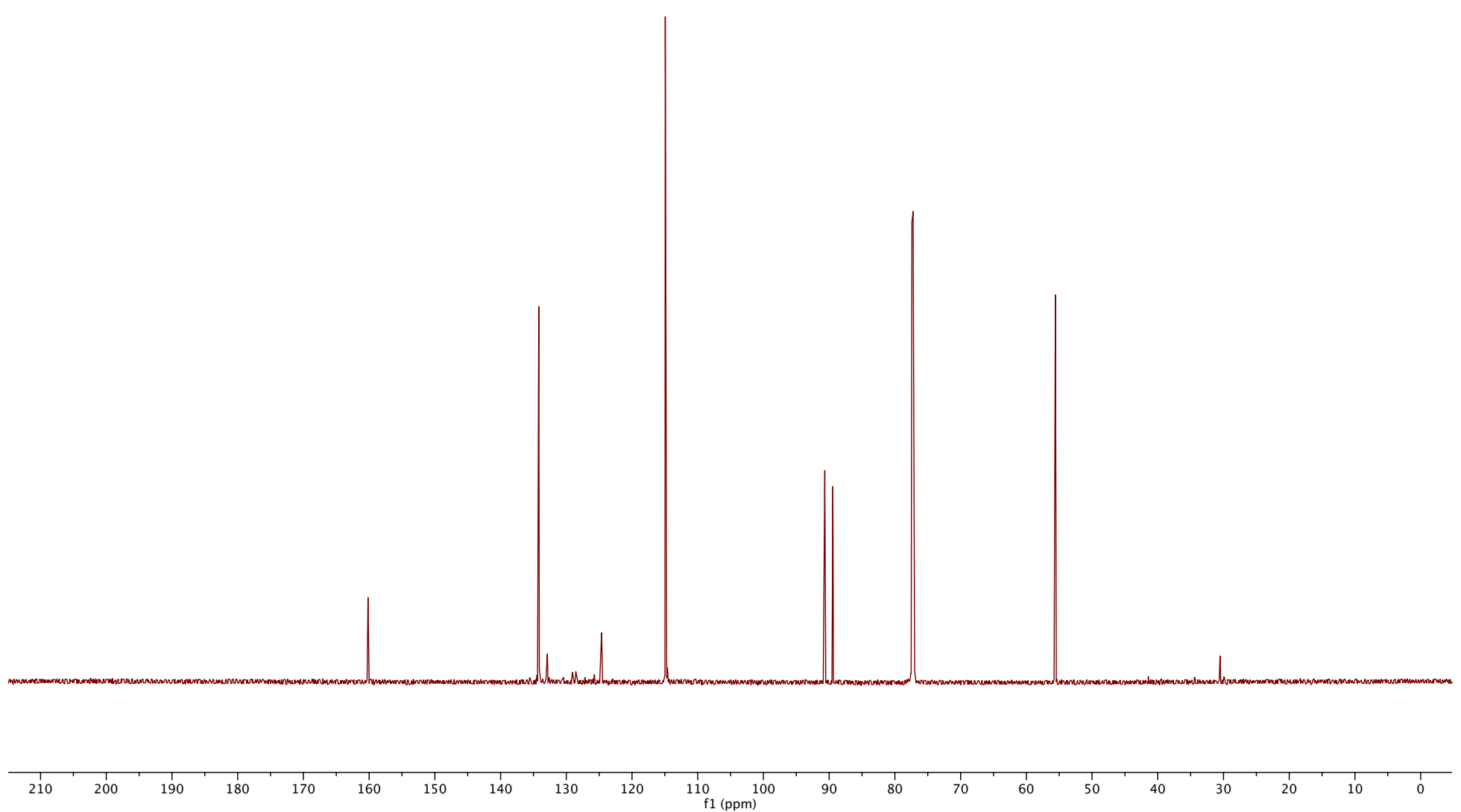
${ }^{S}{ }^{\mathrm{S}}$

2x

${ }^{19} \mathrm{~F}$ NMR

아웅

$\underset{\sim}{\vec{\infty}} \underset{-1}{-\infty}$

$11<$

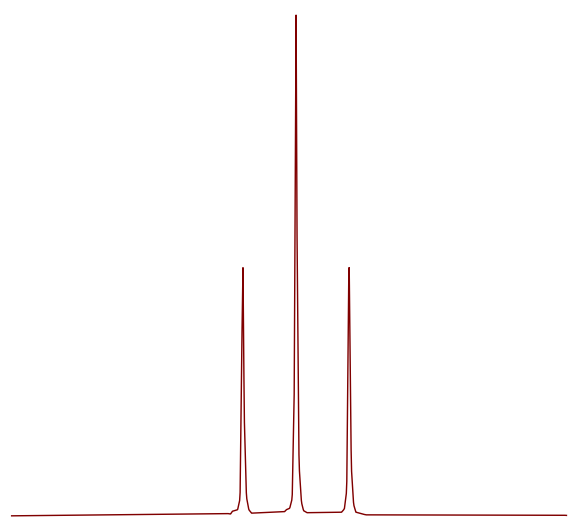

$-181.0$

$-182.0$

-181.5
f1 (ppm)

f1 (ppm)
웅소

$\stackrel{\infty}{\rightarrow} \underset{\sim}{-\infty}$

$\psi$

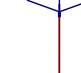

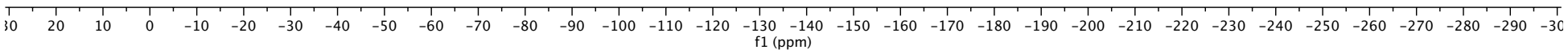




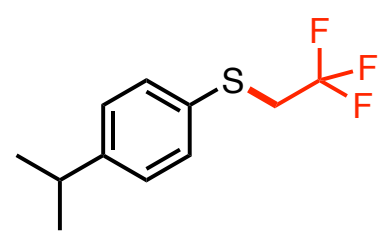

$2 y$

${ }^{1} \mathrm{H}$ NMR

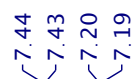

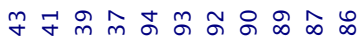

mimm

$\stackrel{\stackrel{n}{\sim}}{\stackrel{+}{*}}$

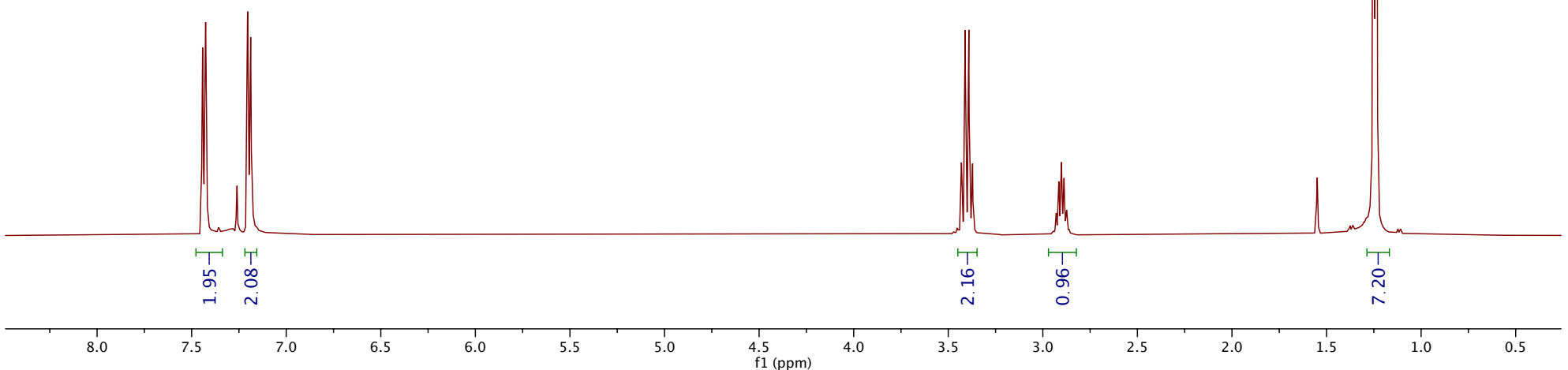


<smiles>CC(C)c1ccc(SCC(F)(F)F)cc1</smiles>

$2 y$

${ }^{13} \mathrm{C}$ NMR

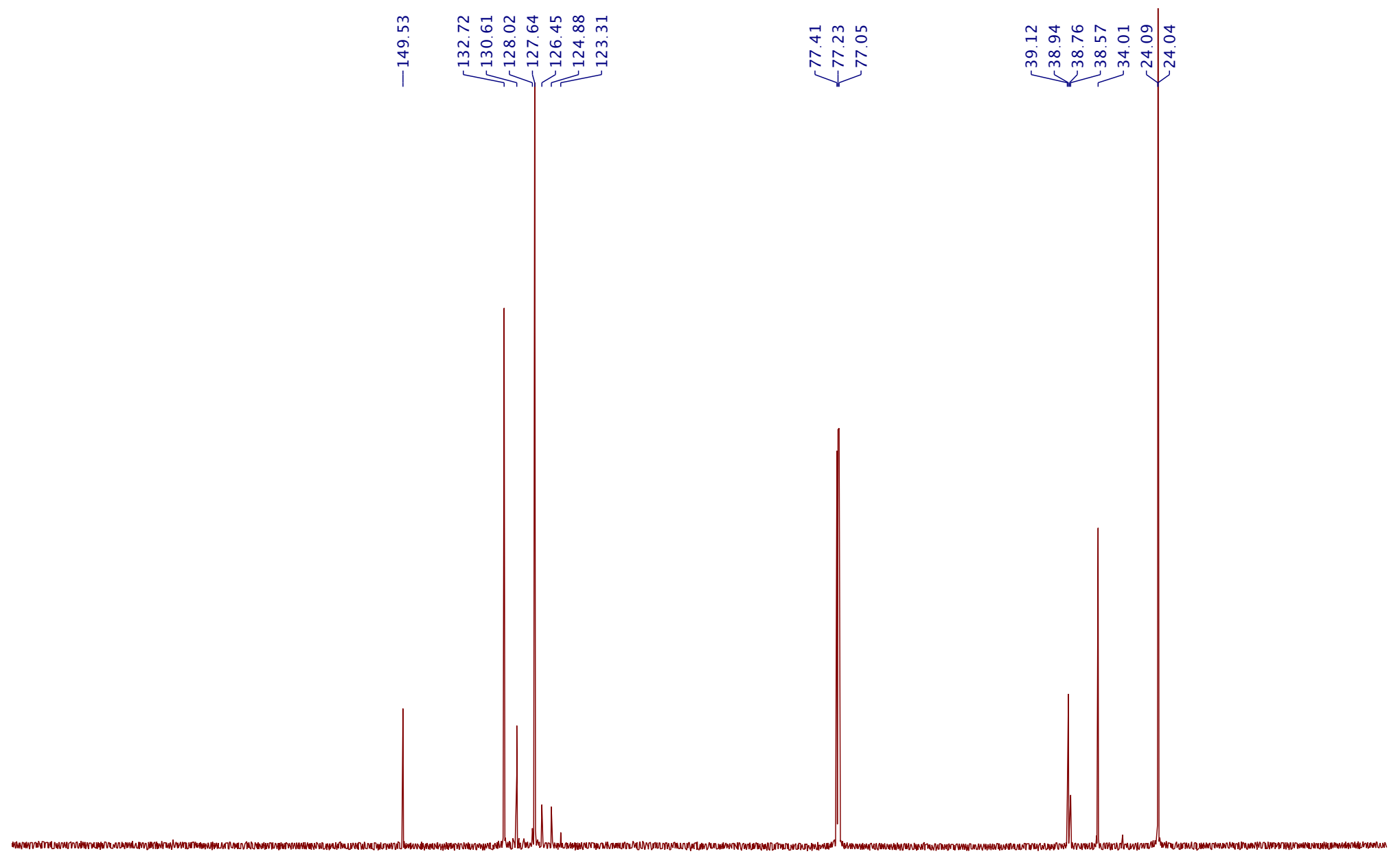

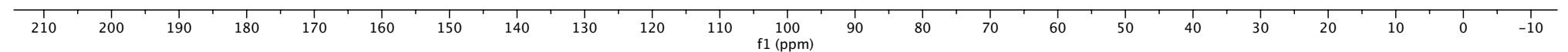




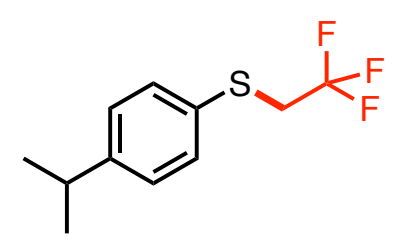

$2 y$

${ }^{19} \mathrm{~F}$ NMR

슬

\& : :

4

50

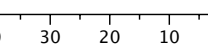

$\begin{array}{lllllllllll}1 & 1 & 1 & 1 & 1 & 1 & 1 & 1 & 1 & 1 & 1 \\ 10 & 0 & -10 & -20 & -30 & -40 & -50 & -60 & -70 & -80\end{array}$

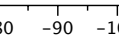
\begin{tabular}{|llllllllllllllllllllllll}
$\mid$ & 100 & -110 & -120 & -130 & -140 & -150 & -160 & -170 & -180 & -190 & -200 & -210 & -220 & -230 & -240 & -250 & -260 & -27
\end{tabular} 


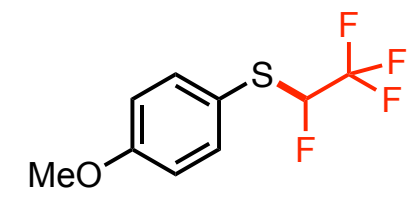

$2 z$

${ }^{1} \mathrm{H}$ NMR

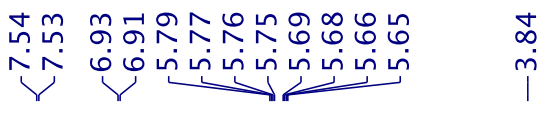

テ

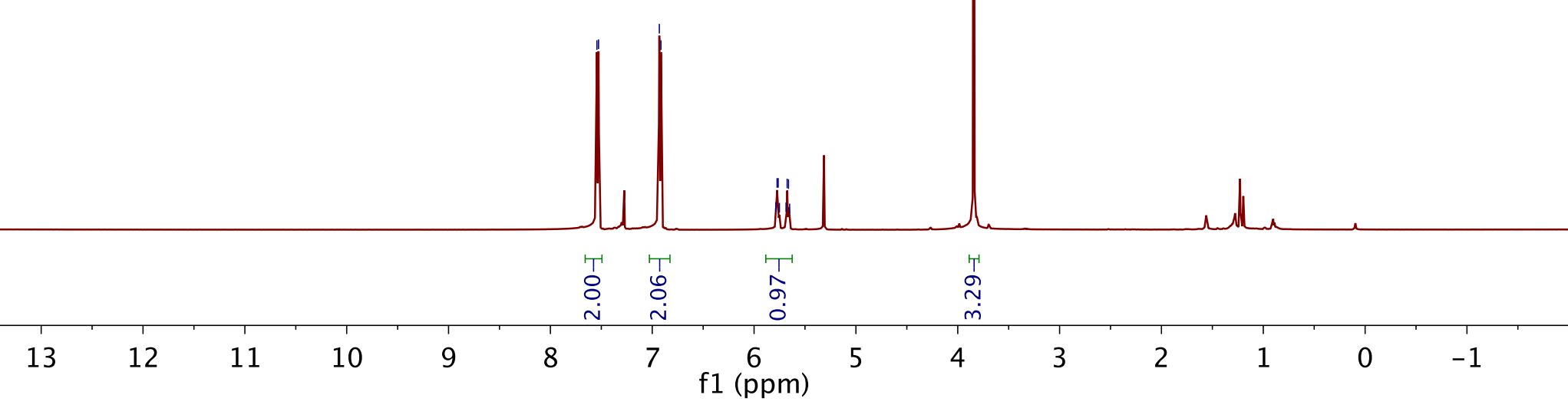


<smiles>COc1ccc(SC(F)C(F)(F)F)cc1</smiles>

$2 z$

${ }^{19} \mathrm{~F}$ NMR

ํํํํำ

ம்

11

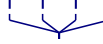

๖ㅇㅠㅎㅀㄴ

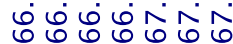

$\begin{array}{lll}1 & 1 & 1\end{array}$

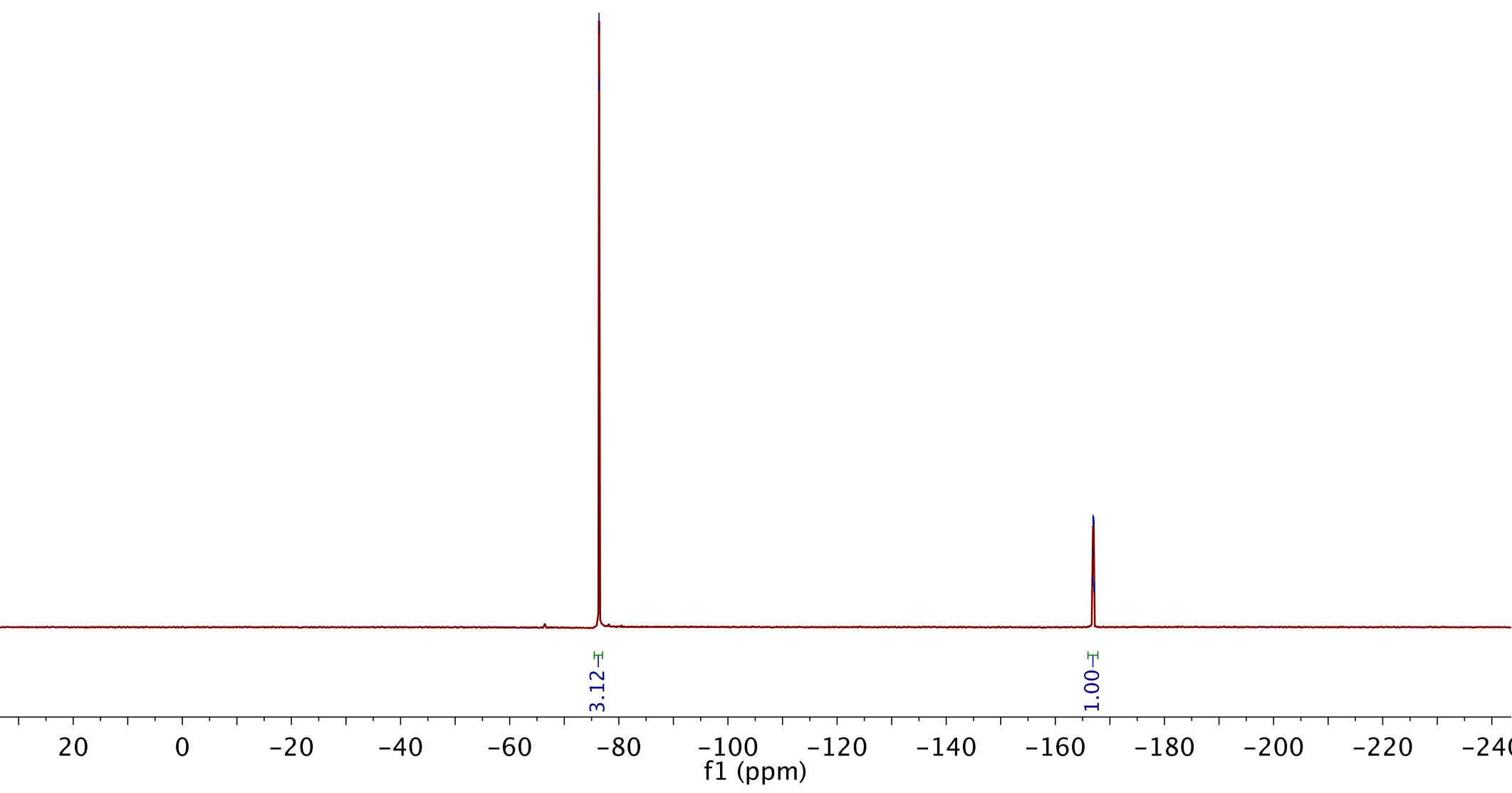


<smiles>COc1ccc(SC(F)(F)c2ccccc2)cc1</smiles>

2aa

${ }^{1} \mathrm{H}$ NMR

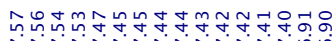

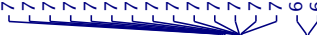

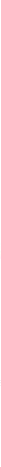


<smiles>COc1ccc(SC(F)(F)c2ccccc2)cc1</smiles>

2aa

${ }^{13} \mathrm{C}$ NMR

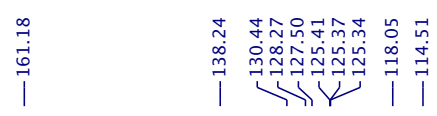

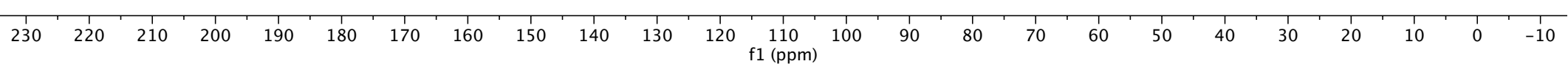


<smiles>COc1ccc(SC(F)(F)c2ccccc2)cc1</smiles>

$2 a a$

${ }^{19} \mathrm{~F}$ NMR

$\stackrel{+}{\stackrel{+}{i}}$

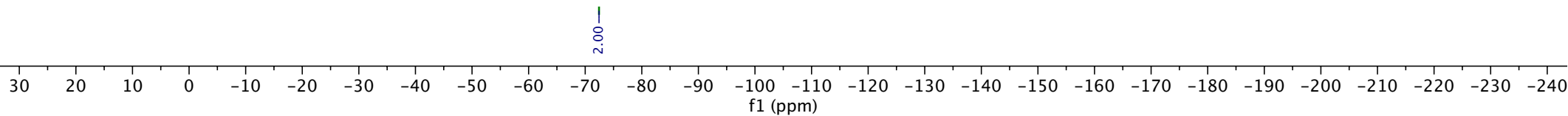




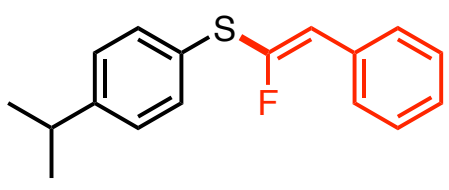

$2 a b$

${ }^{1} \mathrm{H}$ NMR

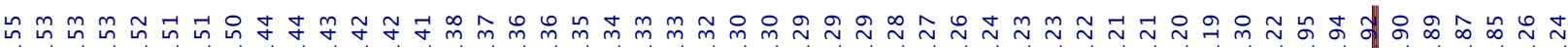

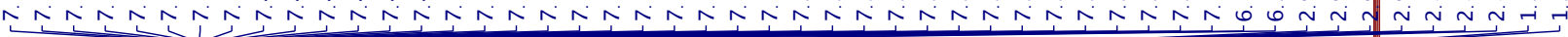

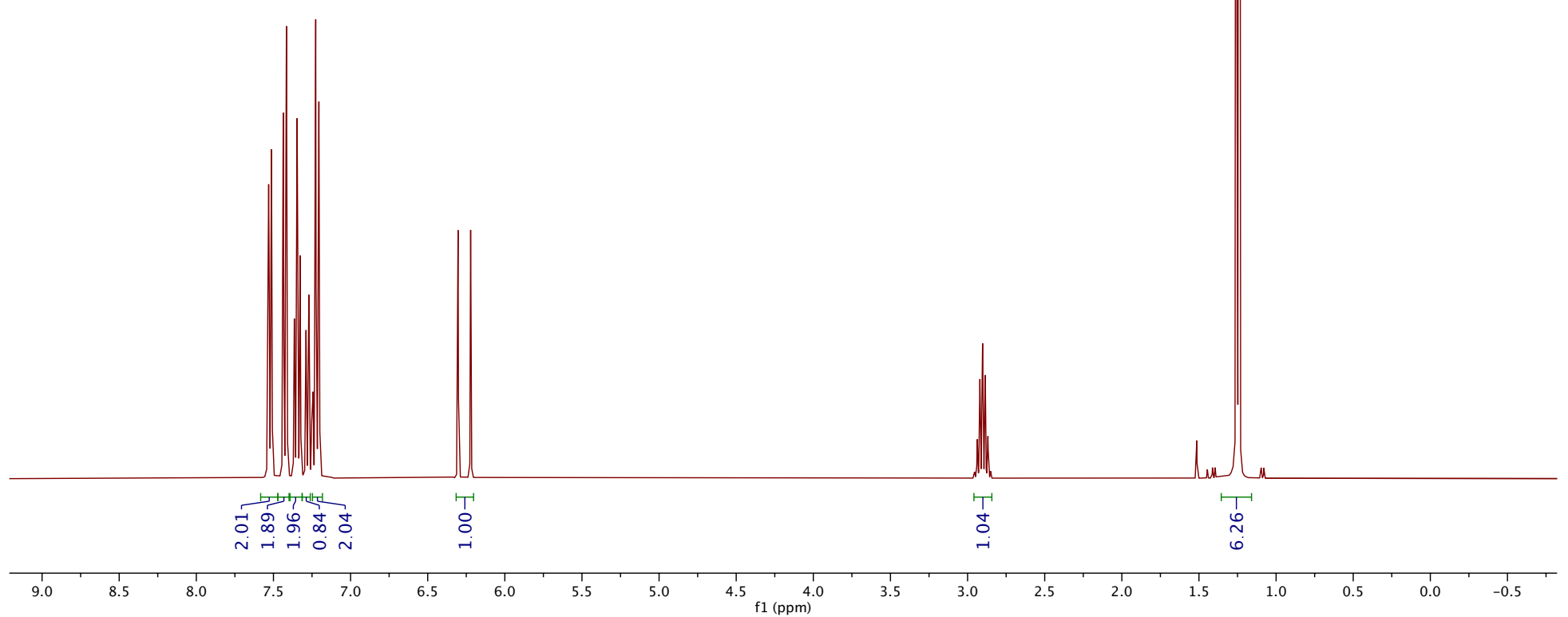




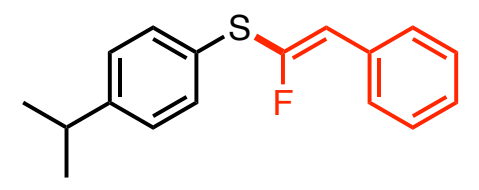

$2 a b$

${ }^{13} \mathrm{C}$ NMR

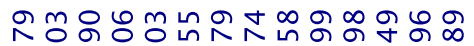

miñ mi்

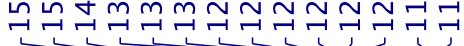

$\infty \quad \hat{\infty}$
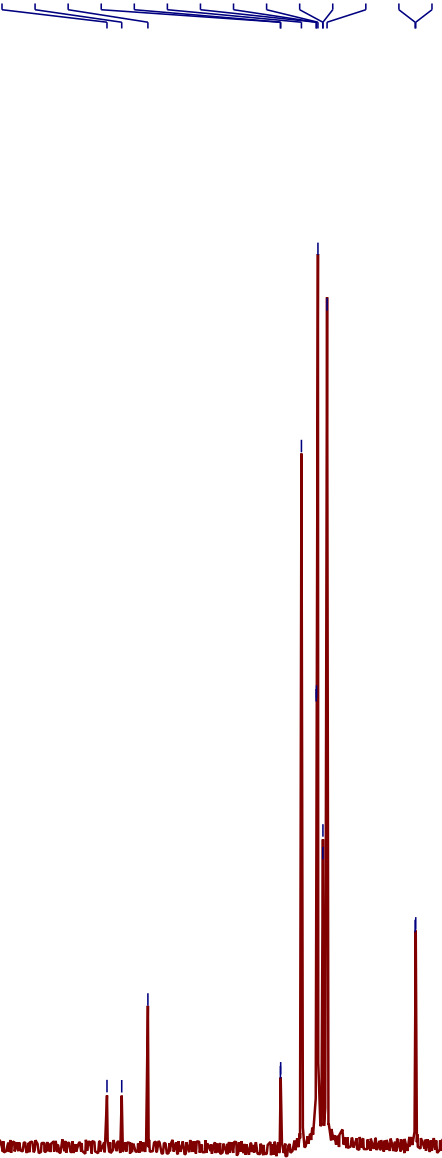

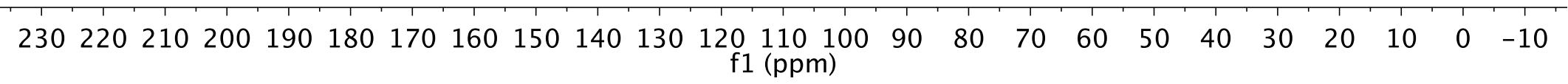




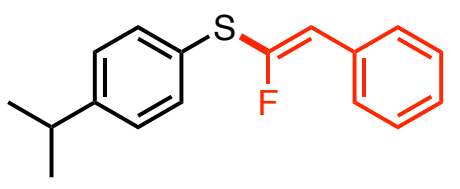

2ab

${ }^{19} \mathrm{~F}$ NMR

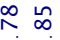

$\infty$

I 